https://theses.gla.ac.uk/

Theses Digitisation:

https://www.gla.ac.uk/myglasgow/research/enlighten/theses/digitisation/

This is a digitised version of the original print thesis.

Copyright and moral rights for this work are retained by the author

A copy can be downloaded for personal non-commercial research or study, without prior permission or charge

This work cannot be reproduced or quoted extensively from without first obtaining permission in writing from the author

The content must not be changed in any way or sold commercially in any format or medium without the formal permission of the author

When referring to this work, full bibliographic details including the author, title, awarding institution and date of the thesis must be given

Enlighten: Theses

https://theses.gla.ac.uk/

research-enlighten@glasgow.ac.uk 


\title{
Constructing and Refining Modules in a Type Theory
}

\author{
Shahad N. Ahmed
}

A thesis submitted for the degree of Doctor of Philosophy to the

Department of Computing Science,

University of Glasgow.

June 1994

(C) Shahad N. Ahmed 1994 
ProQuest Number: 10992296

All rights reserved

INFORMATION TO ALL USERS

The quality of this reproduction is dependent upon the quality of the copy submitted.

In the unlikely event that the author did not send a complete manuscript and there are missing pages, these will be noted. Also, if material had to be removed, a note will indicate the deletion.

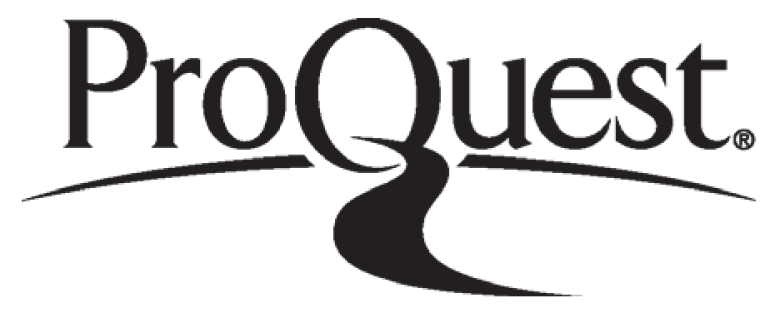

ProQuest 10992296

Published by ProQuest LLC (2018). Copyright of the Dissertation is held by the Author.

All rights reserved.

This work is protected against unauthorized copying under Title 17, United States Code Microform Edition @ ProQuest LLC.

ProQuest LLC.

789 East Eisenhower Parkway

P.O. Box 1346

Ann Arbor, Ml $48106-1346$ 
Theis

10130

Copyl

GLASGOW

UNIVERSITY

LIBRARY 


\begin{abstract}
The need to apply formal specification and development of programs to large problems has highlighted a need for methods to support modular development. This has two aspects: the modular construction of specifications, and the implementation of modular specifications. This thesis is concerned with both these activities.

The main body of work in the development of modular specifications has been carried out in the context of algebraic specification languages, and model-based languages such as Z. However, these languages fail to provide some important mechanisms for structuring specifications. Furthermore, the complex semantics of these languages lead to complicated definitions of what it means for a program to be an implementation of a specification.
\end{abstract}

In this thesis, we show that Martin-Löf's Type Theory provides a framework for both the specification and implementation of program modules; and this framework addresses the shortcomings, noted above, in other specification formalisms. The basic theoretical notion underlying our approach is that a specification is a type, and that an implementation of such a specification is any element in the type.

We present a module specification language, and its associated implementation language. The semantics of both the specification and implementation languages are defined in Martin-Löf's Type Theory. We define some specification building operators for our specification language, and show how modular specifications may be incrementally constructed using the specification operators. We give some laws about the specification operators and show how these laws can be used to reason about, and restructure, modular specifications.

We define a notion of refinement that supports the implementation of modular specifications by systematic mathematical transformation. We give some refinement laws for refining modular specifications. We also define some operators on program modules, and show how these operators can be used to systematically implement modular specifications. 


\section{Acknowledgements}

I am greatly indebted to Joseph Morris, my supervisor, whose encouragement, technical assistance and guidance have been invaluable throughout my studentship. I particularly want to thank Joe for commenting on earlier drafts of this thesis.

The Computing Science Department at Glasgow has provided an excellent environment in which to study and research. I would particularly like to thank Kieran Clenaghan, and Alastair Reid for may interesting discussions during my time in the department.

This work was funded by a Research Studentship from the Science and Engineering Research Council of Great Britain.

Finally, I owe a great deal to Omer, to my parents, and my friends for all their help, encouragement and support. 


\section{Declaration}

The material presented in this thesis is entirely the result of my own independent research carried out at the Department of Computing Science, University of Glasgow under the supervision of Dr. Joseph M. Morris. Any published or unpublished material used by me has been given full acknowledgement in the text. 


\section{Contents}

1 Introduction 1

1.1 Types as Specifications . . . . . . . . . . . . . 3

1.1.1 Propositions as Types .................. 4

1.1.2 Specification and Development........... 5

1.1.3 Different Formulations of Type Theory . . . . . . . . . . 6

1.1.4 Why Martin-Löf's Type Theory? . . . . . . . . . . . . . . 7

1.2 Specifications and Modules . . . . . . . . . . . . 8

1.2.1 Specifications .................... 8

1.2 .2 Modules . . . . . . . . . . . . . . . . 10

1.2.3 Domains: Name-spaces in Specifications and Modules . . . . . 11

1.3 Structured Specifications . . . . . . . . . . . . . . . 11

1.4 Refinement ........................ 13

1.5 Implementation . . . . . . . . . . . . . . . . . 15

1.6 Parameterisation .......................... 15

1.7 Related Work on Specifying Modules . . . . . . . . . . . 16

1.7.1 Type Theoretic Module Specifications . . . . . . . . . . . 16

1.7 .2 Algebraic Specifications . . . . . . . . . . . . . 17

1.7.3 Model-Based Specifications . . . . . . . . . . . . . 18

1.8 This Thesis . . . . . . . . . . . . . . . . . . 19 
2.1 The Type Theory . . . . . . . . . . . . . . . . 21

2.1.1 A Note About Notation . . . . . . . . . . . 21

2.1.2 Judgement Notation . . . . . . . . . . . . . . 22

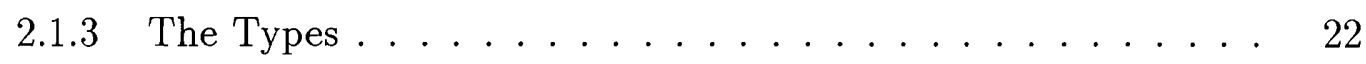

2.1.4 Type Universes ...................... 25

2.2 Extensions to Type Theory . . . . . . . . . . . . 26

2.2.1 Characters and Strings . . . . . . . . . . . 26

2.2 .2 Set Types ......................... 27

2.2.3 Singleton Types . . . . . . . . . . . . . . . 28

2.3 Proof Style . . . . . . . . . . . . . . . . 28

3 Typeful Specifications $\quad 30$

3.1 Introduction . . . . . . . . . . . . . . 30

3.2 Local components . . . . . . . . . . . . . . . . 31

3.2.1 An example of local components--The Mean Module . . . . . 31

3.3 Model-Oriented Specifications . . . . . . . . . . . . . 33

3.3.1 A model-oriented axiomatic specification . . . . . . . . 33

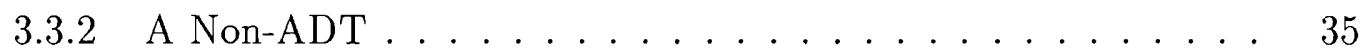

3.4 Nested Specifications and Modules . . . . . . . . . . . . 36

3.4.1 A Nested Specification . . . . . . . . . . . 37

3.4.2 A Nested Module . . . . . . . . . . . . . 38

3.5 Specifications as Record Types. . . . . . . . . . . . . 39

3.6 Parameterisation ......................... 41

3.6.1 Generic Specifications . . . . . . . . . . . . 41

3.6.2 Parameterisation for Structuring . . . . . . . . . . . 42

3.7 Overloading . . . . . . . . . . . . . . . . 44

3.8 An Observational Equality . . . . . . . . . . . . . 45 


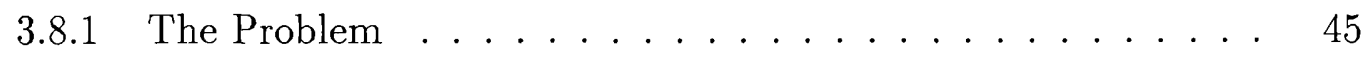

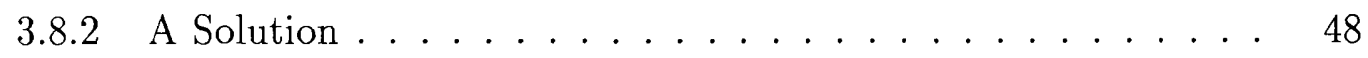

3.8.3 A Generalisation of Observational Equivalence . . . . . . 50

3.9 Conclusion and Summary $\ldots \ldots \ldots \ldots \ldots$

4 The Semantics of Specifications $\quad 52$

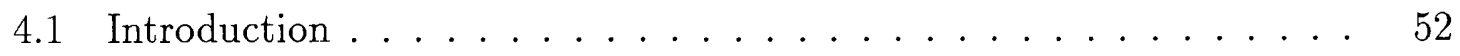

4.2 A review of semantics . . . . . . . . . . . . . . 53

$4.2 .1 \quad$ A running example - Monoids . . . . . . . . . . . 54

4.2 .2 Specifications in the NPS approach . . . . . . . . . 54

4.2 .3 Modules in the NPS approach . . . . . . . . . . 55

4.2.4 Specifications in the deliverables approach $\ldots \ldots \ldots \ldots$

4.2.5 Modules in the deliverables approach . . . . . . . 57



4.3 An Introduction to Our Semantics . . . . . . . . . . . . . 58

4.3 .1 An Example Specification in MLT . . . . . . . . . . 59

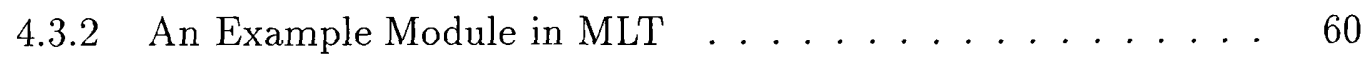

4.4 Signatures in MLT . . . . . . . . . . . . . . 60

4.4 .1 An Illustration $\ldots \ldots \ldots$

4.4 .2 The Signature Judgement _ . . . . . . . . . . . . . 61

4.4 .3 Some Operations on Signatures . . . . . . . . . . . 62

$4.4 .4 \quad$ Scope within Signatures . . . . . . . . . . . . . 63

4.5 Specifications in MLT . . . . . . . . . . . . . . . 64

4.5.1 Some Specification Notation . . . . . . . . . . . . 64

4.6 Computational Elements in MLT . . . . . . . . . . . . 65

4.6.1 An Example Translation . . . . . . . . . . . . 66

4.6.2 Notations for Computational Elements in MLT _. . . . 66

4.6.3 Dependencies within Computational Elements . . . . . . . 67 


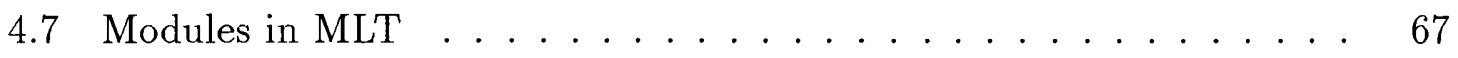

4.7.1 Some Module Notation . . . . . . . . . . . . . . . . . 68

4.8 An Example Translation: PointSpec . . . . . . . . . . . . . . . . . 69

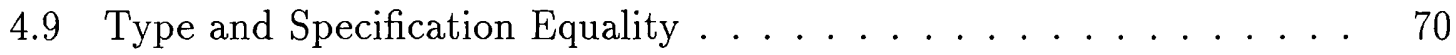

4.9 .1 Weak Type Equality . . . . . . . . . . . . . 70

$4.9 .2 \quad$ Specification Equality $\ldots \ldots \ldots \ldots 71$

4.10 Some Alternative Semantics . . . . . . . . . . . . . . . 72

4.10.1 An Alternative Naming Strategy _ . . . . . . . . . . 72

4.10.2 An Alternative Semantics for Specifications _ . . . . . . 73

4.11 Conclusion and Summary $\ldots \ldots \ldots \ldots \ldots$

5 The Translation Mapping $\quad 75$

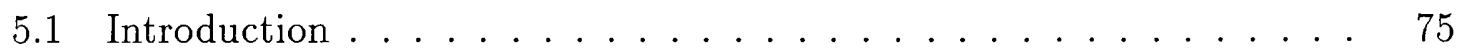

5.2 The Syntax of Specifications and Modules _... . . . . 75

5.3 The Translation Mapping _. . . . . . . . . . . . . 77

5.4 Translating Signatures $\ldots \ldots \ldots \ldots \ldots \ldots \ldots$

5.4.1 Translating Component Identifiers . . . . . . . . . . 78

5.4.2 Translating Loose Signatures . . . . . . . . . . . . . 78

5.4.3 Translating Domains . . . . . . . . . . . . . . . . . 79

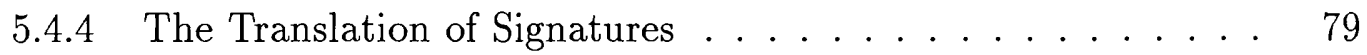

5.4 .5 Type-Checking Signatures . . . . . . . . . . . 80

5.5 Translating Specifications $\ldots \ldots \ldots \ldots \ldots$

5.5 .1 The Open Mapping . . . . . . . . . . . . . . . . . 81

5.5.2 Translating Specifications _. . . . . . . . . . . 81

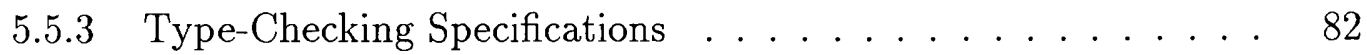

5.6 Translating Computational Elements . . . . . . . . . . . 82

5.6.1 Translating Value Tuples . . . . . . . . . . . . . . . 82

5.6 .2 Translating Domains $\ldots \ldots \ldots \ldots$. . . . . . . 83 
5.6.3 Translating Computational Elements . . . . . . . . . . 83

5.6.4 Type-Checking Computational Elements . . . . . . . . . 84

5.7 Translating Modules ..................... 85

5.7.1 Translating Modules ............... 85

5.7.2 Type-Checking Modules ............... 85

5.8 Conclusion and Summary . . . . . . . . . . . 86

6 Signature and Computational Element Operators $\quad 87$

6.1 Introduction . . . . . . . . . . . . . 87

6.2 Dependent Signatures . . . . . . . . . . . . . . . . 88

6.2.1 The Dependent Signature Judgement . . . . . . . . . . . . 88

6.2 .2 Signature Instantiation . . . . . . . . . . . . . . . . 89

6.3 Signature Renaming . . . . . . . . . . . . . . . 90

6.3.1 A Formal Definition of Signature Renaming . . . . . . . . 91

6.3.2 Laws of Signature Renaming . . . . . . . . . . . . . . . . 92

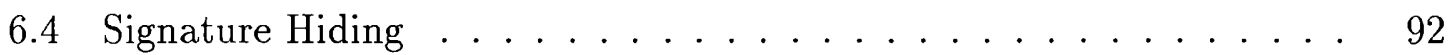

6.4.1 A Formal Definition of Signature Hiding . . . . . . . . . . 94

6.4.2 Laws of Signature Hiding . . . . . . . . . . . . 94

6.5 Signature Concatenation . . . . . . . . . . . . . . 98

6.5.1 A Formal Definition of Signature Concatenation . . . . . . . 98

6.5.2 Laws of Signature Concatenation . . . . . . . . . . 99

6.6 Translating Signature Operators . . . . . . . . . . . . . 102

6.7 Computational Element Renaming . . . . . . . . . . . . . . 102

6.7.1 A Definition of Computational Element Renaming . . . . . . . 103

6.7.2 Laws of Computational Element Renaming . . . . . . . . . . 104

6.8 Computational Element Hiding . . . . . . . . . . . . . 106

6.8.1 A Definition of Computational Element Hiding . . . . . . 106

6.8.2 Laws of Computational Element Hiding . . . . . . . . . . 107 
6.9 Computational Element Concatenation . . . . . . . . . . 108

6.9.1 Definition of Computational Element Concatenation . . . . . . 109

6.9.2 Laws of Computational Element Concatenation . . . . . . . 111

6.9.3 Projection Functions on Computational Elements . . . . . . 113

6.10 Translating Computational Element Operators . . . . . . . . . . 113

6.11 Inverse Operators . . . . . . . . . . . . . . . . . . . . 114

6.11.1 Inverse of Computational Element Renaming . . . . . . . . . 114

6.11 .2 Inverse of Computational Element Hiding . . . . . . . 115

6.12 Discussion and Conclusion . . . . . . . . . . . . . . 116

$\begin{array}{lll}7 & \text { Structured Specifications } & 119\end{array}$

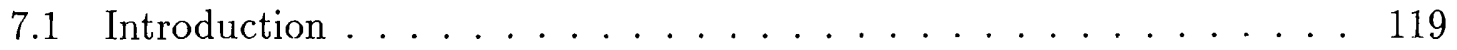

7.2 The Specification Operators _. . . . . . . . . . . . 120

7.3 An Example Structured Specification . . . . . . . . . . . . 123

7.3 .1 A Book Catalogue . . . . . . . . . . . . . . . . 123

7.3.2 A Register of Library Users _. . . . . . . . . . . . . 126

7.3.3 The Book Catalogue Revisited . . . . . . . . . . . . . 129

7.3.4 Discussion of the Example . . . . . . . . . . . . . . 130

7.4 The Semantics of Specification Operators . . . . . . . . . . . . 131

7.4 .1 Rename ...................... . . 131

7.4 .2 Hide . . . . . . . . . . . . . . . . . . . . 132

7.4 .3 Union . . . . . . . . . . . . . . . . . . . . . 132

7.4 .4 Derive . . . . . . . . . . . . . . . 133

7.4.5 Translate . . . . . . . . . . . . . . . 133

7.4 .6 Sum . . . . . . . . . . . . . . . . . 134

7.4 .7 Enrich . . . . . . . . . . . . . . . . . 135

7.4 .8 Summary . . . . . . . . . . . . . . . 135

7.5 Unfolding Specification Operators . . . . . . . . . . 135 
7.6 The Properties of Specification Operators . . . . . . . . . . . 139

7.6.1 Properties of Rename and Hide . . . . . . . . . . . . . . 139

7.6 .2 Properties of Union . . . . . . . . . . . . . . 141

7.6.3 Properties of Sum and Enrich . . . . . . . . . . . . . . . 142

7.6.4 Properties of Derive and Translate . . . . . . . . . . 145

7.7 Discussion and Conclusion . . . . . . . . . . . . . . 146

8 Refinement $\quad 148$

8.1 Introduction . . . . . . . . . . . . . . . . . 148

8.2 Refinement of Types . . . . . . . . . . . . . . . . 149

8.3 Refinement of Specifications . . . . . . . . . . . . 150

8.3.1 An Example of a Specification Refinement . . . . . . . 153

8.4 Basic Properties of Refinement . . . . . . . . . . . . 154

8.5 Refinement of Canonical Specifications . . . . . . . . . 157

8.5.1 Strengthening Restrictions . . . . . . . . . . . . . 157

8.5.2 Refinement by Adding Components . . . . . . . . . . . . 157

8.5.3 Refining Signatures . . . . . . . . . . . . . 158

8.5.4 Refinement by Structuring Canonical Specifications . . . . . . 159

8.6 Refinement of Structured Specifications . . . . . . . . . . . 160

8.6.1 Refinement of Rename and Hide . . . . . . . . . . . . . 161

8.6.2 Refinement of Union . . . . . . . . . . . . . . 162

8.6.3 Refinement of Sum and Enrich . . . . . . . . . . . 163

8.6.4 Refinement of Translate and Derive . . . . . . . . . . . 164



8.7 An Example Refinement . . . . . . . . . . . . . 165

8.7.1 The Refinement of Mean ............... 166

8.7.2 Discussion of Example . . . . . . . . . . . . . . . 169

8.8 Discussion and Summary . . . . . . . . . . . . . 171 
9 Implementation $\quad 178$

9.1 Introduction . . . . . . . . . . . . . . . 178

9.2 Implementors . . . . . . . . . . . . . . . . . . . . . 179

9.2.1 The Rename and Hide Implementors . . . . . . . . . . . . 179

9.2 .2 The Sum Implementor . . . . . . . . . . . . . . . . . . 180

9.2.3 The Derive Implementor . . . . . . . . . . . . . . . . 183

9.3 Some Implementation Laws _. . . . . . . . . . . . . . . . . . . 184

9.3.1 Implementing Enrich . . . . . . . . . . . . . . . 184

9.3.2 Implementing Union . . . . . . . . . . . . . . . 186

9.3.3 Implementing Translate . . . . . . . . . . . . . . . . 188

9.4 An Example Implementation . . . . . . . . . . . . . . . . . . . . . . . 189

9.4.1 The Implementation of $\mathrm{Mean}_{3} \ldots \ldots \ldots$. . . . . . . . . . 189

9.4.2 A Discussion of the Example. . . . . . . . . . . . . 190

9.5 Discussion and Summary . . . . . . . . . . . . . . . . . 191

10 Conclusions 193

10.1 Summary . . . . . . . . . . . . . . . . . 193

10.1.1 Typeful Specification . . . . . . . . . . . . . 193

10.1.2 The Semantics of Specifications . . . . . . . . . . . 194

10.1.3 Structured Specifications . . . . . . . . . . . 195

10.1 .4 Refinement . . . . . . . . . . . . . 196

10.1.5 Implementation . . . . . . . . . . . . . . 196

10.2 A Critical Review of MLT . . . . . . . . . . . . . . . . 197

10.2.1 Specification and Programming in MLT . . . . . . . . . . 197

10.2.2 A Critical Appraisal of Our Approach . . . . . . . . . . 200

10.3 Specification in ECC and MLT: a comparison . . . . . . . . . . . 202

10.4 A Final Comment. . . . . . . . . . . . . . . 206 


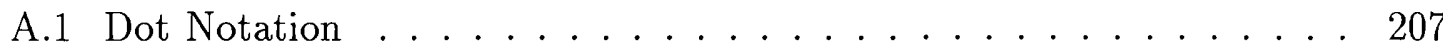

A.1.1 Selector Functions . . . . . . . . . . . . 207

A.1.2 Dot Notation on Computational Elements . . . . . . . . . 210

A.1.3 Dot Notation on Modules and Specifications . . . . . . . . 212

Bibliography

213 


\section{Chapter 1}

\section{Introduction}

As the size and complexity of software systems has increased, so there has been a growing need for better techniques to construct such systems. Some key concepts put forward to control the complexity of software systems are abstraction by specification, modularisation, and stepwise development of programs from specifications. Specifications describe "what" the system must do without necessarily saying "how" it is to be done. Modularisation helps break up large problems into smaller more manageable pieces. Stepwise development helps to bridge the gap between an abstract specification and a concrete implementation in a number of more manageable steps. This thesis is concerned with the specification and development of modular specifications within the single framework of type theory.

Formal specification languages are mathematical notations that allow software systems to be specified in a precise way, so that questions about the design of a system can be discussed and answered before any program code needs to be written. In particular, we can apply mathematical reasoning to the specification of a system to help identify possible problems in its design so that they can be corrected before the system is implemented.

As well as specifying a software system, a formal specification can also play a direct role in making implementations. If we can give a formal relationship between specifications and implementations then it is possible, at least in principle, to derive programs from specifications by correctness preserving mathematical transformations which are usually called refinements. Programs can be developed from specifications by working gradually through a series of refinements until we arrive at a program. This is essentially the discipline of stepwise refinement originally advocated by Wirth [57] and Dijkstra [11].

Specifications of large systems can be as complicated in their structure as programs. We take the view that such specifications should be built in a modular fashion from 
small and relatively independent specification units or modules. Apart from the obvious benefit of breaking large specifications into more manageable pieces, there are other benefits from modularisation. Firstly, it makes the specification more readable and understandable. Secondly, modularisation helps to isolate individual parts of the specification from changes to other parts of the specification; this helps to minimise the amount of re-specification required when we change part of a specification. Thirdly, it may help in making implementations from specifications. Fourthly, it makes it more likely that parts of the specification can be reused in other applications. The last point encourages the building of libraries of reusable specifications and their implementations; this may help reduce the cost - in time and manpower - of specifying and implementing future systems.

The main body of work on modular specifications has been carried out in the context of algebraic specification languages such as CLEAR [5], OBJ [14] and Extended ML [50]. Work has also been done on providing module constructs for model-oriented specification languages; for example, there have been proposals for module facilities for Z [47] and VDM [32]. In our view, the problem with many of these approaches, and in particular the algebraic approach, is that the semantics of such languages are complex and require the complex combination of many different formalisms including logic, set theory and lambda calculus.

Through the work of Martin-Löf $[30,31,44]$ and others, it is known that type theories can also be used to provide a framework for specification and program development. In contrast to algebraic semantics, type theory has the advantage that it unifies first order logic, set theory and lambda calculus within one framework. In particular, the notion of propositions as types leads to a very simple notion of specification and implementation: specifications are types, and the objects in the types are implementations (programs) satisfying the specification. Note the distinction between specifications and implementations.

However, very little work has been done on the modular development of specifications and implementations in type theory, work by Luo [27], Burstall et al [6] and Nordström et al [44] being the exceptions. It is clear to us that many of the features of type theory provide a framework for the modular development of specifications and programs. In particular, the notion of modules as a collection of inter-dependent components can be readily specified in type theory using the notion of dependent types. The use of type theory provides a simple notion of implementation and also provides a calculus that facilitates the implementation of program modules from specifications.

Our thesis is that type theory provides a framework for both the specification and development of program modules. We show how type theory can be used to give a semantics to a module specification language and an associated implementation language. We show how modular specifications may be incrementally constructed by 
combining smaller specifications using specification building operators. We also show how implementations of such modular specifications may be calculated by systematic refinements.

\section{$1.1 \quad$ Types as Specifications}

Whenever we see an expression like $x: P$ in a programming language we often read it as saying " $x$ has type $P$ ". A more unusual way of reading $x: P$ is to think of it as the requirement that says "find a value $x$ that has type $P$ ": we think of type $P$ as a specification, and any value in the type $P$ is acceptable as a correct implementation of $P$. For example, $f: \mathbb{N} \rightarrow \mathbb{N}$ says let $f$ be any function of type $\mathbb{N} \rightarrow \mathbb{N}$, so that both $f=\lambda x . x+1$ and $f=\lambda x . x * x$, to take two examples, are acceptable implementations for $f$.

However, simple types such as we know them from programming languages are of little use as specifications since we cannot express the behaviour of objects such as functions, purely using such types. Our approach is to use a very rich type system, such as Martin-Löf's type theory (MLT), which allows us to specify the detailed behaviour of objects purely by their type; objects include functions and - the main interest to us - modules. The task of implementation is then that of finding any value in this type.

Martin-Löf's type theory has the usual standard types such as $\mathbb{N}$ and $\mathbb{B}$, together with the usual operations on them such as,+- , etc and $\wedge$, $\neg$, etc, respectively. The type theory also includes type constructors such as $\rightarrow$ and $\times$ for forming function and pair types, respectively. Pair values have the usual projection functions fst and snd to extract the individual elements. One of the interesting features of the type theory is that functions and types can be treated as values. For example, both functions and types may be actual parameters to functions.

Another important feature of the type theory is that types themselves have types. In type theory, types of types are called kinds. The kind $U_{1}$ (known as the 1 st universe) is the kind of simple types such as those introduced so far (but $U_{1}$ is not an element of itself); for example, $\mathbb{N} \in U_{1}, \mathbb{N} \rightarrow(\mathbb{N} \rightarrow \mathbb{B}) \in U_{1}$ etc. There is a hierarchy of universes $U_{1}, U_{2}, \ldots$ etc such that $U_{i}(i>0)$ and all its elements are elements of $U_{i+1}$. One unusual type available in the type theory is the empty type $\Phi$ that contains no values. Any specification equivalent to $\Phi$ is treated as an inconsistent specification since to find a value $x: \Phi$ is impossible.

One of the interesting features of Martin-Löf's type theory is the notion of dependent types. One example of such types are the dependent function types. The elements of a dependent function type are functions for which the type of their result is determined 
by the values of the arguments applied to them. The type $\prod x \in P . Q(x)$ is an example of a dependent function type, where $P$ is a type and $Q(x)$ is a type expression defined in terms of variable $x$ of type $P$. The elements of $\Pi x \in P . Q(x)$ are functions that take an element- $p$, say - of type $P$ and return an element of type $Q(p)$. For example, the elements of type

$$
\prod x \in \mathbb{N} \text {. (if } x \neq 0 \text { then } \mathbb{N} \text { else } \mathbb{S} \text { ) }
$$

are functions that take a value $-n$, say-of type $\mathbb{N}$ and if $n \neq 0$ they return a natural, otherwise if $n=0$ they return a string.

Another form of dependent type are the dependent product types. The type $\sum x \in$ $P . Q(x)$ is a typical dependent product type, where $P$ is a type and $Q(x)$ is a type expression defined in terms of variable $x$ of type $P$. The elements of this type are pairs $\langle p, q\rangle$ where $p$ has type $P$ and $q$ has type $Q(p)$; observe that the type of $q$ is dependent on the value $p$. As an example, the elements of type $\sum x \in U_{1} . x$ are all the pairs where the first component is a type and the second component is an element of that type. The pairs $\langle\mathbb{N}, 6\rangle,\langle\mathbb{N}, 0\rangle,\langle\mathbb{B}$, true $\rangle,\langle\mathbb{S}$, "hello" $\rangle$ etc are all elements of $\sum x \in U_{1} . x$.

We note in passing that the normal function types $P \rightarrow Q$ are a special case of the dependent function types and are equivalent to $\prod x \in P . Q$ where $Q$ is not dependent on $x$. Similarly, types of the form $P \times Q$ are equivalent to $\sum x \in P$. $Q$ where $Q$ is not dependent on $x$.

\subsubsection{Propositions as Types}

One of the features of type theory that makes it attractive for program specification is that propositions can be expressed in the type theory we have just been describing. There is no need for additional logical constructs. Martin-Löf's type theory embodies an intuitionistic or constructive logic that is based on identifying propositions with types. If $P$ is a proposition (i.e. a well-formed formula constructed from the logical connectives $\wedge, \vee$ etc), then the "judgement" $p \in P$ means that $p$ is a proof of $P$. In other words, $P$ is identified with the type (or set) of its proofs. That a propositions is true then means that its corresponding type is non-empty (i.e. "inhabited"). The identity between propositions and types is generally attributed to Curry and Howard [20], and is called the principle of propositions as types.

Each of the familiar logical constructs has its counterpart in type theory; for example, $P \wedge Q$ corresponds to the type $P \times Q$, and $\exists x \in P . Q(x)$ corresponds to $\sum x \in P . Q(x)$. Figure 1.1 give the correspondence between the operators of first order logic and their corresponding type constructors in Martin-Löf's type theory. In the first column in Figure 1.1, $P$ and $Q$ denote propositions and in the second column $P$ and $Q$ denote types; $p_{1}$ and $p_{2}$ denote values of type $P$. 


\begin{tabular}{|l|l|l|}
\hline Proposition & Type & Type Name \\
\hline True & $T$ & Top - The unit type \\
False & $\phi$ & Empty - the type with no values \\
$P \wedge Q$ & $P \times Q$ & Cartesian product \\
$P \vee Q$ & $P+Q$ & Disjoint sum \\
$P \Rightarrow Q$ & $P \rightarrow Q$ & Function space \\
$\neg P$ & $P \rightarrow \Phi$ & \\
$\forall x \in P . Q(x)$ & $\prod x \in P . Q(x)$ & Dependent function space \\
$\exists x \in P . Q(x)$ & $\sum x \in P . Q(x)$ & Dependent product \\
$p_{1}=p_{2}$ & {$\left[p_{1}=p_{P}\right]$} & Equality type on $P$ - inhabited iff $p_{1}=p_{2}$ \\
\hline
\end{tabular}

Figure 1.1: Identity between propositions and their corresponding type

Figure 1.1 introduces some types not seen before. The unit type, $T$, contains the single value $t t ; T$ corresponds to the classical proposition True. The equality type $\left[p_{1}={ }_{P} p_{2}\right]$ compares the values $p_{1}$ and $p_{2}$ of type $P$ for equality. If $p_{1}$ and $p_{2}$ are equal then the type $\left[p_{1}={ }_{P} p_{2}\right]$ is inhabited by the constant eq. If $p_{1}$ and $p_{2}$ are not equal then $\left[p_{1}={ }_{P} p_{2}\right]$ is equivalent to the empty type.

The propositional connectives $(\Lambda, \vee$ etc $)$ are used to stand for the corresponding type connectives $(\times,+$ etc) whenever types are used as propositions; for example, if the type $P \times Q$ is used as a proposition we shall write it as $P \wedge Q$. The precedence for each type connective is equivalent to the precedence of the logical connective it identifies, and grouping the logical connectives in decreasing precedence we get: $=; \neg ; \wedge, \vee, \Rightarrow$.

Of course one has to give an argument that the correspondence between propositions and types is a reasonable one, and that argument has been given, e.g. in [30, 31, 44]. The actual values in a propositional type are not important, its truth is determined only by whether the type is inhabited. Any proposition true intuitionistically is true classically, but one limitation of constructive logic is that propositions that can be proved true classically cannot always be proved true constructively.

\subsubsection{Specification and Development}

Martin-Löf [30] and others [44] have shown that it is possible to express the properties of programs by combining the notion of dependent types and propositions as types. For example, the following type specifies a function for computing the natural number square root:

$$
\sum f \in \mathbb{N} \rightarrow \mathbb{N} .\left(\forall n \in \mathbb{N} . f(n)^{2} \leq n \wedge(f(n)+1)^{2}>n\right)
$$

The "for all" proposition in the above type stands for the corresponding $\Pi$-type under the principle of propositions as types. The proposition holds for all $f$ integer square root functions. Since the above type is a $\sum$-type, its elements are pairs whose first 
component contains a natural number square root function; the second component is a value of the propositional type that constrains $f$. The actual value of the second component-which is called a witness - is not important, it simply witnesses that the first component satisfies the proposition defined by the above type.

Developing a program from a specification is the activity of constructing an element of the specification regarded as a type. The language used to express the values in the types available in MLT constitutes a functional programming language. So programs are expressions without assignment and other side-effects. The development of programs from specifications is formalised in a constructive programming logic which has inference rules for both deducing the correctness of a program, and constructing programs to meet specifications. The constructive implementation of small specifications in type theory is reasonably developed in the literature [3, 44], but little work has been done on the constructive implementation of large specifications.

\subsubsection{Different Formulations of Type Theory}

\section{Martin-Löf's Type Theory}

There are several different formulations of Martin-Löf's type theory [28, 29, 30, 31]. The formulation of MLT in [29] is intensional. Intensional means that judgemental equality is understood as definitional equality; in other words, two types are equal only if there is a type rule stating that they are equal. One consequence of an intensional theory is that equality is decidable.

The type theory we use is an extension of that presented in [30,31]. This theory is said to be polymorphic and extensional. Extensional equality means the same as in ordinary set theory: two types are equal if they have the same elements. However, judgemental equality is not decidable in the extensional theory. In the extensional theory, any well-typed expression can be computed to a normal form. Furthermore, the extensional theory computes expressions using lazy evaluation.

One of the features of MLT is that it is open to extension, and our thesis makes use of several extensions to the theory. Nordstrom et al [44] and Constable et al [8] have extended the theory to include list and subset types - the work of Constable et al is done in the context of constructing the Nuprl proof development system. Backhouse et al [3] show how the theory can be extended in a uniform manner to introduce a rich collection of data types such as sets, bags, guarded expressions and polymorphic functions. 


\section{The Calculus of Constructions}

The Calculus of Constructions (CC) [10] is another type theory used for program specification and development. CC differs from MLT in several ways. Firstly, it is impredicative: there is no hierarchy of type universes, although there is a kind Type of all types (but Type $\notin$ Type). Secondly, it does not define a dependent sum type constructor $\left(\sum\right)$ : this omission makes CC less suited to making structured specifications since, as we shall see later, the ability of $\sum$ to combine types and express dependencies within types is important for making module specifications. Thirdly, $\mathrm{CC}$ only introduces a primitive collection of types: data-types, such as lists, must be constructed from these primitive types. Fourthly, CC makes a distinction between types used as data types and types used as propositions: there is a kind Prop of all types used as propositions, and an axiom that states that all elements of Prop are also elements of Type. So propositions are types, but types are not necessarily propositions. The advantage of defining an impredicative universe Prop is that second-order logic can be interpreted in CC.

More recently, Luo $[24,26]$ has extended CC with predicative type universes Type ${ }_{1}$, Type $_{2}, \ldots$ etc and $\sum$-types to produce the Extended Calculus of Constructions (ECC). ECC can be viewed as an extension of MLT since ECC contains the key features of MLT, and also includes an impredicative universe for propositions.

\subsubsection{Why Martin-Löf's Type Theory?}

In principle, there is no reason why the methodology presented in this thesis could not be formulated in other type theories such as Nuprl [8]: any type theory with propositions as types and $\sum$-types could be used. Indeed, during our work we have become aware of the work of Luo [27] in specifying and refining structured specifications in ECC.

Although we use an extensional version of MLT, the work presented in this thesis does not rely on the extensional features of the type theory. Indeed, our work can also be formulated in an intensional version of MLT, such as that presented in [44]. Intensional type theories lend themselves to the implementation of machine based proof development systems since type checking and equality are decidable in such theories. Consequently, formulating our work in an intensional version of MLT would make it easier to implement a proof development system for verifying properties about our specifications and implementations. However, we choose to work in the extensional formulation of Martin-Löf's type theory [30,31, 44], partly since it is amenable to extension, but also because it is well established in the literature. 
In summary, Martin-Löf's type theory provides a single framework for the study of both program specification and development: the formal language of type theory is used as a programming language, a specification language, and a programming logic.

\subsection{Specifications and Modules}

In this thesis, we are concerned with the theory underlying the construction and development of modular specifications. We develop a simple module specification and implementation language whose semantics is given by MLT. The purpose of this section is to introduce the style of module specifications used in this thesis.

By definition, a specification is a type. In particular, a specification consists of the product of a signature, and a proposition over the components defined in the signature. A signature is a type and is similar, syntactically, to a signature in the programming language Standard ML [33], and a tuple type as in Quest [7]. The proposition-which is also a type-specifies the properties that an implementation of the specification must satisfy; the proposition is called the restriction of the specification.

The members of a specification are implementations that satisfy the specification and are called modules. Modules are analogous to ML structures and Quest tuples, where each component has a name, and can be referred to via a Pascal style dot notation. A module that satisfies a specification must meet two conditions: firstly, it must contain all the components defined in the signature of the specification, and secondly, its components must satisfy the restriction of the specification.

\subsubsection{Specifications}

The simplest form of specifications are called canonical specifications. Canonical specifications are the basic building blocks of more complicated structured specifications. The specification Catalogue, given in Figure 1.2, is an example of a canonical specification that specifies a catalogue of books for a library. The declarations following the keyword Elements denote the signature of the specification, and the proposition following the keyword Restrictions is the restriction of the specification. The first component Book has type $U_{1}$; so Book is a type. Note that there are no specific constraints on Book: Catalogue is only intended to specify a "container" for Book values and it says nothing about how to make, or modify, Book values. Therefore, Catalogue can be viewed as a partial specification that specifies the book catalogue without worrying about details concerning books at this stage. It is our intention to add details about Book later: in general, we shall advocate a style of specification 


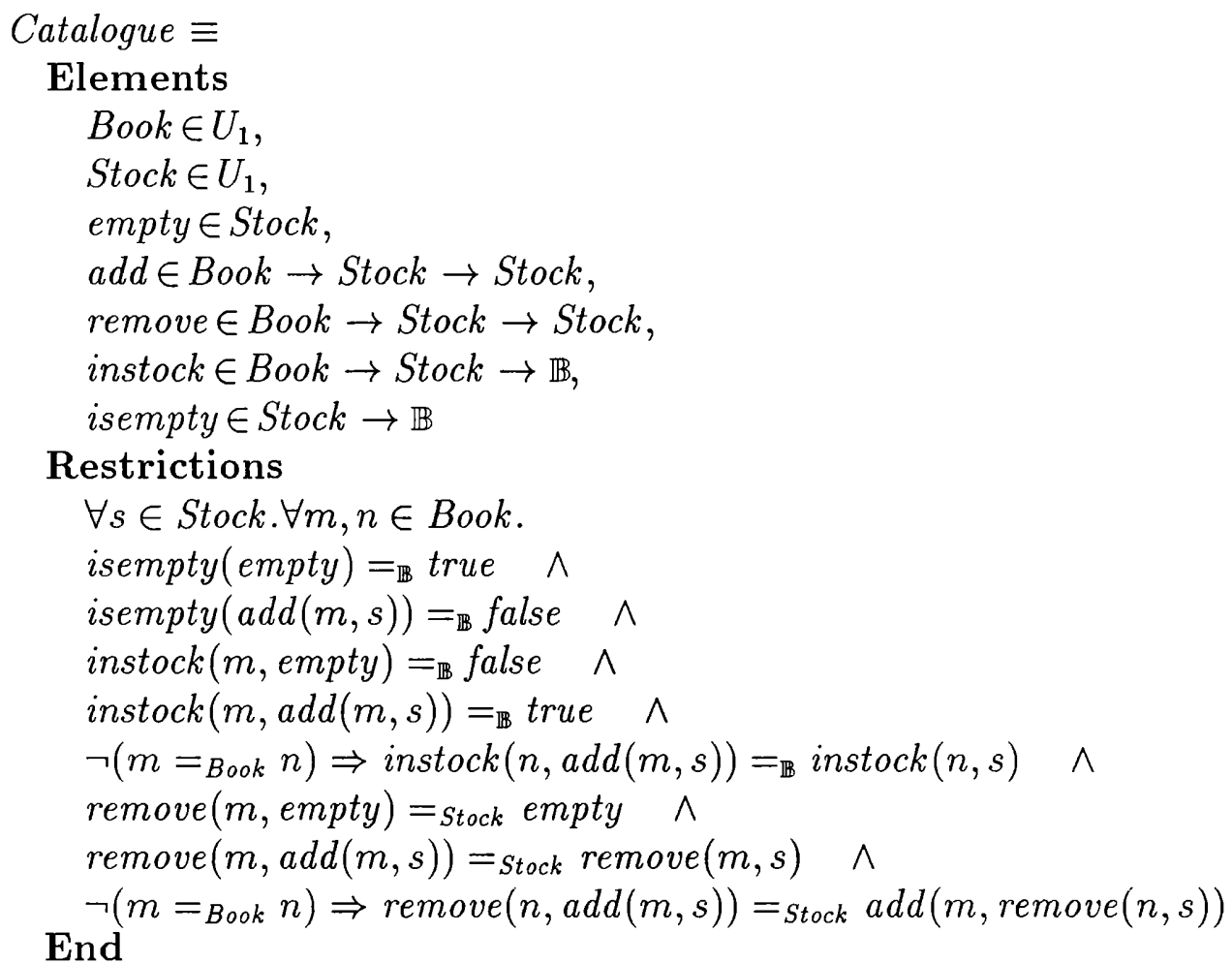

Figure 1.2: The specification of a Book Catalogue

that often begins by making a partial specification and then adding detail to it until we arrive at a final specification. The second component Stock is also specified to be a type. The values of Stock denote collections of books. The signature of Catalogue also includes operations to add and remove books to and from a library stock. The operation instock queries whether a book is in stock; empty represents is an empty stock value; and isempty queries whether a stock value is empty.

Note that the types of the operations in Catalogue are dependent on the type components Book and Stock. In general, the type of a component may be dependent on any component preceding it in the signature; this includes non-type components such as functions, although in practice, we rarely specify dependencies on non-type components. The restriction may be any proposition allowed in type theory. In practice, the restriction usually takes the from exemplified by the restriction of Catalogue; a conjunction of equations that is quantified over the types defined in the signature. Some of the equations in the restriction of Catalogue are guarded using logical implication; such equations are called "conditional equations".

The semantics of specifications-and hence modules-are defined in terms of the dependent sum type $\left(\sum\right)$ : the semantics will be given, in Chapters 4 and 5 , by translating specifications into terms denoting types in Martin-Löf's type theory. The idea that $\sum$-types can be used to give the type of modules was reported independently 


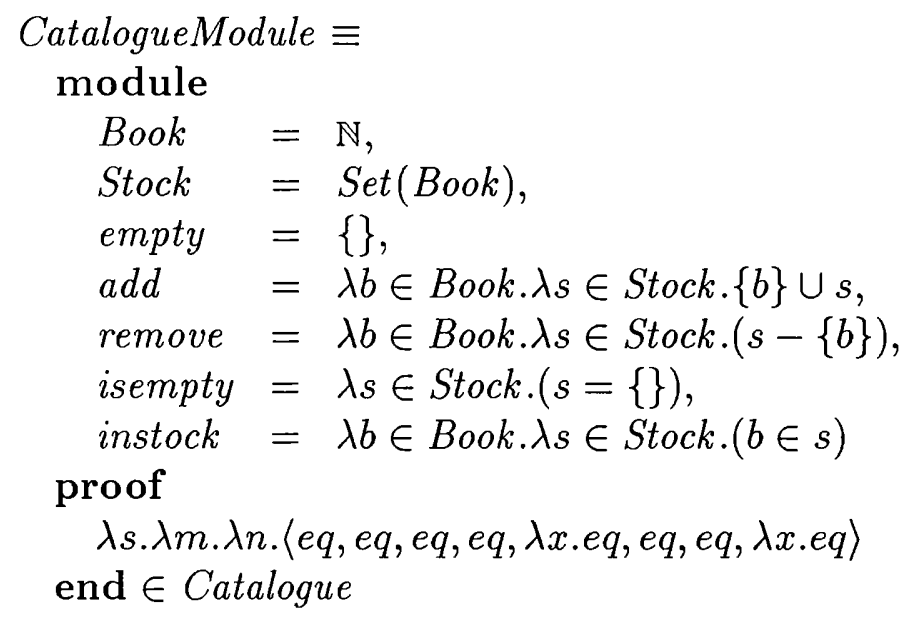

Figure 1.3: A Module Satisfying Catalogue

in [43] and [34], but only [43] shows that the behaviour of the components in a module can be specified by adding propositions to its type. A detailed discussion of [43] is given in Chapter 4.

\subsubsection{Modules}

Figure 1.3 gives an example of a module, named CatalogueModule, that satisfies the specification Catalogue. Note that we have chosen to implement the Book component in CatalogueModule by the type $\mathbb{N}$; so book values are implemented as naturals which are intended to denote individual books. The stock of the library is represented by a set of Book values. We call that part of a module obtained by omitting the keyword proof and the value that follows it, the computational element of the module. The value after the keyword proof may seem a little unusual; it is called a witness and it proves that CatalogueModule satisfies Catalogue. For the moment, we ignore witnesses - they are discussed in Chapter 4-and we will often omit them when writing modules, replacing them by "...".

The components in a module may be referred to via their names using a Pascal style dot notation. For example, the Book component may be referenced by CatalogueModule.Book; so CatalogueModule.Book $=\mathbb{N}$. The following give some more example of dot notation:

CatalogueModule.isempty $=\lambda s \in \operatorname{Set}(\mathbb{N}) \cdot(s=\{\})$

CatalogueModule.isempty (CatalogueModule.empty $)=$ true

When we refer to a component by dot notation, all its dependencies on other components are removed by substitution. For example, CatalogueModule.Stock $=\operatorname{Set}(\mathbb{N})$; the dependency Stock has on Book has been removed by substituting the actual implementation of Book for the name Book. 
The computational element of a module can be used like a record value. The types of computational elements will be defined to be signatures. If a module satisfies a specification then its computational element is a member of the signature of the specification; the witness of the module is a member of the restriction of the specification. We write computational elements like modules but omit the keyword proof and the witness-computational elements should not be confused with modules in which we replace the witness by "...".

\subsubsection{Domains: Name-spaces in Specifications and Modules}

We call the collection of component names used in a specification the domain of the specification, and we call the collection of component names used in a module the domain of the module; and so on for signatures and computational elements. As the component names in specifications and modules are given linearly, we treat domains as lists of names.

The issue of component names within specifications and modules is not addressed in any detail in [44] and [34]; component names are treated as nothing more than bound variables. Consequently, in [44] one specification cannot refer to the components of another specification by name. Furthermore, [44] does not name components in modules: components are referred to by their position in a module.

We take the view that component names play a dual role: on the one hand they are used as bound variables to specify the dependencies between components within specifications and modules, and on the other hand they allow a specification, or module, to refer to components within other specifications and modules. We will give names a formal semantics that caters for both theses roles. A formal treatment of names in modules leads to a formal definition of dot notation on modules. As we shall see in the next section, names are also used by specification operators to allow us to identify components to be renamed, hidden or combined.

\subsection{Structured Specifications}

The modular development of specifications is supported by operators that allow us to combine and modify specifications. The specification operators are formally defined as transformations that take a number of arguments, which may be specifications, and return specifications as results. The specification operators include renaming, which is used to change the names of components in a specification; and hiding, which is used to remove components from a specification while still preserving the behaviour of 


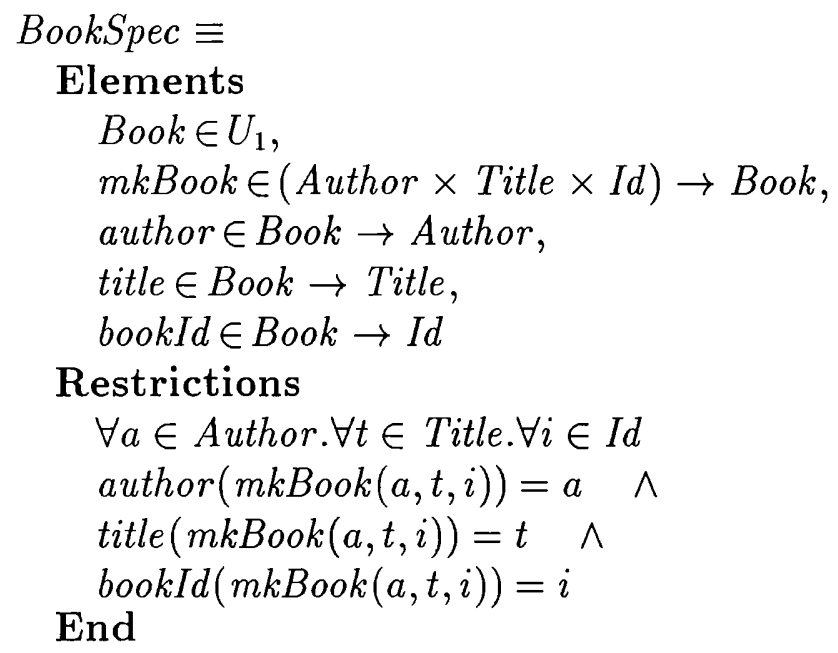

Figure 1.4: A Specification for Books

the remaining components. Other operations include sum, which combines two specifications into one that contains all the components from both; and enrichment which is used to add new components and restrictions to specifications. The operations can be used to construct large specifications incrementally in a piecewise manner by constructing smaller relatively independent specifications which are combined, using the operators, to produce large specifications. The operators also have useful algebraic properties which can be used to reason about structured specifications.

We now give some small examples of incrementally constructing specifications. We consider extending our book catalogue example by adding operations on books. We develop a separate specification called BookSpec-given in Figure 1.4-which specifies a book type whose values give the author, title and book identifier of a book. In practice, we would parameterise BookSpec with respect to the types Author, Title and $I d$, but for simplicity we shall assume these types as being given in some global context; for example, Author and Title could be defined as strings, and $I d$ by N.

Using the sum operator $(+)$, we may construct a new specification for the book catalogue by combining Catalogue with BookSpec to give the specification Catalogue : $_{2}$ :

$$
\text { Catalogue }_{2}=\text { Catalogue }+ \text { BookSpec }
$$

The new specification Catalogue ${ }_{2}$ specifies modules that contain all the components specified by Catalogue and BookSpec, such that these modules satisfy the original constraints on both Catalogue and BookSpec. There is a potential problem with nameclashes when summing Catalogue and BookSpec since both contain a component, $B o o k$, with the same name. The sum operator handles such name-clashes by treating each occurrence of Book as being distinct, so that Book appears twice in Catalogue C $_{2}$ we say more about resolving name-clashes in Chapter 7. The restriction of Catalogue $_{2}$ is the conjunction of the restrictions on Catalogue and BookSpec. 
The hide $(\backslash)$ operator is used to remove components from the signature of a specification. For example, the specification Catalogue 3 illustrates the use of the hide operator to remove the components mkBook, author, title and bookId from the signature of Catalogue $_{2}$ :

$$
\text { Catalogue }_{3}=\text { Catalogue }_{2} \backslash\{m k B o o k, \text { author }, \text { title, bookId }\}
$$

If we were to continue to specify a complete library system, then we might wish to specify a module for a register of library users. Such a specification would be very similar to Catalogue, as we would require operations to add and remove library users to and from a library. We would also require operations to query the status of a register and individuals. One possible specification of a register module may be defined simply by renaming some of the components of Catalogue. The specification Register, below, shows the use of the rename operator to specify a module for a register of library users. The renaming specifies that Book is to be renamed as Person, Stock is to be renamed as Users etc.

$$
\begin{array}{r}
\text { Register }=\text { Catalogue }[\text { Book } \backslash \text { Person, Stock } \backslash \text { Users, add } \backslash \text { addUsers, } \\
\text { remove } \backslash \text { RemoveUsers, instock } \backslash \text { registered }]
\end{array}
$$

In practice, when we reuse a specification, such as Catalogue, it may contain components that we don't require, and these may be hidden using the hide (or derive-see later) operator. Additionally, we may want to add some new components specified in terms of existing components, and these may be added using the enrich operator $(\triangleleft)$. For example, suppose we require an extra operation within a register module so that we can calculate the number of registered users. The new operation, which we call size, may be specified as the following enrichment of Register:

$$
\begin{aligned}
& \text { Register }_{2}=\text { Register } \triangleleft \Lambda \text { Elements } \\
& \text { size } \in \text { Users } \rightarrow \mathbb{N} \\
& \text { Restrictions } \\
& \forall s \in \text { Users. } \forall m \in \text { Person. } \\
& \text { size }(\text { empty })=0 \wedge \\
& \operatorname{size}(\operatorname{addUser}(m, s))=\text { if registered }(m, s) \text { then } \\
& \text { size }(s) \\
& \text { else } \\
& \operatorname{size}(s)+1
\end{aligned}
$$

End

\subsection{Refinement}

We will define a notion of refinement between specifications which facilitates the formal development of program modules from specifications. Refining a specification may be regarded as the task of adding implementation decisions about properties of 
the specification that were left open by the specifier-the choice of algorithms, data structures and error messages for example. In the context of type theory, we regard refinement as being different from implementation. Specifications are types, and any refinement of a specification is also a specification and hence a type. Implementations are values of a type, and so we regard the implementation task as that of finding values within a type. We don't make implementations in one go, instead we proceed to an implementation by refining the specification. Implementation becomes easier the more we refine a specification, and so we keep refining a specification until we arrive at a specification that strongly suggests an implementation. Then we proceed to find an implementation from the specification by using a constructive proof.

We give a type-theoretic definition of refinement that allows all types used as specifications - not just module specifications-to be refined. However, we concentrate on the refinement of module specifications. In its simplest form, module refinement says that a specification $S P_{1}$ refines to a specification $S P_{2}$, written $S P_{1} \sqsubseteq S P_{2}$, if every implementation of $S P_{2}$ is an implementation of $S P_{1}$. In other words, $S P_{1} \sqsubseteq S P_{2}$ if $S P_{2}$ is a subtype of $S P_{1}$. The actual definition of module refinement is more powerful than that described above as it will allow data refinements in which the signature of $S P_{2}$ may differ from $S P_{1}$. Typically, specifications are data refined by adding new components to their signature, or by changing the type of components within their signature. When data-refining $S P_{1}$ to $S P_{2}$, the relationship between the signature of $S P_{1}$ and $S P_{2}$ is given by supplying an abstraction function which relates implementations of $S P_{2}$ to implementations of $S P_{1}$.

The module refinement relation is transitive. Transitivity allows us arrive at a refinement via a sequence of intermediate refinements. Moreover, we may refine a structured specification by refining its individual parts in relative isolation; in other words, the specification operators are monotonic with respect to the refinement relation. The practical consequence of monotonicity is that the "shape" of a specification can be carried over to its refinements. Transitivity and monotonicity give the basic requirements necessary for stepwise development of specifications.

In general, verifying that one specification is a refinement of another is difficult. Therefore, we supply a collection of refinement laws that can help make refinements. Module specifications tend to be large and complex, and in order to refine them we need a systematic approach that simplifies the refinement task. We advocate a piecewise approach to refinement which decomposes the refinement of large specifications into the task of refining their constituent pieces in relative isolation; laws are given that support this methodology. Not all specifications are suited to piecewise refinement, and we consider some refinement laws that use the specification operators to decompose specifications into forms more amenable to piecewise refinement. 


\subsection{Implementation}

Once a specification has been sufficiently refined, its refinement can be implemented to produce a program. A specification is usually refined until it is in a form in which the restrictions of each of its constituent canonical specifications suggests an implementation. Each canonical specification can then be implemented by a constructive proof. This step is usually clerical. Consequently, the implementation of canonical specifications is not very interesting and is not considered in this thesis. Instead, we consider the implementation of structured specifications (i.e. specifications that use the structuring operators). We outline how the shape of a specification may sometimes be used to decompose the implementation task into that of implementing individual pieces of a specification, so that the implementations may be glued together to form an implementation of the specification.

The piecewise approach to refinement, described in the previous section, advocates refining modular specifications by refining their individual parts in relative isolation. Ultimately, the individually refined parts must be implemented and combined. For example, suppose we are given two specifications $S P_{1}$ and $S P_{2}$ that are combined using the sum operator to make a new specification $S P_{1}+S P_{2}$. If we can implement $S P_{1}$ by module $m_{1}$, and implement $S P_{2}$ by a module $m_{2}$, then we might ask whether there is a combinator at the implementation level that can combine $m_{1}$ and $m_{2}$ to give an implementation of $S P_{1}+S P_{2}$. We will show that for most of our specification operators, analogous combinators do exist at the implementation level. However, we show that some of the implementation combinators, such as enrich, only guarantee a correct implementation under certain conditions.

\subsection{Parameterisation}

One important way specifications can be structured is by parameterisation. Parameterisation of specifications allows a specifier to make general purpose specifications that may be instantiated in different ways. Parameterisation therefore provides a useful mechanism for the reuse of specifications. The higher-order features of type theory are particularly suited to describing parameterised specifications. Parameterised specifications can be described directly as functions that take a number of arguments, possibly including specifications, and return a specification as result. We show how this style of parameterisation can be used to structure large specifications, and also to decompose large specifications when developing implementations. We can also introduce parameterised modules. Parameterised modules are functions that return program modules as their result. We show that parametrised modules 
can be specified by using the dependent function type constructor ( 1 ). In particular, we show that parameterised specifications can be implemented as parameterised programs, and that $\Pi$-types play a useful intermediary role in the development of parameterised and non-parameterised specifications.

\subsection{Related Work on Specifying Modules}

In this section, we discuss related work on specifying and implementing modules in various formal frameworks. We begin by mentioning two approaches to constructing modules in type theory. Then we consider related work in algebraic and model-based specification methods. Roughly speaking, the aim of a model-based specification is to build an abstract model of the program being specified; this is in contrast to algebraic specifications, which describe a system in terms of its desired properties without constructing an explicit model.

\subsubsection{Type Theoretic Module Specifications}

Nordström and Petersson $[43,44]$ were the first to show that a type theory such as MLT could be used to specify modules, and their work lays down the foundation on which our thesis is based. Nordstrom et al show that abstract data types-such as stacks-can be specified as $\sum$-types whose elements are tuples containing a type together with associated operations on the type; they consider tuples to be modules. They also show that parameterised modules can be defined as functions returning modules, and may be specified by dependent function types ( $\Pi$ ). However, Nordstrom et al do not consider the specification of modules that are not abstract data types; nor do they investigate the use of specification operators, or a definition of refinement for developing implementations. A more detailed review of the semantics of Nordstrom et al is given in Chapter 4 which discusses the semantics of specifications.

Luo [27] shows that modules can be specified, and implemented, in the Extended Calculus of Constructions (ECC). Luo defines specifications not as types, but as pairs; the first component is a type called the structure type and specifies the signature of a module; the second component is a predicate-on the elements of the structure type-specifying the behaviour of the components declared by the structure type. Luo argues that, by using pairs, it is easier to separate the computational content of a specification (expressed by the structure type) from the axiomatic requirements; this, it is argued, helps to avoid computationally redundant witnesses appearing in implementations of such specifications. The idea of defining specifications as pairs is also advocated by Burstall et al [6] who use such pairs to make specifications-which they call "deliverables" - in ECC. 
Luo also defines a refinement relation on specifications which is similar to that described in section 1.4, and it is also similar to the notion of refinement for deliverables, given in [6]. Luo also identifies two general classes of specification operators called constructors and selectors which can be used to define operations such as joining two specifications and enriching a specification: constructors and selectors are also monotone with respect to the refinement relation. Name-space issues within specifications and implementations are not considered, so that operations such as renaming and hiding components are not considered. We give a detailed comparison between our work and Luo's in Section 10.3.

\subsubsection{Algebraic Specifications}

Although we are concerned with the specification and development of modules in type theory, our work is closely related to specifying modules using algebraic specifications. Algebraic specifications provide a means of describing data structures in an abstract property oriented way. Algebraic specifications specify software systems as a collection of abstract data types (ADT's). Superficially, algebraic specifications are similar to the style of module specifications illustrated in section 1.2.1, consisting of a signature and axioms. The signature of an algebraic specification takes the form of a collection of names of types called sorts, together with the names and types of the primitive operations on the sorts. The axioms specify the behaviour of the operations in terms of the relationship between the operations declared in the signature.

One of the first and most influential algebraic specification languages was CLEAR [5]. CLEAR is designed to allow algebraic specifications to be constructed in a structured manner. The CLEAR language has operators such as enrichment, amalgamated sum for combining specifications, and an operator called derive which is used to remove sorts and functions from a specification. CLEAR also allows specifications to be parameterised by other specifications. Most other algebraic specification languages such as OBJ [14], Larch [17], ASL [51, 55] and Extended ML [49, 48] contain the features of CLEAR. Larch is notable as it consists of a core algebraic specification languagecalled the Larch shared language - and a collection of "interface languages" each of which is targeted to programming in a specific programming language. Extended ML is a "wide spectrum" language where the programming language Standard ML is a subset of the specification language. ASL was designed as a kernel language for constructing structured specifications; it includes general purpose specification operators which can be combined to produce further specification operators. Much of the work in this thesis is influenced by the work of Sannella and Tarlecki $[49,48]$ on ASL and Extended ML. 
Semantically, an algebraic specification denotes a class of models. The models are algebras whose carriers model the sorts of a specification, and whose operations model the operations specified in the signature of a specification. There are four main approaches to determining which algebras are models of a specification: initial semantics [15], terminal semantics [54], loose semantics [16], and ultra-loose semantics [56]. Roughly speaking, the initial, terminal and loose models of a specification are algebras whose carriers and operations satisfy the axioms, provided the carriers do not contain "junk" terms; junk terms are values that cannot be constructed using operations supplied by the signature of a specification. In the ultra-loose approach, models may contain junk terms. The type-theoretic semantics we advocate is similar to algebraic semantics in the sense that values in a type can be viewed as models of a specification. The type-theoretic semantics is closest to the ultra-loose semantics since there is no restriction on junk terms.

Programs can be developed from an algebraic specification by refining it towards a specification that is so low level that it can be regarded as a program; unlike type theory, the models of an algebraic specification are not regarded as implementations. There are several definitions of refinement for algebraic specifications $[12,18,51]$. Of particular interest to us is the notions of refinement for ASL developed by Sannella and Wirsing [51]: an ASL specification $S P_{1}$ is refined by $S P_{2}$ iff every loose model of $S P_{2}$ is a loose model of $S P_{1}$. Sannella and Tarlecki [50] have introduced a generalised definition of ASL refinement, called "constructor" implementation, which includes the notion of a refinement map similar to our abstraction function described in section 1.4. The definition of refinement introduced in Chapter 8 is a type-theoretic equivalent of constructor implementation.

There are some important differences between the algebraic and type theoretic approach to specifying modules. For example, when specifying ADT's in type theory we may use one of the built in types of MLT as a model for a sort; this allows axioms to be specified in terms of a particular model, rather than in an abstract property oriented style. Furthermore, since specifications are types, we can use specifications in the same way we use data-types such as $\mathbb{N}, \mathbb{B}$ etc. For example, we can specify a module to be a component within another module; and modules can be the arguments and results of functions. These and other advantages of using types to specify modules are discussed in Chapter 3.

\subsubsection{Model-Based Specifications}

Another important class of specification languages are the model-based specification languages such as Z [52], The Vienna Development Method (VDM) [21] and the refinement calculus $[2,35,37]$. Model-based methods use mathematical structures-such 
as sets and relations-to model data, and predicate logic to describe the operations on data. A model-based specification typically consists of the description of a state space followed by predicates describing operations which change the state. Although we do not consider state in our type-theoretic approach to specification, the modelbased approach is worth mentioning as it raises general issues about modularity and implementation which are relevant to our work.

$\mathrm{Z}$ is notable for encouraging the incremental construction of structured specifications by using schemas which allow states and predicates to be grouped together and named. $\mathrm{Z}$ allows schemas to import the contents of other schemas, and includes operators to modify and combine schemas. However, there is no mechanism for grouping schemas, and this often leads to problems in structuring large specifications. To solve this problem, [47] proposes an extension to $\mathrm{Z}$ that allows modules containing schemas. The main structuring facility in VDM [21] is procedural and functional abstraction, but [13] and [32] propose extensions to VDM allowing modules containing states and operations, together with import and export facilities to combine modules. [39] describes the incremental specification and development of modules using the refinement calculus, but as yet, there is no formal definition of modules.

Both VDM and the refinement calculus have a refinement relation that allows specifications to be refined to programs. In each case, the programming language is an implementable subset of the specification language, and specifications are refined until they contain only programming language constructs. There are two notions of refinement; procedural refinement, which is concerned with replacing specification statements with programming language statements; and data refinement, which is concerned with replacing fancy data-types, such as sets, with programming language data-types such as arrays. The $\mathrm{Z}$ notation does not define a formal notion of program development, but [22] shows how $\mathrm{Z}$ specifications may be implemented using the refinement calculus. Both [13] and [39] discuss the refinement of modular specifications in VDM and the refinement calculus, respectively.

\subsection{This Thesis}

In this section, we give a brief summary of the contents of this thesis.

Chapter 2 gives the notations used in this thesis for the types and type rules in MartinLöf's type theory. We also define some extensions to the theory, and illustrates the proof style used in this thesis.

Chapter 3 gives some example specifications and modules which illustrate important features of our specification language and its associated implementation language. Most of the features will be justified by the fact that specifications are types; but the 
semantics of specifications are left for later chapters. The features illustrated include the ability to nest specifications, and modules, inside each other; using modules as records; and parameterised specifications and modules.

We give a semantics to specifications and modules by translating them into terms in Martin-Löf's type theory, and Chapter 4 formally defines the terms that may be used as translations for specifications and modules. The definitions are preceded by a detailed review of two other approaches to specifying modules in type theory.

For presentational reasons, Chapter 4 does not give a formal mapping from the syntax of specifications and modules to their translations in Martin-Löf's type theory. That is given in Chapter 5 .

Chapter 6 gives the formal definition of a collection of operators on signatures and computational elements, together with some of their properties; the operators are needed in later chapters to define specification and module operators.

Chapter 7 gives formal definitions for a collection of specification operators, and it gives some of the algebraic properties of the specification operators. Chapter 7 also gives an example that shows how the specification operators can be used to construct large specifications in an incremental style.

Chapter 8 gives a formal definition of refinement for specifications in type theory, and it also gives a collection of refinement laws. Particular attention is paid to laws that allow the piecewise refinement of large specifications. An example of piecewise refinement is also given.

Chapter 9 gives laws for implementing structured specifications. The laws make use of a collection of module operators, also defined in Chapter 9; the module operators are analogous to some of the specification operators. Chapter 9 also gives an example of the piecewise implementation of the example refinement given in Chapter 8 .

In Chapter 10, we discuss some of the outstanding issues raised in previous chapters. Future work is discussed. And we give a summary of the key points of our thesis.

If the reader wishes to skip details of the formal proofs in this thesis-on a first reading, say-then it is also advisable to skip Chapter 5 , as we only use the formal definition of the translation mapping it gives in some of our proofs; Chapter 4 gives an informal description of the translation mapping which should be sufficient for most of our purposes. The purpose of the signature and computational element operators, defined in Chapter 6 , is to define specification and module operators. Therefore, Chapter 6 can be skipped until the reader considers the formal definitions of specification and module operators given in Chapters 7 and 9, respectively; although, we recommend at least reading the informal descriptions of the signature and computational element operators, given in Chapter 6 , before reading Chapters 7 and 9 . 


\section{Chapter 2}

\section{Types and Specifications}

\subsection{The Type Theory}

Although MLT is reasonably well established in the literature, it is constantly being extended, and notational conventions are still fluid. Therefore, in this chapter we give the basic notations and type rules used in this thesis. We assume the reader is reasonably familiar with type theory and this chapter is only intended to give the particular syntactic conventions we use for what we hope are familiar types, terms and rules. We describe some extensions to the theory which may be unfamiliar, but the extensions are relatively straightforward. We also illustrate the proof style used. Most of the notation for types and type rules is borrowed from [44], but proofs are in the natural deduction style exemplified in [3]. It is not our intention to give a complete description of the type theory and its semantics; for this, we refer the interested reader to $[31,44]$.

\subsubsection{A Note About Notation}

We let the capital letters $A, B, C, P, Q$ and $R$ stand for type expressions, and the small letters $a, b, c, e, p, q$ and $r$ (possibly subscripted) stand for type and non-type expressions; both type and non-type expressions may be partial, i.e. they may contain free variables. Variables are $x, y, z$ (possibly subscripted). We let $S P$ (possibly subscripted) stand for types representing specifications, and $m$ (possibly subscripted) stand for modules. The letters $f, g$, and $h$ will normally stand for functions.

The notation $b(x \backslash e)$ stands for an expression $b$ with each free occurrence of variable $x$ replaced by expression $e$. If an expression $b$ contains a free variable- $-x$, say-then we will sometimes rewrite $b$ as $[x] b$ to make this fact evident. The notation $[x] b(e)$ stands for $b$ with each free occurrence of variable $x$ replaced by expression $e:[x] b(e) \equiv b(x \backslash e)$ 
where " $\equiv$ " is definitional equality. When it is clear from the context that $b$ is defined in terms of a free variable $x$, we may abbreviate $[x] b(e)$ to just $b(e)$.

\subsubsection{Judgement Notation}

We make use of the four "judgemental forms" in Martin-löf"s type theory:

$$
\begin{aligned}
& \text { P type } \\
& p \in P \\
& p=q \in P \\
& P=Q
\end{aligned}
$$

The first is read as $P$ is a well-formed type; the second that $p$ has type $P$; the third, that $p$ and $q$ are equal elements of type $P$; and the fourth that $P$ and $Q$ are equal types.

Hypothetical judgements are judgements made under certain assumptions. For example, the judgement $\left[p=_{P} q\right]$ type is true under the assumption that $p \in P$ and $q \in P$, and we write such a judgement as

$$
\left.\llbracket p \in P ; q \in P \triangleright\left[p={ }_{P} q\right] \text { type }\right\rfloor
$$

The square brackets "[" and "]" denote the scope of the context and the symbol " $\triangleright$ " separates the context from the conclusion that can be drawn from it; this notation is due to [3]. In general, a hypothetical judgement has the form:

$$
\llbracket\left[x_{1} \in P_{1} ; \ldots ; x_{n} \in P_{n} \triangleright J\left(x_{1}, \ldots x_{n}\right)\right]
$$

where $J\left(x_{1}, \ldots, x_{n}\right)(n \geq 0)$ stands for a judgement in one of the four judgemental forms given above, and $x_{1} \in P_{1} ; \ldots ; x_{n} \in P_{n}$ represents the context in which the judgement is made.

\subsubsection{The Types}

The types we use are summarised in Table 2.1. Some of the types, such as the propositional types, have been given previously in Table 1.1, but Table 2.1 also contains data-types, such as naturals and lists, which are used specifically for programming. The string, list, set, subset and singleton types were not in the original theory [31]; subset and list types are extensions given in [44, 8], strings are lists of characters, and sets are defined in [3].

The well-formedness of a type expression is given by formation rules. However, we do not make much use of the formation rules of the theory, and the only formation rule of any significance to us is that for the $\sum$-type:

$$
\sum \text {-formation } \frac{P \text { type } \quad[x \in P \triangleright Q(x) \text { type }]}{\sum x \in P . Q(x) \text { type }}
$$




\begin{tabular}{|l|l|}
\hline Type & Type Name \\
\hline $\mathbb{N}$ & Naturals \\
$\mathbb{B}$ & Booleans \\
$\mathbb{S}$ & String type - contains lists of characters \\
$T$ & Unit type - contains the single value $t$ \\
$\phi$ & Empty type - has no elements \\
$P+Q$ & Disjoint sum \\
$\prod x \in P . Q(x)$ & Dependent function space \\
$\sum x \in P . Q(x)$ & Dependent product \\
{$\left[p_{1}=P p_{2}\right]$} & Equality type on $P$ - inhabited iff $p_{1}=p_{2}$ \\
$\operatorname{List}(P)$ & List type - the type of lists of elements of type $P$ \\
$S e t(P)$ & Set type - the type of sets of elements of type $P$ \\
$\{x \in P \mid Q(x)\}$ & Subset type - for elements of type $P$ that satisfy $Q(x)$ \\
$\{p\}_{P}$ & Singleton type - contains the single value $p$ of type $P$ \\
$U_{i}$ & Type Universes $(i>0)$ \\
\hline
\end{tabular}

Table 2.1: Summary of types

\begin{tabular}{|c|c|c|}
\hline Type & Introduction Rule & Elimination Rules \\
\hline Unit & $t t \in T$ & $\frac{x \in T}{x=t t \in T}$ \\
\hline$\phi$ & none & $\frac{r \in \phi}{\phi-\operatorname{elim}(r) \in C(r)}$ \\
\hline$\sum$ & $\frac{p \in P \quad q \in Q(p)}{\langle p, q\rangle \in \sum x \in P . Q(x)}$ & $\begin{array}{l}a \in \sum x \in P . Q(x) \\
\frac{\llbracket x \in P, y \in Q(x) \triangleright b(x, y) \in C(\langle x, y\rangle)]\rfloor}{\operatorname{split}(a, b) \in C(a)}\end{array}$ \\
\hline$\Pi$ & $\frac{\|[x \in P \triangleright q(x) \in Q(x)] \mid}{\lambda x \cdot q(x) \in \prod x \in P \cdot Q(x)}$ & $\frac{f \in \prod(x \in P) \cdot Q(x) \quad a \in P}{f(a) \in Q(x)}$ \\
\hline+ & $\begin{array}{l}\frac{p \in P}{\operatorname{inl}(p) \in P+Q} \\
\frac{q \in Q}{\operatorname{inr}(q) \in P+Q}\end{array}$ & $\begin{array}{l}a \in A+B \\
[x \in A \triangleright c(x) \in C(\operatorname{inl}(x))]\rfloor \\
\frac{[y \in B \triangleright d(y) \in C(\operatorname{inr}(y))]}{\operatorname{when}(a, c, d) \in C(a)}\end{array}$ \\
\hline$=$ & $\frac{p=q \in P}{e q \in\left[p={ }_{P} q\right]}$ & $\frac{c \in\left[p={ }_{P} q\right]}{p=q \in P} \quad \frac{c \in\left[p={ }_{P} q\right]}{c=e q \in\left[p==_{P} q\right]}$ \\
\hline
\end{tabular}

Table 2.2: Introduction and elimination rules for the propositional types 


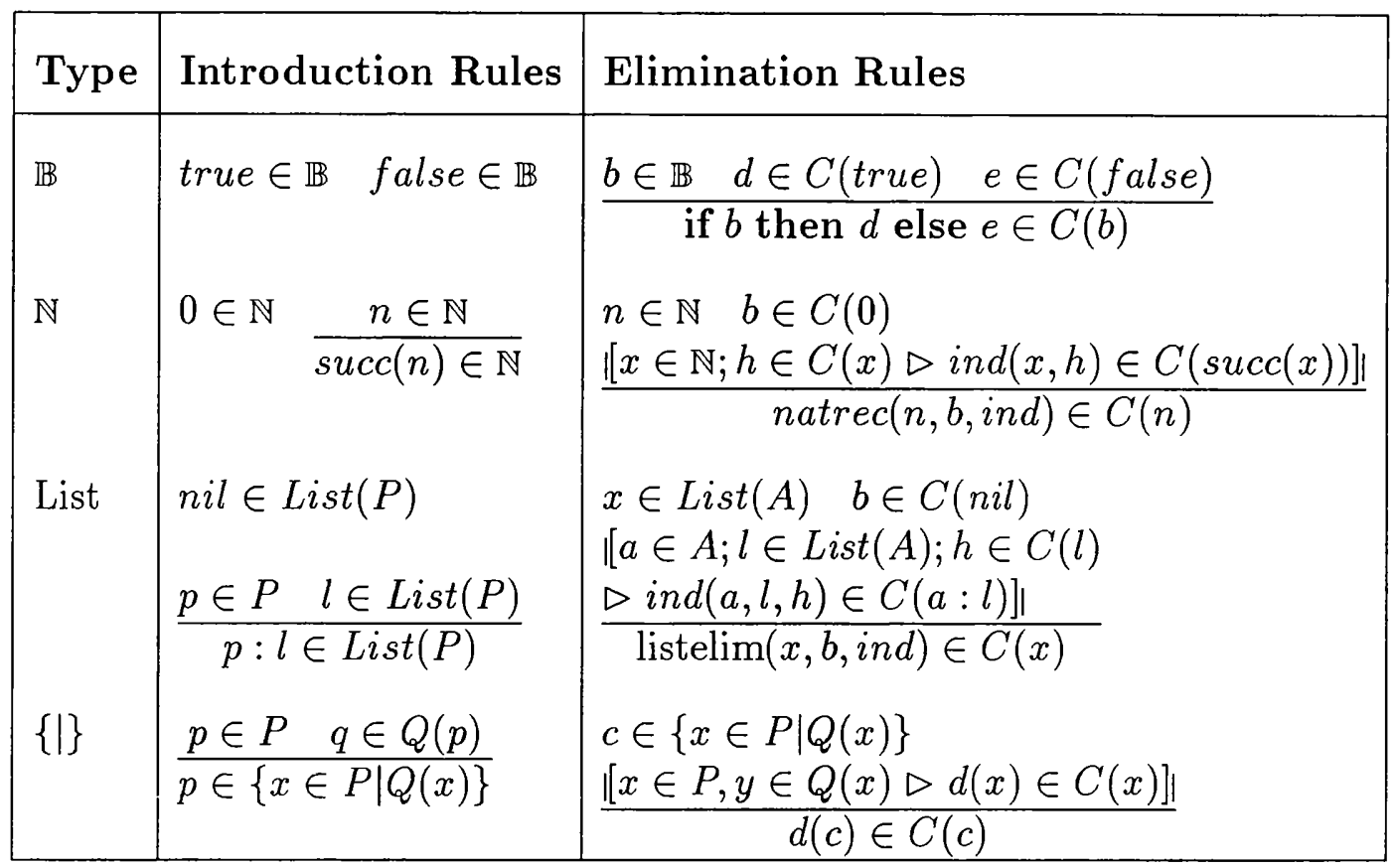

Table 2.3: Introduction and elimination rules for the data types

Tables 2.2 and 2.3 give the introduction and elimination rules for propositional types and data-types, respectively (the separation of the rules for propositional types and data-types is purely to aid presentations and has no other significance). The rules for type universes have been omitted from Tables 2.2 and 2.3, and are described in Section 2.1.4; the rules for the string type, set types and singleton types are also omitted, and described in Section 2.2. The introduction rules show how to form canonical elements of a type. The elimination rules say how to reason about elements of a type (or equally, since meaning is constructive, how to construct functions over elements of a type). The elimination rules associate with a type a so-called noncanonical form. For example, the $\sum$-type has the non-canonical form split; + has the non-canonical form when etc. The non-canonical forms of a type are used to construct functions over elements of the type. Table 2.4 gives the operational semantics of the various non-canonical forms.

The non-canonical form split, associated with the $\sum$-type, is used to define the usual projections on pairs:

\section{Definition 2.1}

$$
\begin{aligned}
& f s t(p) \equiv \operatorname{split}(p,[x, y] x) \\
& \operatorname{snd}(p) \equiv \operatorname{split}(p,[x, y] y)
\end{aligned}
$$

The empty type has no introduction rule as it has no elements. However, in the event that we deduce that $r \in \phi$ for some $r$-an absurdity - then there is an elimination rule which allows us to deduce that the non-canonical expression $\phi$-elim $(r)$ inhabits 


\begin{tabular}{|l|l|}
\hline Type & Computation Rule \\
\hline$\sum$ & $\operatorname{split}(\langle p, q\rangle,[x, y] e)=e(x \backslash p, y \backslash q)$ \\
\hline$\Pi$ & $(\lambda x . e)(p)=e(x \backslash p)$ \\
\hline+ & $\begin{array}{l}\text { when }(\operatorname{inl}(p),[x] c,[y] d)=c(p) \\
\text { when }(\operatorname{inr}(q),[x] c,[y] d)=d(q)\end{array}$ \\
\hline $\mathbb{B}$ & $\begin{array}{l}\text { if } \operatorname{true} \operatorname{then} d \text { else } e=d \\
\text { if } \operatorname{false} \text { then } d \text { else } e=e\end{array}$ \\
\hline $\mathbb{N}$ & $\begin{array}{l}\text { natrec }(0, b,[x, h] \text { ind }) \\
\text { natrec }(\operatorname{succ}(n), b,[x, h] \text { ind })=\text { ind }(n, \text { natrec }(n, b,[x, h] \text { ind }))\end{array}$ \\
\hline List & $\begin{array}{l}\text { listelim }(n i l, b,[x, y, h] \text { ind })=b \\
\text { listelim }(a: l, b,[x, y, h] \text { ind })=\text { ind }(a, l, \operatorname{listelim}(l, b,[x, y, h] \text { ind }))\end{array}$ \\
\hline
\end{tabular}

Table 2.4: Operational semantics for non-canonical forms

all types. The equality type has two elimination rules. The first equality elimination rule states that we are able to construct an object of an equality type whenever we can make the corresponding equality judgement. The second rule rule allows us to deduce that all elements in an equality type are equal to $e q$.

As usual, we shall abbreviate the use of the constructor succ such that $\operatorname{succ}(0) \equiv 1$, $\operatorname{succ}(\operatorname{succ}(0)) \equiv 2$ etc. We use nil for the empty list and $a: l$ denotes the list formed by appending the element $a$ onto list $l$. We often abbreviate lists such as $1: 2: 3:$ nil to $[1,2,3]$. The non-canonical forms natrec and listelim define primitive recursion on naturals and lists, respectively. As the use of natrec and listelim can often be difficult to parse, we usually define recursive functions on naturals and lists in a clausal form. For example, we would define a recursive list function $f \in \operatorname{List}(P) \rightarrow Q$ in a clausal form by:

$$
\begin{aligned}
& f(n i l)=e_{1} \\
& f(a: l)=e_{2}(a, l, h)
\end{aligned}
$$

where

$$
a \in P, l \in \operatorname{List}(P), h=f(l), e_{1} \in Q, e_{2}(a, l, h) \in Q .
$$

Such a definition of $f$ may be written using listelim by:

$$
f(x)=\operatorname{listelim}\left(x, e_{1}, e_{2}(a, l, h)\right)
$$

\subsubsection{Type Universes}

The canonical elements of the type universe $U_{1}$ are all the types given in Table 2.1 save the type universes $U_{i}(i \geq 1)$. We do not give the introduction rules for $U_{1}$ as 
they are too numerous and we shall not need them-the rules are listed in [44]. Each type universe $U_{i}$, for $i>1$, contains all the elements of $U_{i-1}$, and also includes $U_{i-1}$ itself:

$$
\frac{x \in U_{i}}{x \in U_{i+1}} \quad U_{i} \in U_{i+1} \quad(i>0)
$$

There is no widely accepted non-canonical form for type universes, but Nordström et al have introduced urec as the non-canonical form for the type $U_{1}$. urec allows the definition of recursive functions over the structure of types in $U_{1}$-in principle, we could introduce a non-canonical form urec $_{i}$ for each type universe $U_{i}$. However, rather than using urec, we will write functions on types in a clausal form; the reason for this is that the syntax of the urec operator is difficult to use and parse. The clausal forms can be seen as meta-notation which we can always translate into urec expressions. For example, we could define a function $F \in U_{1} \rightarrow U_{1}$ that converts a $\sum$-type to a $\Pi$-type as follows:

$$
\begin{array}{ll}
F\left(\sum x \in P . Q(x)\right) & =\prod x \in P . Q(x) \\
F(P) & =P \quad, \quad P \text { non }-\sum
\end{array}
$$

The clause $F(P)$ is included to make $F$ a total function on types in $U_{1}$; all functions in the type theory must be total, and so we choose to define $F$ as an identity operation when $F$ is applied to a non $\sum$-type. Many of the functions we define on types are intended to be on $\sum$-types only, so that many of our functions on types will take a similar form to $F$ above.

\subsection{Extensions to Type Theory}

A feature of Martin-Löf's type theory is that it is amenable to extension by the addition of new types. Backhouse et al [3] describe a systematic approach to adding new types. In this section, we use the approach described by Backhouse et al to define some new types that we will require.

\subsubsection{Characters and Strings}

The Character type is denoted Char. The intended elements are alphabetical characters and mathematical symbols; Char is analogous to the char type in Pascal. We write characters inside single quotes, and members of Char include ' $a$ ', ., , $z$ '; 'A', . . 'Z'; '0', .., '9'; '十', ' $\oplus$ '. We could add more characters to Char, but those we have listed are sufficient for our purposes. We assume the existence of an equality function, _ $=_{-} \in$ Char $\rightarrow$ Char $\rightarrow \mathbb{B}$, on characters. The type rules for Char are omitted, as we do not make use of them. For the interested reader, Char is defined 
as an enumeration, or finite, type as described in [44] and [31], and its non-canonical form is a Pascal style Case statement over its elements; we use the Case statement to define the equality function on Char, mentioned above.

The character type is used to define the string type, denoted $\mathbb{S}$, whose elements are lists of characters:

$$
\mathbb{S} \equiv \operatorname{List}(\text { Char })
$$

Instead of writing strings as lists, we use the convention of writing strings within single quotes; for example, 'hello' $\equiv$ ['h', 'e', 'l', 'l', 'o']. The empty string is written ', and " $\equiv$ nil.

\subsubsection{Set Types}

The set type is an extension to the theory introduced by Backhouse et al [3]. We do not give a complete explanation of set types as they are only used in examples where the usual intuitive understanding of sets is sufficient. The type of a set containing elements of type $P$ is written as $\operatorname{Set}(P)$. The introduction rules for sets introduce an empty set \{\} , and $p:: s$ denotes the set $s \in S e t(P)$ with $p \in P$ added to $s$ :

$$
\{\} \in \operatorname{Set}(P) \quad \frac{p \in P \quad s \in \operatorname{Set}(P)}{p:: l \in \operatorname{Set}(P)}
$$

The non-canonical from for sets is called setelim, and its operational semantics are similar to listelim.

$$
\begin{array}{ll}
\operatorname{setelim}(\{\}, e,[p, s, h] \text { ind }) & =e \in C(\{\}) \\
\operatorname{setelim}(p:: s, e,[p, s, h] \text { ind }) & =\operatorname{ind}(p, s, \operatorname{setelim}(s, e,[p, s, h] \text { ind })) \in C(p:: s)
\end{array}
$$

where

$$
p \in P, s \in \operatorname{Set}(P), \| x \in \operatorname{Set}(P) \triangleright C(x) \text { type }], h \in C(s)
$$

In fact, sets are similar to lists, except that setelim has restrictions to ensure that sets are independent of the number of occurrences of any element appearing in their construction; and sets are independent of the order in which elements appear in their construction. The restrictions on setelim are given by the following rules which are additional computational rules for sets:

$$
\begin{aligned}
& \operatorname{setelim}(p:: p::\{\}, e,[p, s, h] \text { ind })=\operatorname{setelim}(p::\{\}, e,[p, s, h] \text { ind }) \\
& \operatorname{setelim}(p:: q:: s, e,[p, s, h] \text { ind })=\operatorname{setelim}(q:: p:: s, e,[p, s, h] \text { ind })
\end{aligned}
$$




\subsubsection{Singleton Types}

We extend the type theory with a singleton type constructor $(\{\})$ that enables the definition of types containing single elements. For example, $\{\text { true }\}_{\mathbb{B}}$ is the type containing the single element true from the type $\mathbb{B}$; and $\{[1,2,3]\}_{\text {List }(\mathrm{N})}$ is the type containing the single list $[1,2,3]$. The element of a singleton type must be a member of an existing type; the tag on a singleton type allows us to deduce the base type of an element of a singleton type. The formation, introduction and elimination rules for the singleton type are given below.

$$
\frac{P \text { type } p \in P}{\{p\}_{P} \text { type }} \quad \frac{P \text { type } p \in P}{p \in\{p\}_{P}} \quad \frac{x \in\{p\}_{P}}{x=p \in P}
$$

Remark The type $\{p\}_{P}$ is not equal to the subset type $\left\{x \in P \mid\left[x=_{P} p\right]\right\}$. The elimination rule for the subset type does not allow us to deduce that $e=p \in P$ from $e \in\left\{x \in P \mid\left[x=_{P} p\right]\right\}$. We can approximate a singleton type $\{p\}_{P}$, using the type $\sum x \in P .\left[x=_{P} p\right]$. The sole element of $\sum x \in P .\left[x={ }_{P} p\right]$ is the pair $\langle p, e q\rangle$, so it is not equivalent to $\{p\}_{P}$, but is a reasonable approximation if we ignore the extra component $e q$. The addition of the singleton type can therefore be regarded as just a convenience that avoids the need to deal with pairs; from our approximation of singleton types, we can deduce inference rules similar to the three given above.

\subsection{Proof Style}

The inference rules of the type theory admit of a natural deduction style for making proofs of judgements. Natural deduction style proofs can be written in a very systematic manner, and there are several recognised styles for writing them. In this thesis we adopt the proof style given by Backhouse et al in [3], which we illustrate by giving the proof of the proposition:

$$
(P \rightarrow Q) \wedge(Q \rightarrow R) \rightarrow(P \rightarrow R)
$$

This proposition corresponds to showing that $\rightarrow$ is transitive. We intend to prove the proposition true constructively by showing the following:

$$
\lambda p . \lambda x .(\operatorname{snd}(p))((f s t(p))(x)) \in(P \rightarrow Q) \wedge(Q \rightarrow R) \rightarrow(P \rightarrow R)
$$

The derivation is given in Figure 2.1. The line numbers, and comments in quotes are not part of the derivation but are intended to aid the reading of the proof. Each comment gives the assumptions and inference rules used to make the judgement that follows the comment. Proofs make extensive use of hypothetical judgements which are delimited by square brackets: assumptions appear before the " $\triangleright$ " symbols, and consequences drawn from the assumptions appear to the right of the " $\triangleright$ " symbol. 


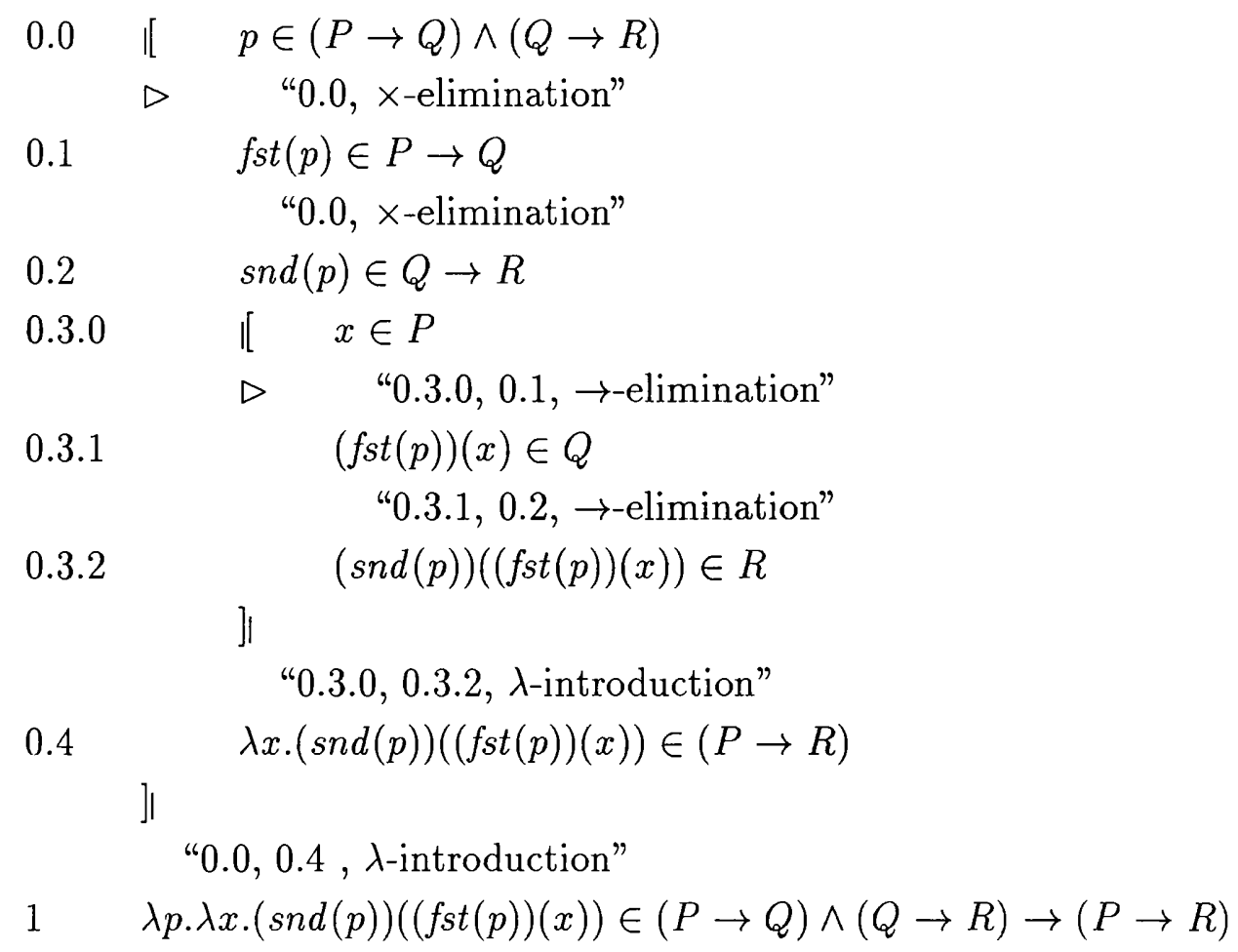

Figure 2.1: Derivation of $\rightarrow$ transitivity

We now give a brief explanation of the derivation given by Figure 2.1. The proposition we are trying to prove is an implication where the antecedent is $(P \rightarrow Q) \wedge(Q \rightarrow R)$ and the consequent is $P \rightarrow R$. Step 0.0 begins by assuming that $p$ is a proof (member) of the antecedent; using this assumption it is our aim to prove the consequent. Looking ahead to steps 0.4 and 1 , having constructed an element of $P \rightarrow R, \lambda$-introduction can be used to complete the derivation. The assumption $p$ in step 0.0 is a pair of type $(P \rightarrow Q) \wedge(Q \rightarrow R)$. From that assumption we may conclude (steps 0.1 and 0.2 ) that $f s t(p)$ and $s n d(p)$ are elements of type $P \rightarrow Q$ and $Q \rightarrow R$, respectively-we use $\times$ elimination to mean the application of either projection function. Now, in steps 0.3.0, 0.3.1 and 0.3.2 we construct an element of type $P \rightarrow R$. First (step 0.3.0) we assume that $x$ is an element of $P$. Using that assumption we may conclude (step 0.3.1) that applying $f s t(p)$ to $x$ ( $\rightarrow$-elimination) yields a value of type $Q$, and hence, in step 0.3.2, applying $s n d(p)$ to $(f s t(p))(x)$ gives a value of type $R$. Step 0.4 now follows by $\lambda$ elimination-note the discharge of assumption 0.3.0. Finally, we obtain the desired conclusion (step 1) by discharging the initial assumption 0.0 . 


\section{Chapter 3}

\section{Typeful Specifications}

\subsection{Introduction}

In this chapter, we outline some features of our specifications and modules. Adopting a type-theoretic view of modules and their specifications allows us to use many of the features of type theory when making specifications, and we illustrate the use of some of those features. We proceed mainly by example, without trying to describe the mathematical underpinnings in any detail. Of course, a formal semantics is important for the purposes of formal reasoning about specifications and modules, but the issues raised here can be understood without a detailed knowledge of the semantics of specifications; we leave the semantics until the next chapter.

We give an example of the use of local components to make specifications and modules. The other features illustrated in this chapter arise as a direct result of interpreting specifications as types. We show that we can use the built-in types and operations of Martin-Löf's type theory to help make specifications and their implementations; we claim such specifications are model-based since they use the built-in types and operations. We also give some examples of hierarchical specifications; these are specifications that have other specifications nested within them. We also show how specifications can be used as data-types, and how modules can be used as first class values within specifications and modules.

In the following, when we give examples of a module satisfying a specification we will omit to prove that the module satisfies the specification; proofs are omitted until we discuss the formal semantics of specifications, in the next chapter. Furthermore, we will omit witnesses from modules, replacing them by "...". 


\subsection{Local components}

When we make a specification, we often specify auxiliary components whose sole purpose is to help specify other components. These auxiliary components are not intended to be available for use by other "client" specifications; they are local components intended for use within a specification. Local components are useful. Firstly, we can use them to break up the specifications of complex operations into more manageable pieces. Secondly, we can "hide" components by making them local, and this allows us to modify and reuse old specifications to make new ones; we can hide components not needed in the new specifications. Thirdly, by specifying a component as local, we are saying that it is not intended to be part of the "interface" to the specification. This last point is important, as it tells an implementor which components must appear in an implementation, and which components they are free to change or remove. Our specification language, and its associated implementation language, contain features that allow a specifier to indicate which components are local. Components intended to be part of the interface of a specification are called visible components.

\subsubsection{An example of local components-The Mean Module}

We distinguish local components in a specification by putting a fat dot $(\bullet)$ in front of their declaration in the signature; components without a fat dot are visible. Consider the specification Mean, given in Figure 3.1. Mean specifies operations for calculating the mean of a collection of naturals. Data is intended to be a type for samples of data where the samples consist of a collection of naturals; so Data might be implemented by $\operatorname{Bag}(\mathbb{N})$, List $(\mathbb{N})$ etc. clear is an empty sample. The function sum returns the sum of a sample of data; sum is a local component whose sole purpose is to help define the component mean. The function size gives the number of values in a sample; enter is used to add a new value to a sample; and mean takes the mean of a sample. mean is a dependent function: for a non-empty sample the result of mean is a natural, but if we try and take the mean of an empty sample then mean returns the string "error". We note in passing that mean is an example of an operation whose type is dependent on a non-type component, namely, the function size. It is unfortunate 


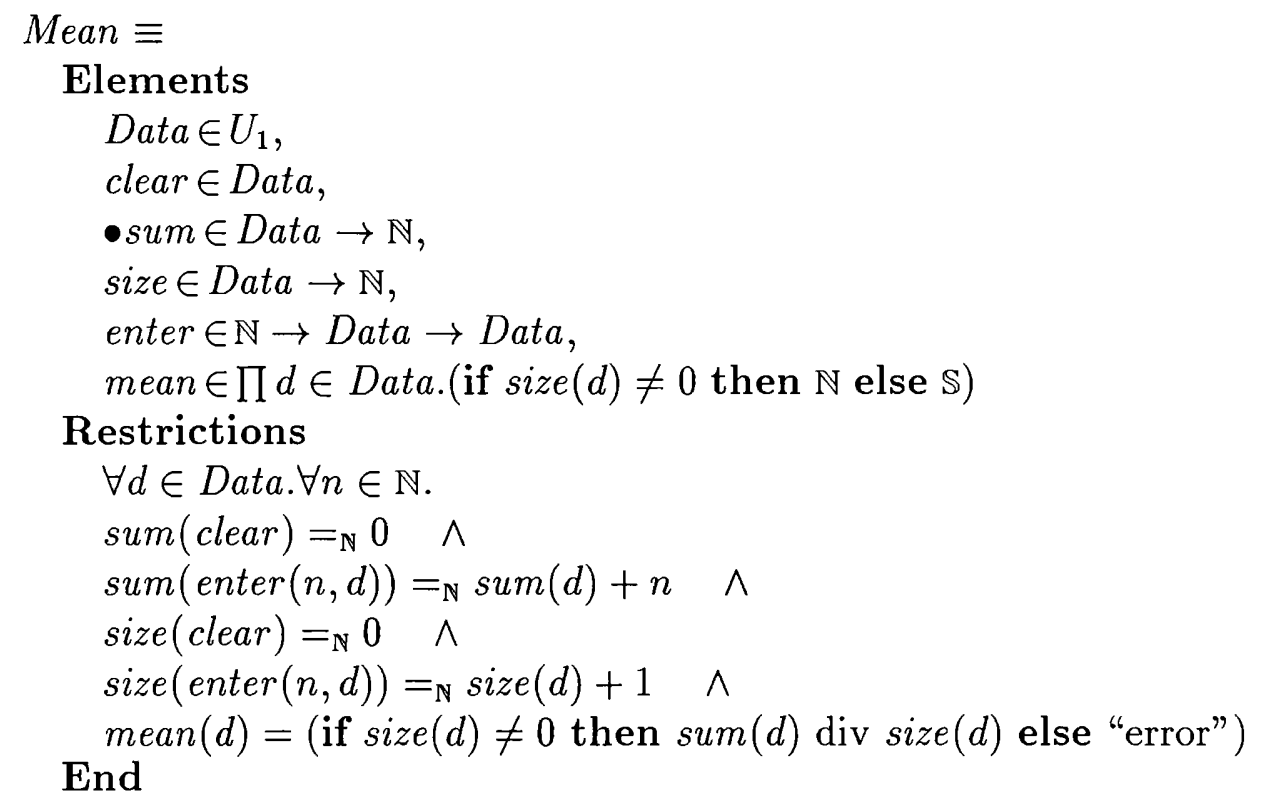

Figure 3.1: The Mean specification

that the expression size $(d) \neq 0$ is repeated in both the type of Mean and in the axiom that specifies mean. We can avoid such repetition by redefining the type of mean as follows:

$$
\begin{gathered}
\text { mean } \in \prod d \in \text { Data.if } \operatorname{size}(d) \neq 0 \text { then } \\
\{\operatorname{sum}(d) \operatorname{div} \text { size }(d)\}_{\mathrm{N}} \\
\text { else } \\
\{\text { "error" }\}_{\mathbb{S}}
\end{gathered}
$$

The new type for mean completely specifies the behaviour of mean since the only value in the singleton type $\{\operatorname{sum}(d) \text { div size }(d)\}_{\mathrm{N}}$ is $\operatorname{sum}(d)$ div size $(d)$; and the only value in type $\{\text { "error" }\}_{\mathbb{S}}$ is "error". Consequently, there is no need for the final axiom in the restrictions of Mean.

When we implement a specification we are obliged to implement both its visible and local components; we will justify this obligation in Section 4.11. The implementations of local components are distinguished by a fat dot before their name. Figure 3.2 gives an implementation of Mean, called MeanModule, containing implementations for all the components specified by Mean, including the local component sum. MeanModule represents data samples as pairs of naturals: the first component of such a pair is the sum of all the elements in the sample, the second component is the size of the sample.

The main difference between the visible and local components in a module is that clients cannot use dot notation to refer to local components; this shields clients from future changes to local components. An attempt to refer to a local component by dot notation just returns the unit value $t t \in T$; for example, MeanModule.sum $=t t$. Visible components may be referred to by dot notation in the usual way. 


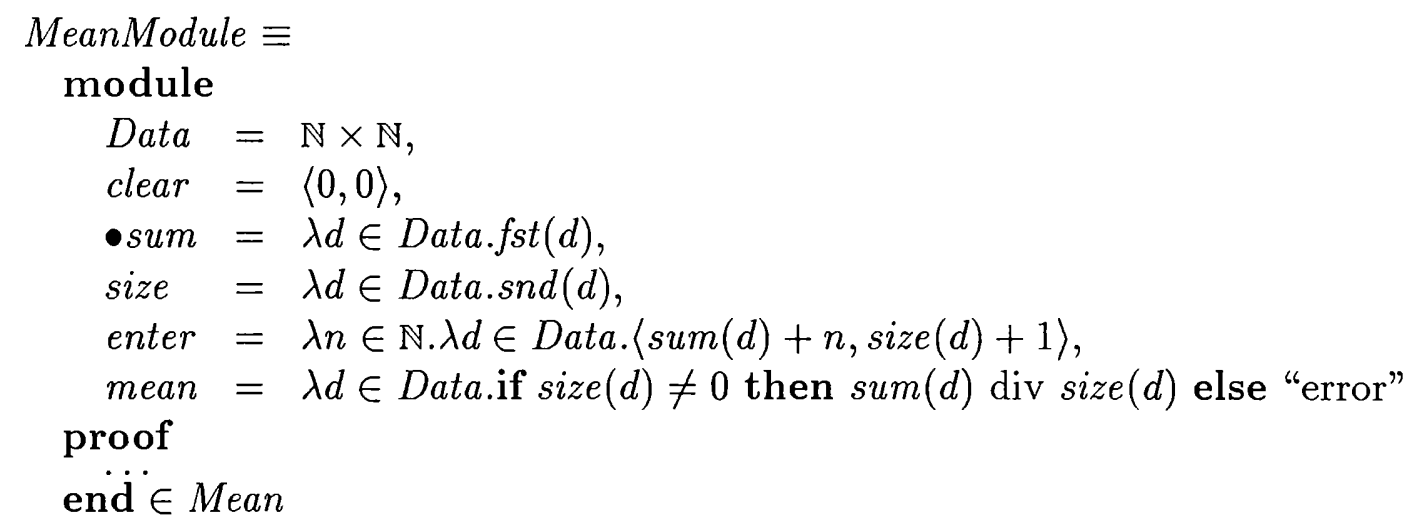

Figure 3.2: An implementation of Mean

\subsection{Model-Oriented Specifications}

The specifications given in the previous sections specify abstract data types (ADT's); that is, they specify modules containing type components - that are equivalent to sorts in algebraic languages-together with operations on the types. Such specifications are similar to algebraic specifications. However, our specification language is not an algebraic specification language - as we shall see later, its semantics is given in terms of type theory-so that we are not restricted to purely algebraic style specifications.

One difference between our language and algebraic specification languages is that we provide many built-in types such as $\mathbb{N}, \mathbb{B}$, List, Set etc; algebraic specification languages do not have built-in types. The built-in types can be used as "models" to help specify modules. In particular, we can make specifications by using the predefined operations that built-in types come equipped with. In this section, we give two examples of a model-oriented approach to specifying modules.

\subsubsection{A model-oriented axiomatic specification}

The specifications we have seen so far have been given in a purely property-based style similar to algebraic specifications. However, it is often unclear in a propertybased specification whether its restrictions actually specify the properties we desire. The lack of clarity stems from the fact that property-based specifications are overly abstract; they avoid being biased towards any one choice of implementation for datatypes. In our opinion, specifying an actual value, or model, for data-types often leads to specifications that are easier to make and understand. Our specification language allows us put constraints on type components declared in the signature of specifications, and so specify a model for type components.

Consider the specification Catalogue 2 given in Figure 3.3. Catalogue2 is intended to be a model-oriented version of the book catalogue specification, Catalogue, which 


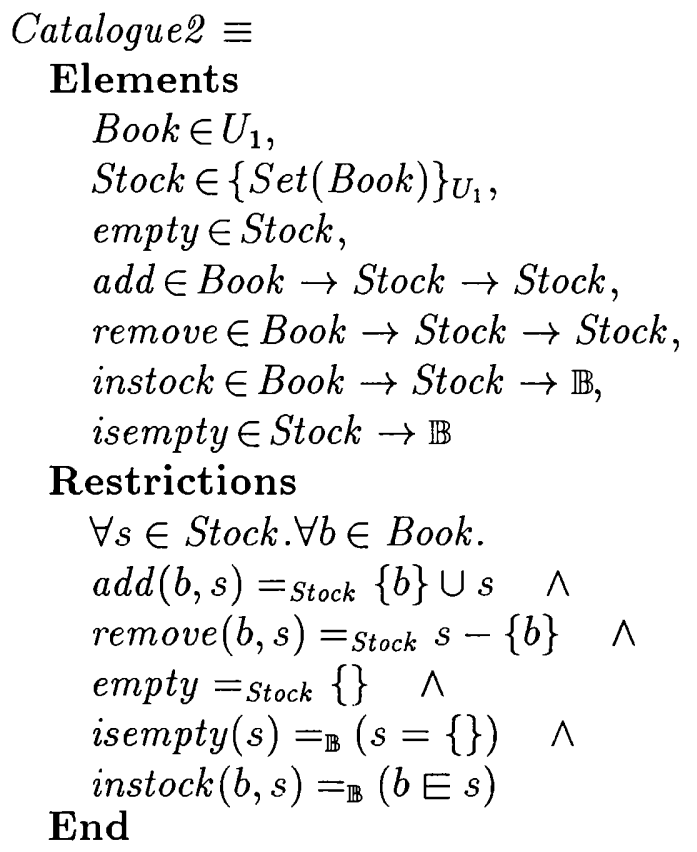

Figure 3.3: A model-oriented catalogue specification

$$
\begin{aligned}
& \{b\} \equiv b::\{\} \\
& s \cup t \equiv \operatorname{setelim}(s, t,[x, y, h] x:: h) \\
& b \in s \equiv \operatorname{setelim}(s, \text { false, }[x, y, h] \text { if }(x=b) \text { then true else } h) \\
& s-t \equiv \operatorname{setelim}(s,\{\},[x, y, h] \text { if }(x \in t) \text { then } h \text { else } x:: h)
\end{aligned}
$$

Figure 3.4: Some standard set operations

was defined in a property-based style in Figure 1.2. Catalogue2 constrains the type component Stock to be the type Set(Book). This is done by specifying Stock to be of type $\{\operatorname{Set}(B o o k)\}_{U_{1}}$ which is a kind containing the single type $\operatorname{Set}(B o o k)$. Hence, we may deduce that $S t o c k=S e t(B o o k)$, and we exploit this to make the restrictions. In contrast, Catalogue declares Stock to have the type $U_{1}$, which tells us that Stock is a type, but tells us nothing more about Stock values. Restricting Stock to the type $\operatorname{Set}(B o o k)$ allows us to use set operations on Stock values. Figure 3.4 gives the definitions of some set operations such as set union (U), set membership (E) and set extraction (-). The set operations behave as we would expect, and they are used to specify the restrictions on Catalogue2.

In our view, the restrictions on Catalogue 2 are easier to understand than those on Catalogue. Of course, such a comparison is subjective-in part, our view is due to a familiarity with sets and their properties.

Remark We have observed that when we make a model-oriented specification it often has fewer axioms than a property-based specification of the same module; for 


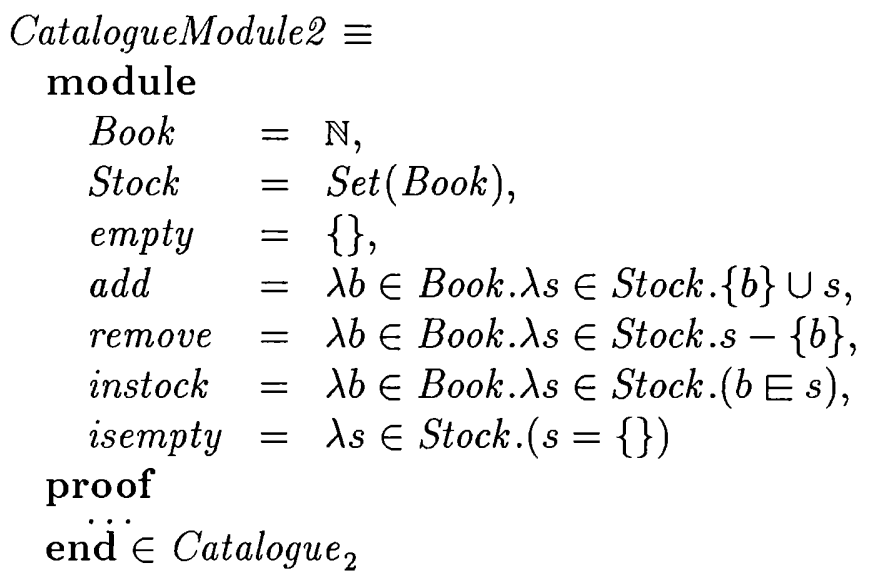

Figure 3.5: An implementation of Catalogue2

example, Catalogue ${ }_{2}$ has 6 axioms, in comparison to 9 in Catalogue. We have also observed that as we add new components to a specification, the number of axioms appears to grows quadratically if it is property-based and linearly if it is modeloriented. We stress that these observations are based on our own experience of making specifications and are not justified by any theoretical, or experimental evidence.

The usual argument against choosing a model for data-types is that the specification is then biased in favour of implementations that use this model, resulting in fewer choices of implementations for the specification. For example, Figure 3.5 gives the obvious implementation that Catalogue2 is biased towards. However, although Catalogued specifies Stock to be $\operatorname{Set}($ Book) it is possible to develop implementations of Catalogue2 which do not implement Stock as $\operatorname{Set}(B o o k)$. Using the technique of data refinement we can change the data-types used by a specification; this allows us to develop modules that use models other than the one given in their specification. For example, we could change the representation of Stock from a set to a list, so that in fact CatalogueModule, defined earlier in Figure 1.3, is also an implementation of Catalogue2. We discuss data refinement in Chapter 8. So apparent bias should not be seen as a problem.

\subsubsection{A Non-ADT}

Not all specifications we define are ADT's. We often make specifications that specify no type components at all. Such specification usually consist of a collection of operations that are defined in terms of the built-in types of Martin-Löf's type theory. If a specification only specifies a small number of components then it is often easier to make the specification in terms of the built-in types. Some modules naturally lend themselves to specification in terms of the built-in data types; for example, modules containing mathematical operations; in such cases we should feel free to use the built-in types. For example, the specification Maths, given in Figure 3.6, specifies 


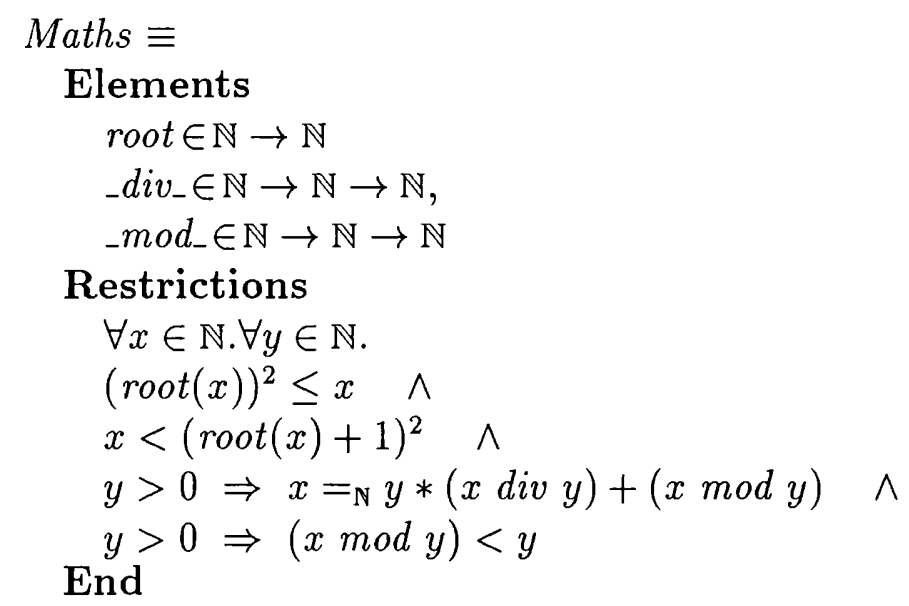

Figure 3.6: The specification of a maths module

an integer square root function, as well as the functions div and mod which give the quotient and remainder of integer division, respectively. Both div and mod are infix operators and we denote this by putting underscores around them (e.g. _div_). Maths contains no type components, only operations defined in terms of the type $\mathbb{N}$.

We do not wish to give the impression that a model-oriented style is always to be preferred over a property-based style. Some problems are more easily expressed in a property-based style, whilst others lend themselves to a model-oriented style; the specifier should be free to choose which style is best for a particular problem. It is interesting to note that although we have called Maths a model-oriented specification, its restriction is in a property-based style-the restrictions are not in the style "root = ..." as used in Catalogue2. In fact, in the case of Maths, defining an axiom of the form "root $=\ldots$. would require introducing a level of algorithmic detail not appropriate to a specification. Clearly, there are sometimes advantages in combining the model and property-based approaches, using a type-theoretic approach to specification allows us to do this.

\subsection{Nested Specifications and Modules}

One of the features of our specification language is that we can nest specifications inside other specifications, and modules inside other modules. We nest specifications by using specifications as the types of components within other specifications. We can do this because specifications are types. The implementations of nested specifications are modules which have other modules as some of their components. Nesting plays a useful role in structuring specifications and modules. In particular, nesting allows us to make new specifications and modules by using components specified in other specifications and modules. 


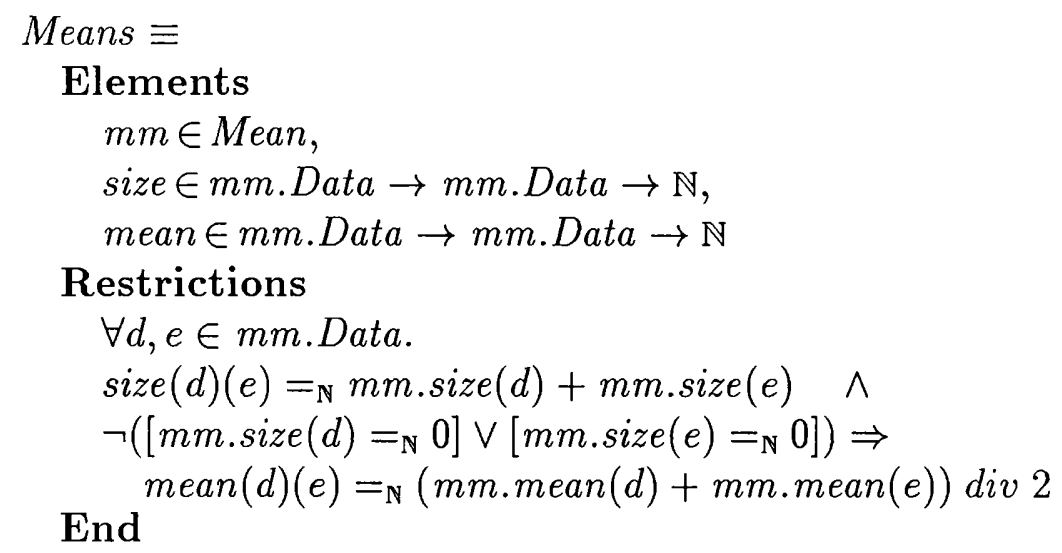

Figure 3.7: The Means specification

\subsubsection{A Nested Specification}

The specification Mean, which we defined earlier in Figure 3.1, specified operations to find the size and mean of a single sample of natural numbers. Suppose we wish to collect two samples-which might be generated by running an experiment twice, say - and calculate the combined mean and size of the two samples. We can use Mean to make a new specification that specifies operations to calculate the size and mean of two data samples. Such a specification, called Means, is given in Figure 3.7. Means contains an example of specification nesting: we use Mean as a type inside Means to specify component $m m$ to be a module that satisfies Mean. The operation mean takes two data samples and returns the combined mean of both samples. We deliberately say nothing about the result of mean if either of the data samples is empty; we leave such a decision for an implementor. We use the components in $m m$ to specify both the type and behaviour of mean; for example, the domain type of mean is given using the type mm.Data. The function size returns the combined size of two data samples.

\section{Scope and Visibility of Names}

Components specified at different levels of nesting in specifications may have the same names. For example, the name size occurs at two different levels in Means: it occurs in the domain of Means, and within the constituent module $\mathrm{mm}$. Similarly, mean occurs in the domains of Means and $m m$. To avoid confusion, we need scope rules that define which declaration of a name is in scope at any particular point. The scope rules for nested specifications will be defined to be similar to the scope rules of a block-structured programming language, like Pascal. Uses of component names are bound to their inner-most (most recent) definition. For example, uses of the name size in Mean, the type of $m m$, are bound to the declaration of size in the signature of Mean, rather than the outer declaration of size in the signature of Means. 
Although many specification languages, such as Z and ASL, allow the nesting of specifications, most simply import all the components of one specification into another without introducing any form of hierarchy. In other words, all components within a specification, regardless of nesting, are always in scope. This means that all components in a $\mathrm{Z}$, and ASL, specification occupy the same name-space, so that name-clashes become a problem as specifications increases in size. However, the style of nesting we have described is hierarchical as it introduces different scopes, and so, components with the same name can co-exist.

\subsubsection{A Nested Module}

Modules may also contain nested modules; by nested module we mean modules which are components within other modules. As an example, consider the module MeansModule defined in Figure 3.8. MeansModule is an implementation for the specification Means with explanation following. The component $m m$ is a module that satisfies Mean; recall that the type of $m m$ is specified to be Mean. We can implement $\mathrm{mm}$ directly using MeanModule which we gave earlier-in Figure 3.2-as an example of an implementation for Mean. We use the components Data, size and mean from $\mathrm{mm}$ to implement the functions size and mean.



Figure 3.8: A module satisfying Means

The scope rules for nested modules are similar to those for nested specifications. For example, the function size used in the definition of function mean in $m m$, refers to size defined in $m m$, rather than the size defined in MeansModule. To refer to the components in a nested module we must use dot notation twice. For example, MeansModule.mm.enter is the function enter from module $\mathrm{mm}$ in MeansModule.

As an interesting aside, notice that by reusing Mean to make Means, we are also able to reuse the module MeanModule to make MeansModule. It is often the case 
that if we reuse a specification to make a new specification, then we can also reuse its implementations to implement the new specification.

\subsection{Specifications as Record Types}

Since specifications are types, we can use them just as we would use other types. In this section, we give an example of using specifications as record types. We consider taking our library catalogue example a little further and specify a module of operations on books; for example, the module may contain operations to view the author or title of a book. We model books as records containing a book number, together with author and title fields. This is done by using the specification BookRec, given in Figure 3.9, as a type for books. BookRec represents the isbn number of a book as a natural number, and the author and title by strings. The intended use of BookRec does not require putting any restrictions on its components, so that its restriction is specified to be the unit type $T$. Of course, we could specify that the author and title should be limited to a fixed number of characters; for example, we could limit them to 20 characters using the following restriction: $\sharp$ author $=_{\mathrm{N}} 20 \wedge \sharp t i t l e={ }_{\mathrm{N}} 20$.

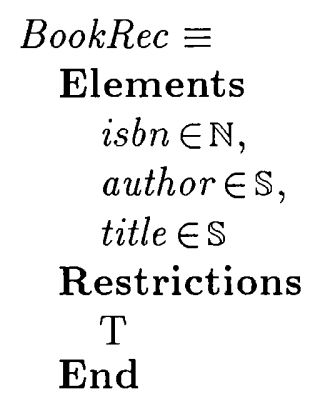

Figure 3.9: A type for book values

We use BookRec to make the specification Books which specifies a book module; Books is given in Figure 3.10, with explanation following. As stated earlier, we specify the type Book to be BookRec; note the use of the singleton type constructor to specify that $B o o k=B o o k R e c$. The function mkBook takes a book number, author and title, and returns a Book value. $m k B o o k$ is an example of a function that returns a module as its result; the module is used like a record. The function view takes a module denoting a book value and extracts its title; view is an example of a function whose domain is a specification.

The operations specified in Books could have been added as extra operations to Catalogue or Catalogue2 in order to make a library specification. However, a more modular solution is to develop a separate specification for books-as we have done above-and then compose this specification with Catalogue or Catalogue2 in some way. To allow 


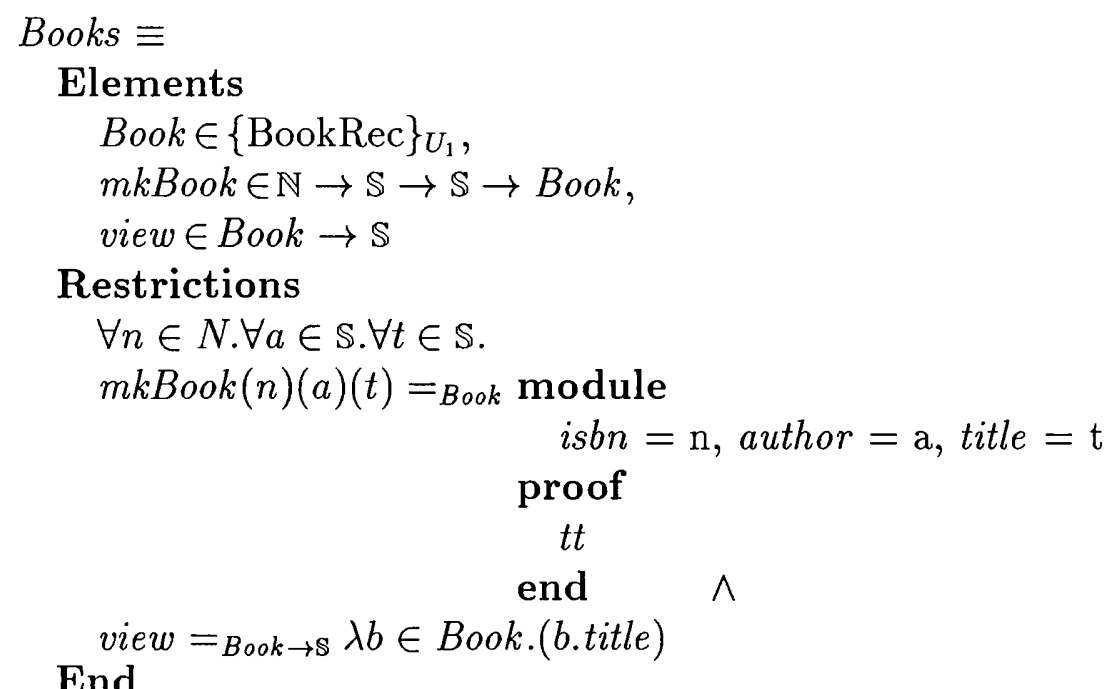

Figure 3.10: The specification of a book module

such modular development of specifications requires specification building operations. We discuss such operations later, in Chapter 7.

However, even with our, at present, limited repertoire of structuring features, we can still package Catalogue and Books into a single specification by using nesting. Figure 3.11 gives the specification of a module that contains a book module and a catalogue module. Recall that both Books and Catalogue contain a type named Book. The restriction on Catalogue3 ensures that the Book components in bookmodule and catalogue are both implemented using the same type; this ensures that Book values used in bookmodule are compatible with those used in catalogue. Without the restriction on Catalogue3 we would be free to implement the Book component in bookmodule independently from the Book component in catalogue. This would be undesirable, since Book values generated by operations in bookmodule might no longer be compatible with Book values used in catalogue. The restriction on Catalogue3 can be seen as a 'gluing' invariant between Catalogue and Books.

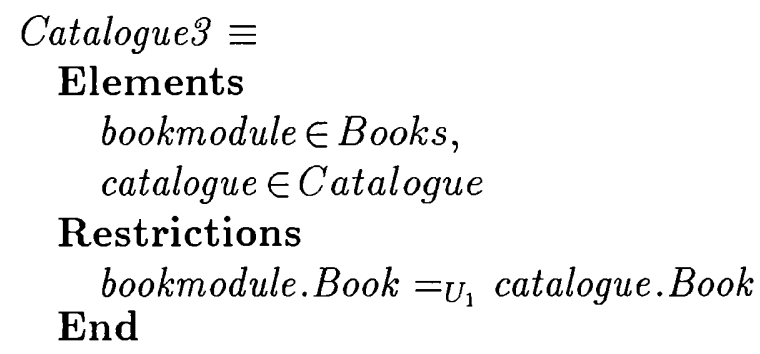

Figure 3.11: The specification of a book catalogue 


\subsection{Parameterisation}

One of the advantages of using type theory as a framework for specification is that types, and specifications, can be treated as first class values. This allows us to define functions returning specifications; such functions are parameterised specifications. Since types are first class values, we can parameterise specifications by types to produce generic specifications that may be instantiated in different ways. By using specifications as types, we may also parameterise specifications with respect to modules, and this provides another useful means of structuring specifications.

The formal parameters of a parameterised specification may be any typed value allowed in the type theory; this includes values such as integers and boolean, as well as data types, modules and specifications themselves. The body of a parameterised specification is simply a specification defined in terms of some formal parameter names. In this section we only consider parameterised specifications whose formal parameters are data types and values; a discussion of specifications as formal parameters is left until Chapter 7 since the meaningful use of specifications as parameters requires the use of specification operators.

\subsubsection{Generic Specifications}

The specification Sequence, given in Figure 3.12, is a parameterised specification, and is a generic specification for sequences. The formal parameter elem $\in U_{1}$ indicates that Sequence takes a type as argument: elem is the type of the elements in the sequences that Sequence specifies. Sequence defines some standard sequence operations: empty is the empty sequence; the infix operator $\left\langle_{-}\right\rangle$makes a unit sequence; the binary infix operator _ $\bullet$ _ concatenates two sequences; head returns the first elements in a sequence; and tail yields all but the first element in a sequence. Instantiated instances of Sequence are specification obtained by substituting the formal parameter elem by an actual parameter, in the body of Sequence. For example, Sequence $(\mathbb{N})$ specifies sequences of naturals; Sequence $(\mathbb{B})$ specifies sequences of boolean etc.

Once instantiated, Sequence can be implemented like any other specification. As an example, we consider an implementation of Sequence $(\mathbb{N})$. List types are available as a standard type in Martin-Löf's type theory. Consequently, we can define a module satisfying Sequence $(\mathbb{N})$ using the type $\operatorname{List}(\mathbb{N})$ to implement $S e q$. We can use the standard list operators (H), nil, $t l$ and $h d$ to implement the operations defined by Sequence. Such an implementation-named SeqImp - is given in Figure 3.13. Since Sequence does not specify a value for head(empty) we have the freedom to choose, and in SeqImp we define it to be 0. We could have implemented Sequence as a 




Figure 3.12: A Parameterised Specification of Sequences

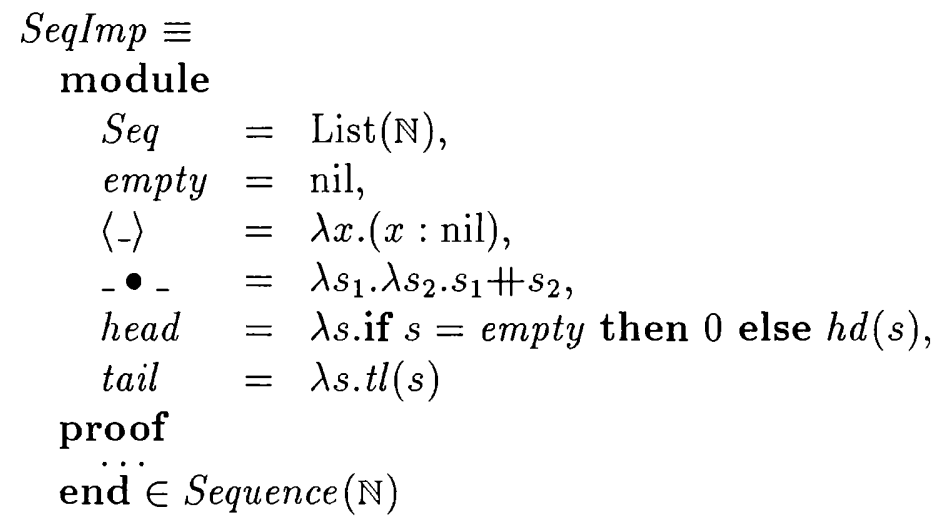

Figure 3.13: An Implementation of Sequence $(\mathbb{N})$

parameterised module, but we leave the discussion of parameterised modules for the next section.

\subsubsection{Parameterisation for Structuring}

The specification ParMeans, given in Figure 3.14, is another example of a parameterised specification. ParMeans is similar to the specification Means, given previously in Figure 3.7; both specifications contain operations allowing the calculations of the means of collections of data. The formal parameter $m m$ records the fact that ParMeans is dependent on a module satisfying the specification Mean where Mean was given previously in Figure 3.1. 


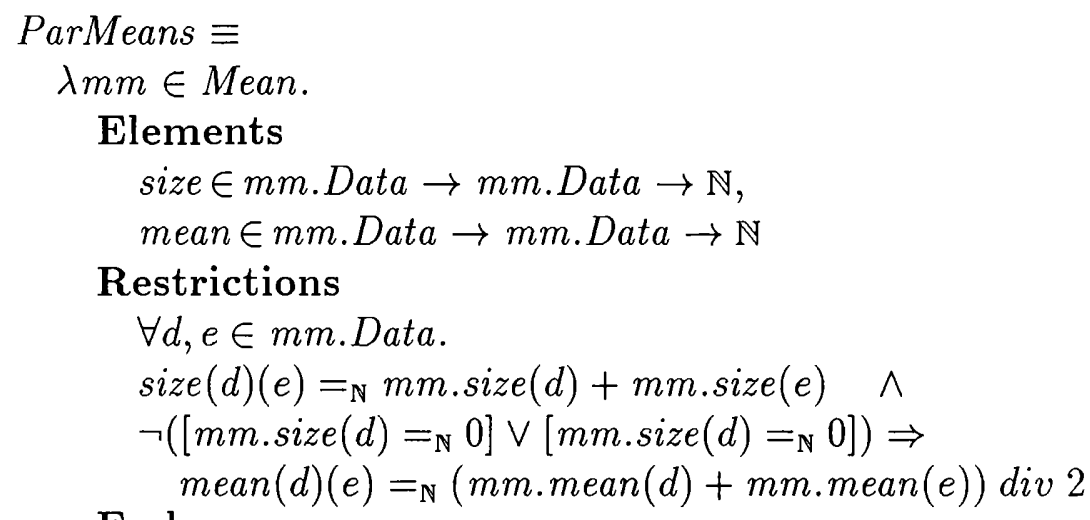

Figure 3.14: A parameterised version of Means

ParMeans takes a module $m m \in$ Mean as its argument and uses it to make the body of ParMeans. In contrast to Means, we do not introduce $m m$ into the signature of ParMeans; this leads to ParMeans having a cleaner signature than Means. ParMeans is an example of where parameterisation is used to structure a specification. ParMeans is not intended to be instantiated with many different parameters, but will instead be instantiated with whatever module we finally choose as an implementation for Mean, the type of the formal parameter of ParMeans.

We choose to implement ParMeans as a parameterised module; a parameterised module is a function that returns a module as its result. Figure 3.15 gives an example of one possible implementation of ParMeans. ParMeansModule is a function that takes any module $m m \in$ Mean and returns a module satisfying ParMeans $(\mathrm{mm})$ (i.e. $\|[m m \in$ Mean $\triangleright$ ParMeansModule $(m m) \in \operatorname{ParMeans}(m m)])$. So, whatever module we finally choose to instantiate ParMeans with, ParMeansModule guarantees to produce an implementation for the instantiation of ParMeans.

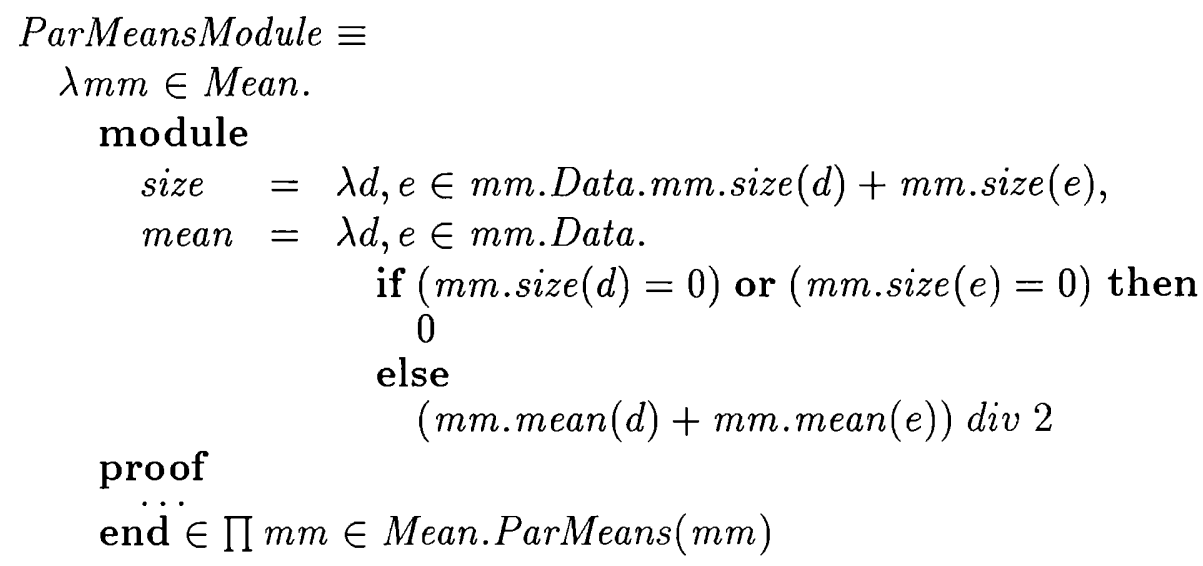

Figure 3.15: A parameterised version of MeansModule

As a separate point, note that ParMeans takes a module satisfying Mean as its parameter, not the specification Mean itself. The only reason for passing the specification 
Mean as a parameter is if we want to incorporate Mean into the body of ParMeans by using some specification operator. But that is not our intention here; we are only interested in using the components supplied by Mean. The example shows how the type membership relation between specifications and modules can be used to mix specifications and modules to make specifications.

\subsection{Overloading}

One feature of specifications and modules is that we allow different components within a signature to have the same name. We call this feature "overloading" of names; note that this use of the word "overloading" is different from its normal use where it means giving a symbol, or name, different meanings depending on the context in which the symbol is used. In practice, we make little deliberate use of overloading, but it can arise during program development, so we allow it. Potentially, overloading can cause ambiguities when it is not clear to which declaration the use of an overloaded name is bound. Additional scope rules are needed to resolve such ambiguities.

In a signature such as $y_{1} \in A_{1}, \ldots, y_{n} \in A_{n}$, each name $y_{i}(0 \leq i \leq n)$ is usually in scope in all types, $A_{i+1} \ldots A_{n}$, occurring to the right of $y_{i}$. But if some $y_{i}$ is overloaded, then each declaration of $y_{i}$ is only in scope from its point of declaration up to, but not beyond, the next declaration of the name $y_{i}$. Consequently, an overloaded name always binds to its most recent declaration. Similarly, occurrences of an overloaded component name in the restriction of a specification always bind to the last declaration of that name in the signature of the specification.

The following specification illustrates the scope rules for overloaded names:

$$
S=\text { Elements } x \in \mathbb{N}, y \in\{x\}_{\mathbb{N}}, x \in \mathbb{N}, z \in\{x\}_{\mathbb{N}} \text { Restrictions } x=_{\mathrm{N}} y \text { End }
$$

The name $x$ is overloaded in $S$. The type of $y$ is a singleton type containing the value of the first component $x$; in other words, $y$ is specified as being equal to $x$. The type of $z$ is also a singleton type $\{x\}_{N}$, but in this case $x$ is bound to the $x$ declared as the third component of $S$. The $x$ referred to by the restriction is also bound to the $x$ declared as the third component of the signature.

We also permit component names to be overloaded in the computational element of a module. In a computational element, any occurrence of an overloaded component name $p$, for example, always binds to the closest definition of $p$ that is in scope. For example, consider the following computational element:

$$
m n=\text { module } x=1, y=x, x=2, z=x \text { end } \in S
$$

Note that component name $x$ is overloaded in $m n$. The occurrence of $x$ in $y=x$ binds to $x=1$. But the occurrence of $x$ in $z=x$ binds to $x=2$ since $x=2$ is the closest definition of $x$ to $z=x$. 
If a component name $p$, for example, is overloaded in a computational element $m$, then $m . p$ is the value bound to the last definition of $p$ in $m$; in other words, m.p is the value bound to the definition of $p$ that is closest to the end of $m$. For example, if we consider computational element $m n$ above, then $m n . x=2$ since $x=2$ is the last definition of $x$ in $m n$. We can refer to component $x=1$ by temporarily renaming $x$ in $x=1$ to some unused component name $y$, for example, and then refer to $x=1$ as $m n . y$; renaming component names in computational elements is discussed in Chapter 6.

\subsection{An Observational Equality}

When we make a specification, we should try to ensure that it does not over-specify the system it is intended to specify. Over-specification may result in a specification that excludes acceptable implementations of a system. In other words, some modules may behave like acceptable implementations of a system, without actually satisfying its specification; we say such modules are observationally equivalent to implementations of the specification. One cause of over-specification in our specifications is the use of computational equality to equate expressions in the restrictions of our specifications. In this section, we show how loosening the equality relations used in specifications leads to looser specifications that admit observationally equivalent implementations previously excluded.

We proceed by example, considering the specification for sequences of naturals and showing why it fails to admit a particular module as an implementation. We then show how the equalities in the sequence specification may be loosened to admit the previously excluded implementation. Finally, we indicate how the task of loosening equalities can be generalised.

\subsubsection{The Problem}

Consider the specification SeqNat, given in Figure 3.16, which specifies sequences of naturals-SeqNat is produced by expanding the application of the generic specification Sequences to $\mathbb{N}$. SeqNat is a typical example of the specification of an abstract data type: it specifies a type, together with operations over the type. When we specify abstract data types we are usually interested in the so-called ultra-loose [56] interpretation of the semantics of the data type. However, SeqNat does not specify all the modules that an ultra-loose interpretation of SeqNat would allow. Consider an implementation of $S e q N a t$ where $S e q$ is implemented by the type $\operatorname{List}(\mathbb{N} \times \mathbb{B})$. Each integer element in a list is tagged (paired) with a boolean value; if the tag is true then 


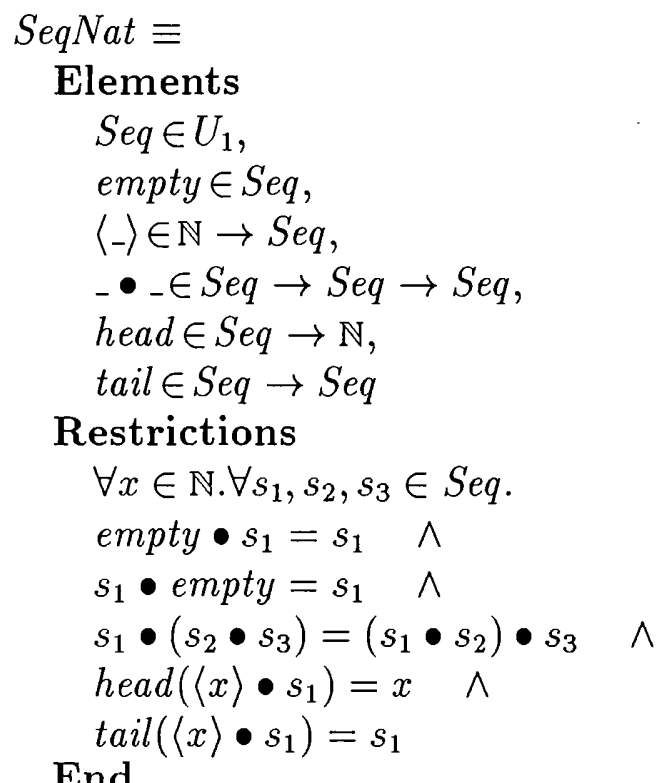

Figure 3.16: A Specification of Sequences of Naturals

the tagged integer is part of the sequence, and if the tag is false then the element is not part of the sequence. Consider the two sequences $s_{1}$ and $s_{1}$ :

$$
\begin{aligned}
& s_{1}=[(2, \text { false }),(4, \text { true }),(6, \text { true }),(8, \text { false })] \\
& s_{2}=[(4, \text { true }),(6, \text { true })]
\end{aligned}
$$

If we treat sequence as "black boxes" and only look inside them using head then $s_{1}$ and $s_{2}$ can be viewed as equivalent; for example, applying any sequence operation to $s_{1}$ and $s_{2}$, respectively, and then applying head will give equal results. Functions such as head which can look at the constituent parts of the values in a type are called "observers" of the type. Sequences $s_{1}$ and $s_{2}$ are said to be observationally equivalent with respect to the observers of $S e q$ in $S e q N a t$, and we write this as $s_{1} \cong_{S e q} s_{2}$. Removing elements from such sequences is simply a matter of tagging the elements with false: such an implementation is often used where explicitly removing elements from sequences is an expensive operation. By using $\operatorname{List}(\mathbb{N} \times \mathbb{B})$ to represent sequences, tail may be implemented as a function that marks the first occurrence of a true tag to false; and the head function returns the first element with a true tag. A complete implementation for sequences using $S e q=\operatorname{List}(\mathbb{N} \times \mathbb{B})$ is given by SeqImp2 in Figure 3.17. Note that in defining SeqImp2 we assume the existence of the functions $h d$ and $t l$ which are the usual head and tail functions, respectively, on list values.

Although SeqImp2 behaves as we would expect an implementation of sequences to behave, it does not satisfy SeqNat. In particular the equation,

$$
\forall x \in \mathbb{N} . \forall s \in \operatorname{Seq} . \operatorname{tail}(\langle x\rangle \bullet s)=_{\text {Seq }} s
$$




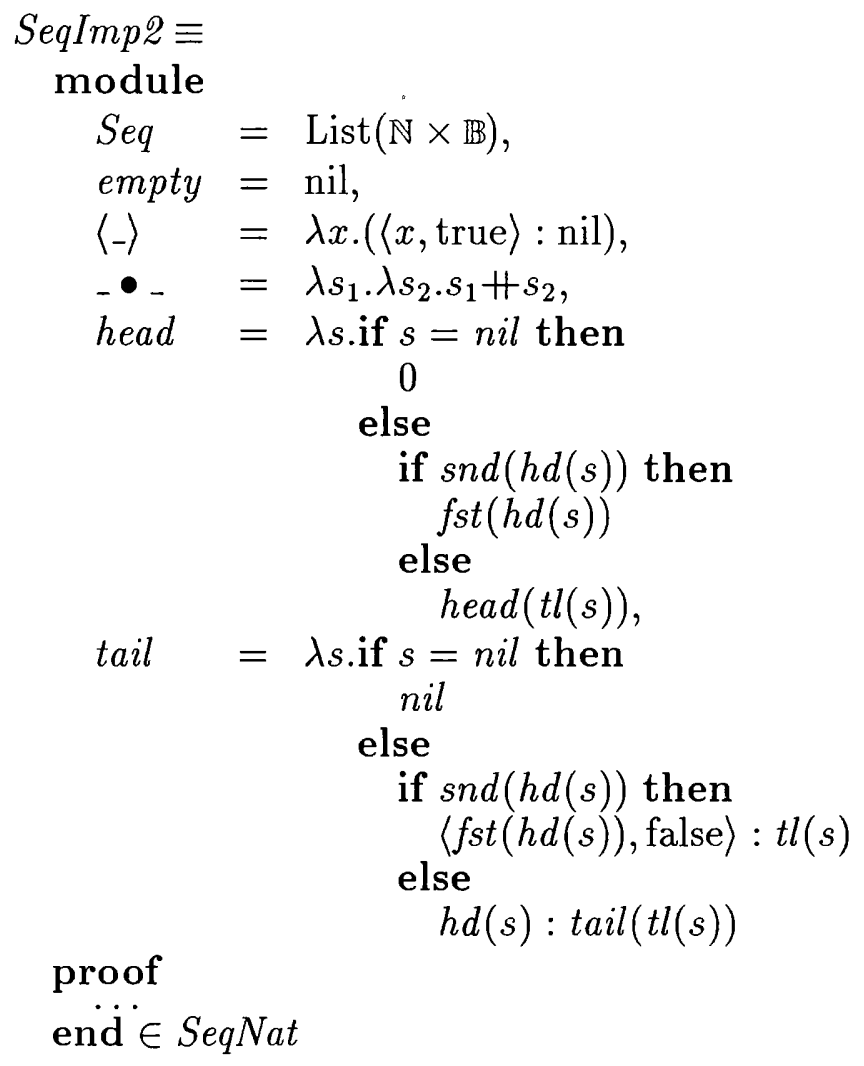

Figure 3.17: A loose implementation of SeqNat

does not hold. For example, given $x=1$ and $s=[(2$, true $),(3$, true $)]$ then:

$$
\begin{aligned}
& \operatorname{tail}(\langle x\rangle \bullet s) \\
& =\text { "defn }(-\bullet-) " \\
& \operatorname{tail}([(1, \text { true }),(2, \text { true }),(3, \text { true })]) \\
& =\text { "defn } \operatorname{tail} " \\
& \quad[(1, \text { false }),(2, \text { true }),(3, \text { true })] \\
& \neq \text { "list equality" } \\
& \quad[(2, \text { true }),(3, \text { true })] \\
& =\text { "given" }
\end{aligned}
$$

$S$

The reason for the inequality, above, is that we are comparing lists using the equality $={ }_{S e q}$ which is the built in computational equality $=_{\operatorname{List}(\mathbb{N} \times \mathbb{E})}$ on List $(\mathbb{N} \times \mathbb{B})$. Under this equality, two lists are equal iff they contain the same number of elements, and the corresponding elements are equal. However, from our intuitive understanding we recognise that $\operatorname{tail}(\langle x\rangle \bullet s)$ is observationally equivalent to $s$. (i.e. $\operatorname{tail}(\langle x\rangle \bullet s) \cong_{S e q} s$ ). In order to allow implementations such as SeqImp2 to satisfy SeqNat we must weaken the computational equality, $=_{S e q}$, to an observational equality $\cong_{S e q}$. We do this by adding $\cong_{S e q}$ to SeqNat. 


\subsubsection{A Solution}

The specification SeqNat2, in Figure 3.18, gives a loose specification of sequences, with an explanation following. We add an observational equality relation, $\cong_{S e q}$, as a new component to $S e q N a t$ and use it in place of the computational equality $=_{S e q}$. The type of $\cong_{S e q}$ is defined as $S e q \rightarrow S e q \rightarrow U_{1}$ : for any $s_{1}, s_{2} \in S e q, s_{1} \cong_{S e q} s_{2}$ is a type, and hence, a proposition. The equality $\cong_{S e q}$ is specified as a congruence w.r.t the operations defined by SeqNat. A formal definition of $\cong_{S e q}$ is given by Congruence $\left(\cong_{S e q}\right)$. Equations (1), (2) and (3) specify $\cong_{S e q}$ to be an equivalence. Axiom (4) states that if two sequences are observationally equivalent then the sequences obtained by applying tail to each of them are also observationally equivalent. Axiom (5) states that for two observationally equivalent sequences their head values are identical. Finally, axiom (6) states that if $s_{1}$ and $s_{2}$ are observationally equivalent, and if $s_{3}$ and $s_{4}$ are observationally equivalent, then $s_{1} \bullet s_{3}$ is observationally equivalent to $s_{2} \bullet s_{4}$. Note that the axiom head $\left(\langle x\rangle \bullet s_{1}\right)=_{N} x$ uses computational equality on naturals; computational equalities are always sufficient for comparing the values of primitive types such as $\mathbb{N}$, $\mathbb{B}$ etc.

Since SeqNat2 specifies the observational equality $\cong_{S e q}$ as part of its signature, modules satisfying SeqNat2 will contain an implementation of $\cong_{S e q}$ as a local component. For example, the loose implementation SeqImp2 can be made into an implementation of SeqNat2 by adding a suitable implementation of $\cong_{S e q}$ such as:

$$
-\cong_{S e q-}=\lambda s_{1} \cdot \lambda s_{2} \cdot\left[\operatorname{norm}\left(s_{1}\right)==_{L i s t(\mathrm{~N})} \operatorname{norm}\left(s_{2}\right)\right]
$$

here

$$
\begin{array}{ccc}
\text { norm } & \in \quad \text { List }(\mathbb{N} \times \mathbb{B}) \rightarrow \operatorname{List}(\mathbb{N}) \\
\text { norm }= & \lambda s . \text { if } s=\text { nil then } \\
& \text { nil } \\
\text { else } & \text { if } \operatorname{snd}(h d(s)) \text { then } \\
& \quad f s t(h d(s)): \text { norm }(t l(s)) \\
& \text { else } \\
& \text { norm }(t l(s))
\end{array}
$$

The function norm takes any sequence $s \in \operatorname{Seq}(\operatorname{Seq}=\operatorname{List}(\mathbb{N} \times \mathbb{B}))$ and transforms it into a sequence of natural numbers by removing the tags on the elements of $s$; elements with false tags are removed entirely. Function norm essentially normalises $S e q$ values to a canonical form. Hence, $\cong_{S e q}$ defines any two sequences $s_{1}, s_{2} \in S e q$ as observationally equivalent if their normalised forms are computationally equivalent under $\operatorname{List}(\mathbb{N})$ equality. 


\section{SeqNat2 $\equiv$}

\section{Elements}

$S e q \in U_{1}$,

-_ $_{\text {Seq }} \in \in S e q \rightarrow S e q \rightarrow U_{1}$, empty $\in S e q$,

$\langle-\rangle \in \mathbb{N} \rightarrow S e q$,

${ }_{-}{ }_{-} \in S e q \rightarrow S e q \rightarrow S e q$,

head $\in S e q \rightarrow \mathbb{N}$,

tail $\in S e q \rightarrow S e q$

\section{Restrictions}

$\forall x \in \mathbb{N} . \forall s_{1}, s_{2}, s_{3} \in$ Seq.

Congruence $\left(\cong_{\text {Seq }}\right) \wedge$

empty $\bullet s_{1} \cong_{S e q} s_{1} \wedge$

$s_{1} \bullet$ empty $\cong_{S e q} s_{1} \wedge$

$s_{1} \bullet\left(s_{2} \bullet s_{3}\right) \cong_{S e q}\left(s_{1} \bullet s_{2}\right) \bullet s_{3} \quad \wedge$

$\operatorname{head}\left(\langle x\rangle \bullet s_{1}\right)={ }_{\mathrm{N}} x \wedge$

\section{End}

$$
\operatorname{tail}\left(\langle x\rangle \bullet s_{1}\right) \cong_{S e q} s_{1}
$$

where

$$
\begin{aligned}
& \text { Congruence }\left(\cong_{S e q}\right) \equiv \\
& \forall s_{1}, s_{2}, s_{3}, s_{4} \in S e q \\
& s_{1} \cong{ }_{S e q} s_{1} \wedge \\
& s_{1} \cong{ }_{S e q} s_{2} \Rightarrow s_{2} \cong S_{S e q} s_{1} \wedge \\
& \left(s_{1} \cong_{S e q} s_{2} \wedge s_{2} \cong_{S e q} s_{3}\right) \Rightarrow s_{1} \cong_{S e q} s_{3} \wedge \\
& s_{1} \cong_{S e q} s_{2} \Rightarrow \operatorname{tail}\left(s_{1}\right) \cong_{S e q} \operatorname{tail}\left(s_{2}\right) \wedge \\
& s_{1} \cong_{S e q} s_{2} \Rightarrow \operatorname{head}\left(s_{1}\right)==_{\mathrm{N}} \operatorname{head}\left(s_{2}\right) \wedge \\
& \left(s_{1} \cong_{S e q} s_{2}\right) \wedge\left(s_{3} \cong_{S e q} s_{4}\right) \Rightarrow s_{1} \bullet s_{3} \cong_{S e q} s_{2} \bullet s_{4}
\end{aligned}
$$

Figure 3.18: A looser version of SeqNat 


\subsubsection{A Generalisation of Observational Equivalence}

The choice of whether to define a congruence between the values of a type is a specification design decision. Not every abstract data type we specify requires a congruence. If we specify ADT's whose values are to be treated as "black boxes"such as stacks, queues, containers etc - then we should specify a congruences between their values. On the other hand, if we intend to specify operations that compare the values of an $\mathrm{ADT}$ for equality then we should not use congruences. The penalty for using congruences is that the specification becomes more complicated. The penalty for not using congruences is that we may disallow implementations that might well have been suitable for our needs. It could be left up to an implementor to decide whether a congruence is used or not, but adding a congruence "weakens" a specification, and will allow implementations that may not have been considered by the specifier. This may lead to implementations that do not satisfy the original specification, although they will be observationally equivalent to implementations of the original specification.

If we specify an abstract data type - $T$, say - and decide to use congruence for equality between $T$-valued expressions, then we can generate the axioms that specify the congruence in a systematic manner. To define a congruence $\cong_{T}-\in T \rightarrow T \rightarrow U_{1}$ over type $\mathrm{T}, \cong_{T}$ must first be an equivalence:

$$
\begin{array}{ll}
\forall t_{1}, t_{2}, t_{3} \in T . & \\
t_{1} \cong_{T} t_{1} \wedge & \text { (reflexive) } \\
t_{1} \cong_{T} t_{2} \Rightarrow t_{2} \cong_{T} t_{1} \wedge & \text { (symmetric) } \\
\left(t_{1} \cong_{T} t_{2} \wedge t_{2} \cong_{T} t_{3}\right) \Rightarrow t_{1} \cong_{T} t_{3} & \text { (transitive) }
\end{array}
$$

When we defined the congruence on sequence values in Figure 3.18, it was shown that the operations in SeqNat also generated axioms about the congruence. In general, if the specification of $T$ specifies $m$ operations $f_{j}(0 \leq j \leq m)$ then each operations generates an axiom. For each operation $f_{j} \in T_{1} \rightarrow T_{2} \rightarrow \ldots \rightarrow T_{n}$ the following axiom is generated:

$$
\begin{aligned}
& \forall t_{1}, t_{1}^{\prime} \in T_{1} \ldots \forall t_{n-1}, t_{n-1}^{\prime} \in T_{n-1} . \\
& \quad\left(t_{1} \equiv_{T_{1}} t_{1}^{\prime}\right) \wedge \ldots \wedge\left(t_{n-1} \equiv_{T_{n-1}} t_{n-1}^{\prime}\right) \Rightarrow f_{j}\left(t_{1}, \ldots, t_{n-1}\right) \equiv_{T_{n}} f_{j}\left(t_{1}^{\prime}, \ldots, t_{n-1}^{\prime}\right)
\end{aligned}
$$

where for each type $T_{i}(1 \leq i \leq n)$, if $T_{i}$ is a primitive built-in type (such as $\mathbb{N}, \mathbb{B}$ etc) then $\equiv_{T_{i}}$ is the usual computational equality $=_{T_{i}}$; and if $T_{i}=T$ then $\equiv_{T_{i}}$ is the equivalence $\cong_{T}$.

All the axioms described by the above "prescription" are sufficient to specify a congruence on type $T$, assuming only operations $f_{j}(0 \leq j \leq m)$ are used on $T$-values. If we decide to add new operations on $T$-values to the specification, then we must also add new axioms about the congruence: the new operations generate axioms in the same way each operation $f_{j}$ did above. 


\subsection{Conclusion and Summary}

The examples of specifications and modules given above do not describe all the features of our specification language, but only serve to illustrate the style of specifications and modules we use. Wirsing and Broy [56] have used congruence relations to specify observational equivalences within algebraic specifications; they define a simple algebraic specification language that equips every sort in a specification with a congruence relation instead of the usual computational equality. Although our typetheoretic approach to specifications is similar in many ways to algebraic specifications, it is important to stress that many of the specifications presented in this chapter are different from algebraic specifications; the examples of model-oriented specifications, and specifications being used as data-types, are intended to convince the reader of this fact.

We have not discussed the semantics of specifications in this chapter. However, if we are do any formal reasoning with specifications then we need a formal semantics, and this is discussed in Chapter 4.

In summary, we have seen that specifications and modules may have local components that can be used to factor the specification and implementation of complicated components. We also show how the built-in types and operations of the type theory may be used to make specifications and modules. Specifications can also be used as normal data types; this allows us to nest specifications and modules, and also to use modules as records. 


\section{Chapter 4}

\section{The Semantics of Specifications}

\subsection{Introduction}

It is important that specification and implementation languages should have a formal semantics. Firstly, a formal semantics can resolve possible ambiguities about the meaning of specifications. Secondly, the semantics can be used to deduce the properties of a program from its specification. Thirdly, the semantics can be used to justify laws for refining and implementing specifications. In this chapter, we define the semantics of our canonical specifications and modules in Martin-Löf's type theory.

The are several reasons why we choose to define the semantics of specifications and modules in type theory. Firstly, a type-theoretic semantics for specifications and modules is simple because the formal language of type theory can be used as a programming language, a specification language and a programming logic. Consequently, the semantics of specifications and modules can be given in the single framework of type theory; no other theory is required. Secondly, features of the type theory such as dependent sum types, and propositions, are particularly suited to specifying modules: for example, the dependent sum type readily captures the inter-dependencies between components in a module. Thirdly, type theory provides a simple definition of a module satisfying a specification: recall that a specification is a type, and that the values of the type are modules satisfying the specification.

Our semantics for specifications and modules is based on the approach of Nordström, Petersson and Smith $[43,44]$ (henceforth known as the NPS approach) to specifying modules in Martin-Löf's Type Theory. The NPS approach has influenced other work on specifying modules in type theory, most notably the deliverables approach advocated by Burstall et al [6] and Luo [27]. Both approaches use dependent sum types, in conjunction with the principle of propositions as types, to specify modules in type theory. 
We will give a semantics to our specifications and modules by translating them into terms in MLT. This chapter defines the terms of MLT that we use as translations for specifications and modules. A formal definition of a mapping from the syntax of specifications and modules to their translations in MLT will be given in Chapter 5 . This split in the presentation of the semantics is purely for presentation. The terms used as translations for specifications and modules can be understood without a formal definition of the translation mapping; its definition in this chapter would simply obscure the definitions of those terms.

In the NPS and deliverables approaches, specifications and modules are written directly as terms in MLT, but we chose not to do this as the translations of our specifications and modules are complicated terms; it is easier for a programmer to write them in their un-translated form. The translations are complicated by the need to give a semantics to component names and local components within specifications and modules. Neither the NPS nor deliverables approaches address those issues. We need to give a semantics to component names and local components to define specification operations such as renaming and hiding, and module operations such as dot notation. However, giving a semantics to names is problematic: the translations of specifications and modules must distinguish between the use of names as bound variables to describe dependencies between components, and the use of names to refer to components in specifications and modules.

One feature of our semantics is that we do not add any new types to MLT. The translations of specifications and modules are all existing terms in MLT, and can be seen as constituting a meta-theory sitting on top of MLT.

\subsection{A review of semantics}

We begin by reviewing the NPS and deliverables approaches. In those approaches, a programmer writes specifications and modules as terms in type theory: specifications denote dependent sum types and modules denote nested pairs. Therefore, it is unnecessary to translate specifications and modules into terms in the type theory. Neither approach includes component names or local components in specifications and modules. The NPS and deliverables approaches differ in their treatment of restrictions in specifications, and witnesses in modules; we will illustrate these differences below.

We review the NPS and deliverables approaches by example. First, we give an example specification and module written in our specification and implementation language, respectively. Then we show how our example specification and module would be written in the NPS and deliverables approaches and say what the new specifications and modules denote. Finally, we give a critique of both approaches. 


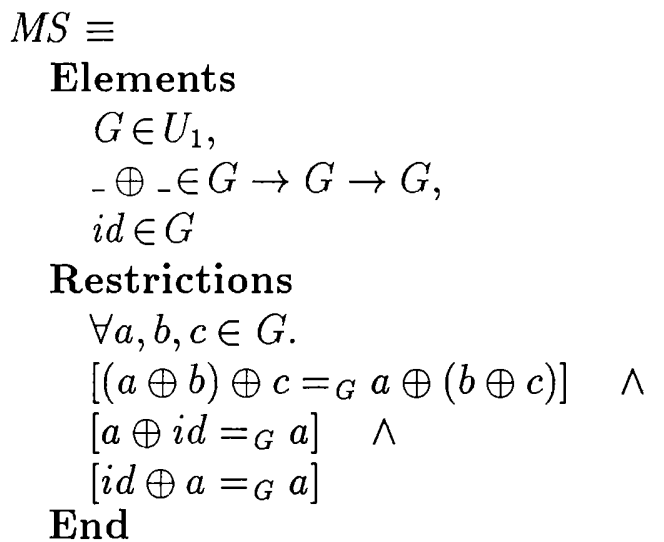

Figure 4.1: The specification of monoids

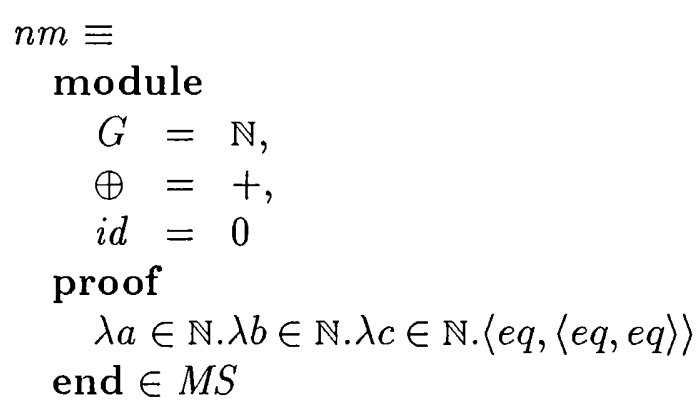

Figure 4.2: A module describing a monoid

\subsubsection{A running example-Monoids}

We consider the specification of monoids which is given in Figure 4.1 with explanation following. In mathematics a monoid is an algebra consisting of a carrier set, a binary operation and an identity value; all of which satisfy certain relationships. Component $G$ is a type representing the carrier of the monoid; $\oplus$ is an associative infix binary operator with type $G \rightarrow G \rightarrow G$; and $i d$ is a two-sided identity for $\oplus$. A familiar monoid is the naturals $(\mathbb{N})$ with the integer addition operator $(+)$ and identity 0 ; in mathematics such a monoid is usually packaged as the tuple $\langle\mathbb{N},+, 0\rangle$. Other examples of monoids include $\langle\operatorname{List}(\mathbb{N}), \#, n i l\rangle$ where $\#$ is list concatenation and $n i l$ is the empty list; and $\langle$ Bool,$\wedge$, true $\rangle$ where $\wedge$ is the logical-and operator. We can describe monoids by modules in our implementation language; for example, the module $n m$, given in Figure 4.2, describes the monoid $\langle\mathbb{N},+, 0\rangle$. Note that $n m$ includes a witness; the purpose of the witness will become clear later.

\subsubsection{Specifications in the NPS approach}

$M S_{1}$, in Figure 4.3, is a specification of monoids in the NPS approach, with explanation following. There is a strong syntactic resemblance between $M S_{1}$ and our specifi- 


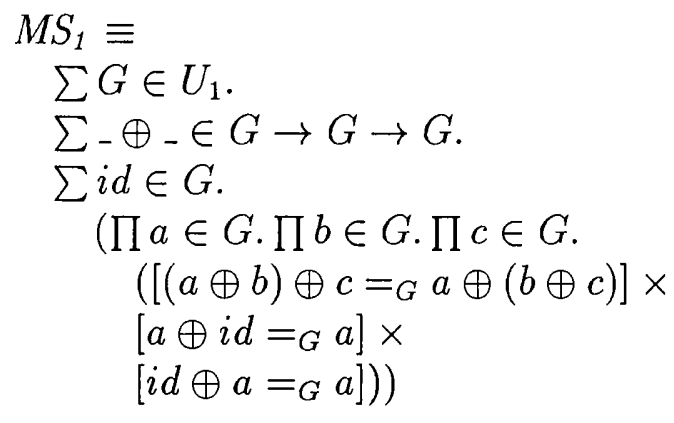

Figure 4.3: A Specification of monoids in the NPS approach

cation $M S$. However, specifications in the NPS approach are terms in type theory that denote dependent types; $M S_{1}$ is what a programmer would write as a specification for monoids. Dependent product types can express the type dependencies that may exist between components in a module; for example, $M S_{1}$ expresses the fact that the types of the second $(\oplus)$ and third $(i d)$ components in a monoid are dependent on the value of the first component $(G)$. The names $G, \oplus$ and $i d$ are only bound variables. Dependent product types alone cannot specify the behaviour of components in specifications. That is done by adding a final field which is a proposition specifying the behaviour of the preceding components. The proposition specifying the properties of $G, \oplus$ and $i d$ in $M S_{1}$ is $A x\left(M S_{1}\right)$, where

$$
\begin{aligned}
A x\left(M S_{1}\right)=( & \left(\prod a \in G . \prod b \in G . \prod c \in G .\right. \\
& \left(\left[(a \oplus b) \oplus c={ }_{G} a \oplus(b \oplus c)\right] \times\right. \\
& {\left[a \oplus i d==_{G} a\right] \times } \\
& {\left.\left.\left[i d \oplus a={ }_{G} a\right]\right)\right) }
\end{aligned}
$$

Using the isomorphism between types and propositions, it can easily be shown that $A x\left(M S_{1}\right)$ corresponds to the classical proposition:

$$
\forall a, b, c \in G .((a \oplus b) \oplus c=a \oplus(b \oplus c) \wedge a \oplus i d=a \wedge i d \oplus a=a)
$$

which is identical to the restriction in our specification $M S$.

\subsubsection{Modules in the NPS approach}

In the NPS approach, modules are terms in type theory, and these terms denote pairs. The pairs are members of the types that specifications denote. A module $m$ is said to "satisfy" a specification $S P$ if $m \in S P$. For example, $n m_{1}$, below, is a module that describes a monoid, and $n m_{1}$ satisfies $M S_{1}$ :

$$
n m_{1}=\langle\mathbb{N},\langle+,\langle 0, \lambda a \in \mathbb{N} . \lambda b \in \mathbb{N} . \lambda c \in \mathbb{N} .\langle e q,\langle e q, e q\rangle\rangle\rangle\rangle\rangle \in M S_{1}
$$

Note that $n m_{1}$ is constructed from nested pairs since $M S_{1}$ is a nested dependent sum type. The module $n m_{1}$ describes the same monoid as the module $n m$ which was given in Figure 4.2. In the NPS approach, the components in modules are not 
named, and modules do not allow local components. We can refer to the components in $n m_{1}$ by compositions of $f s t$ and snd. The lack of names means that such modules cannot express dependencies between their components. The final value in a module is a witness. For example, the final value in $n m_{1}$,

$$
\lambda a \in \mathbb{N} . \lambda b \in \mathbb{N} . \lambda c \in \mathbb{N} .\langle e q,\langle e q, e q\rangle\rangle,
$$

is a witness that is a member of the proposition $A x\left(M S_{1}\right)$, and is equal to the witness of the module $n m$.

To see how the components in modules that satisfy $M S_{1}$ obey the proposition $A x\left(M S_{1}\right)$, consider any module $\langle G,\langle\oplus,\langle i d, r\rangle\rangle\rangle \in M S_{1}$. The extra component $r$ is a witness and belongs to the type $A x\left(M S_{1}\right)$. Since we know that $r \in A x\left(M S_{1}\right)$ we know that for the particular values $G, \oplus$ and $i d$ in $\langle G,\langle\oplus,\langle i d, r\rangle\rangle\rangle$ the type $A x\left(M S_{1}\right)$ is inhabited, i.e it is true. Therefore, we can conclude that the corresponding classical proposition is true for $G, \oplus$ and $I d$; the value $r$ is a witness that proves that the proposition is true. The actual value of $r$ is not important.

\subsubsection{Specifications in the deliverables approach}

In the deliverables approach, specifications are terms in type theory that denote dependent product types. $M S_{2}$, in Figure 4.4, is a specification of monoids, in the deliverables approach. $M S_{2}$ is a straight translation of $M S_{1}$. In the deliverables approach, the left field of a specification is called the structure type of the specification. The structure type of $M S_{2}$ is a dependent product type $S$, where

$$
S=\sum G \in U_{1} \cdot \sum_{-} \oplus_{-} \in G \rightarrow G \rightarrow G . G
$$

The fields in $S$ are the data-types of the components included in $M S_{2}$. The right field of $M S_{2}$ is a proposition that specifies axioms about the components included in $S$; we name this proposition $A x\left(M S_{2}\right)$. The main difference between specifications in the NPS and deliverables approaches is that in the deliverables approach axioms are defined independently of the bound component names given in the structure type. For example, note that the bound names $G$ and $\oplus$, in $S$, are not in scope in $A x\left(M S_{2}\right)$. $A x\left(M S_{2}\right)$ is defined in terms of the single bound variable $m \in S$, and refers to the components of $m$ by compositions of $f s t$ and $s n d$. We abbreviate the use of projection functions by $m_{1}, m_{2}$ and $m_{3} ; m_{1}$ is the carrier of a monoid; $m_{2}$ is the associative binary operator; and $m_{3}$ is the two sided identity value.

The main advantage of the deliverables approach-over the NPS approach-is that specifications can easily be factored into their "computational contents" (given by the structure type) and their axiomatic requirements. In the NPS approach, the axioms are nested deep within a $\sum$-type and this makes factorisation harder. Factoring a specification has several advantages. Firstly, it simplifies the definition of operations 


$$
\begin{aligned}
& M S_{2} \equiv \\
& \quad \sum m \in\left(\begin{array}{l}
\sum_{-} G \in U_{1} . \\
G
\end{array} \oplus_{-} \in G \rightarrow G \rightarrow G .\right) \cdot\left(\begin{array}{l}
\forall a, b, c \in m_{1} . \\
\left(a\left(m_{2}\right) b\right)\left(m_{2}\right) c=a\left(m_{2}\right)\left(b\left(m_{2}\right) c\right) \wedge \\
a\left(m_{2}\right)\left(m_{3}\right)=a \wedge \\
\left(m_{3}\right)\left(m_{2}\right) a=a
\end{array}\right)
\end{aligned}
$$

here $m_{1}, m_{2}$ and $m_{3}$ are abbreviations defined by:

$$
\begin{aligned}
& m_{1}=f s t(m) ; \\
& m_{2}=f s t(\operatorname{snd}(m)) ; \text { and } \\
& m_{3}=\operatorname{snd}(\operatorname{snd}(m))
\end{aligned}
$$

Figure 4.4: A specification for monoids in the deliverables approach

on specifications as it allows definitions to be factored into two parts: one part defines the operation over the structure type, and the other part defines the operation over the axioms. Secondly, it makes it easier to separate redundant witnesses from modules satisfying such specifications; this is described in the next section.

\subsubsection{Modules in the deliverables approach}

Modules in the deliverables approach are similar to modules in the NPS approach as they are also terms in type theory that denote nested pairs. For example, $n m_{2}$, below, is a module in the deliverables approach, and it satisfies $M S_{2}$ :

$$
n m_{2}=\langle\langle\mathbb{N},\langle+, 0\rangle\rangle, \lambda a \in \mathbb{N} . \lambda b \in \mathbb{N} . \lambda c \in \mathbb{N} .\langle e q,\langle e q, e q\rangle\rangle\rangle \in M S_{2}
$$

The only difference between modules in the NPS and deliverables approaches is the way in which components are nested. In the deliverables approach, the witness in a module - $m$, say -is nested so that it is $\operatorname{snd}(m)$, and the other "computational" components are, collectively, $f s t(m)$.

As with specifications, there are some advantages to the ease with which a deliverables module can be factored into its witness and computational components. Firstly, it simplifies proofs concerning modules: most constructive proofs concerning modules need to refer to the witness within a module, and the easier this is, the clearer the proof. Secondly, it simplifies the definition of many operations on modules by allowing the definition to be factored into an operation over the computational components of a module, and an operation over the witness.

\subsubsection{A Critique}

The NPS and deliverables approaches illustrate that, in principle, type theory can be used as the basis for the semantics of specifications and modules. Our main criticism of both the NPS and deliverables approaches is the omission of a name-space for 
components within specifications and modules. The failure to address the name-space issue impedes the use of both approaches as the basis for a full-blown specification and implementation language for modules. Both approaches fail to recognise that component names play a dual role: on the one hand they specify dependencies between components, and on the other hand they name components within specifications and modules, so that client specifications and modules can refer to components by name. Both the NPS and deliverables approach only recognise the role of component names in specifications as that of specifying dependencies.

At first sight, $M S_{1}$ does appear to introduce the names $G, \oplus$ and $i d$ for the components it specifies. However, $G, \oplus$ and $i d$ are only bound variables within $M S_{1}$, and so can be changed without changing the meaning of the specification. The lack of component names in the deliverables style means that a programmer must use $f s t$ and snd to write the proposition that specifies the behaviour of the components in a specification. Clearly, this is undesirable as it complicates the specification, and makes references to components position dependent. The types that $M S_{1}$ and $M S_{2}$ denote are-type theoretically-isomorphic, but $M S_{2}$ is more complicated to write than $M S_{1}$. Nevertheless, the ease with which specifications and modules can be factored in the deliverables approach does make formal reasoning about specifications and modules easier than in the NPS approach.

Ideally, we would like a fusion of the NPS and deliverables approach in which the axioms can be defined using component names, but where specifications and modules can still be factored easily. In addition, we would like to add component names and local components to specifications and modules. The fusion of the two approaches, together with the additions described here, form the basis for the semantics of our specifications and modules.

\subsection{An Introduction to Our Semantics}

In this section, we give an introduction to our semantics of specifications and modules. Our semantics are given by translating specifications and modules into terms in Martin-Löf's Type Theory (MLT). The translation of a specification is a term in MLT that denotes a type. This type is a dependent product type whose left component is a translation of the signature of the specification, and whose right component is a translation of the restriction of the specification. It follows that the translations of signatures and restrictions are also terms in MLT that denote types. The translation of a module is a term in MLT that denotes a pair. The left and right components of this pair are the translations of the computational element and witness of the module, respectively. 
To distinguish between specifications as they are written, and their translation, we write the translation of a specification- $S P$, say-as $\llbracket S P \rrbracket$. We also write "specification in MLT" to mean a term in MLT obtained by translating a specification. Similarly, we write the translation of a signature- $S$, say -as $\llbracket S \rrbracket$, and write "signature in MLT" to mean a term in MLT obtained by translating a signature; and so on for modules, computational elements, restrictions and witnesses. In addition, we sometimes write "specification as a type" and "signature as a type" to mean "specification in MLT" and "signature in MLT", respectively.

We will require that members of specifications in MLT be modules in MLT. So the choice of translations for specifications also determines the translation of modules. A module $m$ is said to "satisfy" a specification $S P$ if $\llbracket m \rrbracket \in \llbracket S P \rrbracket$. Moreover, if $\llbracket m \rrbracket \in \llbracket S P \rrbracket$ then the computational element of $\llbracket m \rrbracket$ will be a member of the signature of $\llbracket S P \rrbracket$, and the witness of $\llbracket m \rrbracket$ will be a member of the restriction of $\llbracket S P \rrbracket$.

The main technical problem in defining translations for specifications concerns translations for signatures. The translation of a signature must describe the type of each component in the signature, and the domain of the signature, and it must distinguish between local and visible names. As we require the members of signatures in MLT to be computational elements in MLT, the choice of translations for signatures also determines the translations of computational elements. Computational elements in MLT will be treated like records, and we will again refer to their components using a Pascal style dot notation. Further discussion about the translations of signatures and computational elements is left until Sections 4.4 and 4.6, respectively.

\subsubsection{An Example Specification in MLT}

$$
\begin{aligned}
& \llbracket M S \rrbracket \equiv \\
& \sum m \in \llbracket G \in U_{i}, \oplus \in G \rightarrow G \rightarrow G, i d \in G \rrbracket . \\
& \forall a, b, c \in m . G \text {. } \\
& (a m . \oplus b) m . \oplus c=a m . \oplus(b m . \oplus c) \wedge \\
& a m . \oplus m . i d=a \wedge \\
& \text { m.id } m . \oplus a=a
\end{aligned}
$$

Figure 4.5: The translation of specification $M S$

Figure 4.5 gives the translation of specification $M S$ (as given in Figure 4.1). The expression $\llbracket G \in U_{i}, \oplus \in G \rightarrow G \rightarrow G, i d \in G \rrbracket$ denotes the translation of the signature of $M S$. The structure of $\llbracket M S \rrbracket$ is similar to that of a deliverables' specification. But, unlike the deliverables approach, the restriction of $\llbracket M S \rrbracket$ refers to the components in the computational element, $m$, by dot notation instead of $f s t$ and $s n d$. For example, the restriction of $\llbracket M S \rrbracket$ refers to component $G$ as m.G. Unlike dot notation on modules, dot notation on computational elements can refer to local components. 


\subsubsection{An Example Module in MLT}

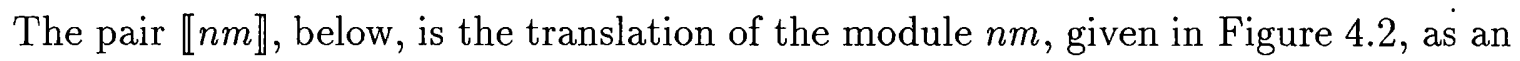
implementation of $M S$ :

$\llbracket n m \rrbracket=\langle\llbracket$ module $G=\mathbb{N}, \oplus=+, I d=0$ end $\rrbracket, \lambda a, b, c \in \mathbb{N} .\langle e q,\langle e q, e q\rangle\rangle\rangle$

The left component of $\llbracket n m \rrbracket$ denotes the translation of the computational element of $n m$. The right component of $\llbracket n m \rrbracket$ is the translation of the witness of $n m$. Note that the witness of $n m$ and its translation are syntactically identical; this is because the witness of $n m$ is already a term in MLT. $\llbracket n m \rrbracket$ is a member of type $\llbracket M S \rrbracket$, so $n m$ satisfies $M S$. Furthermore, the computational element of $\llbracket n m \rrbracket$ is a member of the signature of $\llbracket M S \rrbracket$, and the witness of $\llbracket n m \rrbracket$ is a member of the restriction of $\llbracket M S \rrbracket$.

Remark Section 4.8 contains a detailed example of the translation of a specification and module, and it may help the reader to refer to Section 4.8 when reading the formal semantics of specifications and modules which we give in the following sections.

\subsection{Signatures in MLT}

In this section we consider the translation of signatures. The translation of a signature is a term in MLT that denotes a product type. The left component of this type is just the domain of the signature packaged as a singleton type. The right component is called the "loose signature" of the signature. A loose signature is similar to the $\sum$-types used as specifications by the NPS and deliverables approaches, but where the restriction is omitted and where names are just treated as bound variables. We will give the domain of a signature a formal meaning as a term in MLT that denotes a list of tagged names for components in a signature; the tags indicate whether the name is local or visible. The word "domain" is overloaded as we will also use it to mean the domain packaged as a singleton type. In general, when we refer to the domain of a signature we will mean a list of tagged names, and if we use domain to mean a type then this will be clear from the context; if there is potentially any doubt about the use of domain to mean a type, we will write "domain as a type".

\subsubsection{An Illustration}

We illustrate the translation of signatures by considering the translation of:

$$
S=G \in U_{1}, \oplus_{-} \in G \rightarrow G \rightarrow G, i d \in G .
$$

The translation of $S$, written $\llbracket S \rrbracket$, is given below, with an explanation following:

$$
\left\{\left[\operatorname{inl}\left({ }^{\prime} G \text { ' }\right), \operatorname{inl}\left({ }^{\prime} \oplus \text { ') }, \operatorname{inl}\left({ }^{\prime} i d^{\prime}\right)\right]\right\}_{\text {List }(\mathbb{S}+\mathbb{S})} \times\left(\sum x \in U_{1} \cdot \sum y \in x \rightarrow x \rightarrow x . \sum z \in x . T\right)\right.
$$


The singleton type to the left of the product $(x)$ is the domain of $\llbracket S \rrbracket$, and the $\sum$-type on the right is the loose signature of $\llbracket S \rrbracket$. The names $x, y, z$ used in the loose signature are just bound variables that express the dependencies between components. Note that the loose signature is terminated by the unit type $T ; T$ is needed for technical reasons which we will explain later. The domain, as a type, is a singleton type containing the domain as a list. We define the domain as a singleton type since all computational elements meeting a signature have the same domain (list of names). The terms ' $G$ ', ' $\oplus$ ' and ' $i d$ ' are the translations of the names of the components in $S$ and have type $\mathbb{S}$. The names in the domain are tagged so that we can distinguish between local and visible components: visible component names are tagged with inl, and local names are tagged with inr. Tagged names have type $(\mathbb{S}+\mathbb{S})$. The names in the domain occur in the same order as they appear in signatures. The special case of the empty signature $(\Phi)$ is translated to the type $\{\text { nil }\}_{\text {List }(\mathbb{\$}+\mathbb{S})} \times T$ : the loose signature is the unit type $T$, and the domain is the empty list.

\subsubsection{The Signature Judgement}

In the following, we give the formal definition of a judgement sig, such that $S$ sig holds whenever $S$ is a term in MLT that is the translation of some signature. The judgement sig is not intended as a new judgement for the type theory, but is a metajudgement defined in terms of the existing judgements. Consequently, signatures do not have a formal standing within the type theory. For example, there are no new formation, elimination or introduction rules for signatures or their values. We use \{\} and $\sum$-formation, -introduction, and -elimination rules to construct and reason about signatures as types. The advantage of this approach is that we avoid extending the type theory, with the consequent obligation to prove that the extension is consistent with the existing theory.

To help define the judgement sig, we define a judgement lsg, such that $P$ lsg holds if $P$ is a well-formed loose signature; in other words, $P$ lsg holds if $P$ is a nested $\sum$-type terminated by $T$, or $P$ is just $T$.

\section{Definition 4.1 (Well-Formed Loose Signature)}

The judgement lsg is defined inductively as follows:

$$
\begin{aligned}
& T \text { lsg } \\
& \sum x \in A . B(x) \text { lsg iff } A \text { type and } \llbracket[x \in A \triangleright B(x) \text { lsg }] \\
& {[x \in A \triangleright T \text { lsg }] \text { iff } A \text { type }}
\end{aligned}
$$

Fact 4.1 Given a type term $P$, if $P$ lsg then $P$ type.

The type of a domain —as a list—is $\operatorname{List}(\mathbb{S}+\mathbb{S})$. We introduce the symbol $\mathbb{D}$ to denote the type $\operatorname{List}(\mathbb{S}+\mathbb{S})$. 
Notation $\mathbb{D}=\operatorname{List}(\mathbb{S}+\mathbb{S})$

Using the judgement lsg, we give the formal definition of a well-formed signature in MLT as the product of a domain (as type) and a loose signature:

\section{Definition 4.2 (Well-Formed Signature in MLT)}

$\{l\}_{\mathbb{D}} \times S$ sig iff $S$ lsg and $l \in \mathbb{D}$

Fact 4.2 Given a type term $S$, if $S$ sig then $S$ type.

Note, the definition of judgement sig does not require the length of the domain, $l$, to be equal to the number of components included in the loose signature $S$. In practice, the number of components in $l$ and $S$ are the same, but as we shall see later, we can define all the operations we will need without making use of this fact. The definition of sig does not prevent duplicate names appearing in a domain, and indeed duplicate names occur in the domain of a signature whenever we translate a signature that overloads the names of some of its components.

Loose signatures are terminated by the unit type $T$ in order to disambiguate between potentially ambiguous loose signatures. We illustrate the problem by considering the following signatures:

$$
\begin{aligned}
& S_{1}=y \in\left(\sum x \in P . Q(x)\right) \\
& S_{2}=y \in P, z \in Q
\end{aligned}
$$

$S_{1}$ includes a single component $y$ that is a pair, and $S_{2}$ includes two components $y$ and $z$ of type $P$ and $Q(y)$, respectively. The loose signatures of $S_{1}$ and $S_{2}$ are $L S_{1}$ and $L S_{2}$, respectively, where:

$$
\begin{aligned}
& L S_{1}=\sum y \in\left(\sum x \in P \cdot Q(x)\right) . T \\
& L S_{2}=\sum y \in P . \sum z \in Q . T
\end{aligned}
$$

If we do not terminate loose signatures with the unit type then the loose signature of $S_{1}$ and $S_{2}$ is:

$$
L S=\sum x \in P \cdot Q(x)
$$

$L S$ is ambiguous: $L S$ could be interpreted as a loose signature including two components of type $\mathrm{P}$ and $\mathrm{Q}$, respectively, or a loose signature including a single component that is a pair. Without a terminating unit type, both interpretations of $L S$ seem reasonable. This ambiguity is undesirable since $S_{1}$ and $S_{2}$ are different signatures. Such an ambiguity arises in all signatures whose final component is a pair, and can always be solved by using the unit type to mark the end of a loose signature.

\subsubsection{Some Operations on Signatures}

In order to reason about signatures in MLT, we define two functions, Dom and Lsg, which both take a signature in MLT, and return its domain and loose signature, 
respectively. The definitions of Dom and $L s g$ are given by definitions 4.3 and 4.4 , respectively, and an explanatory remark follows the definitions.

\section{Definition 4.3 (Dom)}

Given $\{l\}_{\mathbb{D}} \times S$ sig, and any $P$ type ( $P$ not a signature) then $D o m \in U_{1} \rightarrow \mathbb{D}$ is defined as follows:

$$
\begin{aligned}
& \operatorname{Dom}\left(\{l\}_{\mathbb{D}} \times S\right)=l \\
& \operatorname{Dom}(P) \quad=\text { nil }
\end{aligned}
$$

\section{Definition 4.4 ( $L s g)$}

Given $\{l\}_{\mathbb{D}} \times S$ sig, and any $P$ type ( $P$ not a signature) then $L s g \in U_{1} \rightarrow U_{1}$ is defined as follows:

$$
\begin{array}{ll}
\operatorname{Lsg}\left(\{l\}_{\mathbb{D}} \times S\right) & =S \\
\operatorname{Lsg}(P) & =P
\end{array}
$$

Remark For the sake of presentation, Dom and Lsg are given in a clausal form, although, strictly speaking, they should be defined using the urec elimination operator on types. Note that the functions Dom and $L s g$ are defined over all types, not just signatures, since operations on types must be total in MLT. Both definitions, have two clauses, one for signatures and one for non-signatures, and these cover all possible arguments to Dom and $L s g$. In practice, we are only interested in applying Dom and $L s g$ to signatures in MLT, and the definition of Dom and $L s g$ over non-signatures is not important.

Fact 4.3 If $S$ sig then $\operatorname{Lsg}(S)$ lsg.

\subsubsection{Scope within Signatures}

This section describes how signatures in MLT incorporate scope. The scope of a name in a signature is the scope of the bound variable it is translated to in the loose signature. Hence, the scope of names is enforced by the scope rules for bound variables in quantified terms of the type theory--we do not need to impose any extra rules, which is one of the advantages of our choice of translations for signatures. The scope rules for quantified expressions in MLT are summarised by saying that the scope of a bound variable extends from its declaration to the next unmatched parenthesis. But, if a variable is declared twice, such as $x$ in $\sum x \in P . \sum x \in Q \cdot R(x)$, then free occurrences of the variable bind to the closest declaration of the variable; so, $x$ in $R(x)$ binds to $x \in Q$. Full details about the scope of bound variables in MLT are given in [44]. 
We illustrate the incorporation of scope within signatures with an example. Consider the signature:

$$
N S=p \in P_{0}, q \in\left(p \in P_{1}(p), r \in R(p)\right), p \in P_{2}(p, q), s \in S(p, q)
$$

$N S$ overloads the name $p$, and moreover, $p$ occurs a further time in the type of $q$. The loose signature of $N S$ is given below:

$$
\sum p \in P_{0} . \sum q \in\left(\sum p \in P_{1}(p) . \sum r \in R(p) . T\right) . \sum p \in P_{2}(p, q) . \sum s \in S(p, q) . T
$$

Let us consider the scope of the bound variables in the loose signature of $N S$. The scope rules for bound variables tell us that the name $p$ in $R(p)$ is bound to $p \in P_{1}(p)$, rather than $p \in P_{0}$, since $p \in P_{1}(p)$ is the closest declaration of $p$. The name $p$ in $S(p, q)$ is bound to $p \in P_{2}(p, q)$ since $p \in P_{2}(p, q)$ is closer than $p \in P_{0}$. The name $p$ in $P_{1}(p)$ and $P_{2}(p, q)$ is bound to $p \in P_{0}$. So, the scope of each bound variable in the loose signature of $N S$ is exactly the desired scope of the corresponding component name in $N S$.

\subsection{Specifications in MLT}

This section considers the translation of specifications. In Section 4.3, we outlined the translation of a specification as being a term in MLT that denotes a dependent product type whose left and right fields are the translation of the signature and restriction, respectively, of the specification. Figure 4.5 also gave an example, $\llbracket M S \rrbracket$, of the translation of the specification, $M S$, for monoids. We now generalise the outline given in Section 4.3, by considering the following specification:

$S P=$ Elements $S$ Restrictions $R\left(y_{1}, \ldots, y_{n}\right)$ End,

We assume that signature $S$ includes components named $y_{1}, \ldots, y_{n}$. The translation of $S P$ is given below with an explanation following:

$$
\llbracket S P \rrbracket=\sum x \in \llbracket S \rrbracket \cdot R\left(x . y_{1}, \ldots, x . y_{n}\right)
$$

$\llbracket S \rrbracket$ denotes the translation of signature $S$, and $R\left(x . y_{1}, \ldots, x . y_{n}\right)$ is the translation of the restriction $R\left(y_{1}, \ldots, y_{n}\right)$. The translation of a restriction is a term in MLT that denotes a proposition-and, hence, a type-dependent on $x \in \llbracket S \rrbracket$. Although a restriction is already a term in MLT that denotes a proposition, its translation is obtained by substituting each free occurrence of component names $y_{i}(1 \leq i \leq n)$ by $x . y_{i}$; recall that the substitution is necessary so that the translation of the restriction can refer to the components $y_{1}, \ldots, y_{n}$ in $x \in \llbracket S \rrbracket$.

\subsubsection{Some Specification Notation}

In the following, we give the formal definition of the judgement spec which is used to determine whether a type is a well-typed specification. Just like the judgement sig, 
the judgement spec is not intended to be a new judgement for the type theory, but rather, it is a meta-judgement that holds whenever a term in MLT is the translation of some specification.

\section{Definition 4.5 (Judgement spec)}

$\sum m \in S . R(m)$ spec iff $S$ sig and $\{m \in S \triangleright R(m)$ type $\rfloor$

Notation If $\sum m \in S . R(m)$ spec then we often write $\langle S \mid(m) R\rangle$ as a shorthand for $\sum m \in S . R(m)$.

The functions $S i g$ and $A x$, defined below, are needed when defining operations and properties over specifications. The function $S i g$ is used to return the signature (in MLT) of a specification. The function $A x$ returns the restriction (in MLT) of a specification. Note that $A x$ actually returns a function: the translations of restrictions are dependent on computational elements satisfying the signature of a specification, so they are functions that take computational elements and return propositions.

\section{Definition 4.6 ( $\mathrm{Sig}$ and $A x$ )}

Given $\sum m \in S . R(m)$ spec then functions $S i g \in U_{1} \rightarrow U_{1}$ and $A x \in \prod X \in U_{1} .(X \rightarrow$ $\left.U_{1}\right)$ are defined as follows:

$$
\begin{array}{llll}
\operatorname{Sig}\left(\sum m \in S . R(m)\right) & =S & & \in U_{1} \\
A x\left(\sum m \in S . R(m)\right) & =\lambda m \in S . R(m) & \in S \rightarrow U_{1}
\end{array}
$$

\section{Fact 4.4 If $S P$ spec then $\operatorname{Sig}(S P)$ sig.}

Remark Functions Sig and Ax are actually defined using the urec operator, and so are total functions over all types, not just specifications. For any non-specification $P$, we define $\operatorname{Sig}(P)=P$ and $A x(P)=\lambda m \in \operatorname{Sig}(P) . T$. However, since we never need to consider the application of $S i g$ and $A x$ to types other than module specifications, we have omitted the full definition of $A x$ and $S i g$ from Definition 4.6.

\subsection{Computational Elements in MLT}

In this section we consider the translation of computational elements. The translations of computational elements are members of the translation of signatures, so they are terms in MLT that denote pairs. A computational element $m$ is said to "meet" (or "satisfy") a signature $S$ if $\llbracket m \rrbracket \in \llbracket S \rrbracket$. The left component of a computational element $\llbracket m \rrbracket \in \llbracket S \rrbracket$ is always the domain of $\llbracket S \rrbracket$, and so-further overloading the word domain - we call it the domain of $\llbracket m \rrbracket$. The right component of $\llbracket m \rrbracket$ is called the value tuple of $\llbracket m \rrbracket$, and is a member of the loose signature of $\llbracket S \rrbracket$. 


\subsubsection{An Example Translation}

We illustrate the translation of computational elements by considering the following:

$$
m=\text { module } G=\operatorname{List}(\mathbb{N}), \oplus=+, i d=\text { nil end } \in S
$$

The computational element $m$ meets the signature, $S$, for monoids, given in Section 4.4. The translation of $m$ is:

$$
\llbracket m \rrbracket=\left\langle\left[\operatorname{inl}\left({ }^{\prime} G \text { ') }, \operatorname{inl}\left({ }^{\prime} \oplus '\right), \operatorname{inl}\left({ }^{\prime} i d^{\prime}\right)\right],\langle\operatorname{List}(\mathbb{N}),\langle+,\langle n i l, t t\rangle\rangle\rangle\right\rangle \in \llbracket S \rrbracket\right.
$$

The left component of $\llbracket m \rrbracket$ is the domain of $\llbracket m \rrbracket$, and is equal to the domain of $\llbracket S \rrbracket$ :

$$
\left[\operatorname{inl}\left({ }^{\prime} G \text { ' }\right), \operatorname{inl}\left({ }^{\prime} \oplus \text { ') } \operatorname{inl}\left({ }^{\prime} i d^{\prime}\right)\right]=\operatorname{Dom}(\llbracket S \rrbracket)\right.
$$

The right component of $\llbracket m \rrbracket$ is the value tuple of $\llbracket m \rrbracket$ and is a member of the loose signature of $\llbracket S \rrbracket$ :

$$
\langle\operatorname{List}(\mathbb{N}),\langle \#,\langle n i l, t t\rangle\rangle\rangle \in \operatorname{Lsg}(\llbracket S \rrbracket)
$$

Note that the final component in a value tuple is always the unit value, $t t$, since loose signatures are always terminated by the unit type $T$. The translation of the empty module, module end, is $\langle$ nil, tt $\rangle$ where nil is the empty domain, and $t t \in T$ is an empty value tuple.

The names in the domain of a computational element are the names of corresponding components in its value tuple. For example, the component $\operatorname{List}(\mathbb{N})$ has the name ' $G$ '; + has name ' $\oplus$ '; and nil has the name ' $i d$ '. The domain of a computational element will be used to define a dot notation so that we can refer to the components in the value tuple by name; for example, $\llbracket m \rrbracket . ' G$ ' refers to $\operatorname{List}(\mathbb{N})$. In this thesis, we will not require to make use of a formal definition of dot notation but, for completeness, one is given in Appendix A.

\subsubsection{Notations for Computational Elements in MLT}

This section give some formal notations on computational elements in MLT.

\section{Definition 4.7 (Signature Satisfaction)}

Let $m$ be a computational element, and $S$ sig. $m$ meets $S$ iff $m \in S$.

Figure 4.6 gives two rules to judge whether a computational element meets a signature. The first rule is for the empty signature, and the second rule is for non-empty signatures. The rules are not new inference rules for the type theory, but are deduced as a consequence of the definition of signatures and computational elements. The rules are justified using the introduction and elimination rules for singleton types and $\sum$-types. Informally, Comp1 and CompQ can be seen as introduction rules for signature in MLT. 


$$
\text { Comp1 }\langle n i l, t t\rangle \in\{n i l\}_{\mathbb{D}} \times T
$$

$$
\text { Comp2 } \frac{a \in \mathbb{S}+\mathbb{S} \quad e \in A \quad\{l\}_{\mathbb{D}} \times B(e) \operatorname{sig} \quad\langle l, b\rangle \in\{l\}_{\mathbb{D}} \times B(e)}{\langle a: l,\langle e, b\rangle\rangle \in\{a: l\}_{\mathbb{D}} \times\left(\sum x \in A . B(x)\right)}
$$

Figure 4.6: Inference rules for Computational elements

We define two projection functions, dom and val, which each take a computational element, and return its domain and value tuple, respectively.

Definition 4.8 ( $d o m$ and $v a l$ )

Given $S$ sig and $m \in S$ then functions $d o m \in S \rightarrow\{\operatorname{Dom}(S)\}_{\mathbb{D}}$ and val $\in S \rightarrow$ $\operatorname{Lsg}(S)$ are defined as follows:

$$
\begin{aligned}
& \operatorname{dom}(m)=f s t(m) \in\{\operatorname{Dom}(S)\}_{\mathbb{D}} \\
& \operatorname{val}(m)=\operatorname{snd}(m) \in \operatorname{Lsg}(S)
\end{aligned}
$$

Fact 4.5 Given $S$ sig and $m \in S$ then $m=\langle\operatorname{dom}(m), \operatorname{val}(m)\rangle \in S \square$

\subsubsection{Dependencies within Computational Elements}

Value tuples do not encode dependencies between components in a computational element, as value tuples are ordinary pair values and cannot express dependencies between their components. We obtain the value tuple of a computational element with dependencies by removing the dependencies using substitution. For example, consider the following computational element, $m_{1}$, in which the component $y_{2}$ is dependent on the component $y_{1}$ :

$$
m_{1}=\text { module } y_{1}=e_{1}, y_{2}=e_{2}\left(y_{1}\right) \text { end, }
$$

The value tuple of $m_{1}$ is $\left\langle e_{1},\left\langle e_{2}\left(y_{1} \backslash e_{1}\right), t t\right\rangle\right\rangle$; here the dependency that $e_{2}$ has on $y_{1}$ is removed by substituting $e_{1}$, the value bound to $y_{1}$, for occurrences of $y_{2}$ in $e_{2}$. The rules governing the order in which dependencies are substituted enforce the scope of each component name within a computational component, and are discussed in section 5.6.1.

\subsection{Modules in MLT}

In this section, we consider modules in MLT. In Section 4.3, we outlined modules in MLT as terms that denote pairs whose left and right components are the translation 
of the computational element and witness, respectively. Section 4.3 also gave an example translation of the module $n m$ satisfying specification $M S$ of monoids. Let us generalise the outline given in Section 4.3. Modules in MLT are members of specifications in MLT, so they are terms in MLT that denote pairs. Consider the following module:

$$
m=\text { module } y_{1}=e_{1}, \ldots, y_{n}=e_{n} \text { proof } p \text { end } \in S P
$$

We assume that $m$ satisfies the specification $S P$ :

$$
S P=\text { Elements } S \text { Restrictions } R\left(y_{1}, \ldots, y_{n}\right),
$$

The translation of $m$ is given below with an explanation following.

$$
\llbracket m \rrbracket=\left\langle\llbracket \text { module } y_{1}=e_{1}, \ldots, y_{n}=e_{n} \text { end } \rrbracket, p\right\rangle \in \llbracket S P \rrbracket
$$

where

$$
\begin{array}{lll}
f s t(\llbracket m \rrbracket) & \in & S i g(\llbracket S P \rrbracket) \\
s n d(\llbracket m \rrbracket) & \in & A x(\llbracket S P \rrbracket)(f s t(\llbracket m \rrbracket)
\end{array}
$$

The left component of $\llbracket m \rrbracket$ is the translation of the computational element containing the components in $m$, and the right component is the translation of the witness of $m$. The witness of a module is already a term in MLT, and is syntactically identical to its translation.

\subsubsection{Some Module Notation}

We now define some notation for modules in MLT.

\section{Definition 4.9 (Specification Satisfaction)}

Let $m$ be a module, $S P$ spec. $m$ is said to satisfy (or implement) $S P$ iff $m \in S P$.

We define two projection functions, $c e$ and $p f$, which return the computational element and witness, respectively, of a module. The definitions of $c e$ and $p f$ are given by Definition 4.10. Note the use of the П-type constructor to give the type of $p f: p f$ is a dependent function since the type of a witness is dependent on the computational element of a module. Definition 4.10 is worth remembering as it is often used in proofs concerning specifications.

Definition 4.10 ( $c e$ and $p f$ )

Given $S P$ spec and $m \in S P$ then functions $c e$ and $p f$ are defined as follows:

where

$$
\begin{aligned}
& c e(m)=f s t(m) \\
& p f(m)=\operatorname{snd}(m)
\end{aligned}
$$

$$
\begin{aligned}
& c e \in S P \rightarrow \operatorname{Sig}(S P) \\
& p f \in \prod m \in S P .(A x(S P)(c e(m)))
\end{aligned}
$$

Fact 4.6 Given $S P$ spec and $m \in S P$ then $m=\langle c e(m), p f(m)\rangle \in S P \square$ 


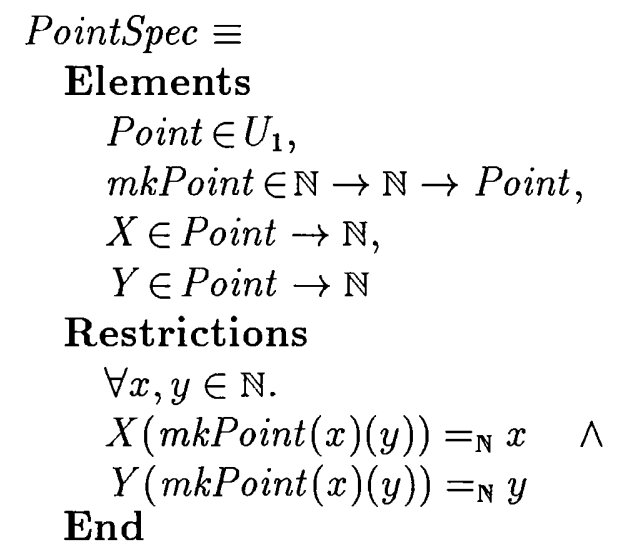

Figure 4.7: The Specification of Cartesian Points



Figure 4.8: An Implementation for PointSpec

\subsection{An Example Translation: PointSpec}

In this section, we exemplify the use of the notations defined in this chapter by giving an example specification and module, and their corresponding translations in MLT. The specification PointSpec, given in Figure 4.7, specifies a module that contains a type to represent points on a plane. PointSpec also specifies an operation to make a Point value from a Cartesian representation, as well as operations to return the $x$ - and $y$-coordinates for Cartesian equivalents of a Point value. The module PointModule, defined in Figure 4.8, gives one possible implementation for PointSpec (i.e $\llbracket$ PointModule $\in \llbracket$ PointSpec $)$ ). PointModule is made by choosing to represent Point values as pairs of natural numbers.

Let us consider the translations of PointSpec and PointModule. The translation of

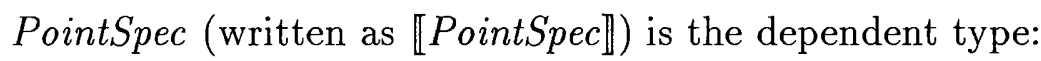

$$
\sum m \in \operatorname{Sig}(\llbracket \text { PointSpec } \rrbracket) . A x(\llbracket \text { PointSpec } \rrbracket)(m)
$$

Here, the translations of the signature and restriction of PointSpec are elaborated by 
the following equations:

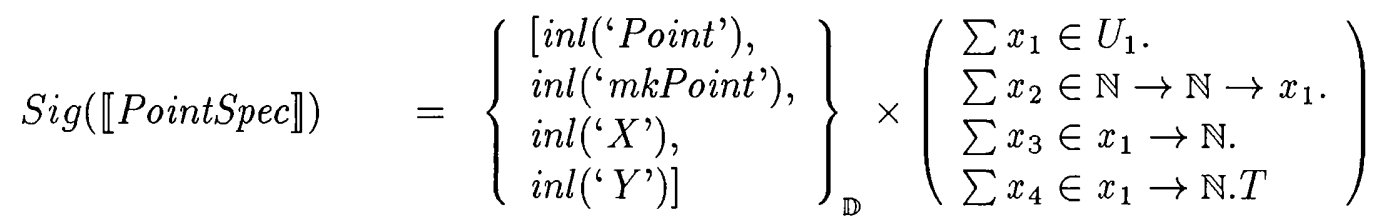

$$
\begin{aligned}
& \forall x, y \in \mathbb{N} . \\
& A x(\llbracket \text { PointSpec } \rrbracket)(m)=m \cdot X(m \cdot m k P o i n t(x)(y))=_{\mathbb{N}} x \wedge \\
& m \cdot Y(m . m k P o i n t(x)(y))={ }_{\mathrm{N}} y
\end{aligned}
$$

The translation of PointModule (written as $\llbracket$ PointModule $\rrbracket$ ) and is the pair:

$$
\langle c e(\llbracket \text { PointModule } \rrbracket), p f(\llbracket \text { PointModule } \rrbracket)\rangle \in \llbracket \text { PointSpec } \rrbracket
$$

here

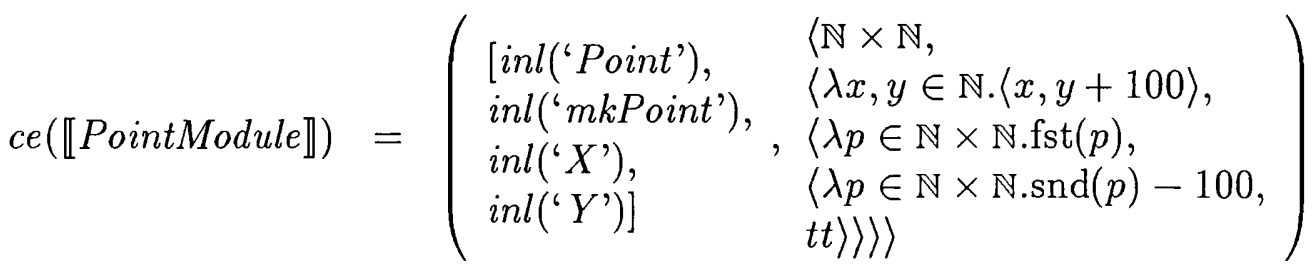

$$
\begin{aligned}
& p f(\llbracket \text { PointModule } \rrbracket)=\lambda x \in \mathbb{N} \cdot \lambda y \in \mathbb{N} .\langle e q, e q\rangle
\end{aligned}
$$

\subsection{Type and Specification Equality}

One of the problems with using types as propositions is that intuitionistic logic does not always obey the properties of classical logic; for example, intuitionistic logic does not obey the law of the excluded middle (i.e. $P \vee \neg P$ is not always true). More importantly for us, properties such as $\wedge$-associativity and $\wedge$-commutativity do not hold with respect to type equality. Consequently, a specification of the form $\langle S \mid(m) P \wedge Q\rangle$ may not be equal to $\langle S \mid(m) Q \wedge P\rangle$ since $P \wedge Q=Q \wedge P$ may not be true; note that here we are using the shorthand notation for specifications, which we defined immediately after Definition 4.5. However, we would like the specifications $\langle S \mid(m) P \wedge Q\rangle$ and $\langle S \mid(m) Q \wedge P\rangle$ to be defined as equivalent since they admit the same implementations (disregarding witnesses). In this section, we define a specification equality, based on the notion of weak type equality, which admits many equivalences-such as commutativity—not allowed by type equality.

\subsubsection{Weak Type Equality}

In Martin-Löf's type theory-and other type theories - the type equality given by the theory is not always suitable for comparing propositions. It is more usual to use 
weak type equality as an equality test between propositions. Informally, two types $P$ and $Q$ are said to be weakly equivalent, written $P \Leftrightarrow Q$, if we can always generate a member of $Q$ from a member of $P$, and vice versa. We use the following definition of weak type equality:

\section{Definition 4.11 (Weak Type Equality $\Leftrightarrow$ )}

Given two types $P$ and $Q$, then $P \Leftrightarrow Q$ iff there exists some functions $f \in P \rightarrow Q$ and $g \in Q \rightarrow P$.

Weak type equality is an equivalence: it is reflexive, symmetric and transitive. We do not give all the properties of weak type equality as they are reasonably well described in the literature; it admits many of the equalities of classical logic, but not the law of the excluded middle.

Fact 4.7 $P=Q$ implies $P \Leftrightarrow Q \square$

Fact $4.8 P \wedge Q \Leftrightarrow Q \wedge P \square$

Fact $4.9 P \wedge(Q \wedge R) \Leftrightarrow(P \wedge Q) \wedge R \square$

Fact $4.10 P \wedge P \Leftrightarrow P \square$

In the following, when we say two restrictions, or propositions, are equivalent, we mean equivalent with respect to weak type equality; for example, $P$ is equivalent to $Q$ means $P \Leftrightarrow Q$.

\subsubsection{Specification Equality}

Weak type equality to used to define a practical definition of equality for specifications. We say that two specifications $S P_{1}$ and $S P_{2}$ are equivalent, written $S P_{1} \Leftrightarrow S P_{2}$, whenever the signatures of $S P_{1}$ and $S P_{2}$ are equal and their restrictions are equivalent. Note that we overload the symbol $\Leftrightarrow$ by using it to denote specification equality and weak type equality. Whenever the arguments of $\Leftrightarrow$ are specifications, it denotes specification equality; and if any of its arguments are not specifications then it denotes weak type equality.

\section{Definition 4.12 (Specification Equality)}

Given $S P_{1}$ spec and $S P_{2}$ spec, then $S P_{1} \Leftrightarrow S P_{2}$ iff:

$$
\begin{aligned}
& \operatorname{Sig}\left(S P_{1}\right)=\operatorname{Sig}\left(S P_{2}\right) \text { type } ; \text { and } \\
& \forall m \in \operatorname{Sig}\left(S P_{1}\right) . A x\left(S P_{1}\right)(m) \Leftrightarrow A x\left(S P_{2}\right)(m)
\end{aligned}
$$

Specification equality is an equivalence (Facts 4.11-4.13) and gives us equalities that do not hold under type equality, such as Facts 4.14, 4.15 and 4.16 (justified by Facts $4.8,4.9$, and 4.10 , respectively). 
Fact $4.11 S P \Leftrightarrow S P \square$

Fact 4.12 $S P_{1} \Leftrightarrow S P_{2}$ implies $S P_{2} \Leftrightarrow S P_{1}$

Fact 4.13 $S P_{1} \Leftrightarrow S P_{2}$ and $S P_{2} \Leftrightarrow S P_{3}$ implies $S P_{1} \Leftrightarrow S P_{3} \square$

Fact $4.14\langle S \mid P \wedge Q\rangle \Leftrightarrow\langle S \mid Q \wedge P\rangle \square$

Fact $4.15\langle S \mid P \wedge(Q \wedge R)\rangle \Leftrightarrow\langle S \mid(P \wedge Q) \wedge R\rangle \square$

Fact $4.16\langle S \mid P \wedge P\rangle \Leftrightarrow\langle S \mid P\rangle \square$

In the remainder of this thesis, when we say two specifications are equivalent, we mean equivalent with respect to specification equality ( $\Leftrightarrow$ ); for example, $S P_{1}$ is equivalent to $S P_{2}$ means $S P_{1} \Leftrightarrow S P_{2}$.

\subsection{Some Alternative Semantics}

The types and notations presented in this chapter are not the only way of defining the semantics of specifications and modules in type theory. Our work on the semantics of specifications has led us to contemplate several alternative definitions for specifications in MLT. In this section, we consider some of these alternatives.

\subsubsection{An Alternative Naming Strategy}

We could have chosen alternative ways of defining the semantics of component names within signatures. One option considered was to pair each type in a loose signature with a singleton type containing the name of a component. For example, the translation of a signature of a monoid specification would then be:

$$
\begin{aligned}
& \sum x \in\left(\left\{i n l\left(' G^{\prime}\right)\right\}_{\mathbb{D}} \times U_{1}\right) . \\
& \sum y \in\left(\left\{\operatorname{inl}\left({ }^{\prime} \oplus '\right)\right\}_{\mathbb{D}} \times x \rightarrow x \rightarrow x\right) . \\
& \sum z \in\left(\left\{i n l\left(' i d^{\prime}\right)\right\}_{\mathbb{D}} \times x\right) . T
\end{aligned}
$$

The values in such types are tuples in which each component is paired with a component name. For example, the following is a value of the above type:

$$
\left\langle\left\langle\operatorname{inl}\left({ }^{\prime} G '\right), \operatorname{List}(\mathbb{N})\right\rangle,\left\langle\operatorname{inl}\left({ }^{\prime} \oplus '\right), H\right\rangle,\left\langle\operatorname{inl}\left({ }^{\prime} i d^{\prime}\right), n i l\right\rangle, t t\right\rangle
$$

The alternative semantics, above, are isomorphic to the original semantics of signatures and computational elements. However, the alternative semantics are harder to manipulate since component values are interleaved with their names. In particular, the alternative semantics require the use of projection functions to define operations on the name-space of modules. In contrast, using the original semantics we can define operations on the name-space using list operations. As we shall see later, reasoning 
about projection functions requires extra work to ensure type correctness. However, the advantages of using the original semantics are limited to operations on the namespace, as both the original and alternative semantics require the use of projection functions to define operations over the component values in a module.

\subsubsection{An Alternative Semantics for Specifications}

It is possible to define translations for specifications so that modules satisfying specifications do not contain witnesses; in other words, so that modules are just computational elements. Such translations can be defined using the subset type constructor. For example, given a specification $S P_{1}=\sum m \in S . R(m)$, we can define the type containing just the computational elements satisfying $S P_{1}$ as $S P_{2}=\{m \in S \mid R(m)\}$. The member of the subset type $S P_{2}$ are all the computational elements $m \in S$ such that the proposition $R(m)$ is true.

The problem with using the subset type is that the subset elimination rule does not allow us to assume that a member of $S P_{2}$ satisfies $R(m)$. For example, the only property we can deduce about the members of $S P_{2}$ is that they are members of $S$. The problem generalises to not being allowed to assume that $Q(x)$ is true under the assumption that $x \in\{y \in P \mid Q(y)\}$. The problem is well-known in type theory and stems from the fact that the members of a subset type do not contain witnesses that prove that they satisfy the restrictions of the subset type. Several solutions have been proposed, though none are totally satisfactory for our purposes. We do not discuss the issue further and refer the interested reader to $[46,44]$.

\subsection{Conclusion and Summary}

Although Nordström et al $[43,44]$ have shown that modules can be specified in MLT, they do not give a formal treatment to component names within specifications and modules. The semantics of our specifications and modules include a definition of domains in MLT, so component names have a formal semantics. These semantics are certainly complicated - in comparison to the NPS and deliverables approachesby the inclusion of component names. But this disadvantage is out-weighed by the importance of component names to the development of a usable specification and implementation language. After all, names provide a means by which one specification, or module, can use the operations of another. As we will see in Chapter 6, component names also play an important role in the design of useful specification and module operators. 
One consequence of our semantics for specifications is that if a specification contains local components, then all modules satisfying it contain implementations of the local components. We justify the implementation of local components on the grounds that they are needed to calculate the types of other components in a module; these types are needed to prove a module satisfies its specification. We illustrate this point by considering the specification $S P$, and the module $m \in S P$, both given in Figure 4.9; for simplicity, we assume there are no dependencies between the components in $m$. Note that the type of $y_{3}$, in $S P$, is dependent on the local component $y_{2}$. Therefore, we need $e_{2}$, the implementation of $y_{2}$ in $m$, to calculate the type of $e_{3}\left(e_{3} \in A_{3}\left(e_{2}\right)\right)$; we need to prove $e_{3} \in A_{3}\left(e_{2}\right)$ to prove that $m \in S P$. The restriction of $S P$ is also dependent on $y_{2}$, so we also need $e_{2}$ to prove $p$ is a witness for $m$, i.e. to prove $p \in R\left(e_{1}, e_{2}, e_{3}\right)$. We need to prove $p \in R\left(e_{1}, e_{2}, e_{3}\right)$ to prove $m \in S P$.

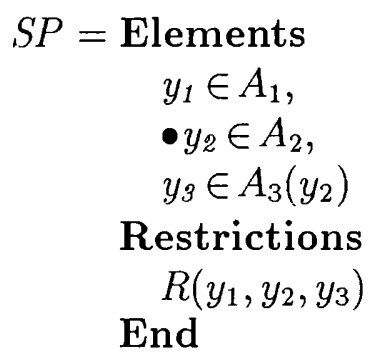

$$
\begin{gathered}
m=\text { module } \\
y_{1}=e_{1}, \\
\bullet y_{2}=e_{2}, \\
y_{3}=e_{3} \\
\text { proof } \\
p \\
\text { end } \in S P
\end{gathered}
$$

Figure 4.9: A Specification $S P$ and its implementation $m \in S P$

There are some disadvantages to defining specifications and modules in a constructive type theory. Firstly, modules contain witnesses that have no use as programs: witnesses are only needed to give constructive proofs about the properties of modules. Secondly, as noted in section 4.9, constructive logics do not obey all the properties of classical logics. This latter point occasionally makes it harder to reason about specifications presented in type theory, compared to those presented in specification languages, such as $\mathrm{Z}$ and CLEAR, which use classical logic.

There are compensating advantages to defining specifications and modules in type theory. Firstly, type theory provides a single framework for constructing specifications and modules. Secondly, the formal language of type theory can be used as a programming logic for reasoning about specifications and modules. Thirdly, the membership relation $(\epsilon)$ provides a simple definition of implementation: a module $m$ satisfies a specification $S P$ if $m \in S P$.

In summary, we have proposed a definition of specifications and modules in MLT, and defined some basic operations to manipulate them. A specification is a type, and the elements of this type are modules satisfying the specification. 


\section{Chapter 5}

\section{The Translation Mapping}

\subsection{Introduction}

Although we discussed the translation of specifications and modules in Chapter 4, and defined the terms used as their translations, we did not define a mapping from specifications and modules to their translations in MLT. We need such a mapping so that we have a formal method of translating specifications and modules correctly when defining and proving laws about them. In this chapter, we define a formal mapping, called the translation mapping, from specifications and modules to their translations in MLT.

Most of the technical details concerning the definition of the translation mapping are easily inferred from the definitions of specifications and modules in MLT, given in Chapter 4. The definition of the translation mapping is slightly complicated over signatures and computational elements, as it must factor signatures into their domains and loose signatures; and computational elements into their domains and value tuples.

We will proceed by giving grammars for the syntax of specifications, modules, signatures and computational elements. Then we define the translation mapping by structural induction on the terms of each grammar. Not all terms defined by the grammars are well-typed, as grammars are only syntax rules, so we will give some laws that use the translation mapping to determine if a term is well-typed.

\subsection{The Syntax of Specifications and Modules}

Figure 5.1 gives the formal syntax for specifications and modules, with an explanation following. We let the tokens $S, S l, b$ and $e$ stand for terms satisfying the grammar sig, siglist, bind and Exp respectively; $R$ and $A$ are tokens for terms satisfying Type; 
Specifications

spec ::= Elements $S$ Restrictions $R$

Signatures

sig $\quad::=\Phi \mid S l$

siglist $::=y \in A|\bullet y \in A| y \in A, S l \mid \bullet y \in A, S l$

Modules

$\bmod ::=\operatorname{module}$ proof $e$ end $\mid \operatorname{module} b$ proof $e$ end

Computational Elements

comp $::=$ module end $\mid \operatorname{module} \mathrm{b}$ end

bind $::=y=e|\bullet y=e| y=e, b \mid \bullet y=e, b$

Types

$\begin{aligned} \text { Type }::= & \mathbb{N}|\mathbb{B}| \mathbb{S}\left|\sum x \in P . Q(x)\right| \Pi x \in P . Q(x)|P+Q| \operatorname{List}(P) \mid \ldots \\ & \mid \text { spec } \mid \text { sig }\end{aligned}$

Expressions

$\operatorname{Exp}::=y \mid$ ' $y$ ' $\mid$ גe.e $\left|e_{1}\left(e_{2}\right)\right| e_{1} . e_{2}\left|\left\langle e_{1}, e_{2}\right\rangle\right| f s t(e)|\operatorname{snd}(e)| \ldots$

$\mid$ Type $|\bmod | \operatorname{comp}$

Primitive classes

$\operatorname{Exp}_{0}$ - Terms of MLT.

Ident - Identifiers (i.e. Sequences of characters)

Figure 5.1: A Summary of Syntax

$x$ and $y$ are token for terms satisfying Ident. The grammar Type admits terms that denote types in specifications and modules, and includes types used as restrictions in specifications. Note that Type admits specifications and signatures as they are also types; for example, specifications are used as types when defining nested specifications. The grammar Exp admits all terms denoting values that can appear in specifications and modules; these include all terms in MLT, including types, as well as specifications, modules, signatures and computational elements. For brevity, we omit to define most of the terms that Type and Exp inherit from MLT, but the omitted terms can be inferred from the formation, introduction and elimination rules of MLT. Note that we assume the existence of a grammar $\operatorname{Exp}_{0}$ defining only terms in MLT.

Let $F$ and $G$ be any grammars. We write $e: E$ to mean that $e$ is a term satisfying grammar $E$, and write $f: E \rightarrow F$ to mean that $f$ is a mapping from terms satisfying $E$ to terms satisfying $F$. Mappings, such as $f$, are rewrite rules on syntactic objects, and should not be confused with functions in MLT. 


$\begin{array}{lll}\llbracket y \rrbracket & = & y \\ \llbracket y^{\prime} y^{\prime} \rrbracket & =\llbracket y \rrbracket \\ \llbracket \lambda x \in(P) \cdot e \rrbracket & =\lambda \llbracket x \rrbracket \in \llbracket P \rrbracket \cdot \llbracket e \rrbracket \\ \llbracket e_{1}\left(e_{2}\right) \rrbracket & =\llbracket e_{1} \rrbracket\left(\llbracket e_{2} \rrbracket\right) \\ \llbracket e_{1}, e_{2} \rrbracket & =\llbracket e_{1} \rrbracket \cdot \llbracket e_{2} \rrbracket \\ \llbracket\left\langle e_{1}, e_{2}\right\rangle \rrbracket & =\left\langle\llbracket e_{1} \rrbracket, \llbracket e_{2} \rrbracket\right\rangle \\ & \vdots \\ \llbracket \mathbb{N} \rrbracket & =\mathbb{N} \\ \llbracket \prod x \in P . Q(x) \rrbracket & =\prod \llbracket x \rrbracket \in \llbracket P \rrbracket \cdot \llbracket Q(x) \rrbracket \\ \llbracket \sum x \in P \cdot Q(x) \rrbracket & =\sum \llbracket x \rrbracket \in \llbracket P \rrbracket \cdot \llbracket Q(x) \rrbracket \\ \llbracket P+Q \rrbracket & =\llbracket P \rrbracket+\llbracket Q \rrbracket \\ \llbracket \operatorname{List}(P) \rrbracket & =\operatorname{List}(\llbracket P \rrbracket)\end{array}$

Figure 5.2: The Translation of Standard terms of MLT

\subsection{The Translation Mapping}

In the following, we will define a mapping $\llbracket-\rrbracket: E x p \rightarrow E x p_{0}$, called the translation mapping. $\llbracket-\rrbracket$ maps specifications, signatures, modules and computational elementswhich are all terms in Exp - to their translations in MLT. But 【-】 also map the other terms in Exp to MLT as they may use specifications and modules as subterms; for example, Exp admits terms denoting functions that use modules in their body. In other words, $\llbracket-\rrbracket$ maps all $e: \operatorname{Exp}$ to terms in MLT by translating any specifications, signatures, modules and computational elements that appear in $e$.

The definition of $\llbracket-\rrbracket$ is by structural induction on terms satisfying Exp. Figure 5.2 gives the definition of $\llbracket-\rrbracket$ on some of the terms of Exp inherited from MLT; $\llbracket \rrbracket$ is defined in a similar manner on the remaining terms inherited from MLT, and the definitions are omitted for brevity. Note that $\llbracket-\rrbracket$ satisfies $\forall e: \operatorname{Exp}_{0} \cdot \llbracket e \rrbracket=e$, i.e., $\llbracket-\rrbracket$ is

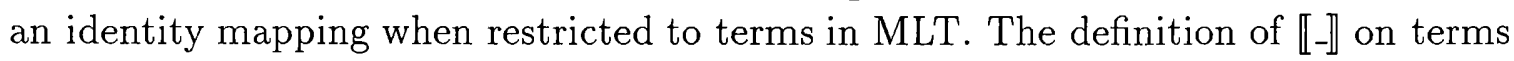
satisfying spec, sig, mod and comp is slightly more complicated, and is the subject of the remainder of this chapter.

\subsection{Translating Signatures}

In this section, we extend the translation mapping, $\llbracket-\rrbracket$, over signatures. The extension is by structural induction on the terms of the grammar sig. Recall that the translation of a signature is the product of its domain and loose signature. Therefore, we will define two mappings, $\llbracket-\rrbracket_{L s g}$ and $\llbracket-\rrbracket_{D o m}$, which translate signatures into their loose signature and domain, respectively; these are used to extend $\llbracket-\rrbracket$ over signatures. The main complication in the definitions of $\llbracket-\rrbracket_{L s g}$ and $\llbracket-\rrbracket_{D o m}$ is ensuring they 
map component identifiers in signatures to bound variables and names, respectively. Hence, we begin by defining the translation of component identifiers.

\subsubsection{Translating Component Identifiers}

In Section 4.4, we noted that the component identifiers in signatures play a dual role as both component names and bound variables. To distinguish between the two roles

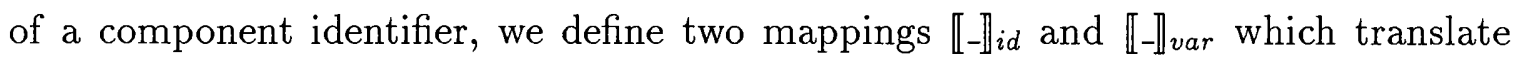
a component identifier- $y$, say-into a component name ' $y$ ' $\in \mathbb{S}$ and a variable $y$, respectively.

Definition $5.1\left(\llbracket-\rrbracket_{i d}\right.$ and $\left.\llbracket-\rrbracket_{v a r}\right)$

Given $y:$ ident, the mappings $\llbracket-\rrbracket_{i d}:$ Ident $\rightarrow$ Exp and $\llbracket-\rrbracket_{v a r}:$ Ident $\rightarrow$ Exp are defined as follows:

$$
\begin{aligned}
& \llbracket y \rrbracket_{i d}=\text { ' } y \text { '; and } \\
& \llbracket y \rrbracket_{\text {var }}=y_{\text {var }}
\end{aligned}
$$

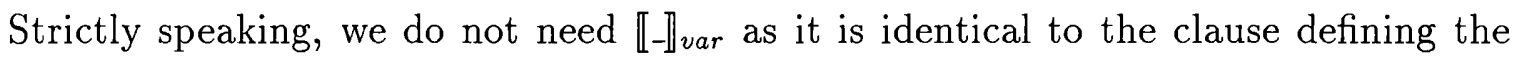
translation mapping over identifiers (i.e. $\llbracket y \rrbracket=y$ ). However, using $\llbracket-\rrbracket$ var emphasizes the translation of an identifier to a variable.

\subsubsection{Translating Loose Signatures}

Definition 5.2 gives a mapping, $\llbracket-\rrbracket_{L s g}$, that translates signatures to their loose signature in MLT. For example, given the signature $S=y_{1} \in A_{1}, \ldots, y_{n} \in A_{n}$, then:

$$
\llbracket S \rrbracket_{L s g}=\sum \llbracket y_{1} \rrbracket_{v a r} \in \llbracket A_{1} \rrbracket \cdot \sum \llbracket y_{2} \rrbracket_{v a r} \in \llbracket A_{2} \rrbracket \ldots \sum \llbracket y_{n} \rrbracket_{v a r} \in \llbracket A_{n} \rrbracket . T
$$

Note that $\llbracket-\rrbracket_{\text {var }}$ translates each component identifiers $y_{i}(1 \leq i \leq n)$ to a bound variable in $\llbracket S \rrbracket_{L s g}$. The translation mapping maps each type $A_{j}(1 \leq j \leq n)$ onto MLT; in the process, each occurrence of $y_{i}$ in $A_{j}$ becomes $\llbracket y_{i} \rrbracket$, and is bound to the corresponding variable declaration $\llbracket y_{i} \rrbracket_{v a r} \in \llbracket A_{i} \rrbracket$.

Definition $5.2\left(\llbracket-\rrbracket_{L s g}\right)$

The mapping $\llbracket-\rrbracket_{L s g}: s i g \rightarrow$ Type is defined inductively, as follows:

$$
\begin{array}{ll}
\llbracket \Phi \rrbracket_{L s g} & =T ; \\
\llbracket y \in A \rrbracket_{L s g} & =\sum \llbracket y \rrbracket_{\text {var }} \in \llbracket A \rrbracket \cdot T ; \\
\llbracket \bullet y \in A \rrbracket_{L s g} & =\sum \llbracket y \rrbracket_{\text {var }} \in \llbracket A \rrbracket . T ; \\
\llbracket y \in A, S \rrbracket_{L s g} & =\sum \llbracket y \rrbracket_{\text {var }} \in \llbracket A \rrbracket . \llbracket S \rrbracket_{L s g} ; \text { and } \\
\llbracket \bullet y \in A, S \rrbracket_{L s g} & =\sum \llbracket y \rrbracket_{\text {var }} \in \llbracket A \rrbracket \cdot \llbracket S \rrbracket_{L s g}
\end{array}
$$




\subsubsection{Translating Domains}

We define a mapping, $\llbracket-\rrbracket_{D o m}$, that translates a signature to its domain, as a type, in MLT. For example, given a signature $S=y_{1} \in A_{1}, \ldots, y_{n} \in A_{n}$ then:

$$
\llbracket S \rrbracket_{D o m}=\left\{\left[\operatorname{inl} l\left(\llbracket y_{1} \rrbracket_{i d}\right), \ldots, \operatorname{inl}\left(\llbracket y_{n} \rrbracket_{i d}\right)\right]\right\}_{\mathbb{D}}
$$

Note that $\llbracket-\rrbracket_{i d}$ translates component identifiers into component names with type $\mathbb{S}-$ contrast this with the use of $\llbracket-\rrbracket_{v a r}$ in the definition of $\llbracket-\rrbracket_{L s g}$, in which component identifiers are translated into variables. $\llbracket-\rrbracket_{D o m}$ is defined in terms of an auxiliary mapping, 【-】 dom , which translates a signature to a domain, as a list: $\llbracket-\rrbracket_{D o m}$ takes a signature- $S$, say-and "lifts" $\llbracket S \rrbracket_{d o m}$ to the domain of $S$, as a type.

Definition $5.3\left(\llbracket-\rrbracket_{d o m}\right)$

The mapping 【- $\rrbracket_{d o m}: s i g \rightarrow$ Exp is defined as follows:

$$
\begin{array}{ll}
\llbracket \Phi \rrbracket_{\text {dom }} & =\text { nil } ; \\
\llbracket y \in A \rrbracket_{\text {dom }} & =\left[\operatorname{inl}\left(\llbracket y \rrbracket_{i d}\right)\right] ; \\
\llbracket \bullet y \in A \rrbracket_{\text {dom }} & =\left[\operatorname{inr}\left(\llbracket y \rrbracket_{i d}\right)\right] ; \\
\llbracket y \in A, S \rrbracket_{\text {dom }} & =\left[\operatorname{inl}\left(\llbracket y \rrbracket_{i d}\right)\right]+\llbracket S \rrbracket_{d o m} ; \\
\llbracket \bullet y \in A, S \rrbracket_{d o m} & =\left[\operatorname{inr}\left(\llbracket y \rrbracket_{i d}\right)\right]+\llbracket S \rrbracket_{\text {dom }} .
\end{array}
$$

\section{Definition $5.4\left(\llbracket-\rrbracket_{D o m}\right)$}

Given any $S:$ sig, the mapping $\llbracket-\rrbracket_{D o m}: s i g \rightarrow$ Type is defined as follows:

$$
\llbracket S \rrbracket_{D o m}=\left\{\llbracket S \rrbracket_{d o m}\right\}_{\mathbb{D}}
$$

\subsubsection{The Translation of Signatures}

We use the mappings $\llbracket-\rrbracket_{L s g}$ and $\llbracket-\rrbracket_{D o m}$ to extend the translation mapping so that it maps signatures to their translations in MLT; recall that the translation of a signature is the product of its domain and loose signature. The extension of the translation mapping over signatures is given by Definition 5.5.

\section{Definition 5.5 (Signature types)}

The translation mapping is extended over all $S:$ sig, as follows:

$$
\llbracket S \rrbracket=\llbracket S \rrbracket_{D o m} \times \llbracket S \rrbracket_{L s g}
$$

We will often write $\llbracket S \rrbracket_{s i g}$ in place of $\llbracket S \rrbracket$ to denote the application of the translation mapping to any $S: \operatorname{sig}\left(\llbracket S \rrbracket_{s i g} \equiv \llbracket S \rrbracket\right)$. 


\subsubsection{Type-Checking Signatures}

The grammar sig gives the syntax rules for signatures, but it does not guarantee that terms satisfying sig are well-typed. For example, the term $x \in \mathbb{N}, y \in\{x\}_{\mathbb{B}}$ is syntactically correct with respect to sig, but is not well-typed: if $x \in \mathbb{N}$ then $\{x\}_{\mathbb{B}}$ is ill-typed since $x$ cannot be a member of both $\mathbb{B}$ and $\mathbb{N}$. If we use a term $S: \operatorname{sig}$ as a type then it must be well-typed; this is checked by using the judgement sig to ensure that $\llbracket S \rrbracket$ sig holds.

In general, determining if $\llbracket S \rrbracket$ sig holds can be broken down into a collection of convenient rules given by the following theorems:

Theorem $5.1 \llbracket \Phi \rrbracket$ sig

Theorem $\mathbf{5 . 2}$ if $\llbracket A \rrbracket$ type then $\llbracket y \in A \rrbracket \operatorname{sig}$

Theorem $\mathbf{5 . 3}$ if $\llbracket A \rrbracket$ type then $\llbracket \bullet y \in A \rrbracket \operatorname{sig}$

Theorem 5.4 if $\llbracket A \rrbracket$ type and $\left\{\llbracket y \rrbracket_{v a r} \in \llbracket A \rrbracket \triangleright \llbracket S \rrbracket\right.$ sig $]$ then $\llbracket y \in A, S \rrbracket$ sig

Theorem 5.5 if $\llbracket A \rrbracket$ type and $\llbracket \llbracket y \rrbracket_{v a r} \in \llbracket A \rrbracket \triangleright \llbracket S \rrbracket$ sig $\rrbracket$ then $\llbracket \bullet y \in A, S \rrbracket$ sig

Proof (of Theorems 5.1-5.5)

Each proof follows from the definition of the mapping 【-』over signatures, and the definition of the judgement sig.

\subsection{Translating Specifications}

In this section, we extend the translation mapping so that it maps specifications into their translations in MLT. Recall, from Section 4.5, that the translation of a specification $S P$,

$$
S P=\text { Elements } y_{1} \in A_{1}, \ldots, y_{n} \in A_{n} \text { Restrictions } R\left(y_{1}, \ldots, y_{n}\right),
$$

is denoted by $\llbracket S P \rrbracket$ :

$$
\llbracket S P \rrbracket=\sum x \in \llbracket y_{1} \in A_{1}, \ldots, y_{n} \in A_{n} \rrbracket_{s i g} \cdot \llbracket R\left(x . y_{1}, \ldots, x . y_{n}\right) \rrbracket
$$

The definition of $\llbracket S P \rrbracket$, given above, virtually defines the translation mapping over specifications. All that is missing is a definition of a mapping that translates restrictions of the form $R\left(y_{1}, \ldots, y_{n}\right)$ to $R\left(m . y_{1}, \ldots, m . y_{n}\right)$. In the following, we define such a mapping, which we call the open mapping, and use it to give the formal definition of the translation mapping over terms satisfying the grammar spec. 


\subsubsection{The Open Mapping}

Definition 5.6 defines the open mapping with explanation following. Given a term satisfying sig, such as $S=y_{1} \in A_{1}, \ldots, y_{n} \in A_{n}$, and a term satisfying Exp, such as $R\left(y_{1}, \ldots, y_{n}\right)$, then:

open $S$ in $R\left(y_{1}, \ldots, y_{n}\right) \equiv[m] R\left(m . y_{1}, \ldots, m . y_{n}\right)$.

Intuitively, open $S$ in $R\left(y_{1}, \ldots, y_{n}\right)$ returns the term obtained by substituting each component identifier $y_{i}(1 \leq i \leq n)$ in $R\left(y_{1}, \ldots, y_{n}\right)$ by $m . y_{i}$. The prefix $[m]$ indicates that $R\left(m . y_{1}, \ldots, m . y_{n}\right)$ is dependent on $m \in S$. The open transformation is analogous to the Pascal with-statement, as it allows the components of a structure $m \in S$ to be used in terms, such as $R\left(y_{1}, \ldots, y_{n}\right)$, without the explicit use of dot notation.

\section{Definition 5.6 (Open)}

The open mapping, open_in_ : sig $\rightarrow$ Exp $\rightarrow$ Exp, is defined recursively:

$$
\begin{array}{ll}
\text { open } \Phi \text { in } e & \equiv[m] e \\
\text { open } y \in A \text { in } e & \equiv[m] e(y \backslash m \cdot[y]) \\
\text { open } \bullet y \in A \text { in } e & \equiv[m] e(y \backslash m \cdot[y]) \\
\text { open } y \in A, S \text { in } e & \equiv \text { open } S \text { in } e(y \backslash m \cdot[y]) \\
\text { open } \bullet y \in A, S \text { in } e & \equiv \text { open } S \text { in } e(y \backslash m \cdot[y])
\end{array}
$$

Unlike other mappings defined in this chapter, open does not always return terms in MLT; in particular, if $e$ is not a term in MLT then neither is open $S$ in $e$. However, we can translate open $S$ in $e$ to MLT by applying the translation mapping ( $\llbracket-\rrbracket)$.

\subsubsection{Translating Specifications}

We use the open mapping to extend the translation mapping so that it maps specifications into their translations in MLT.

\section{Definition 5.7 (Extending 【-』over specifications)}

The translation mapping is extended over terms with syntax spec, as follows:

$\llbracket$ Elements $S$ Restrictions $R \rrbracket=\sum x \in \llbracket S \rrbracket_{s i g}$ 【open $S$ in $R \rrbracket(x)$

Note that we use the translation mapping to translate open $S$ in $R$ into MLT. From the definition of open, we know that open $S$ in $R$ contains a free variable $m \in \llbracket S \rrbracket_{s i g}$.

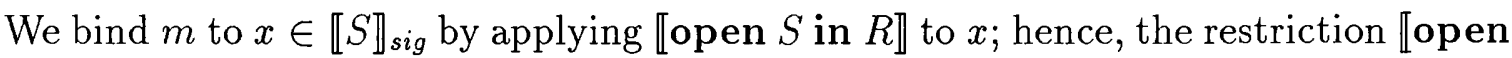
$S$ in $R \rrbracket(x)$ is dependent on $x \in \llbracket S \rrbracket_{\text {sig }}$. 


\subsubsection{Type-Checking Specifications}

The grammar spec only gives the syntax rules for specifications, and does not guarantee type correctness. We check that a term- $S P$, say--satisfying the grammar spec, is well-typed by using the judgement spec defined previously in section 4.5: $S P$ is well typed iff $\llbracket S P \rrbracket$ spec. Determining if $\llbracket S P \rrbracket$ spec holds can be broken down into two requirements. Firstly, the signature of $S P$ must be well-typed. Secondly, the restriction of $S P$ must be a type under the assumption that $S P$ 's signature is well-typed. Theses requirements are stated formally by the following theorem:

Theorem 5.6 (A law about judgement spec)

$\llbracket$ Elements $S$ Restrictions $R \rrbracket$ spec

iff

$\llbracket S \rrbracket_{s i g}$ sig; and

$\llbracket m \in \llbracket S \rrbracket_{\text {sig }} \triangleright \llbracket$ open $S$ in $R \rrbracket(m)$ type $]$.

Proof We omit the proof, but it follows immediately from the translation mapping and the definition of spec.

\subsection{Translating Computational Elements}

In this section, we extend the translation mapping so that it maps computational elements to their translations in MLT. Recall, from Section 4.6, that the translation of a computational element is a pair containing its domain and value tuple. Therefore, we proceed by defining two mappings $\llbracket-\rrbracket_{v a l}$ and $\llbracket-\rrbracket_{d o m}$ which translate computational elements into their value tuple and domain, respectively; these mapping are then used to extend the translation mapping by structural induction on the terms of the grammar comp for computational elements. We will also use the extension to [- \ to give some typing rules for computational elements.

\subsubsection{Translating Value Tuples}

Definition 5.8 gives a mapping, $\llbracket-\rrbracket_{v a l}$, that translates computational elements to their value tuple in MLT. For example, given the computational element:

$$
m=\text { module } y_{1}=e_{1}, \ldots, y_{n}=e_{n} \text { end }
$$

then:

$$
\llbracket m \rrbracket_{v a l}=\left\langle\llbracket e_{1} \rrbracket,\left\langle\llbracket e_{2}\left(y_{1} \backslash e_{1}\right) \rrbracket,\left\langle\ldots,\left\langle\llbracket e_{n}\left(y_{1} \backslash e_{1}, \ldots, y_{n-1} \backslash e_{n-1}\right) \rrbracket, t t\right\rangle \ldots\right\rangle\right\rangle\right\rangle
$$


Note that the translation mapping $(\mathbb{I}-\mathbb{\rrbracket})$ is used to translate each term $e_{i}(1 \leq i \leq n)$ into MLT. The substitutions on each $e_{i}$ remove any dependencies between components in a computational element; this is necessary as the pair constructors used to construct value tuples cannot express dependencies between components. We assume that substitutions of the form $b(y \backslash e)$ only substitute free occurrences of $y$, so that overloaded definitions of $y$ in $b$, and occurrences of $y$ bound to such definitions, are not substituted; this ensures that a component identifier is only substituted by the value it is bound to by the scope rules for computational elements.

Definition $5.8\left(\llbracket-\rrbracket_{\text {val }}\right)$

The mapping $\llbracket-\rrbracket_{v a l}:$ comp $\rightarrow \operatorname{Exp}$ is defined as follows:

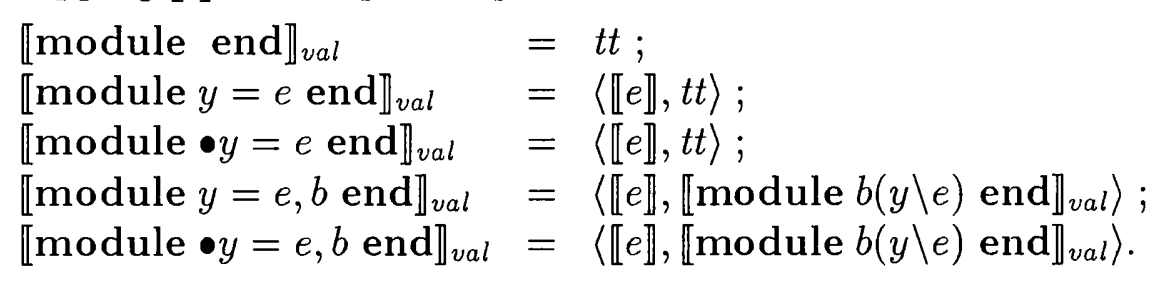

\subsubsection{Translating Domains}

Definition 5.9 gives the mapping $\llbracket-\rrbracket_{d o m}$ that translates computational elements to their domains in MLT. For example, given the computational element $m$-defined in the previous section-then:

$$
\llbracket m \rrbracket_{d o m}=\left[\operatorname{inl} l\left(\llbracket y_{1} \rrbracket_{i d}\right), \ldots, \operatorname{inl} l\left(\llbracket y_{n} \rrbracket_{i d}\right)\right]
$$

Note the use of the mapping $\llbracket-\rrbracket_{i d}$ to translate each component identifier $y_{i}(1 \leq$ in $\leq)$ into a component name of type $\mathbb{S}$.

Definition $5.9\left(\llbracket-\rrbracket_{d o m}\right)$

The mapping $\llbracket-\rrbracket_{d o m}: c o m p \rightarrow E x p$ is defined as follows:



\subsubsection{Translating Computational Elements}

We use the mappings $\llbracket-\rrbracket_{d o m}$ and $\llbracket-\rrbracket_{v a l}$ to extend the translation mapping so that it maps computational elements to their translations in MLT. 


\section{Definition 5.10 (Translating Computational Elements)}

The translation mapping is defined over all $m:$ comp as follows:

$$
\llbracket m \rrbracket=\left\langle\llbracket m \rrbracket_{\text {dom }}, \llbracket m \rrbracket_{\text {val }}\right\rangle
$$

Definition 5.10 is justified by recalling, from Section 4.6, that a computational element in MLT is a pair containing its domain and value tuple.

\subsubsection{Type-Checking Computational Elements}

The grammar comp only gives the syntax rules for computational elements, and does not guarantee type correctness. From the definition of well-typed computational elements (Definition 4.7), it follows that a term $m$ : comp is well-typed with respect to a signature, $\llbracket S \rrbracket$ sig, if $\llbracket m \rrbracket \in \llbracket S \rrbracket$. A judgement of the form $\llbracket m \rrbracket \in \llbracket S \rrbracket$ can be made using theorem 5.7 which recognises the factoring of names and values in the translations of computational elements.

\section{Theorem 5.7}

Let $\llbracket S \rrbracket$ sig hold. $\llbracket m \rrbracket \in \llbracket S \rrbracket$ iff $\llbracket m \rrbracket_{\text {dom }} \in \llbracket S \rrbracket_{\text {Dom }}$ and $\llbracket m \rrbracket_{v a l} \in \llbracket S \rrbracket_{L s g}$.

Proof Theorem 5.7 is justified by the $\times$-introduction rules of the type theory; the proof is trivial, and is omitted.

We also define a collection of laws that allow a judgement $\llbracket m \rrbracket \in \llbracket S \rrbracket$ to be made in a piecewise manner. The laws are given by Theorems 5.8-5.12; informally, the laws can be viewed as introduction rules for signatures.

Theorem $5.8 \llbracket$ module end $\rrbracket \in \llbracket \Phi \rrbracket$

Theorem 5.9 if $\llbracket e \rrbracket \in \llbracket A \rrbracket$ then $\llbracket \operatorname{module} y=e$ end $\rrbracket \in \llbracket y \in A \rrbracket$

Theorem 5.10 if $\llbracket e \rrbracket \in \llbracket A \rrbracket$ then $\llbracket \operatorname{module} \bullet y=e$ end $\rrbracket \in \llbracket \bullet y \in A \rrbracket$

Theorem 5.11 if $\llbracket e \rrbracket \in \llbracket A \rrbracket$ and $\llbracket \operatorname{module} b(y \backslash e)$ end $\rrbracket \in \llbracket S(y \backslash e) \rrbracket$ then $\llbracket$ module $y=e, b$ end $\rrbracket \in \llbracket y \in A, S \rrbracket$

Theorem 5.12 if $\llbracket e \rrbracket \in \llbracket A \rrbracket$ and $\llbracket \operatorname{module} b(y \backslash e)$ end $\rrbracket \in \llbracket S(y \backslash e) \rrbracket$ then $\llbracket$ module $\bullet y=e, b$ end $\rrbracket \in \llbracket \bullet y \in A, S \rrbracket$

Proof (of Theorems 5.8-5.12)

In each case, the proof follows from the definition of the translation mapping and the inference rules Struct1 and Struct2 given previously in Figure 4.6. The proofs are trivial and are omitted. 


\subsection{Translating Modules}

In this section, we extend the translation mapping so that it maps modules to their translations in MLT. Recall, from Section 4.7, that the translation of a module,

$$
m=\text { module } y_{1}=e_{1}, \ldots, y_{n}=e_{n} \text { proof } p \text { end, }
$$

is denoted by $\llbracket m \rrbracket$ :

$$
\llbracket m \rrbracket=\left\langle\llbracket \text { module } y_{1}=e_{1}, \ldots, y_{n}=e_{n} \text { end } \rrbracket, \llbracket p \rrbracket\right\rangle
$$

The definition of $\llbracket m \rrbracket$ is essentially an informal definition of the translation mapping over modules. In the following, we give the formal definition of the translation mapping over modules by structural induction on the terms of the grammar mod for modules.

\subsubsection{Translating Modules}

Definition 5.11 gives the formal definition of the translation mapping over modules.

Definition 5.11 (Extending 【-】 over modules)

The translation mapping is extended over terms satisfying mod, as follows:

$\llbracket \operatorname{module}$ proof $p$ end $\rrbracket=\langle\llbracket \operatorname{module}$ end $\rrbracket, \llbracket p \rrbracket\rangle ;$

$\llbracket$ module $b$ proof $p$ end $\rrbracket=\langle\llbracket$ module $b$ end $\rrbracket, \llbracket p \rrbracket\rangle$

Note that the translation mapping is used to translate both the computational element and witness of a module.

\subsubsection{Type-Checking Modules}

From the definition of well-typed modules (Definition 4.9), it follows that a term $m: \bmod$ is well-typed with respect to a specification $\llbracket S P \rrbracket$ spec if $\llbracket m \rrbracket \in \llbracket S P \rrbracket$. Theorems 5.13 and 5.14 are used to make judgements of the form $\llbracket m \rrbracket \in \llbracket S P \rrbracket$ by factoring them into two separate judgements.

Theorem 5.13

If $\operatorname{Sig}(\llbracket S P \rrbracket)=\Phi$ and $\llbracket p \rrbracket \in A x(\llbracket S P \rrbracket)(\llbracket$ module end $\rrbracket)$ then $\llbracket$ module proof $p$ end $\rrbracket \in \llbracket S P \rrbracket$

\section{Theorem 5.14}

If $\llbracket$ module b end $\rrbracket \in S i g(\llbracket S P \rrbracket)$ and $\llbracket p \rrbracket \in A x(\llbracket S P \rrbracket)(\llbracket$ module b end $\rrbracket)$ then $\llbracket$ module $b$ proof $p$ end $\rrbracket \in \llbracket S P \rrbracket$ 


\section{Proof (of Theorems 5.13 and 5.14)}

The proof follows immediately from Definition 4.9, and is omitted.

\subsection{Conclusion and Summary}

Although the semantics of our specification language is presented in a denotational style, it is not a conventional denotational semantics. In conventional denotational semantics, such as [53], a language is mapped onto the Lambda calculus. However, the translation mapping maps specifications and modules onto Martin-Löf's type theory. Denotational semantics use the Lambda calculus because its properties are well-known and can be used to reason about languages expressed in terms of it. However, the Lambda calculus has its limitations, as many modern programming concepts, such as parallelism and specifications, are difficult to express in the Lambda calculus; for more details see [40].

In summary, we have presented a formal mapping from the syntax of specifications and modules to their translations in Martin-Löf's Type Theory. We have used the mapping to give some laws for type-checking specifications and modules in a piecewise manner. 


\section{Chapter 6}

\section{Signature and Computational Element Operators}

\subsection{Introduction}

Canonical specifications and canonical modules are unsuited to making very large specifications and modules. For example, understanding a canonical specification is difficult if there are too many components in its signature, or too many axioms in its restriction. A more convenient way of making large specifications and modules is to construct them by combining smaller specifications and modules, respectively. Such a style encourages us to make specifications and modules incrementally, as well as helping us to decompose specifications and modules into more manageable pieces. We call specifications constructed from smaller specifications structured specifications; and we call modules constructed from smaller modules structured modules. In Chapter 7 , we will define specification operators that combine and modify specifications to make structured specifications. In order to define such specification operators we will require operators that combine and modify signatures and computational elements. That is the subject of this chapter.

The signature operations we define are signature renaming, signature hiding and signature concatenation. Signature renaming renames components within signatures. Signature hiding hides visible components in a signature by making them local components. Signature concatenation combines two signatures to make a signature containing the components from both signatures. We also define renaming, hiding and concatenation operators on computational elements.

In order to define the signature concatenation operator--which we write as an infix binary operator $\left(\oplus_{-} \oplus_{-}\right)_{-}$we will introduce dependent signatures in MLT. Dependent signatures are signatures whose components depend on the components of other signatures. The signature concatenation operator requires dependent signatures because 
for any signature concatenation, $S_{1} \oplus S_{2}$ for example, we allow $S_{2}$ to be dependent on the components supplied by $S_{1}$. We allow such dependencies since we often use $\oplus$ to enrich signatures with new components defined in terms of components from other signatures.

The resolution of name clashes is another important issue in the design of the signature concatenation operator. Name clashes can arise when we concatenate two signatures that have some component names in common. Name clashes must be resolved as otherwise they can cause ambiguities in signatures. The computational element concatenation operator must also resolve potential name clashes.

In order to avoid the proliferation of operator symbols, we will overload the symbol for each signature operator and also use it for the corresponding computational element operator; for example, we will use $\oplus$ to denote both the signature concatenation and computational element concatenation operators. It will always be clear from the context whether we are using such operator symbols to denote signature operators or computational element operators.

We will also give some laws about each operator defined in this chapter, and these will be used, later, to prove laws about specification and module operators. However, for reasons of space, we do not supply a proof for all the laws given in this chapter.

\subsection{Dependent Signatures}

We begin by defining dependent signatures. A dependent signature is a signature whose components are dependent on those of another signature. For example, $S_{2}$ (below) is dependent on $S_{1}$ as it is defined using $S q r$ in $S_{1}$.

$$
\begin{aligned}
& S_{1}=S q r \in U_{1}, \text { size } \in S q r \rightarrow \mathbb{N} \\
& S_{2}=\text { area } \in S q r \rightarrow \mathbb{N}, \text { size } \in S q r \rightarrow \mathbb{N}
\end{aligned}
$$

Strictly speaking, a dependent signature is not a signature, but an abstraction (i.e. an expression containing free component names) whose free component names can be instantiated to produce a signature.

\subsubsection{The Dependent Signature Judgement}

A dependent signature in MLT — dependent on some signature $S$, for example - is any expression of the form $\{l\}_{\mathbb{D}} \times Q$ where $\{l\}_{\mathbb{D}}$ is a domain, and $Q$ is an abstraction that can be instantiated by the components in the loose-signature of $S$ to produce a loose signature. Given any loose signature

$$
\operatorname{Lsg}(S)=\sum y_{1} \in A_{1} \ldots \sum y_{n} \in A_{n} . T
$$


$Q$ can be instantiated as a loose signature if, and only if, the following holds:

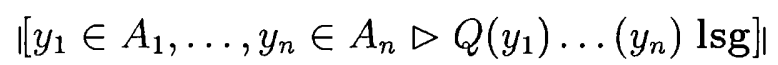

To formally define dependent signatures, we introduce the dependent loose-signature judgement. This has the form $P \vdash Q$ lsg for any $P$ lsg and $Q$ an abstraction. $P \vdash Q$ lsg holds if, and only if, $Q$ is a loose-signature when instantiated by the components supplied by $P$. For example, condition (1) (above) is equivalent to $\operatorname{Lsg}(S) \vdash Q$ lsg.

\section{Definition 6.1 (Dependent Loose-signature)}

Given $\sum x \in A . B(x)$ lsg, then

$$
\begin{array}{ll}
T \vdash Q \text { lsg } & =Q \text { lsg } \\
\left(\sum x \in A . B(x)\right) \vdash Q \text { lsg } & =\llbracket x \in A \triangleright(B(x) \vdash Q(x) \text { lsg }) \rrbracket
\end{array}
$$

We give a formal definition of dependent signatures by defining the dependent signature judgement. This has the form $S_{1} \vdash S_{2}$ sig; it holds if, and only if, $S_{2}$ is a dependent signature that is dependent on $S_{1}$.

\section{Definition 6.2 (Dependent Signatures)}

$S \vdash\{l\}_{\mathbb{D}} \times Q$ sig iff $S$ sig, $l \in \mathbb{D}$ and $(\operatorname{Lsg}(S) \vdash Q$ lsg $)$.

Here are a few useful facts about the dependent signature judgement:

Fact $6.1 \llbracket \Phi \rrbracket \vdash \llbracket S \rrbracket \operatorname{sig}=\llbracket S \rrbracket \operatorname{sig} \square$

Fact $6.2 \llbracket y \in A \rrbracket \vdash \llbracket S \rrbracket \operatorname{sig}=\llbracket \llbracket y \rrbracket \in \llbracket A \rrbracket \triangleright \llbracket S \rrbracket \operatorname{sig} \rrbracket \square$

Fact $6.3 \llbracket \bullet y \in A \rrbracket \vdash \llbracket S \rrbracket \operatorname{sig}=\llbracket \llbracket \llbracket y \rrbracket \in \llbracket A \rrbracket \triangleright \llbracket S \rrbracket \operatorname{sig}] \square$

Fact $6.4 \llbracket y \in A, S_{1} \rrbracket \vdash \llbracket S \rrbracket \operatorname{sig}=\llbracket \llbracket y \rrbracket \in \llbracket A \rrbracket \triangleright\left(\llbracket S_{1} \rrbracket \vdash \llbracket S \rrbracket \operatorname{sig}\right) \rrbracket \mid$

Fact $6.5 \llbracket \bullet y \in A, S_{1} \rrbracket \vdash \llbracket S \rrbracket \operatorname{sig}=\llbracket \llbracket y \rrbracket \in \llbracket A \rrbracket \triangleright\left(\llbracket S_{1} \rrbracket \vdash \llbracket S \rrbracket \operatorname{sig}\right) \rrbracket \mid$

\subsubsection{Signature Instantiation}

Let $S_{2}$ be a dependent signature that is dependent on $S_{1}$. Given any computational element $m \in S_{1}$, we can instantiate each free occurrence of a component name- $p$, for example-in $S_{2}$ by the corresponding component m.p in $m$, so that $S_{2}$ becomes a non-dependent signature. We write such instantiations as $S_{2}(\mid m)_{S_{1}}$ and call them signature instantiations. In this section, we give a formal definition of signature instantiation.

We give some examples of signature instantiation in Facts 6.6-6.10 below.

Fact 6.6 $S(\mid \text { module end })_{\Phi}=S$

Fact 6.7 $S\left(\mid\right.$ module $y=e$ end $D_{y \in A}=S(e)$ 
Fact $6.8 S(\mid \bmod u l e \bullet y=e$ end $) \cdot y \in A=S(e) \square$

Fact $6.9 S\left(\mid\right.$ module $y=e, b$ end $\|_{y \in A, S_{1}}=S(e)\left(\mid\right.$ module $b(y \backslash e)$ end $\|_{S_{1}(y \backslash e)}$

Fact $6.10 S(\mid \operatorname{module} \bullet y=e, b$ end $) \bullet y \in A, S_{1}=S(e)(\mid \operatorname{module} b(y \backslash e) \text { end })_{S_{1}(y \backslash e)}$

We define signature instantiation using loose-signature instantiation. An application of loose-signature instantiation has the form $Q\langle p\rangle_{P}$ where $P$ lsg, and $p \in P$ is a value tuple, and $P \vdash Q$ lsg holds. $Q \backslash p\rangle_{P}$ is $Q$ applied to each component value in $p$, so that $Q$ becomes instantiated as a non-dependent loose-signature. For example, if $p=\left\langle e_{1},\left\langle\ldots,\left\langle e_{n}, t t\right\rangle\right\rangle\right\rangle \in P$ then $Q\langle p\rangle_{P}=Q\left(e_{1}\right) \ldots\left(e_{n}\right)$.

\section{Definition 6.3 (Loose-signature Instantiation)}

Given $P$ lsg, $\sum x \in A . B(x) \vdash Q$ lsg and any $\langle a, b\rangle \in \sum x \in A . B(x)$ then:

$$
\begin{array}{ll}
P\langle\mid t t\rangle_{T} & =P \\
Q\langle\mid\langle a, b\rangle\rangle_{\sum x \in A . B(x)} & =Q(a)\langle b\rangle_{B(a)}
\end{array}
$$

\section{Definition 6.4 (Signature Instantiation)}

Given $S$ sig, $S \vdash\{l\}_{\mathbb{D}} \times Q$ sig and any $m \in S$ then:

$$
\left(\{l\}_{\mathbb{D}} \times Q\right)(\mid m)_{S}=\{l\}_{\mathbb{D}} \times Q \backslash \operatorname{val}(m) D_{L s g(S)} \text { sig }
$$

\subsection{Signature Renaming}

In this section, we define the signature renaming operator. An application of this operator has the form $S[p \backslash q]$ for $p$ and $q$ any component names and $S$ a signature. $S[p \backslash q]$ is $S$ with component $p$ renamed to $q$. An attempt to rename a non-existent component leaves a signature unchanged. Consider the following signature:

$$
S_{1}=\text { Book } \in U_{1}, \text { Stock } \in U_{1}, \text { add } \in \text { Book } \rightarrow \text { Stock } \rightarrow \text { Stock }
$$

Renaming Book to Video, in $S_{1}$, is written as $S_{1}[B o o k \backslash V i d e o] . S_{1}[B o o k \backslash V i d e o]$ can be expressed in canonical form as follows:

$$
S_{1}[\text { Book } \backslash \text { Video }]=\text { Video } \in U_{1} \text {, Stock } \in U_{1} \text {, add } \in \text { Video } \rightarrow \text { Stock } \rightarrow \text { Stock }
$$

An attempt to rename an overloaded component name in any signature, $S$ for example, only renames the first component that has that overloaded name in $S$. For example, given a signature $S=\left(y \in A_{1}, p \in A_{2}, p \in A_{3}\right)$, in which $p$ is overloaded, then $S[p \backslash q]=\left(y \in A_{1}, q \in A_{2}, p \in A_{3}\right)$. Note that $p \in A_{3}$ remains unchanged in $S[p \backslash q]$. This feature of renaming allows each component with an overloaded name to be given a distinct name. For example, $(S[p \backslash q])[p \backslash r]$ renames $p \in A_{2}$ to $q$ and $p \in A_{3}$ to $r$. 


\subsubsection{A Formal Definition of Signature Renaming}

We define signature renaming in Definition 6.5, with explanation following. Signature renaming only modifies the domain of a signature; the loose signature remains unchanged. Signature renaming is defined using the domain substitution function, given in Definition 6.7, an application of which has the form $l[p \| q]$ for any $p, q \in \mathbb{S}$ and $l \in \mathbb{D}$ a domain. $l[p \rrbracket q]$ is $l$ with the first tagged occurrence of $p$ (i.e. the first occurrence of $\operatorname{inl}(p)$ or $\operatorname{inr}(p))$ replaced by $q$, where $q$ is tagged with the same tag that $p$ has. Note that we use the notation given in Definition 6.6 to define the right-hand side of the equality in Definition 6.5; see the remark below. The notation overloads the signature renaming notation in our specification language. To disambiguate this overloading, if $S$ sig (i.e. $S$ is a type) then $S[p \backslash q]$ is a use of the notation in Definition 6.6, but if $S$ is a signature term of our specification language then so is $S[p \backslash q]$.

\section{Definition 6.5 (Signature Renaming)}

Let $S$ be a signature in our specification language (i.e. $\llbracket S \rrbracket \operatorname{sig}$ ) and $\llbracket p \rrbracket, \llbracket q \rrbracket \in \mathbb{S}$.

$$
\llbracket S[p \backslash q] \rrbracket=\llbracket S \rrbracket[\llbracket p \rrbracket \backslash \llbracket q \rrbracket]
$$

\section{Definition 6.6 (Notation for Signature Renaming in MLT)}

Given $S$ sig and $p, q \in \mathbb{S}$ then $S[p \backslash q]=\{\operatorname{Dom}(S)[p \backslash q]\}_{\mathbb{D}} \times \operatorname{Lsg}(S)$

\section{Definition 6.7 (Domain Substitution)}

Let $a, p, q \in \mathbb{S}$ and $l \in \mathbb{D}$. The domain substitution function $(-[-\|-]) \in \mathbb{D} \rightarrow \mathbb{S} \rightarrow \mathbb{S} \rightarrow \mathbb{D}$ is defined as follows:

$$
\begin{aligned}
& \operatorname{nil}[p \| q] \\
& (\operatorname{inl}(a): l)[p \rrbracket q]= \begin{cases}\operatorname{inl}(q): l & \text { if } a=p \\
\operatorname{inl}(a):(l[p \Downarrow q]) & \text { if } a \neq p\end{cases} \\
& (\operatorname{inr}(a): l)[p \| q]= \begin{cases}\operatorname{inr}(q): l & \text { if } a=p \\
\operatorname{inr}(a):(l[p \| q]) & \text { if } a \neq p\end{cases}
\end{aligned}
$$

Remark It is possible to define signature renaming without introducing Definition 6.6. However, its introduction gives us a concise way of denoting expression of the form $\{\operatorname{Dom}(S)[p \rrbracket q]\}_{\mathbb{D}} \times \operatorname{Lsg}(S)$ which will appear frequently in theorems, proofs and definitions. We will introduce similar notations for each of the other signature, and computational element, operations and use them to define the respective signature, or computational element, operation. In each case, the new notation overloads the notation for the corresponding signature, or computational element, operator, but it should always be clear from the context how to disambiguate the overloading. 


\subsubsection{Laws of Signature Renaming}

The application of signature renaming to any canonical signature can be unfolded to regain a canonical signature. This statement is justified by Theorems 6.1-6.5. For example, Theorem 6.4 gives a rule, defined in terms of textual substitution, for applying signature renaming to a non-empty canonical signature; in Theorem 6.4 , the expression $S(p \backslash q)$ stands for $S$ with each free occurrence of $p$ replaced by $q$. Theorems 6.1-6.5 constitute a complete set of rules for calculating the result of applying signature renaming to any canonical signature.

Theorem 6.1 $\Phi[p \backslash q]=\Phi$

Proof Omitted $\square$

Theorem 6.2 $(y \in A)[p \backslash q]= \begin{cases}y \in A & \text { if } p \neq y \\ q \in A & \text { if } p=y\end{cases}$

Proof Similar to that of Theorem 6.4 below.

Theorem 6.3 $(\bullet y \in A)[p \backslash q]= \begin{cases}\bullet y \in A & \text { if } p \neq y \\ \bullet q \in A & \text { if } p=y\end{cases}$

Proof Similar to that of Theorem 6.4 below.

Theorem 6.4 $(y \in A, S)[p \backslash q]= \begin{cases}y \in A,(S[p \backslash q]) & \text { if } p \neq y \\ q \in A,(S(p \backslash q)) & \text { if } p=y\end{cases}$

Proof The proof is given in Figure 6.1. After the second step of the proof, the proof proceeds in two parts which consider the cases $p=y$ and $p \neq y$, respectively $\square$ Theorem 6.5 $(\bullet y \in A, S)[p \backslash q]= \begin{cases}\bullet y \in A,(S[p \backslash q]) & \text { if } p \neq y \\ \bullet q \in A,(S(p \backslash q)) & \text { if } p=y\end{cases}$

Proof Similar to that of Theorem 6.4 above.

\subsection{Signature Hiding}

In this section, we define the signature hiding operator. An application of this operator has the form $S \backslash i$ for $S$ any signature and $i$ a set of component names. $S \backslash i$ is $S$ with each visible component named in $i$ redefined as a local component. For example, consider the following signature:

$$
S_{1}=s q r \in \mathbb{N} \rightarrow \mathbb{N}, \bullet p i \in \mathbb{N}, \text { area } \in \mathbb{N} \rightarrow \mathbb{N}
$$

$S_{1} \backslash\{s q r, p i\}$ is the signature $S_{1}$ with the components $s q r$ and $p i$ made local. Note that $p i$ is already local to $S_{1}$ and it remains local in $S_{1} \backslash\{s q r, p i\}$. The canonical form of $S_{1} \backslash\{s q r, p i\}$ is defined below:

$$
S_{1} \backslash\{s q r, p i\}=\bullet s q r \in \mathbb{N} \rightarrow \mathbb{N}, \bullet p i \in \mathbb{N} \text {, area } \in \mathbb{N} \rightarrow \mathbb{N}
$$

If a component name, $p$ for example, is overloaded in a signature $S$ then $S \backslash\{p\}$ hides all the components named $p$ in $S$. If we wish to keep some of the components named $p$ visible, then these should be temporarily renamed prior to applying signature hiding. 


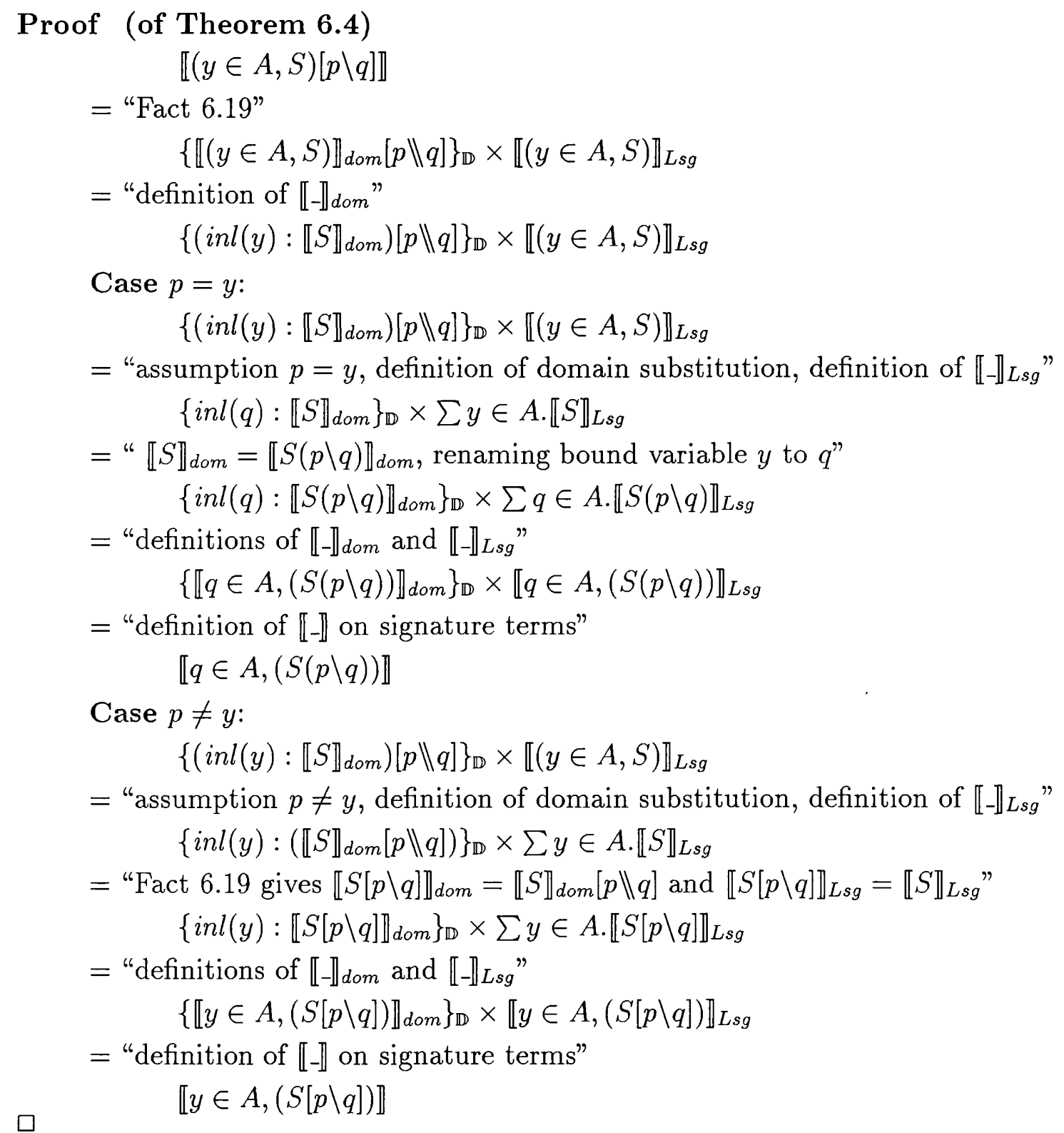

Figure 6.1: A proof of Theorem 6.4 


\subsubsection{A Formal Definition of Signature Hiding}

Like signature renaming, signature hiding only changes the domain of a signature. Signature hiding is defined using the domain hiding function given in Definition 6.10, and the notation given in Definition 6.9 (recall the remark in Section 6.3.1). An


set of component names. For all names $y \in i, l \rrbracket i$ is $l$ with each visible name $\operatorname{inl}(y)$, in $l$, replaced by a local name $\operatorname{inr}(y)$. The formal definition of signature hiding is given in Definition 6.8 below.

\section{Definition 6.8 (Signature Hiding)}

Let $S$ be a signature in our specification language (i.e. $\llbracket S \rrbracket \operatorname{sig}$ ), and $\llbracket i \rrbracket \in \operatorname{Set}(\mathbb{S}$ ).

$$
\llbracket S \backslash i \rrbracket=\llbracket S \rrbracket \backslash \llbracket i \rrbracket
$$

\section{Definition 6.9 (Notation for Signature Hiding in MLT)}

Given $S$ sig and $i \in \operatorname{Set}(\mathbb{S})$ then $S \backslash i=\{\operatorname{Dom}(S) \rrbracket i\}_{\mathbb{D}} \times \operatorname{Lsg}(S)$

\section{Definition 6.10 (Domain Hiding)}

Let $y \in \mathbb{S}, l \in \mathbb{D}$ and $i \in \operatorname{Set}(\mathbb{S})$. The domain hiding function $\left(\mathbb{-}_{-}\right) \in \mathbb{D} \rightarrow \operatorname{Set}(\mathbb{S}) \rightarrow \mathbb{D}$ is defined as follows:

$$
\begin{aligned}
& n i l \rrbracket i \\
& (\operatorname{inl}(y): l) \rrbracket i= \begin{cases}\operatorname{inr}(y):(l \rrbracket i) & \text { if } y \in i \\
\operatorname{inl}(y):(l \rrbracket i) & \text { if } y \notin i\end{cases} \\
& (\operatorname{inr}(y): l) \rrbracket i=\operatorname{inr}(y):(l \rrbracket i)
\end{aligned}
$$

\subsubsection{Laws of Signature Hiding}

If $S$ is a canonical signature, then the result of a signature hiding $S \backslash i(i \in \operatorname{Set}(\mathbb{S}))$ can always be expressed by putting bullets $(\bullet)$ in front of all the visible components of $S$ which are named in $i$. This property is justified by Theorems $6.6-6.10$ below; these theorems are concerned with unfolding applications of signature hiding to produce canonical signatures.

Theorem $6.6 \quad \Phi \backslash i=\Phi$ 


\section{Proof}

$$
\begin{aligned}
& \llbracket \Phi \backslash i \rrbracket \\
& =\text { "definition of signature hiding" } \\
& \{\operatorname{Dom}(\llbracket \Phi \rrbracket) \rrbracket i\}_{\mathbb{D}} \times \operatorname{Lsg}(\llbracket \Phi \rrbracket) \\
& =" \operatorname{Dom}(\llbracket \Phi \rrbracket)=n i l \text { and } n i l \rrbracket i=n i l, L s g(\llbracket \Phi \rrbracket)=T " \\
& \{n i l\}_{\mathbb{D}} \times T \\
& =\text { "definition of } \llbracket \Phi \rrbracket " \\
& \llbracket \Phi \rrbracket
\end{aligned}
$$

Theorem 6.7 $(y \in A) \backslash i= \begin{cases}\bullet y \in A & \text { if } y \in i \\ y \in A & \text { if } y \notin i\end{cases}$

Proof Similar to that of Theorem 6.9 below

Theorem $6.8(\bullet y \in A) \backslash i=\bullet y \in A$

Proof Similar to that of Theorem 6.10 below $\square$

Theorem 6.9 $(y \in A, S) \backslash i= \begin{cases}\bullet y \in A,(S \backslash i) & \text { if } y \in i \\ y \in A,(S \backslash i) & \text { if } y \notin i\end{cases}$

Proof Given in Figure 6.2. After the third step, the proof proceeds by a two part case analysis for the case $y \in i$ and $y \notin i$.

Theorem $6.10(\bullet y \in A, S) \backslash i=\bullet y \in A,(S \backslash i)$.

\section{Proof}

$$
\llbracket(\bullet y \in A, S) \backslash i \rrbracket
$$

$=$ "Fact 6.20 "

$$
\left\{\llbracket \bullet y \in A, S \rrbracket_{\text {dom }} \| i\right\}_{\mathbb{\mathbb { D }}} \times \llbracket(\bullet y \in A, S) \backslash i \rrbracket_{L s g}
$$

$=$ "definitions of $\llbracket-\rrbracket_{d o m}$ and $\llbracket-\rrbracket_{L s g}$ "

$$
\left\{\left(\operatorname{inr}(y): \llbracket S \rrbracket_{d o m}\right) \backslash i\right\}_{\mathbb{D}} \times \sum y \in A . \llbracket S \rrbracket_{L s g}
$$

$=$ "definition of domain hiding"

$$
\left\{\operatorname{inr}(y):\left(\llbracket S \rrbracket_{d o m} \| i\right)\right\}_{\mathbb{D}} \times \sum y \in A . \llbracket S \rrbracket_{L s g}
$$

$=$ "definition of signature hiding, twice"

$$
\left\{i n r(y): \llbracket S \backslash i \rrbracket_{d o m}\right\}_{\mathbb{D}} \times \sum y \in A . \llbracket S \backslash i \rrbracket_{L s g}
$$

$=$ "definitions of $\llbracket \rrbracket_{d o m}$ and $\llbracket-\rrbracket_{L s g}$ "

$$
\left\{\llbracket \bullet y \in A,(S \backslash i) \rrbracket_{d o m}\right\}_{\mathbb{D}} \times \llbracket \bullet y \in A,(S \backslash i) \rrbracket_{L s g}
$$

$=$ "definition of $\llbracket-\rrbracket$ on signature terms"

$$
\llbracket \bullet y \in A,(S \backslash i) \rrbracket
$$




\section{Proof (of Theorem 6.9)}

$\llbracket(y \in A, S) \backslash i \rrbracket$

$=$ "Fact 6.20 "

$$
\begin{aligned}
& \left\{\llbracket(y \in A, S) \rrbracket_{d o m} \| i\right\}_{\mathbb{D}} \times \llbracket(y \in A, S) \backslash i \rrbracket_{L s g} \\
& =\text { "definition of } \llbracket-\rrbracket_{d o m} " \\
& \left\{\left(\text { inl }(y): \llbracket S \rrbracket_{d o m}\right) \rrbracket i\right\}_{\mathbb{D}} \times \llbracket y \in A, S \rrbracket_{L s g}
\end{aligned}
$$

\section{Case $y \in i$ :}

$$
\left\{\left(\operatorname{inl}(y): \llbracket S \rrbracket_{d o m}\right) \rrbracket i\right\}_{\mathbb{D}} \times \llbracket y \in A, S \rrbracket_{L s g}
$$

$=$ "assumption $y \in i$, definition of domain hiding, definition of $\llbracket-\rrbracket_{L s g}$ "

$$
\left\{\operatorname{inr}(y):\left(\llbracket S \rrbracket_{d o m} \| i\right)\right\}_{\mathbb{D}} \times \sum y \in A \cdot \llbracket S \rrbracket_{L s g}
$$

$=$ "definitions of domain hiding and signature hiding"

$$
\left\{\operatorname{inr}(y): \llbracket S \backslash i \rrbracket_{d o m}\right\}_{\mathbb{D}} \times \sum y \in A . \llbracket S \backslash i \rrbracket_{L s g}
$$

$=$ "definitions of $\llbracket-\rrbracket_{\text {dom }}$ and $\llbracket-\rrbracket_{L s g}$ "

$$
\left\{\llbracket \bullet y \in A,(S \backslash i) \rrbracket_{d o m}\right\}_{\mathbb{D}} \times \llbracket \bullet y \in A,(S \backslash i) \rrbracket_{L s g}
$$

$=$ "definition of $\llbracket-\rrbracket "$

$$
\llbracket \bullet y \in A,(S \backslash i) \rrbracket
$$

Case $y \notin i$ :

$$
\left\{\left(\operatorname{inl}(y): \llbracket S \rrbracket_{d o m}\right) \rrbracket i\right\}_{\mathbb{D}} \times \llbracket y \in A, S \rrbracket_{L s g}
$$

$=$ "assumption $y \notin i$, definition of domain hiding, definition of $\llbracket-\rrbracket_{L s g}$ " $\left\{\operatorname{inl}(y):\left(\llbracket S \rrbracket_{d o m} \| i\right)\right\}_{\mathbb{D}} \times \sum y \in A \cdot \llbracket S \rrbracket_{L s g}$

$=$ "definitions of domain hiding and signature hiding"

$$
\left\{\operatorname{inl}(y): \llbracket S \backslash i \rrbracket_{d o m}\right\}_{\mathbb{D}} \times \sum y \in A . \llbracket S \backslash i \rrbracket_{L s g}
$$

$=$ "definitions of $\llbracket-\rrbracket_{\text {dom }}$ and $\llbracket-\rrbracket_{L s g}$ "

$$
\left\{\llbracket y \in A,(S \backslash i) \rrbracket_{d o m}\right\}_{\mathbb{D}} \times \llbracket y \in A,(S \backslash i) \rrbracket_{L s g}
$$

$=$ "definition of $\llbracket-\rrbracket "$

$$
\llbracket y \in A,(S \backslash i) \rrbracket
$$

Figure 6.2: A proof of Theorem 6.9 
The properties of signature hiding are determined by those of the domain hiding function $\left(-\mathbb{N}_{-}\right)$. Lemmas $6.1-6.3$ give some properties of $\left(-\|_{-}\right)$which we will use to justify some laws of signature hiding; we state these lemmas without proof. Lemma 6.1 says that hiding an empty set of names leaves a domain unchanged; Lemma 6.2 says that two applications of $\left(-\mathbb{U}_{-}\right)$can be composed into a single application of $\left(-\mathbb{N}_{-}\right)$. Lemma 6.3 says that the order in which names are hidden is not important.

Lemma 6.1 $l \rrbracket\{\}=l$ where $l \in \mathbb{D}$.

Lemma 6.2 $(l \rrbracket i) \rrbracket j=l \rrbracket(i \cup j)$ where $l \in \mathbb{D}$ and $i, j \in \operatorname{Set}(\mathbb{S})$.

Lemma 6.3 $(l \rrbracket i) \rrbracket j=(l \rrbracket j) \rrbracket i$ where $l \in \mathbb{D}$ and $i, j \in \operatorname{Set}(\mathbb{S})$.

Theorem 6.11, below, says that hiding an empty set of components in a signature does not change the signature.

Theorem 6.11 Given $S \operatorname{sig}, S \backslash\{\}=S$. $\square$

Proof

$$
S \backslash\{\}
$$

$=$ "definition of signature hiding in MLT"

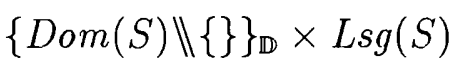

$=$ "Lemma 6.1"

$$
\{\operatorname{Dom}(S)\}_{\mathbb{D}} \times \operatorname{Lsg}(S)
$$

$=$ "semantics of signatures"

$S$

Two, or more, consecutive applications of signature hiding can be composed into a single application of signature hiding:

Theorem 6.12 $(S \backslash i) \backslash j=S \backslash(i \cup j)$ where $S$ sig and $i, j \in \operatorname{Set}(\mathbb{S})$.

Proof

$$
\begin{aligned}
&(S \backslash i) \backslash j \\
&= \text { "definition of signature hiding in MLT, twice" } \\
&\{(D o m(S) \backslash i) \backslash j\}_{\mathbb{D}} \times \operatorname{Lsg}(S) \text { "Lemma } 6.2 " \\
&\{D o m(S) \Downarrow(i \cup j)\}_{\mathbb{D}} \times \operatorname{Lsg}(S) \\
&=\text { "definition of signature hiding in MLT" } \\
& S \backslash(i \cup j)
\end{aligned}
$$

The order in which we compose consecutive applications of signature hiding does not affect the final result of the applications: 
Corollary 6.1 $\quad(S \backslash i) \backslash j=(S \backslash j) \backslash i$.

Proof Apply Theorem 6.12 to show that $(S \backslash i) \backslash j=S \backslash(i \cup j)$. Next, use set union commutativity to show that $S \backslash(i \cup j)=S \backslash(j \cup i)$. Then apply Theorem 6.12, to show that $S \backslash(j \cup i)=(S \backslash j) \backslash i$.

\subsection{Signature Concatenation}

In this section, we define the signature concatenation operator. An application of this operator has the form $S_{1} \oplus S_{2}$ for $S_{1}$ and $S_{2}$ signatures. $S_{1} \oplus S_{2}$ is a signature containing all the components defined in $S_{1}$ followed by all the components in $S_{2}$. The right-hand argument, $S_{2}$, may be dependent on $S_{1}$ (i.e. $S_{1} \vdash S_{2}$ sig). Consider the following signatures:

$$
\begin{aligned}
& S_{1}=S q r \in U_{1}, \text { size } \in S q r \rightarrow \mathbb{N} \\
& S_{2}=\text { area } \in S q r \rightarrow \mathbb{N} \text {, size } \in S q r \rightarrow \mathbb{N}
\end{aligned}
$$

Note that $S_{2}$ is dependent on the component $S q r$ in $S_{1}$. The result of the signature concatenation $S_{1} \oplus S_{2}$ is given below:

$$
S_{1} \oplus S_{2}=S q r \in U_{1}, \text { size } \in \text { Sqr } \rightarrow \mathbb{N} \text {, area } \in \text { Sqr } \rightarrow \mathbb{N} \text {, size } \in S q r \rightarrow \mathbb{N}
$$

Note that name size, which is used in both $S_{1}$ and $S_{2}$, is overloaded in $S_{1} \oplus S_{2}$. The signature concatenation operator overloads any name that appears in both its arguments. Overloading resolves any potential name-clashes arising in the result of a concatenation. We discuss the issue of name-clashes in more detail in Section 6.12.

\subsubsection{A Formal Definition of Signature Concatenation}

Two signatures are concatenated by concatenating their domains, using list concatenation (H), and concatenating their loose-signatures, using the loose-signature concatenation function. An application of loose signature concatenation has the form $P \otimes Q$ for $P$ and $Q$ loose signatures. $P \otimes Q$ is a loose signature containing all the components in $P$ followed by all the components in $Q$ (i.e. $P \otimes Q$ is $P$ with its final unit type $(T)$ replaced by $Q$ ). $Q$ may be dependent on the components given in $P$ (i.e. $P \vdash Q$ lsg). Loose signature concatenation is defined in Definition 6.11.

We illustrate the use of loose-signature concatenation by considering the loosesignatures of $S_{1}$ and $S_{2}$ above:

$$
\begin{aligned}
& \llbracket S_{1} \rrbracket_{L s g}=\sum S q r \in U_{1} . \sum \text { size } \in S q r \rightarrow \mathbb{N} . T \\
& \llbracket S_{2} \rrbracket_{L s g}=[\text { Sqr }][\text { size }] \sum \text { area } \in S q r \rightarrow \mathbb{N} . \sum \text { size } \in S q r \rightarrow \mathbb{N} . T
\end{aligned}
$$

The prefix $[S q r][s i z e]$ on $\llbracket S_{2} \rrbracket_{L s g}$ indicates that $\llbracket S_{2} \rrbracket_{L s g}$ is dependent on $\llbracket S_{1} \rrbracket_{L s g}$. The result of the loose-signature concatenation $\llbracket S_{1} \rrbracket_{L s g} \otimes \llbracket S_{2} \rrbracket_{L s g}$ is:

$$
\sum S q r \in U_{1} . \sum \text { size } \in S q r \rightarrow \mathbb{N} . \sum \text { area } \in S q r \rightarrow \mathbb{N} . \sum \text { size } \in S q r \rightarrow \mathbb{N} . T
$$


The free variable $S q r$ in $\llbracket S_{2} \rrbracket_{L s g}$ becomes bound to $S q r \in U_{1}$. The overloading of size is resolved by the usual scope rules for quantified expressions in MLT.

\section{Definition 6.11 (Loose Signature Concatenation $(\otimes)$ )}

Given any $P$ lsg, $\sum x \in A . B(x)$ lsg, and $\sum x \in A . B(x) \vdash Q$ lsg:

$$
\begin{array}{ll}
T \otimes P & =P \\
\left(\sum x \in A . B(x)\right) \otimes Q & =\sum x \in A .(B(x) \otimes Q(x))
\end{array}
$$

The formal definition of signature concatenation is given in Definition 6.12, and uses the notation defined in Definition 6.13 (recall the remark in Section 6.3.1). Note that in Definition 6.13, we write the right-hand argument of $\oplus$ as $\left(\{l\}_{\mathbb{D}} \times Q\right)$ so that we can refer to its domain $l$ and loose signature $Q$ : if the right-hand argument of $\oplus$ is a dependent signature then we cannot apply Dom and $L s g$ to it, as Dom and Lsg are not defined on dependent signatures.

\section{Definition 6.12 (Signature Concatenation)}

Given $\llbracket S_{1} \rrbracket$ sig and $\llbracket S_{1} \rrbracket \vdash \llbracket S_{2} \rrbracket$ sig then

$$
\llbracket S_{1} \oplus S_{2} \rrbracket=\llbracket S_{1} \rrbracket \oplus \llbracket S_{2} \rrbracket
$$

\section{Definition 6.13 (Notation for Signature Concatenation in MLT $(\oplus)$ )}

Given any $S_{1}$ sig, and $S_{1} \vdash(\{l\} \times Q)$ sig where $l \in \mathbb{D}$ and $L s g\left(S_{1}\right) \vdash Q$ lsg:

$$
\begin{aligned}
& \operatorname{Dom}\left(S_{1} \oplus(\{l\} \times Q)\right)=\operatorname{Dom}\left(S_{1}\right)+l \\
& \operatorname{Lsg}\left(S_{1} \oplus(\{l\} \times Q)\right)=\operatorname{Lsg}\left(S_{1}\right) \otimes Q
\end{aligned}
$$

Given any signature concatenation $S_{1} \oplus S_{2}$ such that $S_{2}$ is not a dependent signature, then we can apply $L s g$ and Dom to $S_{2}$ to get the following facts:

Fact 6.11 If $S_{1}$ sig and $S_{2}$ sig then $\operatorname{Dom}\left(S_{1} \oplus S_{2}\right)=\operatorname{Dom}\left(S_{1}\right)+\operatorname{Dom}\left(S_{2}\right) \square$

Fact 6.12 If $S_{1}$ sig and $S_{2}$ sig then $\operatorname{Lsg}\left(S_{1} \oplus S_{2}\right)=\operatorname{Lsg}\left(S_{1}\right) \otimes \operatorname{Lsg}\left(S_{2}\right)$

\subsubsection{Laws of Signature Concatenation}

The properties of signature concatenation are determined by those of list concatenation and loose-signature concatenations. Lemmas 6.4 and 6.5 give some properties of loose-signature concatenation which we will use to justify some laws of signature concatenation. Lemma 6.4 says that the unit type is a two sided identity for $(\otimes)$, and Lemma 6.5 says that $(\otimes)$ is associative.

Lemma 6.4 $T \otimes P=P$ and $P \otimes T=P$ where $P$ lsg and $T$ is the unit type $\square$ Proof Trivial, and is omitted 
Lemma 6.5 $P \otimes(Q \otimes R)=(P \otimes Q) \otimes R$ where $P \operatorname{lsg}, Q \operatorname{lsg}$ and $R$ lsg $\square$ Proof Omitted $\square$

The empty signature $\Phi$ is a right- and left-identity for signature concatenation:

Theorem 6.13 $S \oplus \Phi=S$

\section{Proof}

$$
\begin{gathered}
\llbracket S \oplus \Phi \rrbracket \\
=\text { "definition of } \oplus, \text { definition of } \Phi " \\
\llbracket S \rrbracket \oplus\left(\{\text { nil }\}_{\mathbb{D}} \times T\right) \\
=\text { "definition of signature concatenation" } \\
\quad\{\text { Dom }(\llbracket S \rrbracket)+n i l\}_{\mathbb{D}} \times(L s g(\llbracket S \rrbracket) \otimes T) \\
=\text { "list calculus, Lemma } 6.4 " \\
\quad\{\text { Dom }(\llbracket S \rrbracket)\}_{\mathbb{D}} \times L s g(\llbracket S \rrbracket) \\
=\text { "definition of signature" } \\
\llbracket S \rrbracket
\end{gathered}
$$

Theorem 6.14 $\Phi \oplus S=S$

Proof Similar to that of Theorem 6.13 above $\square$

Theorems 6.15-6.18 (below) are a collection of laws that can be used to unfold applications of signature concatenation to canonical signatures.

Theorem 6.15 $(y \in A) \oplus S_{2}= \begin{cases}y \in A & \text { if } S_{2}=\Phi \\ y \in A, S_{2} & \text { if } S_{2} \neq \Phi\end{cases}$

Proof Similar to that of Theorem 6.17 below $\square$

Theorem 6.16 $(\bullet y \in A) \oplus S_{2}= \begin{cases}\bullet y \in A & \text { if } S_{2}=\Phi \\ \bullet y \in A, S_{2} & \text { if } S_{2} \neq \Phi\end{cases}$

Proof Similar to that of Theorem 6.17 below $\square$

Theorem 6.17 $\left(y \in A, S_{1}\right) \oplus S_{2}=y \in A,\left(S_{1} \oplus S_{2}\right)$ 


\section{Proof}

$$
\begin{aligned}
& \llbracket\left(y \in A, S_{1}\right) \oplus S_{2} \rrbracket \\
& =\text { "Fact 6.21" } \\
& \left\{\llbracket y \in A, S_{1} \rrbracket_{d o m} \# \llbracket S_{2} \rrbracket_{d o m}\right\}_{\mathbb{D}} \times\left(\llbracket y \in A, S_{1} \rrbracket_{L s g} \otimes \llbracket S_{2} \rrbracket_{L s g}\right) \\
& =\text { "definitions of } \llbracket-\rrbracket_{d o m} \text { and } \llbracket-\rrbracket_{L s g} \text { " } \\
& \left\{i n l(y):\left(\llbracket S_{1} \rrbracket_{d o m} \# \llbracket S_{2} \rrbracket_{d o m}\right)\right\}_{\mathbb{D}} \times\left(\left(\sum y \in A . \llbracket S_{1} \rrbracket_{L s g}\right) \otimes \llbracket S_{2} \rrbracket_{L s g}\right) \\
& =\text { "definition of } \oplus \text {, definition of } \otimes \text { " } \\
& \left\{\operatorname{inl}(y): \llbracket S_{1} \oplus S_{2} \rrbracket_{d o m}\right\}_{\mathbb{D}} \times\left(\sum y \in A .\left(\llbracket S_{1} \rrbracket_{L s g} \otimes \llbracket S_{2} \rrbracket_{L s g}\right)\right) \\
& =\text { "definitions of } \llbracket-\rrbracket_{d o m} \text { and } \llbracket-\rrbracket_{L s g} \text { " } \\
& \left\{\llbracket y \in A,\left(S_{1} \oplus S_{2}\right) \rrbracket_{d o m}\right\}_{\mathbb{D}} \times \llbracket y \in A,\left(S_{1} \oplus S_{2}\right) \rrbracket_{L s g} \\
& =\text { "definition of } \llbracket-\rrbracket \text { on signature terms" } \\
& \llbracket y \in A,\left(S_{1} \oplus S_{2}\right) \rrbracket
\end{aligned}
$$

Theorem $6.18\left(\bullet y \in A, S_{1}\right) \oplus S_{2}=\bullet y \in A,\left(S_{1} \oplus S_{2}\right)$

Proof Similar to that of Theorem 6.17 above $\square$

Signature concatenation is not commutative; this is because list concatenation and loose-signature concatenation are not commutative. However, signature concatenation is associative:

\section{Theorem 6.19 ( $\oplus$ associativity)}

If $S_{1}$ sig, $S_{2}$ sig and $S_{3}$ sig then $S_{1} \oplus\left(S_{2} \oplus S_{3}\right)=\left(S_{1} \oplus S_{2}\right) \oplus S_{3}$.

\section{Proof}

$$
S_{1} \oplus\left(S_{2} \oplus S_{3}\right)
$$

$=$ "Fact 6.11 , Fact 6.12 "

$$
S_{1} \oplus\left(\left\{\operatorname{Dom}\left(S_{2}\right)+\operatorname{Dom}\left(S_{3}\right)\right\}_{\mathbb{D}} \times\left(\operatorname{Lsg}\left(S_{2}\right) \otimes L s g\left(S_{3}\right)\right)\right)
$$

$=$ "definition of signature concatenation"

$$
\left\{\operatorname{Dom}\left(S_{1}\right)+\left(\operatorname{Dom}\left(S_{2}\right)+\operatorname{Dom}\left(S_{3}\right)\right)\right\}_{\mathbb{D}} \times\left(\operatorname{Lsg}\left(S_{1}\right) \otimes\left(\operatorname{Lsg}\left(S_{2}\right) \otimes L s g\left(S_{3}\right)\right)\right)
$$

$=$ "associativity of $\#$, associativity of $\otimes$ (Lemma 6.5)"

$$
\left\{\left(\operatorname{Dom}\left(S_{1}\right)+\operatorname{Dom}\left(S_{2}\right)\right)+\operatorname{Dom}\left(S_{3}\right)\right\}_{\mathbb{D}} \times\left(\left(L s g\left(S_{1}\right) \otimes L s g\left(S_{2}\right)\right) \otimes L s g\left(S_{3}\right)\right)
$$

$=$ "definition of signature concatenation"

$$
\left(\left\{\operatorname{Dom}\left(S_{1}\right)+\operatorname{Dom}\left(S_{2}\right)\right\}_{\mathbb{D}} \times\left(\operatorname{Lsg}\left(S_{1}\right) \otimes \operatorname{Lsg}\left(S_{2}\right)\right)\right) \oplus S_{3}
$$

$=$ "Fact 6.11 , Fact 6.12 "

$$
\left(S_{1} \oplus S_{2}\right) \oplus S_{3}
$$




\subsection{Translating Signature Operators}

In this section, we summarise the syntax of the signature operations defined in this chapter, and give some useful facts about the operations. The grammar sigops, in Definition 6.14, gives the syntax of signature operators; token $S$ (possibly subscripted) stands for sigops terms, and token $S l$ (possibly subscripted) stands for siglist terms. sigops is an extension of the grammar sig for canonical signatures. We assume that sigops is added to the syntax of types (i.e. Type ::= sigops).

Definition 6.14 (Syntax of Signature Operators)

$$
\begin{aligned}
& \text { sigops }::=\Phi|S l| S[p \backslash q]|S \backslash i| S_{1} \oplus S_{2} \\
& \text { siglist }::=y \in A|\bullet y \in A| y \in A, S l|\bullet y \in A, S l| S l[p \backslash q]|S l \backslash i| S l_{1} \oplus S l_{2}
\end{aligned}
$$

We obtain the following facts from the definition of the translation mapping on canonical signatures, which is $\llbracket S \rrbracket=\left\{\llbracket S \rrbracket_{\text {dom }}\right\}_{\mathbb{D}} \times \llbracket S \rrbracket_{L s g}$ for any $S$ : sig; recall that $\llbracket-\rrbracket_{\text {dom }}$ and 【-』Lsg translate canonical signatures into their domains and loose-signatures, respectively. Recall, from Chapter 5, that the semantic function $\llbracket-\rrbracket_{I d}$ translates an identifier token into a component name of type $\mathbb{S}$. The term $\llbracket i \rrbracket$ is the set of component names obtained by translating the term $i:$ Exp into MLT.

Fact $6.13 \llbracket\left[S[p \backslash q] \rrbracket_{\text {dom }}=\llbracket S \rrbracket_{\text {dom }}\left[\llbracket p \rrbracket_{I d} \rrbracket \llbracket q \rrbracket_{I d}\right] \square\right.$

Fact $6.14 \llbracket S \backslash i \rrbracket_{\text {dom }}=\llbracket S \rrbracket_{\text {dom }} \| \llbracket i \rrbracket \square$

Fact 6.15 $\llbracket S_{1} \oplus S_{2} \rrbracket_{d o m}=\llbracket S_{1} \rrbracket_{d o m}+\llbracket S_{2} \rrbracket_{d o m} \square$

Fact 6.16 $\llbracket S[p \backslash q] \rrbracket_{L s g}=\llbracket S \rrbracket_{L s g} \square$

Fact $6.17 \llbracket S \backslash i \rrbracket_{L s g}=\llbracket S \rrbracket_{L s g} \square$

Fact 6.18 $\llbracket S_{1} \oplus S_{2} \rrbracket_{L s g}=\llbracket S_{1} \rrbracket_{L s g} \otimes \llbracket S_{2} \rrbracket_{L s g}$

Fact $6.19 \llbracket S[p \backslash q] \rrbracket=\left\{\llbracket S \rrbracket_{\text {dom }}\left[\llbracket p \rrbracket_{I d} \rrbracket \llbracket \llbracket q \rrbracket_{I d}\right]\right\}_{\mathbb{D}} \times \llbracket S \rrbracket_{L s g}$

Fact $6.20 \llbracket S \backslash i \rrbracket=\left\{\llbracket S \rrbracket_{d o m} \rrbracket \llbracket i \rrbracket\right\}_{\mathbb{D}} \times \llbracket S \rrbracket_{L s g} \square$

Fact 6.21 $\llbracket S_{1} \oplus S_{2} \rrbracket=\left\{\llbracket S_{1} \rrbracket_{d o m}+\llbracket S_{2} \rrbracket_{\text {dom }}\right\}_{\mathbb{D}} \times\left(\llbracket S_{1} \rrbracket_{L s g} \otimes \llbracket S_{2} \rrbracket_{L s g}\right) \square$

\subsection{Computational Element Renaming}

In this section, we define the computational element renaming operator. An application of this operator has the form $m[p \backslash q]$ for $p$ and $q$ names and $m$ a computational element. $m[p \backslash q]$ is $m$ with component $p$ renamed to $q$. There is a close relationship between computational element renaming and signature renaming: given any signature $S$ and any $m \in S$ then $m[p \backslash q] \in S[p \backslash q]$. 
We give an example of computational element renaming by considering the following signature $S_{1}$ and computational element $m_{1} \in S$ :

$$
\begin{aligned}
S_{1}= & \text { Book } \in U_{1}, \text { Stock } \in U_{1}, \text { add } \in \text { Book } \rightarrow \text { Stock } \rightarrow \text { Stock } \\
m_{1}= & \text { module } \\
& \text { Book }=\mathbb{N}, \\
& \text { Stock }=\text { Set }(\text { Book }), \\
& \text { add }=\lambda b \in \text { Book. } \lambda \text { s } \in \text { Stock. } s \cup\{b\} \\
& \text { end } \in S_{1}
\end{aligned}
$$

$m_{1}\left[B o o k \backslash\right.$ Video] is $m_{1}$ with the component Book renamed to Video. $m_{1}[B o o k \backslash V i d e o]$ has the signature $S_{1}[B o o k \backslash V i d e o]$, and its canonical form is:

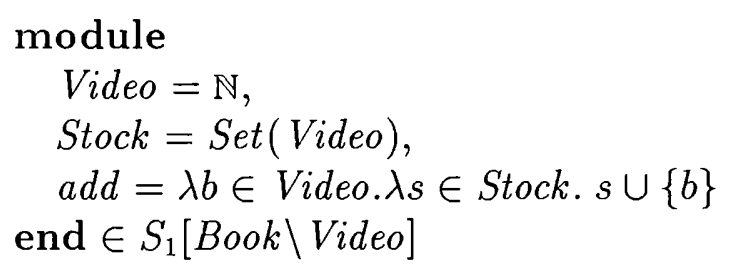

The properties of computational element renaming are similar to those of signature renaming. For example, an attempt to rename a non-existent component leaves a computational element unchanged; and an attempt to rename any overloaded component name, $p$ for example, in any computational element, $m$ for example, only renames the first version of $p$ in $m$.

\subsubsection{A Definition of Computational Element Renaming}

We give a formal definition of computational element renaming in Definition 6.15 with explanation following. Note that Definition 6.15 uses the notation given in Definition 6.16 (recall the remark in Section 6.3.1). We use the domain substitution function _[- $\mathbb{I}_{-}$(given previously in Definition 6.7) to substitute the name $p$ by $q$ in the domain of $m$. Computational element renaming does not alter the value tuple of a computational element. Note that in Definition 6.16 , we also state that given $m \in S$, then $m[p \backslash q] \in S[p \backslash q]$; a proof of this fact is given in Figure 6.3.

\section{Definition 6.15 (Computational Element Renaming)}

Given $\llbracket S \rrbracket$ sig, a computational element $\llbracket m \rrbracket \in \llbracket S \rrbracket$ and $\llbracket p \rrbracket$, $\llbracket q \in \mathbb{S}$, then:

$$
\llbracket m[p \backslash q] \rrbracket=\llbracket m \rrbracket[\llbracket p \rrbracket \backslash \llbracket q \rrbracket] \in \llbracket S[p \backslash q] \rrbracket
$$

\section{Definition 6.16 (Computational Element Renaming in MLT)}

Given $S$ sig, a computational element $m \in S$ and $p, q \in \mathbb{S}$, then:

$$
m[p \backslash q]=\langle\operatorname{dom}(m)[p \Downarrow q], \operatorname{val}(m)\rangle \in S[p \backslash q]
$$




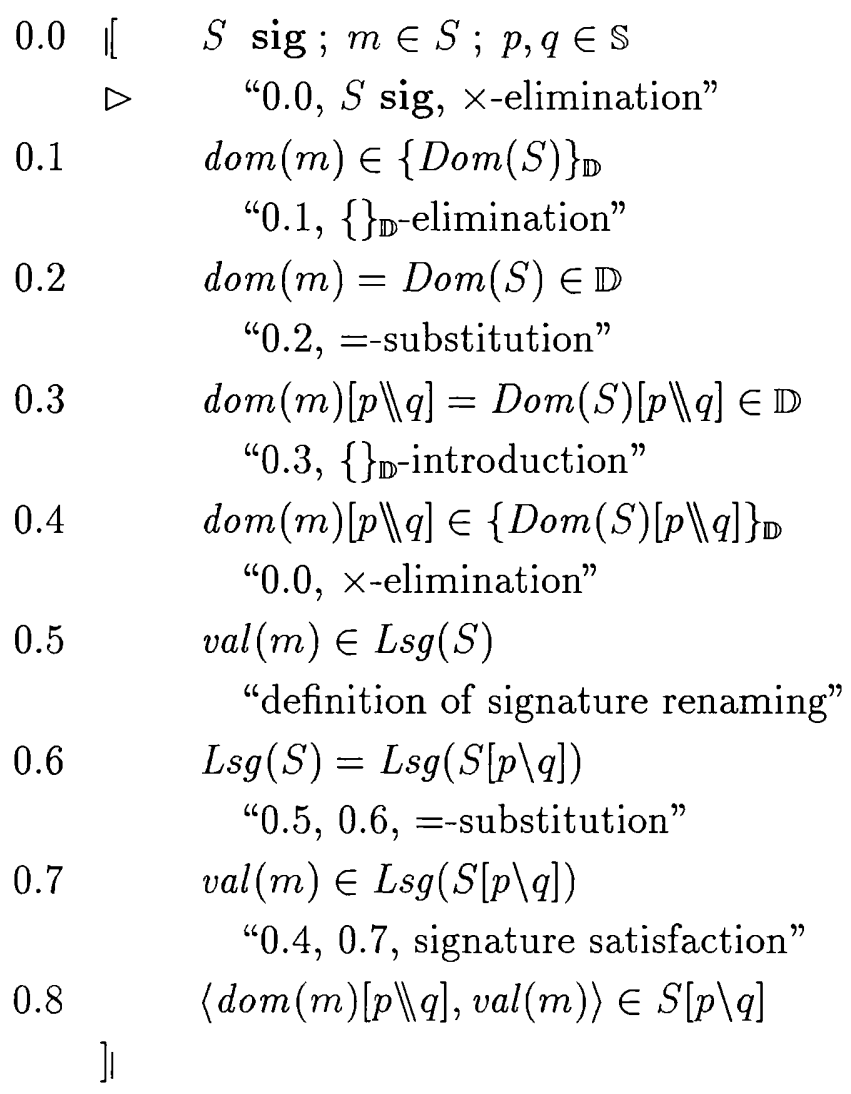

Figure 6.3: Proof of the Type Correctness of Computational Element Renaming

\subsubsection{Laws of Computational Element Renaming}

Theorems 6.20-6.24 (below) give laws for renaming canonical computational elements. Note the similarity, in function, of these laws to the laws for renaming canonical signatures, given in Section 6.3.2. Note that Theorems 6.23 and 6.24 use computational element concatenation $(\oplus)$ which will be defined in Section 6.9: the concatenation of two computational elements $m_{1}$ and $m_{2}$ (written as $m_{1} \oplus m_{2}$ ) is a computational element containing the components of $m_{1}$ followed by the components of $m_{2}$.

Theorem 6.20 module end $[p \backslash q]=$ module end $\square$

\section{Proof Omitted $\square$}

Theorem 6.21 module $y=e$ end $[p \backslash q]=\left\{\begin{array}{l}\operatorname{module} y=e \text { end if } p \neq y \\ \text { module } q=e \text { end if } p=y\end{array}\right.$

Proof Given in Figure 6.4 口

Theorem 6.22 module $\bullet y=e$ end $[p \backslash q]=\left\{\begin{array}{l}\operatorname{module} \bullet y=e \text { end } \quad \text { if } p \neq y \\ \operatorname{module} \bullet q=e \text { end } \quad \text { if } p=y\end{array}\right.$

Proof Similar to that of Theorem 6.21 above $\square$ 
Proof (of Theorem 6.21)

$\llbracket \operatorname{module} y=e$ end $[p \backslash q] \rrbracket$

$=$ "Fact 6.32"

【module $y=e$ end $\rrbracket[\llbracket p \rrbracket \backslash \llbracket q \rrbracket]$

= "definition of computational element renaming"

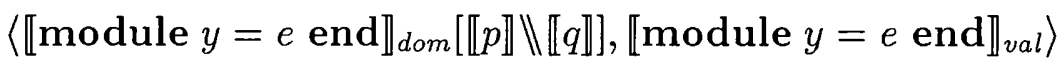

$=$ "definitions of $\llbracket-\rrbracket_{\text {dom }}$ and $\llbracket-\rrbracket_{\text {val }}$ "

$\langle[\operatorname{inl} l(\llbracket y \rrbracket)] \llbracket[p \rrbracket \rrbracket \llbracket q \rrbracket],\langle\llbracket e \rrbracket, t t\rangle\rangle$

Case $p=y$ :

$\langle[\operatorname{inl} l(\llbracket y \rrbracket)][\llbracket p \rrbracket \rrbracket \llbracket q \rrbracket],\langle\llbracket e \rrbracket, t t\rangle\rangle$

$=$ "assumption $p=y$, definition of domain substitution"

$\langle[\operatorname{inl}(\llbracket q \rrbracket)],\langle\llbracket e \rrbracket, t t\rangle\rangle$

$=$ "definitions of $\llbracket-\rrbracket_{\text {dom }}$ and $\llbracket-\rrbracket_{v a l}$ "

$\left\langle\llbracket\right.$ module $q=e$ end $\rrbracket_{\text {dom }}, \llbracket$ module $q=e$ end $\left.\rrbracket_{\text {val }}\right\rangle$

$=$ "definition of the translation mapping on computational elements"

$\llbracket$ module $q=e$ end $\rrbracket$

Case $p \neq y$ :

$\langle[\operatorname{inl} l(\llbracket y \rrbracket)] \llbracket[p \rrbracket \rrbracket \llbracket q \rrbracket],\langle\llbracket e \rrbracket, t t\rangle\rangle$

$=$ "assumption $p=y$, definition of domain substitution"

$\langle\llbracket \operatorname{inl} l(\llbracket y \rrbracket)],\langle\llbracket e \rrbracket, t t\rangle\rangle$

$=$ "definitions of $\mathbb{\llbracket}-\rrbracket_{\text {dom }}$ and $\llbracket-\rrbracket_{v a l}$ "

$\left\langle\llbracket \operatorname{module} y=e\right.$ end $\rrbracket_{\text {dom }}, \llbracket \operatorname{module} y=e$ end $\left.\rrbracket_{\text {val }}\right\rangle$

$=$ "definition of the translation mapping on computational elements"

$\llbracket \operatorname{module} y=e$ end $\rrbracket$

Figure 6.4: A proof of Theorem 6.21

Theorem 6.23 module $y=e, b$ end $[p \backslash q]$

$= \begin{cases}\text { module } y=e \text { end } \oplus \text { module } b(y \backslash e) \text { end }[p \backslash q] & \text { if } p \neq y \\ \text { module } q=e, b(p \backslash q) \text { end } & \text { if } p=y\end{cases}$

Proof Similar to that of Theorem 6.21 above $\square$

Theorem 6.24 module $\bullet y=e, b$ end $[p \backslash q]$

$$
= \begin{cases}\text { module } \bullet y=e \text { end } \oplus \text { module } b(y \backslash e) \text { end }[p \backslash q] & \text { if } p \neq y \\ \text { module } \bullet q=e, b(p \backslash q) \text { end } & \text { if } p=y\end{cases}
$$

Proof Similar to that of Theorem 6.21 above $\square$ 


\subsection{Computational Element Hiding}

In this section, we define the computational element hiding operator. An application of this operator has the form $m \backslash i$ for $m$ any computational element and $i$ a set of component names. $m \backslash i$ is $m$ with all visible components named in $i$ redefined as local components. Given any signature $S$, and any $m \in S$, then $m \backslash i \in S \backslash i$.

We give an example application of computational element hiding by considering the following signature $S_{1}$ and computational element $m_{1} \in S_{1}$ :

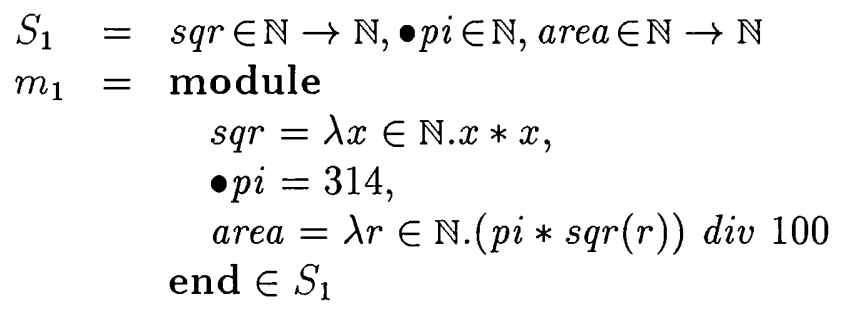

$m_{1} \backslash\{s q r, p i\}$ is $m_{1}$ with the components $s q r$ and $p i$ made local. The signature of $m_{1} \backslash\{s q r, p i\}$ is $S_{1} \backslash\{s q r, p i\}$. The canonical form of $m_{1} \backslash\{s q r, p i\}$ is:

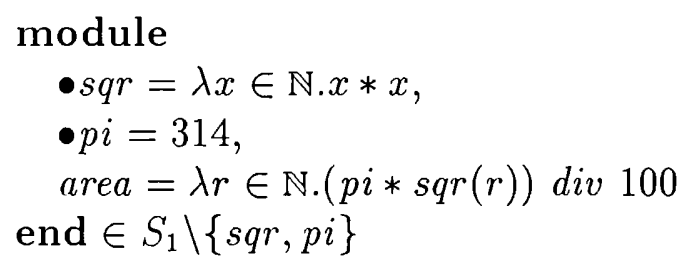

The properties of computational element hiding are similar to those of signature hiding. For example, an attempt to hide a non-existent component leaves a computational element unchanged; and if a component name, $p$ for example, is overloaded in a computational element $m$ then $m \backslash\{p\}$ hides all the components named $p$ in $m$.

\subsubsection{A Definition of Computational Element Hiding}

We give a formal definition of computational element hiding in Definition 6.17 with explanation following. Note that Definition 6.17 uses the notation given in Definition 6.18 (recall the remark in Section 6.3.1). We use the domain hiding function $-\mathbb{N}_{-}$ (given previously in Definition 6.10) to tag, with inr, all names in the domain of $m$ that also appear in $i$. Computational element hiding does not alter the value tuple of a computational element; so $\operatorname{val}(\mathrm{m})$ remains unchanged. Note that in Definition 6.18, we state that $m \backslash i \in S \backslash i$; we omit the proof of this property as it is similar, in style, to the proof of the type correctness of computational element renaming.

Definition 6.17 (Computational Element Hiding)

Given $\llbracket S \rrbracket$ sig, any computational element $\llbracket m \rrbracket \in \llbracket S \rrbracket$ and $\llbracket i \rrbracket \in S e t(\mathbb{S})$, then:

$$
\llbracket m \backslash i \rrbracket=\llbracket m \rrbracket \backslash \llbracket i \rrbracket \in \llbracket S \backslash i \rrbracket
$$


Definition 6.18 (Computational Element Hiding in MLT)

Given $S$ sig, any computational element $m \in S$ and $i \in \operatorname{Set}(\mathbb{S})$, then: $m \backslash i=\langle\operatorname{dom}(m) \rrbracket i, \operatorname{val}(m)\rangle \in S \backslash i$

\subsubsection{Laws of Computational Element Hiding}

Given any canonical computational element $m, m \backslash i(i \in \operatorname{Set}(\mathbb{S}))$ can be rewritten by putting bullets in front of all visible components in $m$ which are also named in $i$. This property is justified by Theorems $6.25-6.29$ below. Note that Theorems 6.28 and 6.29 use the computational element concatenation operator $(\oplus)$ which will be defined in Section 6.9.

Theorem 6.25 module end $\backslash i=$ module end

Proof Omitted $\square$

Theorem 6.26 module $y=e$ end $\backslash i= \begin{cases}\text { module } y=e \text { end } & \text { if } y \notin i \\ \operatorname{module} \bullet y=e \text { end } & \text { if } y \in i\end{cases}$

Proof Omitted $\square$

Theorem 6.27 module $\bullet y=e$ end $\backslash i=$ module $\bullet y=e$ end

Proof Omitted $\square$

Theorem 6.28 module $y=e, b$ end $\backslash i$ $= \begin{cases}\text { module } y=e \text { end } \oplus \text { module } b(y \backslash e) \text { end } \backslash i & \text { if } y \notin i \\ \text { module } \bullet y=e \text { end } \oplus \text { module } b(y \backslash e) \text { end } \backslash i & \text { if } y \in i\end{cases}$

Proof Omitted $\square$

Theorem 6.29 module $\bullet y=e, b$ end $\backslash i$ $=$ module $\bullet y=e$ end $\oplus$ module $b(y \backslash e)$ end $\backslash i$

\section{Proof Omitted $\square$}

Hiding an empty set of components in a computational element does not change the computational element:

Theorem 6.30 If $S$ sig and $m \in S$ then $m \backslash\{\}=m \in S$

Proof Omitted $\square$ 
Two consecutive applications of computational element hiding can be composed into a single application:

Theorem 6.31 If $S \operatorname{sig}, m \in S$ and $i, j \in \operatorname{Set}(\mathbb{S})$ then $m \backslash i \backslash j=m \backslash(i \cup j) \in S \backslash(i \cup j)$

\section{Proof Omitted $\square$}

The order in which consecutive applications of computational element hiding are made does not affect the final result of the applications:

Theorem 6.32 If $S$ sig, $m \in S$ and $i, j \in S e t(\mathbb{S})$ then $m \backslash i \backslash j=m \backslash j \backslash i \in S \backslash j \backslash i$

Proof Omitted $\square$

\subsection{Computational Element Concatenation}

In this section, we define the computational element concatenation operator. An application of this operator has the form $m_{1} \oplus m_{2}$ for $m_{1}$ and $m_{2}$ any computational elements. $m_{1} \oplus m_{2}$ is a computational element containing all the components in $m_{1}$ followed by all the components in $m_{2}$. Given any signatures $S_{1}$ and $S_{2}$, and computational elements $m_{1} \in S_{1}$ and $m_{2} \in S_{2}$, then $m_{1} \oplus m_{2} \in S_{1} \oplus S_{2}$.

We give an example of computational element concatenation by considering the following signatures and computational elements:

$$
\begin{aligned}
& S_{1}=\text { Sqr } \in U_{1}, \text { size } \in \text { Rect } \rightarrow \mathbb{N} \\
& S_{2}=\text { area } \in \mathbb{N} \rightarrow \mathbb{N} \text {, size } \in \text { Rect } \rightarrow \mathbb{N} \\
& m_{1}=\text { module } S q r=\mathbb{N} \text {, size }=\lambda s \in \mathbb{N} . s \text { end } \in S_{1} \\
& m_{2}=\text { module } \text { area }=\lambda s \in \mathbb{N} . s * s, \text { size }=\lambda s \in \mathbb{N} . s * 2 \text { end } \in S_{2}
\end{aligned}
$$

The result of the concatenating $m_{1}$ and $m_{2}$ is given below:

$$
\begin{aligned}
m_{1} \oplus m_{2}=\text { module } & \\
\text { Sqr } & =\mathbb{N}, \\
\text { size } & =\lambda s \in \mathbb{N} . s, \\
\text { area } & =\lambda s \in \mathbb{N} . s * s, \\
\text { size } & =\lambda s \in \mathbb{N} . s * 2 \\
\text { end } \in & S_{1} \oplus S_{2}
\end{aligned}
$$

Like signature concatenation, the computational element concatenation operator resolves potential name-clashes by overloading any component name used by both its arguments. For example, the name size which appears in $m_{1}$ and $m_{2}$ (above) is overloaded in $m_{1} \oplus m_{2}$. 


\subsubsection{Definition of Computational Element Concatenation}

We concatenate two computational elements by concatenating their domains, and by concatenating their value tuples. Domains are concatenated using list concatenation. Value tuples are concatenated using tuple concatenation which is described below.

An application of tuple concatenation has the form $p \odot q$ for $p$ and $q$ any value tuples. The result of $p \odot q$ is a value tuple containing all the components in $p$ followed by all the components in $q$. In other words, $p \odot q$ is $p$ with its final component $t t \in T$ replaced by $q$. For example, given two loose signatures $P$ and $Q$, and two value tuples,

$$
\begin{aligned}
& p=\left\langle e_{1},\left\langle\ldots,\left\langle e_{i}, t t\right\rangle \ldots\right\rangle\right\rangle \in P \text { and } \\
& q=\left\langle e_{j},\left\langle\ldots,\left\langle e_{n}, t t\right\rangle \ldots\right\rangle\right\rangle \in Q,
\end{aligned}
$$

then

$$
p \odot q=\left\langle e_{1}, \ldots,\left\langle e_{i},\left\langle e_{j},\left\langle\ldots,\left\langle e_{n}, t t\right\rangle \ldots\right\rangle\right\rangle\right\rangle \ldots\right\rangle \in P \otimes Q .
$$

We give a formal definition of tuple concatenation in Definition 6.19. Note that we use the loose signature concatenation operator $(\otimes)$ to give the type of a tuple concatenation.

Definition 6.19 (Tuple concatenation $(\odot)$ )

Given $\sum x \in A . B(x)$ lsg, $Q$ lsg, $\langle a, b\rangle \in \sum x \in A . B(x)$ and $q \in Q$, then:

$$
\begin{array}{lll}
t t \odot q & =q & \in T \otimes Q=Q \\
\langle a, b\rangle \odot q & =\langle a, b \odot q\rangle & \in\left(\sum x \in A . B(x)\right) \otimes Q=\sum x \in A .(B(x) \otimes Q)
\end{array}
$$

Fact 6.22 Given $P$ lsg, $Q$ lsg, $p \in P$ and $q \in Q$, then $p \odot q \in P \otimes Q$

We give a formal definition of computational element concatenation $(\oplus)$ in Definition 6.20, with an explanation following. Note that Definition 6.20 uses the notation given in Definition 6.21 (recall the remark in Section 6.3.1). We concatenate the domains of $m_{1}$ and $m_{2}$ using list concatenation $(H)$. We use tuple concatenation $(\odot)$ to concatenate the value tuples of $m_{1}$ and $m_{2}$. Note that $m_{1} \oplus m_{2} \in S_{1} \oplus S_{2}$; we give a proof of this property in Figure 6.5.

\section{Definition 6.20 (Computational Element Concatenation)}

Given any $\llbracket S_{1} \rrbracket \operatorname{sig}, \llbracket S_{2} \rrbracket \operatorname{sig}, \llbracket m_{1} \rrbracket \in \llbracket S_{1} \rrbracket$ and $\llbracket m_{2} \rrbracket \in \llbracket S_{2} \rrbracket$, then

$$
\llbracket m_{1} \oplus m_{2} \rrbracket=\llbracket m_{1} \rrbracket \oplus \llbracket m_{1} \rrbracket \in \llbracket S_{1} \oplus S_{2} \rrbracket
$$

\section{Definition 6.21 (Computational Element Concatenation in MLT $(\oplus)$ )}

Given any $S_{1} \operatorname{sig}, S_{2}$ sig, $m_{1} \in S_{1}$ and $m_{2} \in S_{2}$, then

$$
m_{1} \oplus m_{2}=\left\langle\operatorname{dom}\left(m_{1}\right)+\operatorname{dom}\left(m_{2}\right), \operatorname{val}\left(m_{1}\right) \odot \operatorname{val}\left(m_{2}\right)\right\rangle \in S_{1} \oplus S_{2}
$$




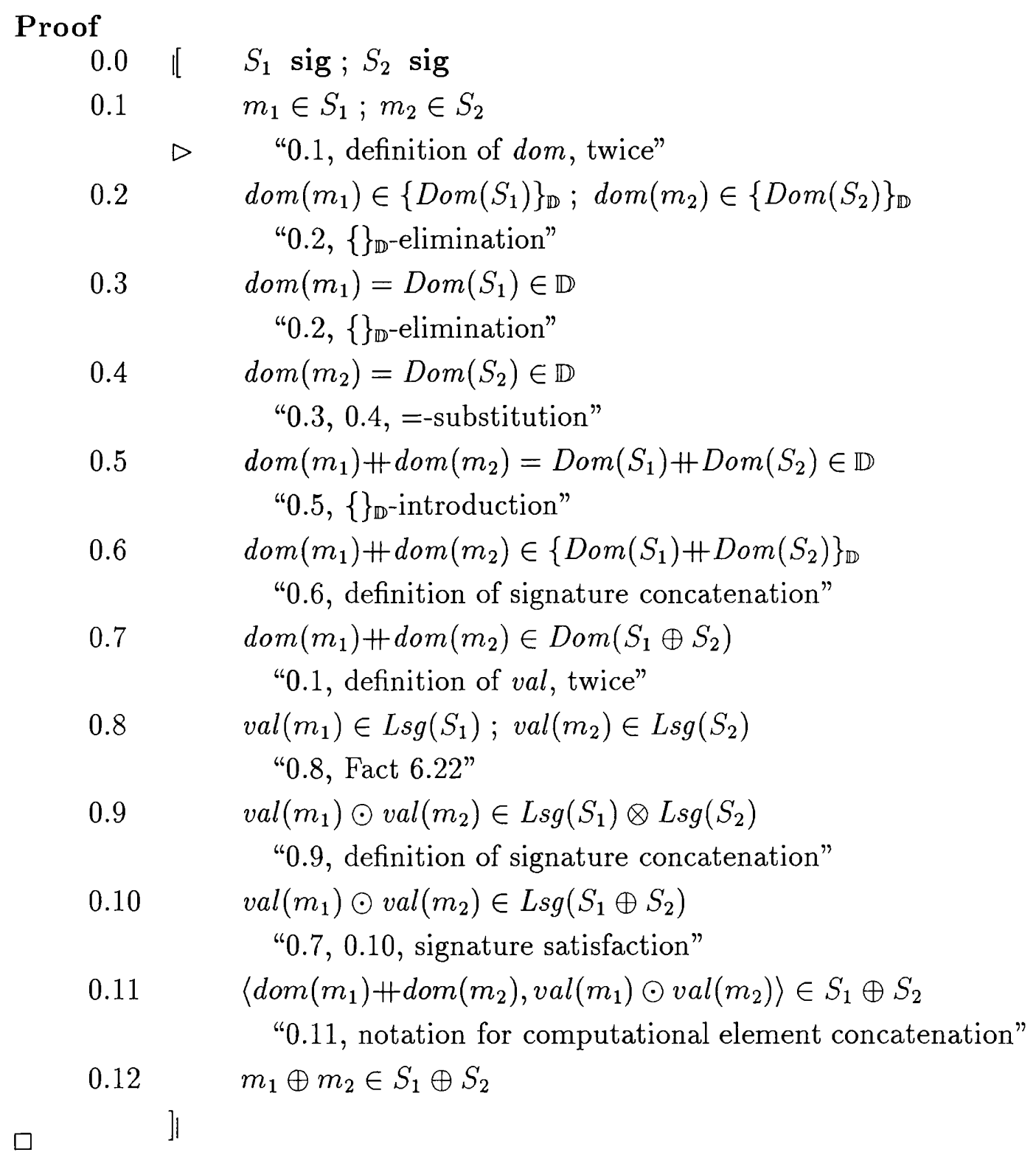

Figure 6.5: Proof of the Type Correctness of Computational Element Concatenation 


\subsubsection{Laws of Computational Element Concatenation}

Theorem 6.33 is a law of $\oplus$ for the special case where the signature of the right-hand argument of $\oplus$ depends on the left-hand argument of $\oplus$. Note that in Theorem 6.33, we express the signature $S_{2}\left(\mid m_{1}\right)_{S_{1}}$ using the signature instantiation notation defined in Section 6.2.2: $S_{2}\left(\mid m_{1}\right)_{S_{1}}$ is a non-dependent signature obtained by replacing each free occurrence of a component name in $S_{2}$ with the corresponding component in $m_{1}$.

Theorem 6.33

Given $S_{1}$ sig, $S_{1} \vdash S_{2}$ sig, $m_{1} \in S_{1}$ and $m_{2} \in S_{2}\left(\mid m_{1}\right)_{S_{1}}$ then $m_{1} \oplus m_{2} \in S_{1} \oplus S_{2}$

Proof Similar to the proof of the type correctness of $\oplus$ given in Figure 6.5

The empty computational element is a right and left identity for $\oplus$ :

Theorem 6.34 $m \oplus$ module end $=m$

Proof Omitted $\square$

Theorem 6.35 module end $\oplus m=m \quad \square$

Proof Omitted $\square$

Theorems 6.36-6.39 are a collection of laws used to unfold applications of computational element concatenations to canonical computational elements. These laws can be used to express a canonical computational element as the concatenation of some smaller computational elements.

Theorem 6.36 module $y=e$ end $\oplus \operatorname{module} b$ end $=\operatorname{module} y=e, b$ end

Proof Given in Figure 6.6

Theorem 6.37 module $\bullet y=e$ end $\oplus \operatorname{module} b$ end $=\operatorname{module} \bullet y=e, b$ end $\square$

Proof Similar to that of Theorem 6.36 above $\square$

Theorem 6.38 module $y=e, b_{1}$ end $\oplus \operatorname{module} b_{2}$ end $=$ module $y=e$ end $\oplus \operatorname{module} b_{1}(y \backslash e)$ end $\oplus$ module $b_{2}$ end

Proof Similar to that of Theorem 6.36 above $\square$

Theorem 6.39 module $\bullet y=e, b_{1}$ end $\oplus$ module $b_{2}$ end $=$ module $\bullet y=e$ end $\oplus \operatorname{module} b_{1}(y \backslash e)$ end $\oplus$ module $b_{2}$ end

Proof Similar to that of Theorem 6.36 above

Theorem 6.40 (below) says that computational element concatenation is associative. The proof of Theorem 6.40 relies on the associativity of value tuple concatenation: 


\section{Proof (of Theorem 6.36)}

$\llbracket$ module $y=e$ end $\oplus$ module $b$ end $\rrbracket$

$=$ "definition of translation function over $\oplus$ (Fact 6.34)"

$\llbracket$ module $y=e$ end $\rrbracket \llbracket$ module $b$ end $\rrbracket$

$=$ "definition of translation mapping on computational elements, twice"

$\langle[\operatorname{inl}(\llbracket y \rrbracket)],\langle\llbracket e \rrbracket, t t\rangle\rangle \oplus\left\langle\llbracket\right.$ module $b$ end $\rrbracket_{\text {dom }}, \llbracket$ module $b$ end $\left.\rrbracket_{\text {val }}\right\rangle$

$=$ "definition of $\oplus$ "

$\left\langle[\operatorname{inl} l(\llbracket y \rrbracket)]+\llbracket\right.$ module $b$ end $\rrbracket_{\text {dom }},\langle\llbracket e \rrbracket, t t\rangle \odot \llbracket$ module $b$ end $\left.\rrbracket_{\text {val }}\right\rangle$

$=$ "definition of $\odot$ "

$\left\langle[\operatorname{inl}(\llbracket y \rrbracket)]+\llbracket\right.$ module $b$ end $\rrbracket_{d o m},\left\langle\llbracket e \rrbracket, \llbracket\right.$ module $b$ end $\left.\left.\rrbracket_{v a l}\right\rangle\right\rangle$

$=$ "definitions of $\llbracket-\rrbracket_{d o m}$ and $\llbracket-\rrbracket_{\text {val }}$ "

$\left\langle\llbracket\right.$ module $y=e, b$ end $\rrbracket_{\text {dom }}, \llbracket$ module $y=e, b$ end $\left.\rrbracket_{\text {val }}\right\rangle$

$=$ "definition of translation mapping on computational elements"

$\llbracket$ module $y=e, b$ end $\rrbracket$

Figure 6.6: A proof of Theorem 6.36

Lemma 6.6 Given $P$ lsg, $Q$ lsg and $R$ lsg; and $p \in P, q \in Q$ and $r \in R$, then $p \odot(q \odot r)=(p \odot q) \odot r \in(P \otimes Q \otimes R) \square$

\section{Proof Omitted $\square$}

\section{Theorem 6.40 (Associativity of $\oplus$ )}

Given $S_{1}$ sig, $S_{2}$ sig, $S_{3}$ sig, and $m_{1} \in S_{1}, m_{2} \in S_{2}, m_{3} \in S_{3}$ then:

$\left(m_{1} \oplus m_{2}\right) \oplus m_{3}=m_{1} \oplus\left(m_{2} \oplus m_{3}\right) \in\left(S_{1} \oplus S_{2}\right) \oplus S_{3}=S_{1} \oplus\left(S_{2} \oplus S_{3}\right)$

\section{Proof}

$$
\begin{aligned}
& \left(m_{1} \oplus m_{2}\right) \oplus m_{3} \\
= & \text { "definition of } \oplus " \\
& \left\langle\operatorname{dom}\left(m_{1} \oplus m_{2}\right)+\operatorname{dom}\left(m_{3}\right), \operatorname{val}\left(m_{1} \oplus m_{2}\right) \odot \operatorname{val}\left(m_{3}\right)\right\rangle \\
= & \text { "definition of } \oplus " \\
& \left\langle\left(\operatorname{dom}\left(m_{1}\right)+\operatorname{dom}\left(m_{2}\right)\right)+\operatorname{dom}\left(m_{3}\right),\left(\operatorname{val}\left(m_{1}\right) \odot \operatorname{val}\left(m_{2}\right)\right) \odot \operatorname{val}\left(m_{3}\right)\right\rangle \\
= & \text { "associativity of }+, \text { associativity of } \odot(\operatorname{Lemma} 6.6) " \\
& \left\langle\text { dom }\left(m_{1}\right)+\left(\operatorname{dom}\left(m_{2}\right)+\operatorname{dom}\left(m_{3}\right)\right), \operatorname{val}\left(m_{1}\right) \odot\left(\operatorname{val}\left(m_{2}\right) \odot \operatorname{val}\left(m_{3}\right)\right)\right\rangle \\
= & \text { "definition of } \oplus " \\
& \left\langle\text { dom }\left(m_{1}\right)+\operatorname{dom}\left(m_{2} \oplus m_{3}\right), \operatorname{val}\left(m_{1}\right) \odot \operatorname{val}\left(m_{2} \oplus m_{3}\right)\right\rangle \\
= & \text { ddefinition of } \oplus " \\
& m_{1} \oplus\left(m_{2} \oplus m_{3}\right)
\end{aligned}
$$




\subsubsection{Projection Functions on Computational Elements}

In this section, we describe two operators that split a computational element into two separate computational elements. We use these operators to factor large computational elements into smaller pieces. This is useful when we want to reason about some, but not all, of the components in a large computational element.

Given any two signatures $S_{1}$ and $S_{2}$, and any two computational elements $m_{1} \in S_{1}$ and $m_{2} \in S_{2}$, the projection function ${ }_{\Pi_{1}}^{S_{1} S_{2}}$ takes $m_{1} \oplus m_{2} \in S_{1} \oplus S_{2}$ and returns $m_{1} \in S_{1}$. The projection function $\frac{S_{1} S_{2}}{\Pi_{2}}$ takes $m_{1} \oplus m_{2} \in S_{1} \oplus S_{2}$ and returns $m_{2} \in S_{2}$. The signature $S_{2}$ may be dependent on $S_{1}$. In that case, $\underset{\Pi_{2}}{s_{1} S_{2}}\left(m_{1} \oplus m_{2}\right)=m_{2} \in S_{2}\left(\left|m_{1}\right| S_{1}\right.$.

Remark We omit the formal definitions of ${ }_{\Pi_{1}}^{s_{1} s_{2}}$ and ${ }_{\Pi_{1} s_{2}}$, but it may help the reader to know that we define ${ }_{\Pi_{1}}^{s_{1} s_{2}}$ and ${ }_{\Pi_{2}}^{s_{1} s_{2}}$ using dot notation on computational elements $\square$

We will only require the properties stated in Facts 6.23-6.25, below; in each of these facts we assume that $S_{1}$ sig, $S_{1} \vdash S_{2}$ sig, $m_{1} \in S_{1}, m_{2} \in S_{2}\left(\left|m_{1}\right| S_{1}\right.$ and $m \in S_{1} \oplus S_{2}$.

Fact $6.23 \stackrel{s_{1} s_{2}}{\Pi_{1}}\left(m_{1} \oplus m_{2}\right)=m_{1} \in S_{1} \square$

Fact $6.24 \underset{\Pi_{2}}{s_{1} S_{2}}\left(m_{1} \oplus m_{2}\right)=m_{2} \in S_{2}\left(\mid m_{1}\right)_{S_{1}}$

Fact $6.25 \quad \begin{gathered}s_{1} S_{2} \\ \Pi_{1}\end{gathered}(m) \oplus \underset{\Pi_{2}}{s_{1} S_{2}}(m)=m \in\left(S_{1} \oplus S_{2}\right) \square$

\subsection{Translating Computational Element Opera- tors}

In this section, we summarise the syntax of the computational element operations defined in this chapter, and we give some useful facts about the operations. The grammar ceop, in Definition 6.22, gives the syntax of computational element operators; token $m$ (possibly subscripted) stands for ceop terms. Note that ceop includes comp which is the grammar for canonical computational elements. We assume that ceop is added to $\operatorname{Exp}$ (i.e. Exp $::=$ ceop).

\section{Definition 6.22 (Syntax of Computational element Operators)}

$$
\text { сеор ::= comp }|m[p \backslash q]| m \backslash i \mid m_{1} \oplus m_{2}
$$

We obtain the following facts from the definition of the translation mapping on canonical computational elements, which is $\llbracket m \rrbracket=\left\langle\llbracket m \rrbracket_{\text {dom }}, \llbracket m \rrbracket_{\text {val }}\right\rangle$ for any $m$ : comp; recall that $\llbracket-\rrbracket_{d o m}$ and $\llbracket-\rrbracket_{v a l}$ translate canonical computational elements into their domains and value-tuples, respectively.

Fact $6.26 \llbracket m[p \backslash q] \rrbracket_{d o m}=\llbracket m \rrbracket_{d o m}\left[\llbracket p \rrbracket_{I d} \rrbracket \llbracket q \rrbracket_{I d}\right] \square$ 


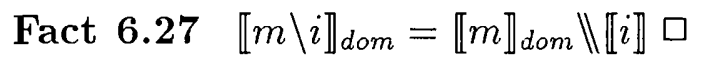

Fact $6.28 \llbracket m_{1} \oplus m_{2} \rrbracket_{d o m}=\llbracket m_{1} \rrbracket_{d o m}+\llbracket m_{2} \rrbracket_{d o m} \square$

Fact $6.29 \llbracket m[p \backslash q] \rrbracket_{v a l}=\llbracket m \rrbracket_{v a l} \square$

Fact 6.30 $\llbracket m \backslash i \rrbracket_{v a l}=\llbracket m \rrbracket_{v a l} \square$

Fact 6.31 $\llbracket m_{1} \oplus m_{2} \rrbracket_{v a l}=\llbracket m_{1} \rrbracket_{v a l} \odot \llbracket m_{2} \rrbracket_{v a l} \square$

Fact 6.32 $\llbracket m[p \backslash q] \rrbracket=\llbracket m \rrbracket\left[\llbracket p \rrbracket_{I d} \backslash \llbracket q \rrbracket_{I d}\right\rfloor \square$

Fact 6.33 $\llbracket m \backslash i \rrbracket=\llbracket m \rrbracket \backslash i \rrbracket \square$

Fact 6.34 $\llbracket m_{1} \oplus m_{2} \rrbracket=\llbracket m_{1} \rrbracket+\llbracket m_{2} \rrbracket \square$

\subsection{Inverse Operators}

In this section, we define the inverses of the computational element renaming and hiding operators. These inverse operators will be needed, in Chapter 7, to define specification operators that rename and hide components in specifications. The inverse operators will also be used in Chapter 8 to define some refinement laws.

\subsubsection{Inverse of Computational Element Renaming}

Given any signature $S$ and component names $p, q \in \mathbb{S}$, then the inverse of the computational element renaming operator $-[p \backslash q] \in S \rightarrow S[p \backslash q]$ is written as $-[p \backslash q]_{S}^{-1}$ and has the type $S[p \backslash q] \rightarrow S$. Note that ${ }_{-}[p \backslash q]_{S}^{-1}$ is subscripted by its range $S$. The following facts say that ${ }_{-}[p \backslash q]_{S}^{-1}$ is the inverse of $-[p \backslash q]$ :

Fact 6.35 $(m[p \backslash q])[p \backslash q]_{S}^{-1}=m \in S$ where $S$ sig and $m \in S \square$

Fact 6.36 $\left(m[p \backslash q]_{S}^{-1}\right)[p \backslash q]=m \in S[p \backslash q]$ where $S$ sig and $m \in S[p \backslash q] \square$

We give a formal definition of ${ }_{-}[p \backslash q]_{S}^{-1}$ in Definition 6.23. We justify Definition 6.23 by proving that $(m[p \backslash q])[p \backslash q]_{S}^{-1}=m$ (i.e. Fact 6.35 ), and the proof follows Definition 6.23:

\section{Definition 6.23}

Given any $S$ sig, names $p, q \in \mathbb{S}$ and $m \in S[p \backslash q]$ then $-[p \backslash q]_{S}^{-1} \in S[p \backslash q] \rightarrow S$ is defined as follows:

$$
m[p \backslash q]_{S}^{-1}=\langle\operatorname{Dom}(S), \operatorname{val}(m)\rangle \in S
$$




\section{Proof (of Fact 6.35)}

Consider any $m \in S$ where $S$ is any signature. By Definition 4.8, the domains of $m$ and $S$ are identical, i.e. $\operatorname{dom}(m)=\operatorname{Dom}(S) \in \mathbb{D}$. By Definition 6.16, the value tuple of $m[p \backslash q]$ is identical to the value tuple of $m$, i.e. $\operatorname{val}(m)=\operatorname{val}(m[p \backslash q]) \in \operatorname{Lsg}(S)$. The proof of Fact 6.35 is:

$$
\begin{aligned}
&(m[p \backslash q])[p \backslash q]_{S}^{-1} \in S \\
&= \text { "Definition } 6.23 " \\
&\langle\operatorname{Dom}(S), \operatorname{val}(m[p \backslash q])\rangle \in S \text { "} \operatorname{dom}(m)=\operatorname{Dom}(S), \operatorname{val}(m)=\operatorname{val}(m[p \backslash q]) " \\
&\langle\operatorname{dom}(m), \operatorname{val}(m)\rangle \in S \\
&= \text { "Fact } 4.5 " \\
& m \in S
\end{aligned}
$$

\subsubsection{Inverse of Computational Element Hiding}

Given any signature $S$ and set of names $i \in \operatorname{Set}(\mathbb{S})$, then the inverse of $-\backslash i \in S \rightarrow S \backslash i$ is written as $-\backslash S_{S}^{-1} i$ and has the type $S \backslash i \rightarrow S$. Note that $-\backslash_{S}^{-1} i$ is subscripted by its range $S$. The following facts say that $-\backslash S^{-1} i$ is the inverse of $-\backslash i$ :

Fact $6.37(m \backslash i) \backslash S^{-1} i=m \in S$ where $S$ sig and $m \in S \square$

Fact $6.38\left(m \backslash{ }_{S}^{-1} i\right) \backslash i=m \in S \backslash i$ where $S$ sig and $m \in S \backslash i$

Definition 6.24 contains a formal definition of $-\backslash S_{S}^{-1} i$. A justification of the correctness of Definition 6.24 is similar to the justification of the inverse computational element renaming operator (Definition 6.23).

\section{Definition 6.24}

Given any $S$ sig, a set of names $i \in S e t(\mathbb{S})$, and $m \in S \backslash i$ then ${ }_{-} \backslash{ }_{S}^{-1} i \in S \backslash i \rightarrow S$ is defined as follows:

$$
m \backslash{ }_{S}^{-1} i=\langle\operatorname{Dom}(S), \operatorname{val}(m)\rangle \in S
$$

We finish this section by giving two properties of $-\backslash_{S}^{-1} i$. The first property is that the inverse operation to hiding an empty set of components is an identity operation:

Theorem 6.41 $m \backslash_{S}^{-1}\{\}=m$ where $S$ sig and $m \in S=S \backslash\{\}$

\section{Proof Omitted $\square$}

The second property is that consecutive applications of $-\backslash_{S}^{-1} i$ can be expressed as a single application of $-\backslash_{S}^{-1} i$ : 


\section{Theorem 6.42}

$\left(m \backslash \backslash_{S \backslash i}^{-1} j\right) \backslash{ }_{S}^{-1} i=m \backslash{ }_{S}^{-1}(i \cup j) \in S$ where $S$ sig, $i, j \in \operatorname{Set}(\mathbb{S})$ and $m \in S \backslash i \backslash j$

$$
\begin{gathered}
\text { Proof } \\
(m \backslash S \backslash i) \backslash S_{S}^{-1} i \in S \\
=\text { "definition of } m \backslash_{S \backslash i}^{-1} j " \\
\langle\operatorname{Dom}(S \backslash i), \operatorname{val}(m)\rangle \backslash_{S}^{-1} i \in S \\
=\text { "definition of } m \backslash S \backslash i \\
\langle\text { Dom }(S), \operatorname{val}(m)\rangle \in S \\
=\text { "definition of } m \backslash S \backslash(i \cup j) \\
m \backslash_{S}^{-1}(i \cup j) \in S
\end{gathered}
$$

\subsection{Discussion and Conclusion}

In the course of developing the operators given in this chapter, we have considered several alternative definitions for some of our operators. In particular, we have considered several solutions to resolving the name-clashes that arise when concatenating signatures. The most promising alternative solution involves merging components that have the same name. For example, consider the following signatures:

$$
S_{1}=y_{1} \in \mathbb{N}, y_{2} \in \mathbb{B} \text { and } S_{2}=y_{3} \in \mathbb{N} \times \mathbb{N}, y_{2} \in \mathbb{B}
$$

A signature combinator that merges common names might combines $S_{1}$ and $S_{2}$ to produce the following signature:

$$
S_{3}=y_{1} \in \mathbb{N}, y_{2} \in \mathbb{B}, y_{3} \in \mathbb{N} \times \mathbb{N}
$$

Note that the components named $y_{2}$ from $S_{1}$ and $S_{2}$ have been merged to a single component in $S_{3}$. In contrast, the signature concatenation of $S_{1}$ and $S_{2}$ contains two distinct components named $y_{2}$ :

$$
S_{1} \oplus S_{2}=y_{1} \in \mathbb{N}, y_{2} \in \mathbb{B}, y_{3} \in \mathbb{N} \times \mathbb{N}, y_{2} \in \mathbb{B}
$$

Many specification languages, such as ASL and Z, resolve name-clashes by merging components in the style illustrated above. The advantage of merging is that components with the same name are automatically identified as being equal. So, $y_{2}$ values from $S_{3}$ can be used in place of $y_{2}$ values from $S_{1}$ and $S_{2}$. However, there are problems with merging: if the types of two components with the same name are not equal, then they cannot always be merged. For example, if the type of $y_{2}$, in $S_{2}$, is changed to $\mathbb{N}$ then $y_{2}$ cannot be given a type in $S_{3}$, as we cannot merge $\mathbb{N}$ and $\mathbb{B}$. In $\mathrm{Z}$, components are only merged if they have the same name and type, and an attempt to compose two schemas in which the name-clashes cannot be resolved by merging is ill-defined. 
In contrast, our signature concatenation operation is a total operation which can combine any two signatures.

In order to merge components that have differing types, we have considered introducing an intersection type (*) that combines any two types $P$ and $Q$ to produce a type $P * Q$ containing the elements common to both $P$ and $Q$. However, even with such an intersection type, the dependencies within a signature may still prevent components being merged. For example, consider the following signatures:

$$
S_{4}=y_{1} \in A_{1}, y_{2} \in A_{2}\left(y_{1}\right) \text { and } S_{5}=y_{2} \in B_{2}, y_{1} \in B_{1}\left(y_{2}\right)
$$

Note that in $S_{4}$, the type of $y_{2}$ is dependent on $y_{1}$; and in $S_{5}$, the type of $y_{1}$ is dependent on $y_{2}$. If we use $(*)$ to merge the $y_{1}$ components in $S_{4}$ and $S_{5}$ then we get $y_{1} \in A_{1} * B_{1}\left(y_{2}\right)$, so that $y_{1}$ is dependent on $y_{2}$. Similarly, merging both $y_{2}$ components gives us $y_{2} \in B_{2} * A_{2}\left(y_{1}\right)$ which is dependent on $y_{1}$. However, there is no way of ordering the new definitions of $y_{1}$ and $y_{2}$ so that $y_{1}$ is dependent on $y_{2}$, and $y_{2}$ is dependent on $y_{1}$. Therefore, even with an intersection type, it is not always possible to merge the types of two components in a signature.

We have shown that there is an interesting relationship between each signature operator, and the corresponding operator on computational elements: given a computational element $m \in S$, if $f$ is a computational element operator, and $F$ is the corresponding signature operator, then $f(m) \in F(S)$. In Chapter 9, we will show that this relationship allows specifications that are made using the signature operators to be implemented using the corresponding computational element operators; and this property will allow the piecewise implementation of structured specifications.

One disadvantage of using MLT to define signature and computational element operators is that the semantics of these operators is complicated. One reason for this complexity is the very simplicity of MLT, and in particular, its lack of pre-defined operations. Consequently, we have defined many auxiliary operators-such as tuple and loose signature concatenation - in order to define our signature and computational element operators. The need to define so many auxiliary operators is irritating, but as a library of such operators is developed, so the need to define more auxiliary operations will be reduced. The complexity of the semantics is exacerbated by the need to define operations twice-once for signatures, and once for computational elements. This is a consequence of specifications and modules having different semantics in MLT.

It is possible to describe the signature and computational element operators at a purely syntactic level. Such a description has the same complexity as the one given in this chapter, and still requires us to define the auxiliary operators given in this chapter so that we can reason about our signature and computational element operators.

In summary, we have presented the definition of several signature and computational element operators. These operators will be used to define specification and module 
structuring operators. We have also supplied laws concerning the basic properties of each operator, and these laws will be used to justify laws about specification and module operators. 


\section{Chapter 7}

\section{Structured Specifications}

\subsection{Introduction}

Large specifications are as complex in their structure as programs. It is convenient to construct them, methodically, in small pieces which we then combine to make larger specifications. Such a style supports the modular decomposition of specifications into manageable pieces, and also helps to control the complexity arising from a large number of type, value and function symbols. Structuring a specification also helps to isolate its individual parts from changes to other parts of the specification; this minimises the amount of re-specification required when we change part of a specification. It is also more likely that we can reuse the individual parts of a structured specification, and this encourages the building of libraries of reusable specifications. In order to support the construction of structured specifications, a specification language requires structuring operators which can both modify and combine specifications to make new specifications. Our purpose in this chapter is to define some structuring operations on specifications, and consider their role in making structured specifications.

Our choice of specification operators is determined by how we intend to make specifications. We take the view that, where possible, specifications should be constructed in an incremental manner where we continually add detail to a specification until we are satisfied it specifies what we want. For example, we often wish to add new components and restrictions to an existing specification; so, we will require some form of enrichment operator. We may wish to make a specification by combining several independently developed specifications, so we will require some form of sum operator to combine the components of two specifications. We may also wish to reuse existing specifications to make new specifications; so, we will require operators to "customise" existing specifications to their new role by renaming, or hiding, some of their components, for example. 
Several specification languages, such as CLEAR [5], ASL [51] and Z [52], supply specification operators that support the construction of structured specifications; readers familiar with such languages should find some of our specification operators familiar. However, unlike the specification languages mentioned above, our specification operators are defined in a type-theoretic framework. This approach confers certain benefits when implementing and deducing properties from structured specifications. For example, we can use the constructive logic available in type theory to prove properties of the operators. In Chapter 9, we will show that for some specification operators there exist corresponding module operators that we can use to implement structured specifications; this fact is a consequence of the fact that specifications are types, and their implementations are members of these types.

We will give some basic laws of the specification operators. These laws can be used to deduce the behaviour of a software system from its specification: we can formulate some required properties of a system and then use the laws to prove that its specification implies these properties. The laws can also be used to change the structure of a specification prior to, or during, its refinement and implementation: we may have to rearrange a specification if it has a structure that, if carried over to an implementation, yields an unacceptably inefficient implementation.

The formal definitions of some of the specification operators are more complicated than we might expect. We will show that the complexity of these definitions is necessary to ensure that the operators are well-typed.

This chapter is organised as follows. We begin by introducing our specification operators, and then give an example that shows how they can be used to incrementally construct specifications. Next, we give the formal semantics of each operator. Finally, we state and prove some basic properties of the specification operators.

\subsection{The Specification Operators}

In this section, we introduce a small collection of specification operators. Figure 7.1 contains a syntax summary of the specification operators. Some of the operators, such as rename, hide and sum, should be familiar to reader acquainted with algebraic specification languages, or $\mathrm{Z}$, while other operators, such as derive and translate, may be less familiar. Note that we overload the signature hiding and renaming notation and use it to denote the rename and hide operators, respectively. We will give an informal description of the properties of each specification operator and consider how they address some of the issues discussed in the introduction. The operators defined here are not the only operators possible; however, we believe them to be a useful collection and they are sufficient for our purposes. 


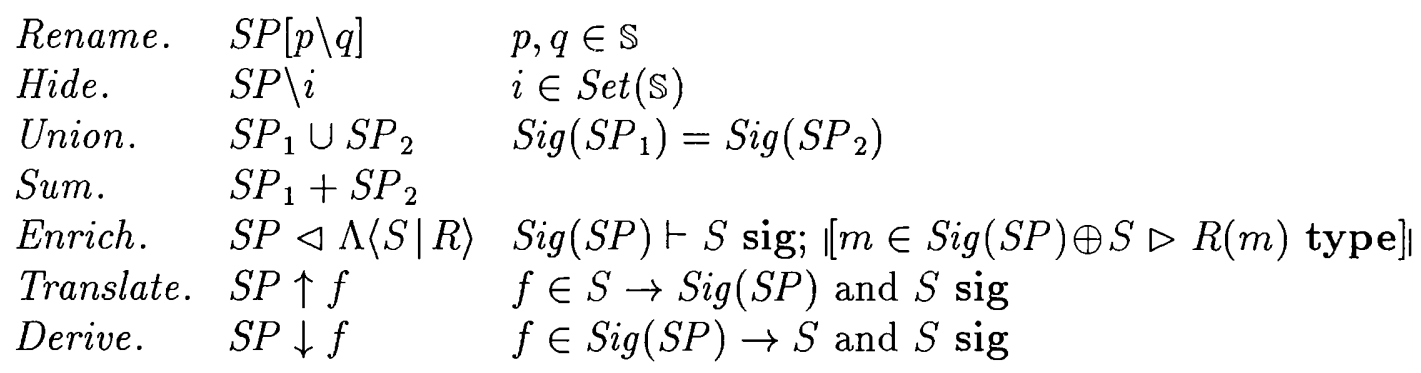

Figure 7.1: The Specification Operators

In what follows, it may be helpful to think of specification operators as functions that take a number of arguments, including specifications, and return specifications as result. Examples of using the operators are left until Section 7.3; and the formal semantics of the specification operators are left until Section 7.4.

Renaming The rename operator allows a specifier to rename the components supplied by the signature of a specification. Renaming a component $p$ to $q$ in specification $S P$ is written as $S P[p \backslash q]$. Renaming is a surprisingly useful operation. For example, renaming allows us to reuse an existing specification whose component names are not meaningful in the context in which it is reused. Renaming can also be used to prevent name-clashes when combining specifications-we shall see examples of this latter. Renaming is defined using signature renaming, and has similar properties to signature renaming.

Hiding The hide operator allows a specifier to modify an existing specification by making visible components local to the signature of a specification. An application of hide has the form $S P \backslash i$ for any specification $S P$ and $i$ a set of component names. $S P \backslash i$ is $S P$ with all visible components named in $i$ made local in $S P$. Intuitively, $S P \backslash i$ puts a bullet $(\bullet)$ in front of the declaration of any visible component of $S P$ named in $i$. hide is defined using signature hiding.

Union The union operator merges two specifications, provided they have the same signature. The union of two specifications $S P_{1}$ and $S P_{2}$ is written as $S P_{1} \cup S P_{2}$, assuming $\operatorname{Sig}\left(S P_{1}\right)=\operatorname{Sig}\left(S P_{2}\right)$. The specification $S P_{1} \cup S P_{2}$ has the same signature as $S P_{1}$ and $S P_{2}$, but its restriction is the conjunction of the restrictions of $S P_{1}$ and $S P_{2}$. Consequently, the implementations of $S P_{1} \cup S P_{2}$ are exactly those modules that satisfy both $S P_{1}$ and $S P_{2}$.

Sum The sum of two arbitrary specifications $S P_{1}$ and $S P_{2}$ is written as $S P_{1}+S P_{2}$. The sum operator allows us to make individual specifications in isolation and then 
combine them to make a new specification. $S P_{1}+S P_{2}$ supplies all the components supplied by $S P_{1}$ and $S P_{2}$ : the signature of $S P_{1}+S P_{2}$ is the signature concatenation of $\operatorname{Sig}\left(S P_{1}\right)$ and $\operatorname{Sig}\left(S P_{2}\right)$. The restriction of $S P_{1}+S P_{2}$ is the conjunction of the restrictions of $S P_{1}$ and $S P_{2}$. Note that sum differs from union as it allows us to combine specifications with differing signatures. One of the issues that arises when defining sum is resolving the name-clashes that can occur if some component names in $S P_{1}$ are also used as component names in $S P_{2}$. sum resolves name-clashes in the same way as signature concatenation, by overloading any component name that appears in both its arguments. For example, if $S P_{1}$ and $S P_{2}$ both specify a component named $y$, then $S P_{1}+S P_{2}$ specifies both those components; in other words, $S P_{1}+S P_{2}$ supplies two, distinct, components named $y$.

Enrichment The enrich operator allows us to modify a specification by adding new components and restrictions. Enriching a specification $S P$ with the components supplied by a signature $S$, and adding the restriction $R$, is written as $S P \triangleleft \Lambda\langle S \mid R\rangle$. The expression $\Lambda\langle S \mid R\rangle$ is called an enrichment. $S$ is dependent on $\operatorname{Sig}(S P)$ (i.e. $\operatorname{Sig}(S P) \vdash S$ sig holds), so the components supplied by $S$ may be dependent on the components of $S P$. The signature of $S P \triangleleft \Lambda\langle S \mid R\rangle$ is the signature concatenation of $\operatorname{Sig}(S P)$ and $S$ (i.e. $\operatorname{Sig}(S P) \oplus S)$ ). $R$ is dependent on any $m \in \operatorname{Sig}(S P) \oplus S$, so $R$ may be defined using any components from $S P$ and $S . \Lambda\langle S \mid R\rangle$ is not a specification since $R$ depends on computational elements that meet $\operatorname{Sig}(S P) \oplus S$, instead of those that meet $S$; this is the main difference between sum and enrich. We will sometimes write enrichements of the form $\Lambda\langle S \mid R\rangle$ as $\Lambda$ Elements $S$ Restrictions $R$ End.

Derive An application of derive has the form $S P \downarrow f$ for any $f \in \operatorname{Sig}(S P) \rightarrow S$ and $S$ any signature. Functions such as $f$, that are used by derive are called derive maps. If we consider $S P \downarrow f$ as a type, its members are all $m \in S P$, but with $f$ applied to the computational element of each $m$ (i.e. $S P \downarrow f$ specifies any module whose computational element is in the set $\{f(c e(m)) \mid m \in S P\})$. For example, we may use the computational element renaming operator $-[p \backslash q] \in \operatorname{Sig}(S P) \rightarrow \operatorname{Sig}(S P)[p \backslash q]$ as a derive map, to produce the specification $S P \downarrow_{(-[p \backslash q])}$ that is equivalent to $S P[p \backslash q]$. derive is a versatile operator: with appropriate choices of derive map, derive can be used to permute, rename, and remove components from the signature of a specification. derive can also be used to change the type of components in the signature of a specification. The only conditions on $f$ are that its domain is $\operatorname{Sig}(S P)$ and its range is a signature; the range of $f$ is the signature of $S P \downarrow f$. In Section 7.3, we will describe how derive can be used to remove components from a specification. For readers familiar with algebraic specifications, derive is a generalisation of the Derive operation in the specification language ASL [51]. 
Translate An application of translate has the form $S P \uparrow f$ for any $f \in S \rightarrow$ $\operatorname{Sig}(S P)$ and $S$ any signature. Functions such as $f$, that are used by translate are called translate maps. $S P \uparrow f$ specifies any module whose computational element meets $S$ and maps, under $f$, to a computational element that satisfies the restriction of $S P$ (i.e. $S P \uparrow f$ specifies any module whose computational element is in the set $\{m \in S \mid A x(S P)(f(m))\})$. For example, if $f$ is the inverse computational element renaming operator $-[p \backslash q]_{\text {Sig }(S P)}^{-1} \in \operatorname{Sig}(S P)[p \backslash q] \rightarrow \operatorname{Sig}(S P)$ then $S P \uparrow f$ is equivalent to $S P[p \backslash q]$. With appropriate choices of translate map, translate can be used to permute, rename, or insert components into a specification. The reader might be forgiven for thinking that derive and translate are so similar that we can do without one. However, there are many transformations that can be defined by derive, but not translate, and vice-versa: we illustrate this in an example, later. For those readers familiar with algebraic specifications, translate is a generalisation of the operation Translate defined in [50].

\subsection{An Example Structured Specification}

In this section, we give an example of constructing a structured specification. As our example, we specify some modules that might be used as part of a book library system. We intend to use the specifications Catalogue and BookSpec given previously in Figures 1.2 and 1.4, respectively. Recall that Catalogue supplies a type named Stock which is used to represent collections of books; Catalogue also supplies operations to add books to Stock values; remove books from Stock values; and also includes operations to query the availability of a book. BookSpec supplies a type named Book, together with operations on Book values; for example, there is an operation to create new book values, as well as operations to query the title and author of a book. We show how the structuring operators can be used to construct a new specification of a book catalogue in an incremental style. We also show how the specification operators facilitate the reuse of Catalogue and BookSpec to specify a module for a register of library users. Our aim is only to illustrate the use of the specification operators and we do not present a complete specification of a library system.

\subsubsection{A Book Catalogue}

We begin by considering how to combine Catalogue and BookSpec to produce a new catalogue specification supplying the components in Catalogue and BookSpec. Inspection of Catalogue and BookSpec reveals that they both supply a type named Book. When we combine Catalogue and BookSpec, we would like to ensure that the values of the type Book specified by Catalogue are compatible with the values of Book specified 
by BookSpec. Let us consider trying to combine Catalogue and BookSpec using the sum operator. If we sum Catalogue and BookSpec directly, the resultant specification will contain all the components of Catalogue and BookSpec, including two versions of the type Book (i.e. Book is overloaded). We avoid overloading Book-for reasons that will be made clear below-by renaming the component Book in BookSpec to Book $_{2}$ prior to summing Catalogue and BookSpec:

$$
\text { Catalogue }_{2}=\text { Catalogue }+ \text { BookSpec }\left[\text { Book } \backslash \text { Book }_{2}\right]
$$

Catalogue $_{2}$ does not quite specify the catalogue module we desire, as it does not specify that values of type $B o o k$ and $B o o k_{2}$ are compatible: compatibility means that $B o o k$ values can be used in place of $B o o k_{2}$ values, and vice versa. Therefore, we use the enrich operator to add the constraint that Book and Book $k_{2}$ should be equal:

$$
\text { Catalogue }_{3}=\text { Catalogue }_{2} \triangleleft \Lambda\left\langle\Phi \mid B o o k={ }_{U_{1}} B o o k_{2}\right\rangle
$$

Note that the signature of the enrichment is $\Phi$ as we do not wish to add new components to Catalogue $_{2}$. If we had not renamed Book to Book ${ }_{2}$ then we would be unable to specify the enrichment above. This is because Book is overloaded in Catalogue + BookSpec and, hence, the version of Book from Catalogue is not in scope in the restriction of Catalogue + BookSpec, or any enrichment of the restriction (the scope rules for overloaded names establish that Book, from BookSpec, is in scope, rather than Book from Catalogue).

Since $B_{o o k}$ is equivalent to $B o o k$, we choose to hide $B o o k_{2}$ so that users of Catalogue $_{3}$ can only use $B o o k$ as the type of books; $B o o k_{2}$ is relegated to an internal role.

$$
\text { Catalogue }_{4}=\text { Catalogue }_{3} \backslash\left\{\text { Book }_{2}\right\}
$$

$\mathrm{Book}_{2}$ is a local component in Catalogue 4 , but this does not cause any problems with the use of Catalogue $_{4}$. Book 2 can be removed entirely, and the removal of components is discussed in Section 7.3.3.

Catalogue $_{4}$ concludes the specification of the book catalogue. Using the specification operators, we have been able to construct the book catalogue in an incremental

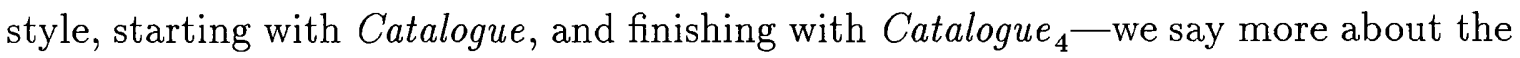
incremental construction of specifications in Section 7.3.4.

Although Catalogue $_{4}$ is a structured specification, it can still be "flattened" into a canonical specification. In practice, the structure of a specification should always be maintained, as it makes the specification easier to understand. However, to help confirm the readers intuition about the specification operators used in Catalogue $_{4}$, we unfold Catalogue $_{4}$ to an equivalent-under specification equality $(\Leftrightarrow)$-canonical specification named Catalogue $_{5}$ (i.e. Catalogue C $_{4} \Leftrightarrow$ Catalogue $_{5}$ ). Catalogue Ca $_{5}$ iven in Figure 7.2. We omit the details of producing Catalogue ${ }_{5}$, but the reader should be aware that work needs to be done to unfold Catalogue $_{4}$ to Catalogue $_{5}$-we unfold Catalogue $_{4}$ to Catalogue ${ }_{5}$ using theorems given later, in Section 7.5. 


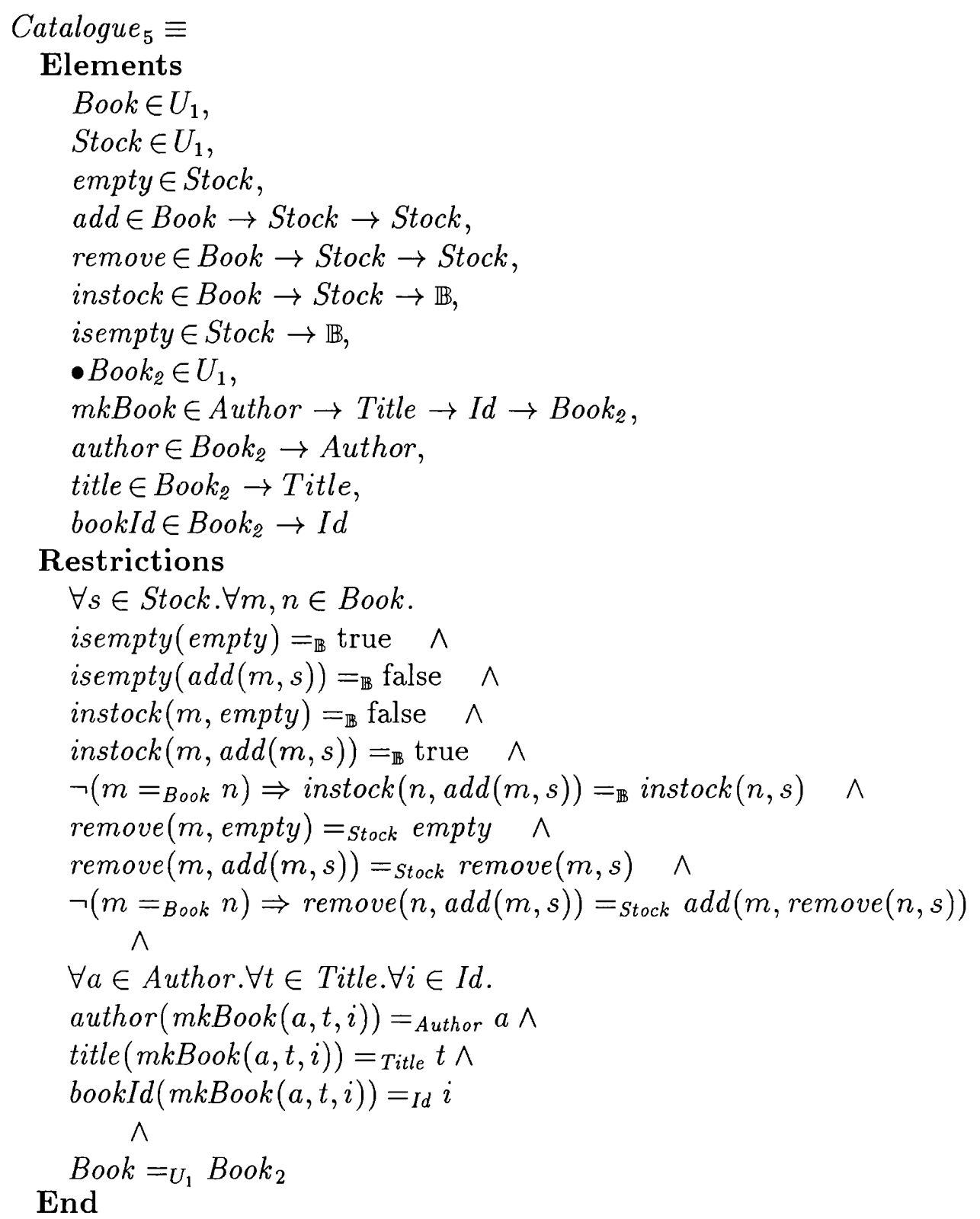

Figure 7.2: Expansion of Catalogue $_{4}$ 


\subsubsection{A Register of Library Users}

If we continue to develop a library specification, then we might wish to specify a module for a register of library users. Such a specification would be very similar to Catalogue, as we would require operations to add and remove library users to and from a register. We would also require operations to query the status of a register and library users. One possible specification of a register module may be defined by simply renaming some of the components of Catalogue, giving the following specification:

$$
\begin{aligned}
& \text { Register }=\text { Catalogue }[\text { Book } \backslash \text { Person, Stock } \backslash \text { Users, add } \backslash \text { addUsers, } \\
& \text { remove } \backslash \text { RemoveUsers, instock } \backslash \text { registered ] }
\end{aligned}
$$

In practice, when we reuse a specification, such as Catalogue, it may contain components that we don't require, and these may be hidden using hide, or derive. Additionally, we may want to add some new components defined in terms of existing components, and these may be added using the enrich operator. For example, suppose we require an extra operation within a register module, so that we can calculate the number of registered users. Such an operation may be specified as an enrichment of Register:

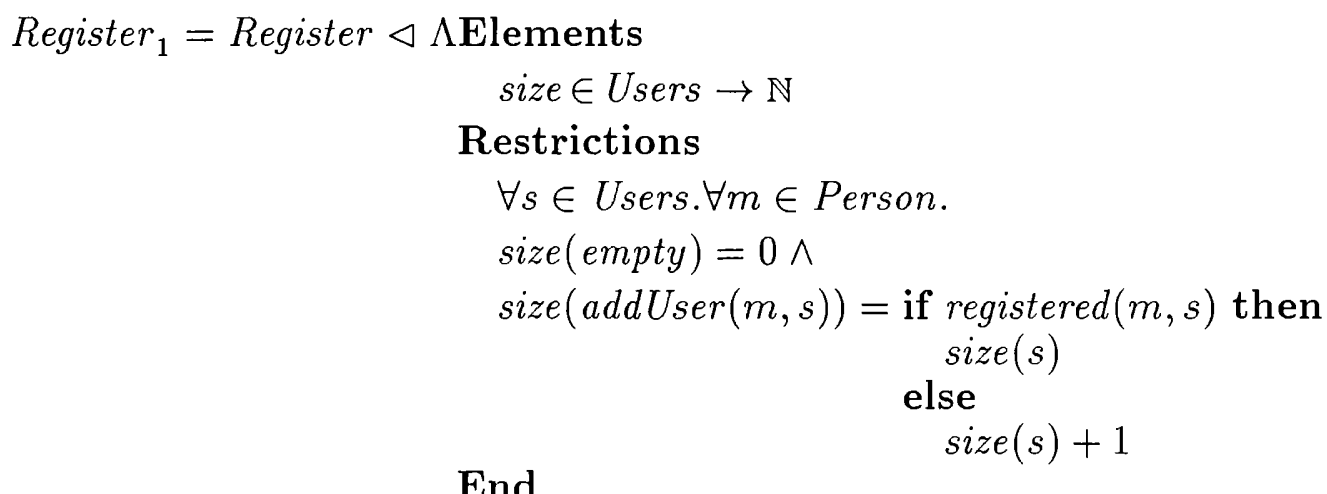

In any register, we might keep the name, address and personal identifier of each library user. So we reuse Bookspec to specify a module containing a type, named Person, that is used to represent library users:

$$
\begin{aligned}
\text { PersonSpec }=\text { BookSpec }\left[\begin{array}{l}
\text { Book } \backslash \text { Person }, m k B o o k \backslash m k P e r s o n, \\
\text { author } \backslash \text { name, title } \backslash \text { address, bookId } \backslash \text { personId }]
\end{array}\right.
\end{aligned}
$$

We now aim to produce a specification that specifies all the components supplied by Register and PersonSpec, such that the type Person in Register is compatible with the type Person in PersonSpec, and vice versa. There are several ways of combining Register and PersonSpec to specify a register of library users, and in the following we consider two possible solutions. 




Figure 7.3: A Signature $S$

The task of combining Register and PersonSpec is similar to that of combining Catalogue and BookSpec. So, one solution is to use the same style of combination used to construct Catalogue $_{3}$ :

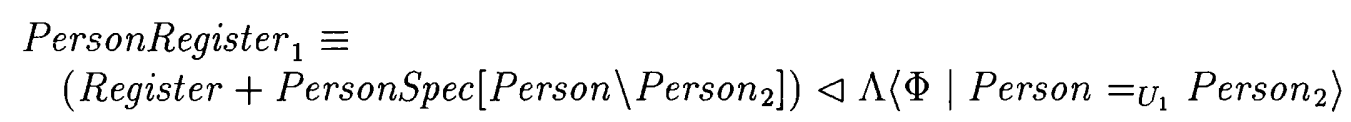

Our main criticism of PersonRegister ${ }_{1}$ is that Person $_{2}$ is redundant since it is equivalent to Person. If we use this style of combination too often then we can end up with specifications that are "cluttered" with many redundant components.

It is possible to combine Register and PersonSpec to produce a specification in which Person $_{2}$ and Person are merged into a single component, and we now describe how to make such a specification. We will use translate to transform Register and PersonSpec into specifications with a common signature and then merge those specifications using the union operator. The common signature is called $S$, and is given in Figure 7.3. $\llbracket S \rrbracket$ sig holds, and we use $S$ as a type (i.e. as $\llbracket S \rrbracket)$. $S$ contains all the components of Register and PersonSpec, but Person only appears once.

In order to use translate to transform the signatures of Register and PersonSpec to $S$, we require the functions

$$
\begin{aligned}
& f \in S \rightarrow \text { Sig(Register }) \text { and } \\
& g \in S \rightarrow \text { Sig(PersonSpec })
\end{aligned}
$$

given in Figures 7.4 and 7.5, respectively. Function $f$ takes any computational element $m \in S$ and removes all the components of $m$ that are not specified by Register; the result is a computational element of type Sig(Register). Similarly, $g$ throws away any components not specified by PersonSpec, to produce a computational element of type Sig(PersonSpec). We use $f$ and $g$ to make the following specifications:

$$
\begin{array}{ll}
\text { Register }_{2} & =\text { Register } \uparrow f \\
\text { PersonSpec }_{2} & =\text { PersonSpec } \uparrow g
\end{array}
$$




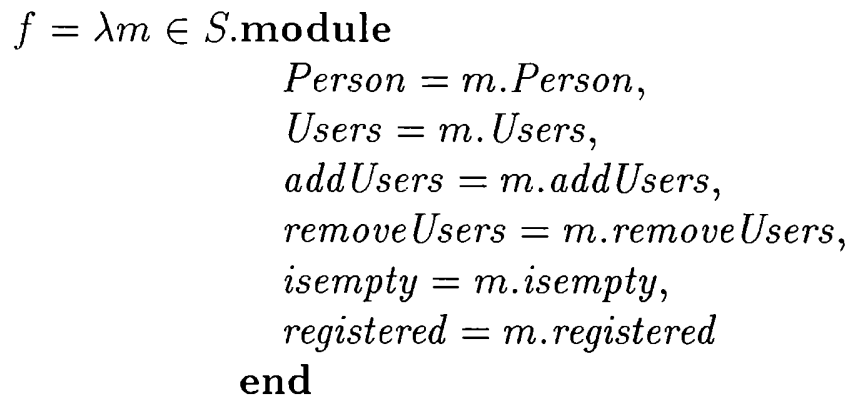

Figure 7.4: A function $f \in S \rightarrow \operatorname{Sig}($ Register $)$

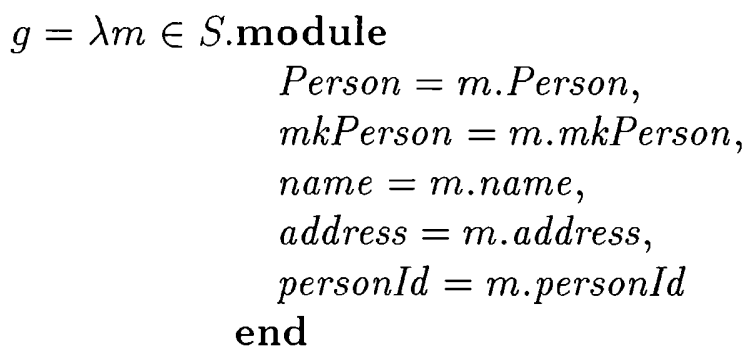

Figure 7.5: A function $g \in S \rightarrow \operatorname{Sig}($ PersonSpec)

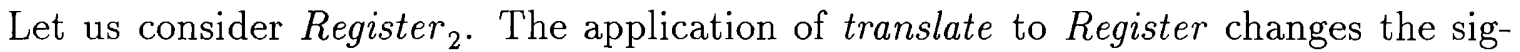
nature of Register to $S$ by adding extra components mkPerson, name, address and personId. The restrictions of Register $_{2}$ and Register are equivalent. Consequently, the components of Register ${ }_{2}$ that are common to Register have the same behaviour as the components of Register. The components of Register ${ }_{2}$ which are common to PersonSpec are left unconstrained. A more technical description of Register ${ }_{2}$ is that it is a type containing modules whose computational elements are in the set $\{m \in S \mid$ Ax (Register $)(f(m))\} ; f$ "translates" each $m \in S$ to $f(m) \in$ Register in order to verify that the components of $m$ that are specified by Register satisfy the restriction of Register. PersonSpec ${ }_{2}$ is defined in a similar style to Register ${ }_{2}$. PersonSpec $_{2}$ has signature $S$, and has the same restriction as PersonSpec.

Finally, we take the union of Register ${ }_{2}$ and PersonSpec ${ }_{2}$-both have signature $S$-to get a specification with signature $S$, and with a restriction that is equivalent to the conjunction of the restrictions of Register and PersonSpec:

$$
\text { PersonRegister }_{2}=\text { Register }_{2} \cup \text { PersonSpec }_{2}
$$

PersonRegister $_{2}$ specifies all the components of Register and PersonSpec, but it only contains a single version of the type Person.

The above method of combining specifications is more complicated than using the sum operator which is usually more appropriate for combining specifications. Some algebraic specification languages provide an operator, called amalgamated sum, that combines specifications in a similar manner to that described above. 
To make a complete specification of a library system, we would expect to combine Catalogue $_{3}$ with either PersonRegister ${ }_{1}$ or PersonRegister ${ }_{2}$. We would also expect to add other specifications such as a loans module, for example. However, we do not pursue any of these additions.

\subsubsection{The Book Catalogue Revisited}

We may regain a specification similar to Catalogue from Catalogue 3 by using the hide operator $(\backslash)$. For example, consider the following specification:

Catalogue $_{6}=$ Catalogue $_{3} \backslash\{m k B o o k$, author, title, bookId $\}$

The visible signature of Catalogue $_{6}$ contains the components defined in Catalogue. However, Catalogue $e_{6}$ is not equal to Catalogue: the signature of Catalogue $_{6}$ contains local components and its restriction is still the conjunction of the restrictions of Catalogue and BookSpec.

The signature of Catalogue $_{6}$ contains local components because when we hide components they are not removed, but become local components. However, we can use the derive $(\downarrow)$ operator to remove components from specifications. For example, we will describe how to use derive to remove mkBook, author, title and bookId from Catalogue $_{3}$. We require the function

$$
h \in \operatorname{Sig}\left(\text { Catalogue }_{3}\right) \rightarrow \operatorname{Sig}(\text { Catalogue })
$$

defined in Figure 7.6. Function $h$ takes any computational element satisfying the signature of Catalogue $_{3}$, and removes the components mkBook, author, title and bookId to yield a computational element satisfying Sig(Catalogue). We use $h$, in conjunction with derive, to make Catalogue $_{7}$ (below) which is Catalogue $_{3}$ with the components mkBook, author, title and bookId removed:

$$
\text { Catalogue }_{7}=\text { Catalogue }_{3} \downarrow h
$$

The signature of Catalogue $_{7}$ is identical to the signature of Catalogue, and the restriction of Catalogue $_{7}$ is equivalent to the restriction of Catalogue $e_{3}$. A more technical description of Catalogue $_{7}$ is that it is a type containing all modules satisfying Catalogue $_{3}$, but with $h$ applied to their computational elements to remove the components $m k B o o k$, author, title and bookId (i.e. Catalogue ${ }_{7}$ is a type containing modules whose computational elements are in the set $\left\{h(c e(m)) \mid m \in\right.$ Catalogue $\left.\left._{3}\right\}\right)$.

Catalogue $_{6}$ and Catalogue $_{7}$ illustrate the choice we have when discarding components: components can be made local, or they can be removed. We prefer to hide components by using hide rather than remove them using derive. Firstly, hide is easier to use than derive, as hide does not require us to supply an extra function, such as $h$. Secondly, if a component is made local then it can still be used to help specify other components in a specification. In contrast, we cannot remove a component from a specification if 


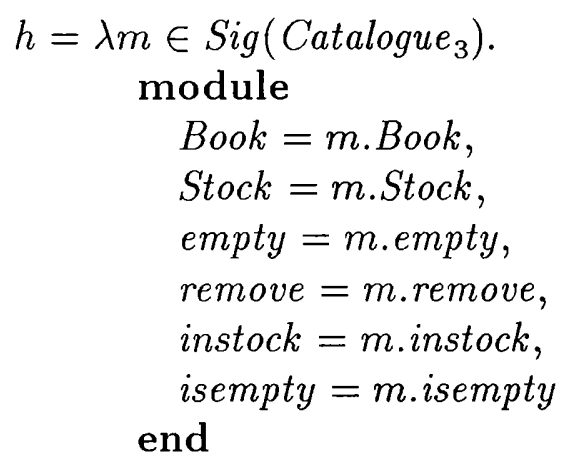

Figure 7.6: A function $h \in \operatorname{Sig}\left(\right.$ Catalogue $\left._{3}\right) \rightarrow \operatorname{Sig}($ Catalogue $)$

the component is used to give the type of other components in the signature of the specification. However, if a component is only used in the restriction of a specification, then it can be removed using derive without affecting the behaviour of the remaining components in the specification; this fact may seem surprising, but it is justified by the semantics of derive.

\subsubsection{Discussion of the Example}

We have shown how a suitable collection of specification operators can be used to systematically construct specifications. We have employed an incremental style of specification construction to make specifications for both a book catalogue and a library register. An incremental style of specification construction is particularly suited for the construction of large specifications. The operators enrich and sum play a key role in allowing us to make specifications in an incremental style. For example, we used Catalogue as an initial framework for Catalogue , $_{4}$ and most of the other parts of Catalogue $_{4}$ were added using enrich and sum. The rename operator also played a useful role by allowing us to reuse Catalogue to obtain a starting point for the construction of PersonRegister ${ }_{1}$ and PersonRegister . $_{2}$.

There are several advantages to an incremental approach to specification construction. Firstly, an incremental approach simplifies the specification task. Secondly, it is easier to modify a specification if its requirements change. For example, if we decide to change Catalogue $_{4}$ then the steps Catalogue-Catalogue ${ }_{3}$ can still be used to make a new version of Catalogue $_{4}$. Changing Catalogue ${ }_{4}$ may still require us to re-specify Catalogue $_{6}$ and Catalogue $_{7}$ which depend on Catalogue ${ }_{4}$, but this is preferable to completely re-specifying the catalogue. The individual steps in the development of a specification can also be reused to make other specifications. Typically, we only reuse

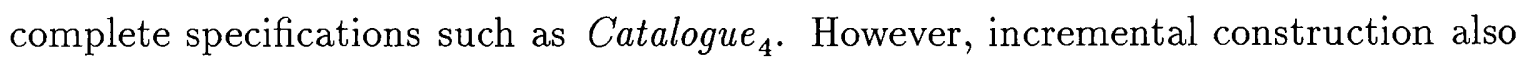
makes the intermediate steps Catalogue-Catalogue ${ }_{3}$ available for reuse. 
The library example also raises other issues. These include the way in which we choose the structure of a specification; and the use of parameterisation to construct specifications. For example, combining Catalogue with BookSpec may have been more suited to a parameterised approach in which we parameterised Catalogue with respect to BookSpec. We discuss these issues later.

In summary, we have shown how a small collection of specification operators can be used to construct specifications in an incremental style.

\subsection{The Semantics of Specification Operators}

In this section, we give the formal definitions of the specification operators. Semantically, specification operators are transformations that take a list of arguments, including specifications, and return a specification as result. The specification operators are defined in terms of the signature and computational element operators defined in the previous chapter. In the following, we will write specifications in MLT, such as $\sum m \in S . R(m)$, in the form $\langle S \mid(m) R\rangle$.

\subsubsection{Rename}

The definition of the renaming operation on specifications is given in Definition 7.1, below, with an explanation following. $S[p \backslash q]$ is signature $S$ with component $p$ renamed to $q$. Note that the restriction $R\left(m[p \backslash q]_{S}^{-1}\right)$ is expressed using the inverse computational element renaming operator $-[p \backslash q]_{S}^{-1} \in S[p \backslash q] \rightarrow S$. The restriction $R\left(m[p \backslash q]_{S}^{-1}\right)$ expresses the requirement that $m \in S[p \backslash q]$ must satisfy the restriction of $\langle S \mid(m) R\rangle$ if we rename component $q$ (in $m$ ) back to $p$. In other words, the components supplied by $\langle S \mid(m) R\rangle[p \backslash q]$ and $\langle S \mid(m) R\rangle$ must be identical except that component $p$ in $\langle S \mid(m) R\rangle$ is named $q$ in $\langle S \mid(m) R\rangle[p \backslash q]$.

Definition 7.1 (Rename)

Given any $\langle S \mid(m) R\rangle$ spec, and component names $p, q \in \mathbb{S}$ then

$$
\langle S \mid(m) R\rangle[p \backslash q]=\left\langle S[p \backslash q] \mid(m) R\left(m[p \backslash q]_{S}^{-1}\right)\right\rangle
$$

At first sight, $\langle S[p \backslash q] \mid(m) R(p \backslash q)\rangle$ seems to be an alternative definition of $\langle S \mid(m) R\rangle[p \backslash q]$ where $R(p \backslash q)$ denotes the textual substitution of component name $p$ by $q$, in $R$. However, the substitution $R(p \backslash q)$ may do more than just rename $p$ to $q$; it may actually change the intended behaviour of the specification. For example, consider the specification $S P=\langle S|(m)$ " $p$ " $=_{\mathbb{S}} m$. " $p$ " $\rangle$; we assume $S P$ supplies a component $p \in \mathbb{S}$. If we rename $p$ to $q$ using the alternative definition of renaming, 
proposed above, we get the specification $S P^{*}=\langle S[p \backslash q]|(m) " q "=_{\mathbb{S}} m$. " $q$ " $\rangle$. Note that the restriction of $S P^{*}$ expresses that the component $q$ is the string " $q$ ". This is wrong, since renaming a component must not change its behaviour, i.e. component $q$ should be specified to be the string " $p$ ". The example is contrived, but it does illustrates that the alternative definition of renaming is flawed.

Remark If $(m) R(p \backslash q)$ is equivalent to $(m) R\left(m[p \backslash q]_{S}^{-1}\right)$ with respect to weak type equality (i.e. $\left.(m) R(p \backslash q) \Leftrightarrow(m) R\left(m[p \backslash q]_{S}^{-1}\right)\right)$ then we can use $(m) R(p \backslash q)$ in place of the restriction of $\langle S \mid(m) R\rangle[p \backslash q]$. We discuss this property in Section 7.5.

\subsubsection{Hide}

We define hide in the same style as we defined rename. The definition of hide is given in Definition 7.2 with an explanation following.

Definition 7.2 (Hide)

Given any $\langle S \mid(m) R\rangle$ spec and a set of identifiers $i \in \operatorname{Set}(\mathbb{S})$ then

$$
\langle S \mid(m) R\rangle \backslash i=\left\langle S \backslash i \mid(m) R\left(m \backslash{ }_{S}^{-1} i\right)\right\rangle
$$

The expression $S \backslash i$ denotes the application of signature hiding to $S$. The expression $m \backslash{ }_{S}^{-1} i$ denotes the application of the inverse computational element hiding operator $-\backslash_{S}^{-1} i \in S \backslash i \rightarrow S$ to $m \in S \backslash i$. Viewing $\langle S \mid(m) R\rangle \backslash i$ as a type, its members are modules whose computational elements are in the set $\{c e(x) \backslash i \mid x \in\langle S \mid(m) R\rangle\}$.

It might seem reasonable to define $\langle S \mid(m) R\rangle \backslash i$ as $\langle S \backslash i \mid(m) R\rangle$. However, $R(m)$ may be ill-typed since $R(m)$ is defined under the assumption that $m \in S$, so it may not be well-typed for $m \in S \backslash i$. If $R(m)$ is well-typed for all $m \in S \backslash i$, and is equivalent to the restriction of $\langle S \mid(m) R\rangle \backslash i$ then we can use $R$ in place of the restriction of $\langle S \mid(m) R\rangle \backslash i$; this property is discussed in Section 7.5.

\subsubsection{Union}

The union operator is defined in terms of the product type constructor $(\times)$. Union uses $\times$ to combine the restrictions of two specifications:

Definition 7.3 (Union)

Given any $\left\langle S_{1} \mid(m) R_{1}\right\rangle$ spec and $\left\langle S_{2} \mid(m) R_{2}\right\rangle$ spec such that $S_{1}=S_{2}$, then

$$
\left\langle S_{1} \mid(m) R_{1}\right\rangle \cup\left\langle S_{2} \mid(m) R_{2}\right\rangle=\left\langle S_{1} \mid(m) R_{1}(m) \times R_{2}(m)\right\rangle
$$

Note that we apply $R_{2}$ to $m \in S_{1}$ even though $R_{2}$ is dependent on all $m \in S_{2}$ : we can apply $R_{2}$ to all $m \in S_{1}$ since $S_{1}=S_{2}$. 


\subsubsection{Derive}

The definition of derive is given in Definition 7.4 with justification following. $\left\langle S_{1} \mid\left(m_{1}\right) R\right\rangle \downarrow f$ (where $f \in S_{1} \rightarrow S_{2}$ ) is a type whose members are all modules $x \in\left\langle S_{1} \mid\left(m_{1}\right) R\right\rangle$ with $f$ applied to their computational elements. In other words, the members of $\left\langle S_{1} \mid\left(m_{1}\right) R\right\rangle \downarrow f$ are modules whose computational elements are in the set $\left\{f(c e(x)) \mid x \in\left\langle S_{1} \mid\left(m_{1}\right) R\right\rangle\right\}$. This set can also be expressed as:

$$
\left\{m_{2} \in S_{2} \mid \exists x \in\left\langle S_{1} \mid\left(m_{1}\right) R\right\rangle . f(c e(x))=m_{2}\right\}
$$

This set, when expressed as a type, justifies the definition of derive given below.

Definition 7.4 (Derive)

Given any $\left\langle S_{1} \mid\left(m_{1}\right) R\right\rangle$ spec, $S_{2}$ sig, and a function $f \in S_{1} \rightarrow S_{2}$, then

$$
\left\langle S_{1} \mid\left(m_{1}\right) R\right\rangle \downarrow f=\left\langle S_{2} \mid\left(m_{2}\right) \exists x \in\left\langle S_{1} \mid\left(m_{1}\right) R\right\rangle .\left[f(c e(x))=S_{S_{2}} m_{2}\right]\right\rangle
$$

\subsubsection{Translate}

The formal definition of translate is given in Definition 7.5 with explanation following. $\left\langle S_{1} \mid(m) R\right\rangle \uparrow f$ (where $f \in S_{2} \rightarrow S_{1}$ ) is a type whose members are modules, such that for all $x \in\left\langle S_{1} \mid(m) R\right\rangle \uparrow f, x$ can be transformed into a module satisfying $\left\langle S_{1} \mid(m) R\right\rangle$ by applying $f$ to the computational element of $x$. In other words, for all $x \in\left\langle S_{1} \mid(m) R\right\rangle \uparrow f$, the restriction $R(f(c e(x)))$ is true where $c e(x) \in S_{2}$ and $f(c e(x)) \in S_{1}$. The set $\left\{m \in S_{2} \mid R(f(m))\right\}$ contains the computational elements of all modules satisfying $\left\langle S_{1} \mid(m) R\right\rangle \uparrow f$. This set, when expressed as a type, justifies the definition of translate given below.

Definition 7.5 (Translate)

Given any $\left\langle S_{1} \mid(m) R\right\rangle$ spec, $S_{2}$ sig and a function $f \in S_{2} \rightarrow S_{1}$, then

$$
\left\langle S_{1} \mid(m) R\right\rangle \uparrow f=\left\langle S_{2} \mid(m) R(f(m))\right\rangle
$$

As a point of interest, both rename and hide are special cases of translate. rename can be defined using translate by choosing the inverse renaming operator on computational elements as a translate map; and hide can be defined by choosing the inverse hiding operator on computational elements as a translate map:

Fact $7.1\langle S \mid(m) R\rangle[p \backslash q]=\langle S \mid(m) R\rangle \uparrow\left(\lambda m \in S[p \backslash q] . m[p \backslash q]_{S}^{-1}\right)$

Fact $7.2\langle S \mid(m) R\rangle \backslash i=\langle S \mid(m) R\rangle \uparrow\left(\lambda m \in S \backslash i \cdot m \backslash{ }_{S}^{-1} i\right) \square$ 


\subsubsection{Sum}

The definition of the sum operator is given in Definition 7.6 with explanation following. The signature of $\left\langle S_{1} \mid(m) R_{1}\right\rangle+\left\langle S_{2} \mid(m) R_{2}\right\rangle$ is $S_{1} \oplus S_{2}$ which is the signature concatenation of $S_{1}$ and $S_{2}$. Consequently, the restriction of $\left\langle S_{1} \mid(m) R_{1}\right\rangle+\left\langle S_{2} \mid(m) R_{2}\right\rangle$ depends on computational elements with type $S_{1} \oplus S_{2}$. Note that the restriction of $\left\langle S_{1} \mid(m) R_{1}\right\rangle+\left\langle S_{2} \mid(m) R_{2}\right\rangle$ is defined using the projection functions $\begin{gathered}s_{1} s_{2} \\ \Pi_{1}\end{gathered}$ and $\begin{gathered}S_{1} s_{2} \\ \Pi_{2}\end{gathered}$. Recall that given any $m=\left(m_{1} \oplus m_{2}\right) \in S_{1} \oplus S_{2}$ where $m_{1} \in S_{1}$ and $m_{2} \in S_{2}$,

$$
\begin{aligned}
& s_{1} s_{2}(m)=m_{1} \in S_{1} \text { and } ; \\
& \Pi_{1} s_{2}(m)=m_{2} \in S_{2} \\
& \Pi_{2}(m)
\end{aligned}
$$

Let us consider the restriction of $\left\langle S_{1} \mid(m) R_{1}\right\rangle+\left\langle S_{2} \mid(m) R_{2}\right\rangle$ :

$$
(m) R_{1}\left(\begin{array}{c}
s_{1} S_{2} \\
\Pi_{1}
\end{array}(m)\right) \times R_{2}\left(\begin{array}{c}
s_{1} s_{2} \\
\Pi_{2}
\end{array}(m)\right) \text { where } m \in\left(S_{1} \oplus S_{2}\right)
$$

$s_{1}^{s_{1} S_{2}}(m) \in S_{1}$ is a computational element containing the components in $m$ that are specified by $S_{1} ;{ }_{\Pi_{2}}^{S_{1} S_{2}}(m) \in S_{2}$ is a computational element containing the components in $m$ that are specified by $S_{2}$. Therefore, $R_{1}\left(\begin{array}{c}S_{1} S_{2} \\ \Pi_{1}\end{array}(m)\right)$ expresses the requirement that the components in $m$ that are specified by $S_{1}$ must satisfy $R_{1} ; R_{2}\left(\begin{array}{c}S_{1} S_{2} \\ \Pi_{2}\end{array}(m)\right)$ expresses the requirement that the components in $m$ that are specified by $S_{2}$ must satisfy $R_{2}$.

\section{Definition 7.6 (Sum)}

Given any $\left\langle S_{1} \mid(m) R_{1}\right\rangle$ spec and $\left\langle S_{2} \mid(m) R_{2}\right\rangle$ spec then

$$
\left\langle S_{1} \mid(m) R_{1}\right\rangle+\left\langle S_{2} \mid(m) R_{2}\right\rangle=\left\langle\left( S_{1} \oplus S_{2}\left|(m) R_{1}\left(\begin{array}{c}
s_{1} S_{2} \\
\Pi_{1}
\end{array}(m)\right) \times R_{2}\left(\begin{array}{c}
s_{1} S_{2} \\
\Pi_{2}
\end{array}(m)\right)\right\rangle\right.\right.
$$

Remark It might seem that we can give a simpler definition of sum by defining the restriction of $\left\langle S_{1} \mid(m) R_{1}\right\rangle+\left\langle S_{2} \mid(m) R_{2}\right\rangle$ to be $R_{1}(m) \times R_{2}(m)\left(m \in S_{1} \oplus S_{2}\right)$. However, this restriction may be ill-typed for some restrictions $R_{1}$ and $R_{2}$. This is because $R_{1}$ and $R_{2}$ depend on computational elements of type $S_{1}$ and $S_{2}$, respectively, so both $R_{1}(m)$ and $R_{2}(m)$ may be ill-typed for some, or all, $m \in S_{1} \oplus S_{2}$. If $R_{1}(m) \times$ $R_{2}(m)$ is well-typed for all $m \in S_{1} \oplus S_{2}$ then we can use it to simplify the restriction of $\left\langle S_{1} \mid(m) R_{1}\right\rangle+\left\langle S_{2} \mid(m) R_{2}\right\rangle$; this point is discussed in Section 7.5

As a point of interest, sum can be expressed using the union and translate operations:

Theorem $7.1\left\langle S_{1} \mid(m) R_{1}\right\rangle+\left\langle S_{2} \mid(m) R_{2}\right\rangle=\left\langle S_{1} \mid(m) R_{1}\right\rangle \uparrow \underset{\Pi_{1}}{S_{1} S_{2}} \cup\left\langle S_{2} \mid(m) R_{2}\right\rangle \uparrow \stackrel{s_{1} S_{2}}{\Pi_{2}}$

\section{Proof}

$$
\begin{aligned}
& \left\langle S_{1} \mid(m) R_{1}\right\rangle+\left\langle S_{2} \mid(m) R_{2}\right\rangle \\
& =\text { "definition of sum" } \\
& \left\langle\left( S_{1} \oplus S_{2}\left|(m) R_{1}\left({ }_{\Pi_{1}}^{s_{1} s_{2}}(m)\right) \times R_{2}\left(\begin{array}{c}
s_{1} s_{2} \\
\Pi_{2}
\end{array}(m)\right)\right\rangle\right.\right. \\
& =\text { "definition of union" } \\
& \left\langle\left(S _ { 1 } \oplus S _ { 2 } | R _ { 1 } ( \begin{array} { c } 
{ { } _ { 1 } S _ { 2 } } \\
{ \Pi _ { 1 } }
\end{array} ( m ) ) \rangle \cup \left\langle\left( S_{1} \oplus S_{2}\left|(m) R_{2}\left(\begin{array}{c}
{ }_{1} S_{2} \\
\Pi_{2}
\end{array}(m)\right)\right\rangle\right.\right.\right.\right. \\
& =\text { "type of } \begin{array}{c}
s_{1} s_{2} \\
\Pi_{1}
\end{array} \text { and } \begin{array}{c}
s_{1} s_{2} \\
\Pi_{2}
\end{array} \text {, definition of translate" } \\
& \left\langle S_{1} \mid(m) R_{1}\right\rangle \uparrow{\stackrel{S}{\Pi_{1}} S_{2}}_{\Pi_{1}} \cup\left\langle S_{2} \mid(m) R_{2}\right\rangle \uparrow \stackrel{S_{1} S_{2}}{\Pi_{2}}
\end{aligned}
$$




\subsubsection{Enrich}

The formal definition of enrich is given in Definition 7.7 with an explanation following. Consider the specification $\left\langle S_{1} \mid(m) R_{1}\right\rangle \triangleleft \Lambda\left\langle S_{2} \mid R_{2}\right\rangle . S_{2}$ is a dependent signature that supplies new components to be added to $\left\langle S_{1} \mid(m) R_{1}\right\rangle$. The components supplied by $S_{2}$ may be dependent on the components of $S_{1}$, i.e. $S_{1} \vdash S_{2}$ sig holds. The signature of $\left\langle S_{1} \mid(m) R_{1}\right\rangle \triangleleft \Lambda\left\langle S_{2} \mid R_{2}\right\rangle$ is $S_{1} \oplus S_{2}$; recall that $\oplus$ allows $S_{2}$ to be dependent on $S_{1}$. The restriction $R_{2}$ depends on the components supplied by $S_{1}$ and $S_{2}$, i.e. $R_{2}$ depends on any $m \in S_{1} \oplus S_{2}$. Let us consider the restriction of $\left\langle S_{1} \mid(m) R_{1}\right\rangle \triangleleft \Lambda\left\langle S_{2} \mid R_{2}\right\rangle:$

$$
(m) R_{1}\left(\begin{array}{c}
S_{1} S_{2} \\
\Pi_{1}
\end{array}(m)\right) \times R_{2}(m) \text { where } m \in S_{1} \oplus S_{2}
$$

$R_{1}\left(\begin{array}{c}s_{1} S_{2} \\ \Pi_{1}\end{array}(m)\right)$ expresses that the components of $m$ that are specified by $S_{1}$ (i.e. the components in computational element $\left.{ }_{\Pi_{1}}^{s_{1} s_{2}}(m)\right)$ must satisfy $R_{1} . R_{2}(m)$ expresses that all the components in $m$ must satisfy $R_{2}$.

\section{Definition 7.7 (Enrich)}

Given any $\left\langle S_{1} \mid(m) R_{1}\right\rangle$ spec, a dependent signature $S_{2}$ such that $S_{1} \vdash S_{2}$ sig holds, and a restriction $R_{2}$ such that $\left[m \in\left(S_{1} \oplus S_{2}\right) \triangleright R_{2}(m)\right.$ type] then

$$
\left\langle S_{1} \mid(m) R_{1}\right\rangle \triangleleft \Lambda\left\langle S_{2} \mid R_{2}\right\rangle=\left\langle S_{1} \oplus S_{2} \mid(m) R_{1}\left(\begin{array}{c}
S_{1} S_{2} \\
\Pi_{1}
\end{array}(m)\right) \times R_{2}(m)\right\rangle
$$

The definitions of enrich and sum differ only because enrich defines $R_{2}$ to be dependent on all $m \in S_{1} \oplus S_{2}$ rather than all $m \in S_{2}$. We can express sum in terms of enrich, and enrich can be expressed in terms of the union and translate operators:

Fact 7.3 $\left\langle S_{1} \mid(m) R_{1}\right\rangle+\left\langle S_{2} \mid(m) R_{2}\right\rangle=\left\langle S_{1} \mid(m) R_{1}\right\rangle \triangleleft \Lambda\left\langle S_{2} \mid R_{2}\left(\begin{array}{c}S_{1} S_{2} \\ \Pi_{2}\end{array}(m)\right)\right\rangle$

Fact $7.4\left\langle S_{1} \mid(m) R_{1}\right\rangle \triangleleft \Lambda\left\langle S_{2} \mid R_{2}\right\rangle=\left\langle S_{1} \mid(m) R_{1}\right\rangle \uparrow \underset{\Pi_{1}}{S_{1} S_{2}} \cup\left\langle S_{1} \oplus S_{2} \mid(m) R_{2}(m)\right\rangle \square$

\subsubsection{Summary}

We have defined the formal semantics of each specification operator. We note that the definitions of some of the operators are more complicated than we might have expected.

\subsection{Unfolding Specification Operators}

In Section 7.4, we remarked that we can sometimes simplify the restrictions of specifications that are made using rename, hide or sum, and in this section we give some laws to do just that. In the following, if we say that two specifications are equivalent, we mean that they are equivalent with respect to specification equality $(\Leftrightarrow)$; 
for example, if we say $S P_{1}$ is equivalent to $S P_{2}$ we mean $S P_{1} \Leftrightarrow S P_{2}$. If we say two restrictions (or propositions) are equivalent, we mean equivalence with respect to weak type equality $\Leftrightarrow$.

We begin by considering Theorem 7.2 which shows how to simplify the restrictions of specifications that are made using rename. The pre-condition in Theorem 7.2 expresses the requirement that the textual substitution $R(p \backslash q)$ must not change the behaviour of $p$-and the other components supplied by $\langle S \mid(m) R\rangle$-after $p$ is renamed to $q$ in $R$. In Section 7.4.1, we showed that the pre-condition may not hold if $q$ is used as a string in the restriction $(m) R$. Apart from that situation, the pre-condition is true for all specifications that only use $m$ in $(m) R$ to refer to components in $m$ by dot notation. Such specifications include all canonical specifications, and all structured specifications that can be unfolded to canonical specifications.

\section{Theorem 7.2 (Unfolding Rename)}

Given $\langle S \mid(m) R\rangle$ spec and $\langle S[p \backslash q] \mid(m) R(p \backslash q)\rangle$ spec where $p, q \in \mathbb{S}$, if

$\forall m \in S . R(m) \Leftrightarrow R(p \backslash q)(m[p \backslash q])$

then

$$
\langle S \mid(m) R\rangle[p \backslash q] \Leftrightarrow\langle S[p \backslash q] \mid(m) R(p \backslash q)\rangle
$$

Proof The proof uses Lemma 7.1 which says that the precondition in Theorem 7.2 implies that the restriction of $\langle S \mid(m) R\rangle[p \backslash q]$ is equivalent to $R(p \backslash q)$. The proof is

$$
\begin{gathered}
\langle S \mid(m) R\rangle[p \backslash q] \\
=\text { "definition of rename" } \\
\left\langle S[p \backslash q] \mid(m) R\left(m[p \backslash q]_{S}^{-1}\right)\right\rangle \\
\Leftrightarrow \text { "precondition, Lemma 7.1, definitions of } \Leftrightarrow " \\
\langle S[p \backslash q] \mid(m) R(p \backslash q)\rangle
\end{gathered}
$$

Lemma 7.1 If $\forall m \in S . R(m) \Leftrightarrow R(p \backslash q)(m[p \backslash q])$ then

$$
\forall m \in S[p \backslash q] . A x(\langle S \mid(m) R\rangle[p \backslash q])(m) \Leftrightarrow R(p \backslash q)(m))
$$

\section{Proof}

$$
\begin{aligned}
& \forall m \in S . R(m) \Leftrightarrow R(p \backslash q)(m[p \backslash q]) \\
& \Rightarrow \text { " } m \in S[p \backslash q] \text { implies } m[p \backslash q]_{S}^{-1} \in S \text { " } \\
& \forall m \in S[p \backslash q] . R\left(m[p \backslash q]_{S}^{-1}\right) \Leftrightarrow R(p \backslash q)\left(\left(m[p \backslash q]_{S}^{-1}\right)[p \backslash q]\right) \\
& =\text { "- }[p \backslash q]_{S}^{-1} \text { is inverse of }-[p \backslash q] " \\
& \forall m \in S[p \backslash q] . R\left(m[p \backslash q]_{S}^{-1}\right) \Leftrightarrow R(p \backslash q)(m) \\
& =\text { "definition of rename" } \\
& \forall m \in S[p \backslash q] . A x(\langle S \mid(m) R\rangle[p \backslash q])(m) \Leftrightarrow R(p \backslash q)(m)
\end{aligned}
$$




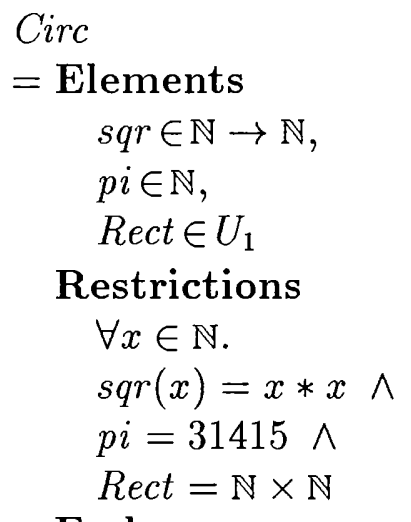

Figure 7.7: Specification Circ

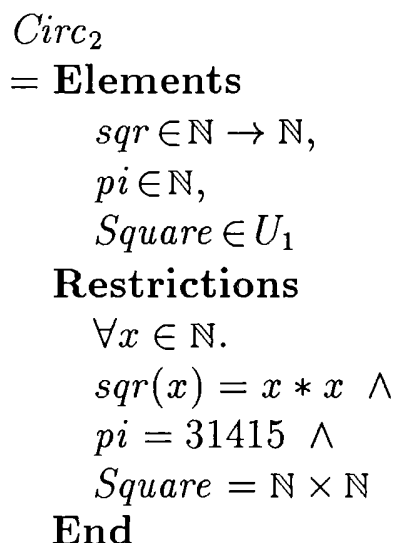

Figure 7.8: Specification $\operatorname{Circ}_{2}$

Although the pre-condition in Theorem 7.2 seems complicated, in practice, it is usually easy to discharge. For example, consider the specifications $\mathrm{Circ}$ and $\mathrm{Circ}_{2}$ given in Figure 7.7 and Figure 7.8, respectively. Circ $_{2}$ is Circ with Rect renamed to Square. We can easily verify that the signature of $\operatorname{Circ}_{2}$ equals $\operatorname{Sig}(\operatorname{Circ})[\operatorname{Rect} \backslash$ Square], and the restriction of $C_{i r c}$ equals $A x(\operatorname{Circ})(m)($ Rect $\backslash S q u a r e)$ for all $m \in \operatorname{Sig}(\operatorname{Circ})$. Figure 7.9 contains a proof that $A x(\operatorname{Circ})(m)=A x\left(\operatorname{Circ}_{2}\right)(m[$ Rect $\backslash$ Square $])$ for all $m \in \operatorname{Sig}($ Circ $)$. Hence, by Theorem 7.2 we get that $\operatorname{Circ}[$ Rect $\backslash S q u a r e] \Leftrightarrow \operatorname{Circ}_{2}$.

Theorem 7.3 is used to simplifying the restrictions of specifications constructed with a hide operation. The pre-condition in Theorem 7.3 ensures that the restrictions of $\langle S \backslash i \mid(m) R\rangle$ and $\langle S \mid(m) R\rangle \backslash i$ are logically equivalent. In practice, the pre-condition is true for all canonical specifications, and any structured specification that can be unfolded to a canonical specification.

\section{Theorem 7.3 (Unfolding Hide)}

Given $\langle S \mid(m) R\rangle$ spec and $\langle S \backslash i \mid(m) R\rangle$ spec where $i \in \operatorname{Set}(\mathbb{S})$, if $\forall m \in S . R(m) \Leftrightarrow R(m \backslash i)$ then $\langle S \mid(m) R\rangle \backslash i \Leftrightarrow\langle S \backslash i \mid(m) R\rangle$ 




Figure 7.9: Proof that $A x(\operatorname{Circ})(m)=A x\left(\operatorname{Circ}_{2}\right)(m[$ Rect $\backslash$ Square $])$

\section{Proof Similar to that of Theorem 7.2 above $\square$}

Finally in this section, we consider Theorem 7.4 which shows how to simplify the restrictions of specifications that are made using sum. The pre-condition in Theorem 7.4 says that the restrictions of $\left\langle S_{1} \mid(m) R_{1}\right\rangle+\left\langle S_{2} \mid(m) R_{2}\right\rangle$ and $\left\langle S_{1} \oplus S_{2} \mid(m) R_{1}(m) \times R_{2}(m)\right\rangle$ must be logically equivalent. In practice, the precondition is true if the arguments to sum $(+)$ are canonical specifications, or are structured specifications that can be unfolded to canonical specifications. Therefore, the pre-condition can often be discharged by inspection.

\section{Theorem 7.4 (Unfolding Sum)}

Given any $\left\langle S_{1} \mid(m) R_{1}\right\rangle$ spec, $\left\langle S_{2} \mid(m) R_{2}\right\rangle$ spec and $\left\langle S_{1} \oplus S_{2} \mid(m) R_{1} \times R_{2}\right\rangle$ spec, if $\forall m \in S_{1} \oplus S_{2} . R_{1}(m) \times R_{2}(m) \Leftrightarrow R_{1}\left(\begin{array}{c}s_{1} S_{2} \\ \Pi_{1}\end{array}(m)\right) \times R_{2}\left(\begin{array}{c}s_{1} s_{2} \\ \Pi_{2}\end{array}(m)\right)$

then

$$
\left\langle S_{1} \mid(m) R_{1}\right\rangle+\left\langle S_{2} \mid(m) R_{2}\right\rangle \Leftrightarrow\left\langle S_{1} \oplus S_{2} \mid(m) R_{1}(m) \times R_{2}(m)\right\rangle .
$$

Proof Follows immediately from the definition of specification equality $(\Leftrightarrow)$

In Theorem 7.4, the assumption $\left\langle S_{1} \oplus S_{2} \mid(m) R_{1} \times R_{2}\right\rangle$ spec implies that $(m) R_{1}(m) \times$ $R_{2}(m)$ is well typed for all $m \in S_{1} \oplus S_{2}$.

To summarise this section: we have given some laws that allow us to simplify the restrictions of structured specifications. Such laws can be used to unfold applications of the specification operators. 


\subsection{The Properties of Specification Operators}

In this section, we give some properties of the specification operators. The properties that we give are intended to be used to rearrange, and simplify, structured specifications. For example, when we implement a structured specification, we usually need to rearrange it to make it easier to refine and implement. This is often necessary because specifications are not always in a form that is suited to program development; when we construct specifications our main concern is that they are easy to make and understand. We also need a basic collection of properties about the specification operators in order to reason about structured specifications.

In Chapter 4, we stated that constructive logics do not enjoy many of the algebraic properties that classical logics do; for example, combinators such as $\wedge$ and $\vee$ are neither commutative nor associative with respect to the extensional, or intensional, type equality provided by MLT. Therefore, most of the properties of the specification operators are defined using specification equality $(\Leftrightarrow)$, rather than type equality $(=)$.

It is not our intention to give a complete set of properties of the specification operators, but rather to show that they have useful properties, and that these properties can be used to simplify and rearrange structured specifications.

\subsubsection{Properties of Rename and Hide}

Most properties of rename and hide are dependent on the properties of signature renaming and signature hiding, respectively, since rename and hide are defined using these signature operators. We begin by stating some facts about rename:

Fact 7.5 $S P[p \backslash p] \Leftrightarrow S P$

Fact 7.6 $S P[p \backslash q] \Leftrightarrow S P$, if $p$ is not in the domain of $S P$.

Fact 7.7 $S P[p \backslash q][q \backslash p] \Leftrightarrow S P$, if $p$ and $q$ are not in the domain of $S P$.

The above facts should not be surprising, since textual substitution has analogous properties. Like textual substitution, the order in which we rename components is important; for example, given any specification $S P$, and any components names $p, q$, $x$ and $y$, then $(S P[p \backslash q])[x \backslash y]$ is not always equivalent to $(S P[x \backslash y])[p \backslash q]$.

In the remainder of this section, we consider some properties of hide. One simple property of hide is that hiding an empty set of component names is equivalent to an identity operation on specifications:

Theorem 7.5 Given $S P$ spec then $S P \backslash\{\} \Leftrightarrow S P$. 
Proof

$$
\begin{aligned}
& \langle S \mid(m) R\rangle \backslash\{\} \\
= & \text { "definition of hide" } \\
& \left\langle S \backslash\{\} \mid(m) R\left(m \backslash{ }_{S}^{-1}\{\}\right)\right\rangle \\
= & \text { "Theorem } 6.11(S \backslash\{\}=S), \text { Theorem } 6.41\left(m \backslash{ }_{S}^{-1}\{\}=m\right) " \\
& \langle S \mid(m) R\rangle
\end{aligned}
$$

Fact 7.8 $S P \backslash i \Leftrightarrow S P$, if the names in $i$ are not in the domain of $S P$

We can express the composition of several consecutive applications of hide as a single application of hide:

Theorem 7.6 Given $S P$ spec then $S P \backslash i \backslash j \Leftrightarrow S P \backslash(i \cup j)$

\section{Proof}

$$
\begin{aligned}
&\langle S|(m) R \backslash \backslash i \backslash j \\
&= \text { "definition of hide" } \\
&\left\langle S \backslash i \mid(m) R\left(m \backslash{ }_{S}^{-1} i\right)\right\rangle \backslash j \\
&= \text { "definition of hide" } \\
&\left\langle S \backslash i \backslash j \mid(m) R\left(\left(m \backslash_{S \backslash i}^{-1} j\right) \backslash{ }_{S}^{-1} i\right)\right\rangle \\
&= \text { "Theorem } 6.12, \text { Theorem } 6.42\left(\left(m \backslash \backslash_{S \backslash i}^{-1} j\right) \backslash \bar{S}^{-1} i=m \backslash S_{S}^{-1}(i \cup j)\right) " \\
&=\left\langle S \backslash(i \cup j) \mid(m) R\left(m \backslash \bar{S}^{-1}(i \cup j)\right)\right\rangle \\
&= \text { definition of hide" } \\
& \quad\langle S \mid(m) R\rangle \backslash(i \cup j)
\end{aligned}
$$

Theorem 7.6 justifies the fact that the order in which we hide the components of a specification is not important:

Fact 7.9 $(S P \backslash i) \backslash j \Leftrightarrow(S P \backslash j) \backslash i$

The properties of rename and hide given above are in no way complete, but do illustrate that specifications of the form $S P[p \backslash q]$, and $S P \backslash i$, can be simplified and rearranged. In this section, we have not considered the relationship between rename and the other specification operators such as sum and union; nor have we considered the relationship between hide and the other specification operators. Such relationships will be discussed when we consider the properties of the other specification operators. 


\subsubsection{Properties of Union}

In the following, whenever we write $\left(S P_{1} \cup S P_{2}\right)$ we assume that $\operatorname{Sig}\left(S P_{1}\right)=\operatorname{Sig}\left(S P_{2}\right)$. Many of the properties of union are determined by the properties of the $(\times)$ type constructor, since union is defined in terms of $(x)$. Consequently, union is both associative and commutative since $(x)$ is associative and commutative if we use weak type equality $(\Leftrightarrow)$ to compare propositions:

Theorem 7.7 $S P_{1} \cup S P_{2} \Leftrightarrow S P_{2} \cup S P_{1}$

\section{Proof Omitted $\square$}

Theorem $7.8 \quad\left(S P_{1} \cup S P_{2}\right) \cup S P_{3} \Leftrightarrow S P_{1} \cup\left(S P_{2} \cup S P_{3}\right)$

\section{Proof Omitted $\square$}

The union operator is also idempotent since $(x)$ is idempotent with respect to weak type equality:

Fact 7.10 $S P \cup S P \Leftrightarrow S P \square$

Let us consider the relationship between union and the other specification operators. All the specification operators distribute over union:

Theorem $7.9\left(S P_{1} \cup S P_{2}\right)[p \backslash q] \Leftrightarrow\left(S P_{1}[p \backslash q]\right) \cup\left(S P_{2}[p \backslash q]\right)$

\section{Proof Omitted $\square$}

Theorem 7.10 $\left(S P_{1} \cup S P_{2}\right) \backslash i \Leftrightarrow\left(S P_{1} \backslash i\right) \cup\left(S P_{2} \backslash i\right)$

\section{Proof Omitted $\square$}

Theorem $7.11\left(S P_{1} \cup S P_{2}\right) \downarrow f \Leftrightarrow\left(S P_{1} \downarrow f\right) \cup\left(S P_{2} \downarrow f\right)$

\section{Proof Omitted $\square$}

Theorem 7.12 $\left(S P_{1} \cup S P_{2}\right) \uparrow f \Leftrightarrow\left(S P_{1} \uparrow f\right) \cup\left(S P_{2} \uparrow f\right)$

Proof

$$
\begin{gathered}
\left(\left\langle S_{1} \mid(m) R_{1}\right\rangle \cup\left\langle S_{2} \mid(m) R_{2}\right\rangle\right) \uparrow f \\
\Leftrightarrow " S_{1}=S_{2}, \text { definition of union" } \\
\left\langle S_{1} \mid(m) R_{1} \times(m) R_{2}\right\rangle \uparrow f \\
\Leftrightarrow \text { "definition of translate" } \\
\left\langle S_{1} \mid(m) R_{1}(f(m)) \times(m) R_{2}(f(m))\right\rangle \\
\Leftrightarrow \text { "S } S_{1}=S_{2}, \text { definition of union" } \\
\left\langle S_{1} \mid(m) R_{1}(f(m))\right\rangle \cup\left\langle S_{2} \mid(m) R_{2}(f(m))\right\rangle \\
\Leftrightarrow \text { "definition of translate, twice" } \\
\left\langle S_{1} \mid(m) R_{1}\right\rangle \uparrow f \cup\left\langle S_{2} \mid(m) R_{2}\right\rangle \uparrow f
\end{gathered}
$$


Theorem 7.13 $\left(S P_{1} \cup S P_{2}\right)+S P_{3} \Leftrightarrow\left(S P_{1}+S P_{3}\right) \cup\left(S P_{2}+S P_{3}\right)$

Proof Omitted $\square$

Theorem 7.14 $\left(S P_{1} \cup S P_{2}\right) \triangleleft \Lambda\langle S \mid R\rangle \Leftrightarrow\left(S P_{1} \triangleleft \Lambda\langle S \mid R\rangle\right) \cup\left(S P_{2} \triangleleft \Lambda\langle S \mid R\rangle\right)$

\section{Proof Omitted $\square$}

Given a specification that has a large number of axioms in its restriction, we can use the distributive properties, given above, to express such a specification as the union of several specifications, each of which have fewer axioms in their restriction than the original specification. For example, given a specification $S P=\langle S \mid(m) R\rangle[p \backslash q]$, we can usually express $R$ in the form $R_{1} \times R_{2}$ where $R_{1}$ and $R_{2}$ are propositions. Consequently, by the definition of union, and by Theorem 7.9, we can express $S P$ as

$$
\left(\left\langle S \mid(m) R_{1}\right\rangle[p \backslash q]\right) \cup\left(\left\langle S \mid(m) R_{2}\right\rangle[p \backslash q]\right)
$$

The above rearrangement of $S P$ allows us to reason about the properties of $S P$ by considering the specifications $\left\langle S \mid(m) R_{1}\right\rangle[p \backslash q]$ and $\left\langle S \mid(m) R_{2}\right\rangle[p \backslash q]$ in relative isolation. For example, we will see later that we can decompose the task of refining specifications of the form $S P_{1} \cup S P_{2}$ to that of refining $S P_{1}$ and $S P_{2}$.

\subsubsection{Properties of Sum and Enrich}

The properties of sum and enrich are dependent on the properties of signature concatenation and the $(x)$ type constructor, since we use them to define sum and enrich.

We begin by considering the properties of sum. The sum operator is associative, and this fact follows from the fact that signature concatenation is associative:

Theorem $7.15 \quad\left(S P_{1}+S P_{2}\right)+S P_{3} \Leftrightarrow S P_{1}+\left(S P_{2}+S P_{3}\right)$

Proof Given in Figure 7.10

Unlike union, the sum operator is neither commutative nor idempotent since signature concatenation is neither commutative nor idempotent.

The rename operator distributes over sum provided that the name of the component being renamed does appear in the domain of both the arguments of sum:

Theorem 7.16 if $p$ is not in the domains of $S P_{1}$ and $S P_{2}$ then

$$
\left(S P_{1}+S P_{2}\right)[p \backslash q] \Leftrightarrow\left(S P_{1}[p \backslash q]\right)+\left(S P_{2}[p \backslash q]\right)
$$

Proof Omitted $\square$ 


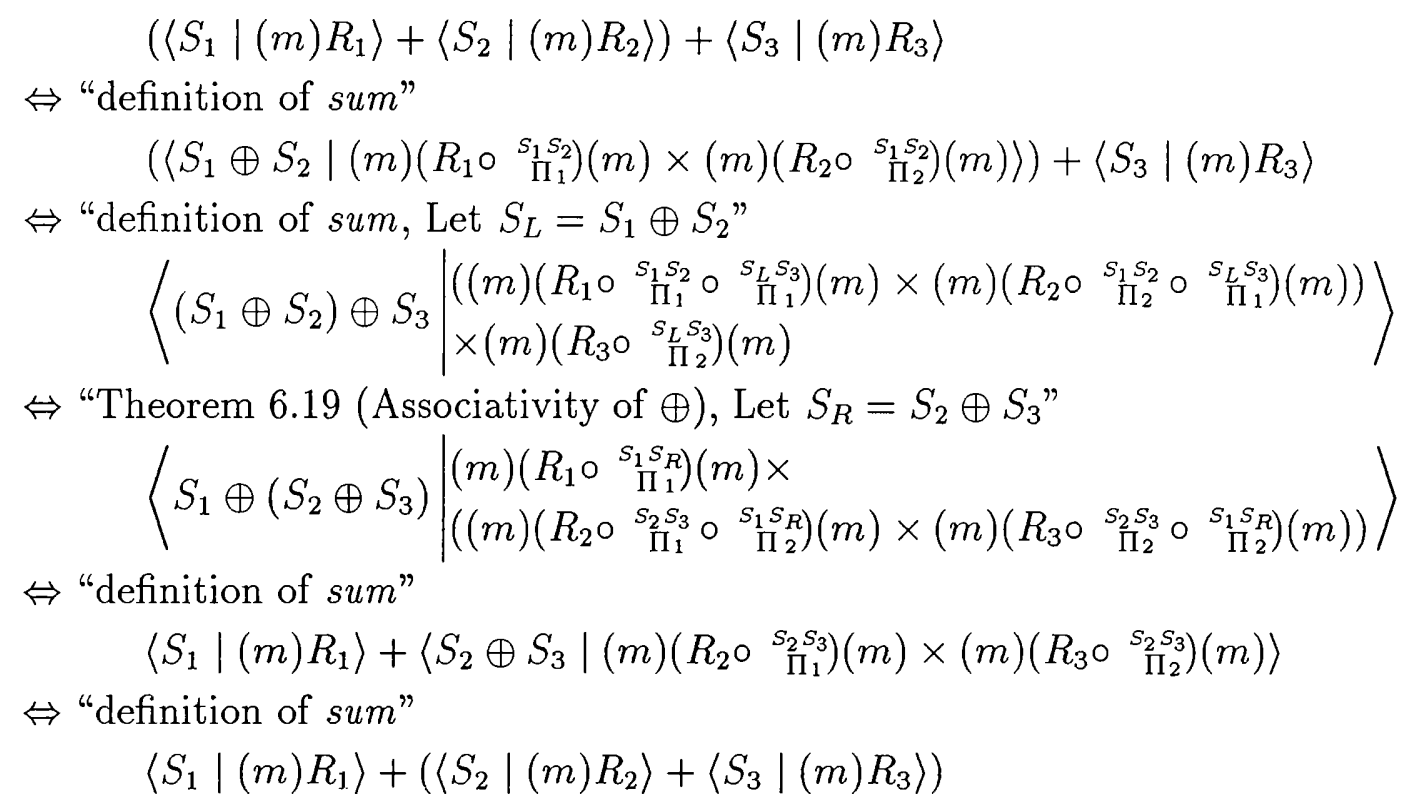

Figure 7.10: Proof of Theorem 7.15 (sum associativity)

Let us consider the precondition in Theorem 7.16. If $p$ appears in the domains of $S P_{1}$ and $S P_{2}$ then it is overloaded in $S P_{1}+S P_{2}$. Consequently, the component $p$ supplied by $S P_{1}$ is renamed in $\left(S P_{1}+S P_{2}\right)[p \backslash q]$, but not the component $p$ supplied by $S P_{2}$; recall that an attempt to rename a component that has an overloaded name only renames the first component with that overloaded name. So, the name $q$ appears once in the domain of $\left(S P_{1}+S P_{2}\right)[p \backslash q]$. In contrast, the component $p$ supplied by $S P_{1}$, and the component $p$ supplied by $S P_{2}$, are renamed in $\left(S P_{1}[p \backslash q]\right)+\left(S P_{2}[p \backslash q]\right)$. So, the name $q$ appears twice in the domain of $\left(S P_{1}[p \backslash q]\right)+\left(S P_{2}[p \backslash q]\right)$. Hence, $\left(S P_{1}+S P_{2}\right)[p \backslash q]$ is not equivalent to $\left(S P_{1}[p \backslash q]\right)+\left(S P_{2}[p \backslash q]\right)$ if $p$ appears in the domain of $S P_{1}$ and $S P_{2}$.

The hide operator distributes over the sum operator:

Theorem 7.17 $\left(S P_{1}+S P_{2}\right) \backslash i \Leftrightarrow\left(S P_{1} \backslash i\right)+\left(S P_{2} \backslash i\right)$

\section{Proof Omitted $\square$}

The derive and translate operators rarely distribute over the sum of two specifications. If they do, then the derive map and translate map used by derive and translate, respectively, have a special form:

Fact 7.11 Let $f=\lambda m \cdot f_{1}\left(\begin{array}{c}S_{1} S_{2} \\ \Pi_{1}\end{array}(m)\right) \oplus f_{2}\left(\begin{array}{c}s_{1} S_{2} \\ \Pi_{2}\end{array}(m)\right) \in\left(S_{1} \oplus S_{2}\right) \rightarrow\left(S_{3} \oplus S_{4}\right)$ where $f_{1} \in S_{1} \rightarrow S_{3}$ and $f_{2} \in S_{2} \rightarrow S_{4}$.

$$
\left(\left\langle S_{1} \mid(m) R_{1}\right\rangle+\left\langle S_{2} \mid(m) R_{2}\right\rangle\right) \downarrow f \Leftrightarrow\left\langle S_{1} \mid(m) R_{1}\right\rangle \downarrow f_{1}+\left\langle S_{2} \mid(m) R_{2}\right\rangle \downarrow f_{2}
$$


Fact 7.12 Let $f=\lambda m \cdot f_{1}\left(\begin{array}{c}S_{3} S_{4} \\ \Pi_{1}\end{array}(m)\right) \oplus f_{2}\left(\begin{array}{c}S_{3} S_{4} \\ \Pi_{2}\end{array}(m)\right) \in\left(S_{3} \oplus S_{4}\right) \rightarrow\left(S_{1} \oplus S_{2}\right)$ where $f_{1} \in S_{3} \rightarrow S_{1}$ and $f_{2} \in S_{4} \rightarrow S_{2}$.

$$
\left(\left\langle S_{1} \mid(m) R_{1}\right\rangle+\left\langle S_{2} \mid(m) R_{2}\right\rangle\right) \uparrow f \Leftrightarrow\left\langle S_{1} \mid(m) R_{1}\right\rangle \uparrow f_{1}+\left\langle S_{2} \mid(m) R_{2}\right\rangle \uparrow f_{2}
$$

In practice, derive maps rarely have the same form as $f$ in Theorem 7.11; similarly, translate maps rarely have the same form as $f$ in Theorem 7.12. However, if we regard the equivalences given in Theorem 7.11 and 7.12 as rewrite rules, then these equivalences can always be applied in the right-to-left direction to transform specifications of the form $\left(S P_{1} \downarrow f_{1}\right)+\left(S P_{2} \downarrow f_{2}\right)$ into equivalent specifications of the form $\left(S P_{1}+S P_{2}\right) \downarrow f$.

In the remainder of this section, we consider a few properties of enrich. The most common rearrangement of a specification of the form $\left\langle S_{1} \mid(m) R_{1}\right\rangle \triangleleft \Lambda\left\langle S_{2} \mid R_{2}\right\rangle$ is simply to unfold the application of enrich to get an identical specification of the form $\left\langle S_{1} \oplus S_{2} \mid(m)\left(R_{1} \circ \begin{array}{c}S_{1} S_{2} \\ \Pi_{1}\end{array}\right)(m) \times R_{2}(m)\right\rangle$. The unfolded specification can then be rearranged further, if required. We can use Fact 7.13, below, to unfold an application of enrich that adds new components to a specification, but no new restrictions. We can use Fact 7.14, below, to unfold an application of enrich that adds new restrictions to a specification, but no new components.

Fact 7.13 $\left\langle S_{1} \mid(m) R_{1}\right\rangle \triangleleft \Lambda\left\langle S_{2} \mid T\right\rangle \Leftrightarrow\left\langle S_{1} \oplus S_{2} \mid(m)\left(R_{1} \circ \underset{\Pi_{1}}{S_{1} S_{2}}\right)(m)\right\rangle$

Fact $7.14\left\langle S_{1} \mid(m) R_{1}\right\rangle \triangleleft \Lambda\left\langle\Phi \mid R_{2}\right\rangle \Leftrightarrow\left\langle S_{1} \mid(m) R_{1}(m) \times R_{2}(m)\right\rangle$

The enrich operator enjoys a kind of associativity, whereby consecutive enrichments to a specification can be combined into a single enrichment:

Theorem 7.18 Let $L=\operatorname{Sig}(S P) \oplus S_{2}$. $\left(S P \triangleleft \Lambda\left\langle S_{2} \mid R_{2}\right\rangle\right) \triangleleft \Lambda\left\langle S_{3} \mid R_{3}\right\rangle \Leftrightarrow S P \triangleleft \Lambda\left\langle S_{2} \oplus S_{3} \mid(m)\left(R_{2} \circ{ }_{\Pi_{1}}^{L S_{3}}\right)(m) \times R_{3}\right\rangle$

Proof

$$
\begin{aligned}
& \left(\left\langle S_{1} \mid(m) R_{1}\right\rangle \triangleleft \Lambda\left\langle S_{2} \mid R_{2}\right\rangle\right) \triangleleft \Lambda\left\langle S_{3} \mid R_{3}\right\rangle \\
& \Leftrightarrow \text { "definition of enrich" } \\
& \left\langle S_{1} \oplus S_{2} \mid(m)\left(R_{1} \circ \begin{array}{c}
S_{1} S_{2} \\
\Pi_{1}
\end{array}\right)(m) \times R_{2}(m)\right\rangle \triangleleft \Lambda\left\langle S_{3} \mid R_{3}\right\rangle \\
& \Leftrightarrow \text { "definition of enrich, Let } L=S_{1} \oplus S_{2} \text { " } \\
& \left\langle\left(S_{1} \oplus S_{2}\right) \oplus S_{3} \mid(m)\left(\left(R_{1} \circ \stackrel{S_{1} S_{2}}{\Pi_{1}}{ }_{\Pi_{1}}^{L S_{3}}\right)(m) \times\left(R_{2} \circ{ }_{\Pi_{1}}^{L S_{3}}\right)(m)\right) \times R_{3}(m)\right\rangle \\
& \Leftrightarrow \text { "associativity of } \oplus \text {, associativity of } \times \text {, Let } R=S_{2} \oplus S_{3} \text { " } \\
& \left\langle S_{1} \oplus\left(S_{2} \oplus S_{3}\right) \mid(m)\left(R_{1} \circ \underset{\Pi_{1}}{S_{1}}\right)(m) \times\left(\left(R_{2} \circ \underset{\Pi_{1}}{L L_{3}}\right)(m) \times R_{3}(m)\right)\right\rangle \\
& \Leftrightarrow \text { "definition of enrich" } \\
& \left\langle S_{1} \mid(m) R_{1}\right\rangle \triangleleft \Lambda\left\langle S_{2} \oplus S_{3} \mid(m)\left(R_{2} \circ \underset{\Pi_{1}}{L S_{3}}\right)(m) \times R_{3}(m)\right\rangle
\end{aligned}
$$


Both the rename and hide operators distribute over applications of enrich:

Fact 7.15 If name $p$ is not in the domains of $S P$ and $S$ then:

$$
(S P \triangleleft \Lambda\langle S \mid R\rangle)[p \backslash q] \Leftrightarrow(S P[p \backslash q]) \triangleleft \Lambda\left\langle S[p \backslash q] \mid(m) R\left(m[p \backslash q]_{S_{1} \oplus S_{2}}^{-1}\right)\right\rangle
$$

Fact 7.16 $(S P \triangleleft \Lambda\langle S \mid R\rangle) \backslash i \Leftrightarrow(S P \backslash i) \triangleleft \Lambda\left\langle S \backslash i \mid(m) R\left(m \backslash \bar{S}_{1}^{-1} \Phi_{2} i\right)\right\rangle \square$

Remark The precondition in Fact 7.15 is needed for the same reasons as we gave for requiring a similar precondition in Theorem 7.16-recall that Theorem 7.16 says that rename distributes over sum

Apart from rename and hide, the other specification operators rarely distribute over the enrichment of a specification. However, this is not a problem as, in practice, we usually only require to distribute rename and hide over enrich.

\subsubsection{Properties of Derive and Translate}

We begin by giving some properties of derive. The first property we consider is that an application of derive, using an identity function as a derive map, is equivalent to an identity operation on specifications:

Theorem 7.19

Let $I d=\lambda x . x \in \operatorname{Sig}(S P) \rightarrow \operatorname{Sig}(S P) . S P \downarrow I d \Leftrightarrow S P$.

\section{Proof Omitted}

We can express two, or more, consecutive applications of derive as a single application of derive:

\section{Theorem 7.20}

$(S P \downarrow f) \downarrow g \Leftrightarrow S P \downarrow(g \circ f)$ where $S P$ spec, $S_{2}$ sig, $S_{3}$ sig, $f \in \operatorname{Sig}(S P) \rightarrow S_{2}$ and $g \in S_{2} \rightarrow S_{3}$.

\section{Proof Omitted $\square$}

We now consider some properties of translate. Many of the properties of translate are similar to those of derive. For example, Theorem 7.21 (below) says that an application of translate, using an identity function as a translate map, is equivalent to an identity operation on specifications. Note that the equivalence expressed in Theorem 7.21 is a type equality $(=)$.

Theorem 7.21 Let $I d_{S}=\lambda m . m \in S \rightarrow S .\langle S \mid(m) R\rangle \uparrow I d_{S}=\langle S \mid(m) R\rangle$

Proof $\langle S \mid(m) R\rangle \uparrow I d_{S}=\left\langle S \mid(m) R\left(I d_{S}(m)\right)\right\rangle=\langle S \mid(m) R\rangle \square$ 
Several consecutive applications of translate can be expressed as a single application of translate:

\section{Theorem 7.22}

Given any $\left\langle S_{1} \mid(m) R_{1}\right\rangle$ spec, $S_{1}$ sig, $S_{2}$ sig, $S_{3}$ sig, $f \in S_{2} \rightarrow S_{1}$ and $g \in S_{3} \rightarrow S_{2}$,

$$
\left(\left\langle S_{1} \mid(m) R_{1}\right\rangle \uparrow f\right) \uparrow g=\left\langle S_{1} \mid(m) R_{1}\right\rangle \uparrow(f \circ g)
$$

\section{Proof}

$$
\begin{gathered}
\left(\left\langle S_{1} \mid(m) R_{1}\right\rangle \uparrow f\right) \uparrow g \\
=\text { "definition of translate" } \\
\left\langle S_{2} \mid(m)\left(R_{1} \circ f\right)(m)\right\rangle \uparrow g \\
=\text { "definition of translate" } \\
\left\langle S_{2} \mid(m)\left(\left(R_{1} \circ f\right) \circ g\right)(m)\right\rangle \\
=\text { "associativity of function composition" } \\
\left\langle S_{2} \mid(m)\left(R_{1} \circ(f \circ g)\right)(m)\right\rangle \\
=\text { "definition of translate" } \\
\left\langle S_{1} \mid(m) R_{1}\right\rangle \uparrow(f \circ g)
\end{gathered}
$$

The following fact is justified by Theorems 7.22 and by Theorem 7.21:

Fact 7.17 Given $S_{2}$ sig and $f \in \operatorname{Sig}(S P) \rightarrow S_{2}$ then $(S P \uparrow f) \uparrow f^{-1}=S P$

The translate operator is an inverse for derive, but derive is only an inverse for translate under certain conditions:

Fact 7.18 $(S P \downarrow f) \uparrow f \Leftrightarrow S P$ where $f \in \operatorname{Sig}(S P) \rightarrow S_{2}$ and $S_{2}$ sig $\square$

Fact 7.19 If $f^{-1}$ exists then $(S P \uparrow f) \downarrow f \Leftrightarrow S P$ where $f \in S_{2} \rightarrow \operatorname{Sig}(S P)$ and $S_{2}$ sig

\subsection{Discussion and Conclusion}

Several of our specification operators can be defined differently. For example, both rename and hide can be defined in terms of derive:

$$
\begin{aligned}
& S P[p \backslash q] \Leftrightarrow S P \downarrow(\lambda m \in \operatorname{Sig}(S P) . m[p \backslash q]) \\
& S P \backslash i \quad \Leftrightarrow S P \downarrow(\lambda m \in \operatorname{Sig}(S P) . m \backslash i)
\end{aligned}
$$

However, the disadvantage of using derive to define rename and hide is that the restrictions of $S P \downarrow(\lambda m \in \operatorname{Sig}(S P) . m[p \backslash q])$ and $S P \downarrow(\lambda m \in \operatorname{Sig}(S P) . m \backslash i)$ are more complicated than those of $S P[p \backslash q]$ and $S P \backslash i$. Consequently, constructive proofs concerning specifications of the form $S P[p \backslash q]$ and $S P \backslash i$ are easier to make than those concerning $S P \downarrow(\lambda m \in \operatorname{Sig}(S P) . m[p \backslash q])$ and $S P \downarrow(\lambda m \in \operatorname{Sig}(S P) . m \backslash i)$. 
One of the issues raised when making the specifications Catalogue $_{6}$ and Catalogue $_{7}$ is that of eliminating redundant components from specifications: we illustrated that redundant components can be hidden using hide, or removed using derive. In the $\mathrm{Z}$ specification language, the schema hiding operator removes components from the signature of a schema since $\mathrm{Z}$ does not allow local components. The use of derive to remove components from a specification can be shown to be similar, in function, to the use of schema hiding in Z. Z does not allow dependencies between components in a schema signature. Therefore, we can remove components from the signature of a schema without making it ill-defined. In contrast, if we remove a component from one of our specifications, then there is a possibility that the specification may become ill-defined since the type of other components in the specification may depend on the component that we remove. Hence, we prefer to hide components, rather than remove them, since hidden components remain available for use in the signature of a specification.

Some specification languages, such as $\mathrm{Z}$ and ASL, do not permit component names to be overloaded, and they resolve name-clashes-that can arise when two specifications are combined - by merging components that have the same name into a single component. One advantage of merging components is that if we intended the components to be equal then we do not have to explicitly specify that fact. However, the disadvantage of merging components is that we can accidentally merge two components that are intended to be different, as it is easy to loose track of the component names in a large specification. With our sum operator there is no danger of accidentally merging component names; if we wish to specify two components as being equal, then we can add that requirement, as an extra restriction, to a specification.

In summary, we have developed a collection of specification operators which combine, and modify specifications within our type-theoretic framework. We have shown that these specification operators facilitate the incremental construction of specifications. We have also shown that the specification operators enjoy some useful algebraic properties that can be used to change the structure of structured specifications. 


\section{Chapter 8}

\section{Refinement}

\subsection{Introduction}

The design and construction of specifications is only one half of the programming task. The other half is the development of programs that satisfy our specifications. It is now well known that specifications can play an active role in programming: it is possible, at least in principle, to derive a program from its specification by mathematical transformation. The process of mathematically transforming a specification towards a program is called refinement. In this chapter, we give a type-theoretic definition of refinement for specifications, and to use it to develop a collection of refinement laws.

Refining a specification may be regarded as the task of making concrete design decisions about properties of the specification that were left open by the specifier-the choice of data structures and error messages for example. The definition of refinement given here is one where we add more detail to a specification to get a refinement. In the context of program development in type theory, we regard refinement as being different from implementation. Specifications are types, and any refinement of a specification is a specification and, hence, a type. However, implementations are values of a type, so that implementation is the task of finding values of a type. We don't implement specifications in one go, instead we proceed to an implementation by refining a specification. Implementations become easier the more we refine a specification, and so we keep refining a specification until we arrive at a specification that strongly suggests an implementation. Then we proceed by finding an implementation of the refined specification by using a constructive proof. This last step is usually clerical and so we do not discuss it in much detail here. We refer the reader interested in deriving programs via constructive proofs to $[3,44]$.

Our definition of refinement in type theory is similar to the notion of subtyping in programming languages such as Quest [7]. Indeed, a subtype of a specification 
is a refinement. However, using subtyping as a definition of refinement is overly restrictive, as it fails to meet the following two requirements. Firstly, we want to be able to refine specifications by adding, or removing components from their signature; for example, we often add extra components that we use to refine other components in a specification. Secondly, we want to be able to refine a specification by changing the type of some of its components, as this often leads to more efficient implementations; such refinements are sometimes called data refinements. The definition of refinement that we will propose allows both kinds of refinements mentioned above. Our definition of refinement is similar to the deliverables approach of Burstall et al [6], and to refinement in ECC [27]; we discuss this related work later.

To be useful, refinement must proceed in a piecewise manner. In other words we want to refine a specification by refining its individual parts relatively independently of each other, and then combine its refined parts to get a refinement of the whole specification. A piecewise approach has several advantages. Firstly, we avoid the work of reshaping a specification before we refine it. Secondly, we can more easily reuse refinements when part of a specification is reused. Thirdly, if we change some parts of a specification after it is refined, then only the changed parts need to be re-refined to get a refinement of the new specification. A piecewise approach to refinement may not always produce the most efficient implementation of a specification. Nevertheless, it is a systematic approach to tackling the refinement of large specification.

\subsection{Refinement of Types}

In many program development formalisms--such as algebraic specifications [50, 56], and the refinement calculus [37] - a specification $P$ is refined by a specification $Q$ whenever all the implementations of $Q$ are also implementations of $P$. If we were to adopt a similar definition of refinement for type theory, then we might say that a type $P$ is refined by a type $Q$ whenever all the members of $Q$ are also members of $P$; in other words, $Q$ is a subtype of $P$. In practice, using subtyping as a definition of refinement is of limited use in type theory. For example, when we refine a specification, we often add extra components to its signature, which play an intermediary role in helping to make the refinement. However, if we use subtyping as the definition of refinement, then we cannot refine a specification by adding extra components to its signature: the implementations of the refinement will contain extra components, and so, will no longer be implementations of the original specification.

We propose a more flexible definition of refinement which allow the refinements of a type to be more than just subtypes. Using our proposed definition of refinement, if a type $P$ is refined by a type $Q$, then the values of $Q$ need not have the same 'shape' as the values of $P$. Central to our proposal is the introduction of a refinement map 
that translates values of type $Q$ to values of type $P$. We write that type $P$ is refined by type $Q$, with respect to a refinement map $f$, as $P \sqsubseteq^{f} Q$. We propose the following definition of refinement:

\section{Definition 8.1 (General Refinement)}

Given $P$ type and $Q$ type, then $P$ is refined by $Q$ with respect to $f$ (written $P \complement^{f} Q$ ) iff we have a proof of the judgement $f \in Q \rightarrow P$.

The intuition behind the definition of general refinement is that if we have a refinement map $f \in Q \rightarrow P$, then given any implementation $q$ of $Q$ (i.e. $q \in Q$ ) we can always guarantee that $f(q)$ is an implementation of the original specification $P$ (i.e. $f(q) \in P)$. For example, if $Q$ is a specification that supplies all the components that $P$ does, plus some extra components, then we would choose $f$ to be a function that takes a module $q \in Q$, and throws away the extra components in $q$ to get a module satisfying $P$. If we view $P$ and $Q$ as propositions, then the requirement that there exists some $f \in Q \rightarrow P$ says that $Q$ implies $P$; this implication corresponds with the intuition that refinement strengthens the constraints on a specification.

Refinement maps are similar, in function, to retrieve functions used in VDM data reification [21], and to functional abstraction invariants for data refinement in the refinement calculus [36,38], and to constructors used in the constructor implementation of algebraic specifications [50]. A discussion of the relationship between our definition of refinement, and those cited above, is given later.

One problem with general refinement is that its very simplicity often leads to very complicated refinement maps, especially when refining complex types such as specifications. It is more convenient to define specialised definitions of refinement for types such as specifications, under the condition that the specialised definitions are special cases of general refinement. This is not to say general refinement is not needed; it is, but mainly for refining types within specifications. For example, we would use general refinement to refine a function type that occurs in the signature of a specification. Our main interest is the refinement and development of specifications, and in the following section we give a specialised definition of refinement for specifications.

\subsection{Refinement of Specifications}

In this section we define the notion of specification refinement which is a special case of general refinement. We motivate the need for specification refinement as follows. Suppose $S P_{1} \sqsubseteq{ }^{f} S P_{2}$ where $S P_{1}$ and $S P_{2}$ are specifications and $f \in S P_{2} \rightarrow S P_{1}$. In mapping each $m \in S P_{2}$ to $f(m) \in S P_{1}$, the refinement map $f$ does two things. Firstly, it maps the witness of each $m \in S P_{2}$ to a witness of an implementation of 
$S P_{1}$, thus proving that the restriction of $S P_{2}$ implies the restriction of $S P_{1}$. Secondly, it maps the computational element of each $m \in S P_{2}$ to a computational element of type $\operatorname{Sig}\left(S P_{1}\right)$. The fact that the refinement map has two roles when refining specifications can make the refinement map difficult to construct and understand. Therefore, we propose a specialised definition of refinement for specifications which, instead of requiring a single refinement map $f \in S P_{2} \rightarrow S P_{1}$, requires the existence of two maps: one maps between witnesses, and the other between computational elements. A formal definition of specification refinement is given in Definition 8.2 with a justification of the definition following.

Definition 8.2 (Specification Refinement) Given $S P_{1}$ spec and $S P_{2}$ spec, an abstraction map between $S P_{1}$ and $S P_{2}$ is any function

$$
h \in \operatorname{Sig}\left(S P_{2}\right) \rightarrow \operatorname{Sig}\left(S P_{1}\right)
$$

such that there exists some witness $p$ that satisfies the following proposition:

$$
p \in \forall x \in \operatorname{Sig}\left(S P_{2}\right) . A x\left(S P_{2}\right)(x) \Rightarrow A x\left(S P_{1}\right)(h(x))
$$

We call $p$ a proof map. If $h$ is an abstraction map between $S P_{2}$ and $S P_{1}$ then we say that $S P_{1}$ is refined to $S P_{2}$ with respect to $h$, written $S P_{1} \sqsubseteq_{h} S P_{2}$

Notation For any specifications $S P_{1}$ and $S P_{2}$, and function $h \in \operatorname{Sig}\left(S P_{2}\right) \rightarrow$ $\operatorname{Sig}\left(S P_{1}\right)$ we will often represent $\forall x \in \operatorname{Sig}\left(S P_{2}\right) . A x\left(S P_{2}\right)(x) \Rightarrow A x\left(S P_{1}\right)(h(x))$ by $\operatorname{Res}\left(S P_{1}, S P_{2}, h\right)$ for convenience of presentation

Notation (Simple Refinement) If $\operatorname{Sig}\left(S P_{1}\right)=\operatorname{Sig}\left(S P_{2}\right)$ and $I d \in \operatorname{Sig}\left(S P_{2}\right) \rightarrow$ $\operatorname{Sig}\left(S P_{1}\right)$ is the identity function then we abbreviate $S P_{1} \sqsubseteq_{I d} S P_{2}$ to $S P_{1} \sqsubseteq S P_{2}$. The relation $\sqsubseteq$ is called the simple refinement relation.

The definition of specification refinement is justified as follows. The existence of a proof map $p$ proves that the restriction of $S P_{2}$ implies the restriction of $S P_{1}$. Proof map $p$ also proves that abstraction map $h$ maps any computational element of a module satisfying $S P_{2}$ to a computational element of a module satisfying $S P_{1}$.

If $S P_{1} \sqsubseteq_{h} S P_{2}$ than any implementation $m$ of $S P_{2}$ (i.e. $m \in S P_{2}$ ) gives an implementation of $S P_{1}$ :

Fact 8.1 If there exists some proof map $p$ that witnesses that $S P_{1} \sqsubseteq_{h} S P_{2}$ then given any $m \in S P_{2}$ :

$$
\langle h(c e(m)), p(c e(m))(p f(m))\rangle \in S P_{\mathbf{1}}
$$

where

$$
\begin{array}{lll}
p & \in & \operatorname{Res}\left(S P_{1}, S P_{2}, h\right) \\
h(c e(m)) & \in & \operatorname{Sig}\left(S P_{1}\right) ; \text { and } \\
p(c e(m))(p f(m)) & \in & \operatorname{Ax}\left(S P_{1}\right)(h(c e(m))
\end{array}
$$




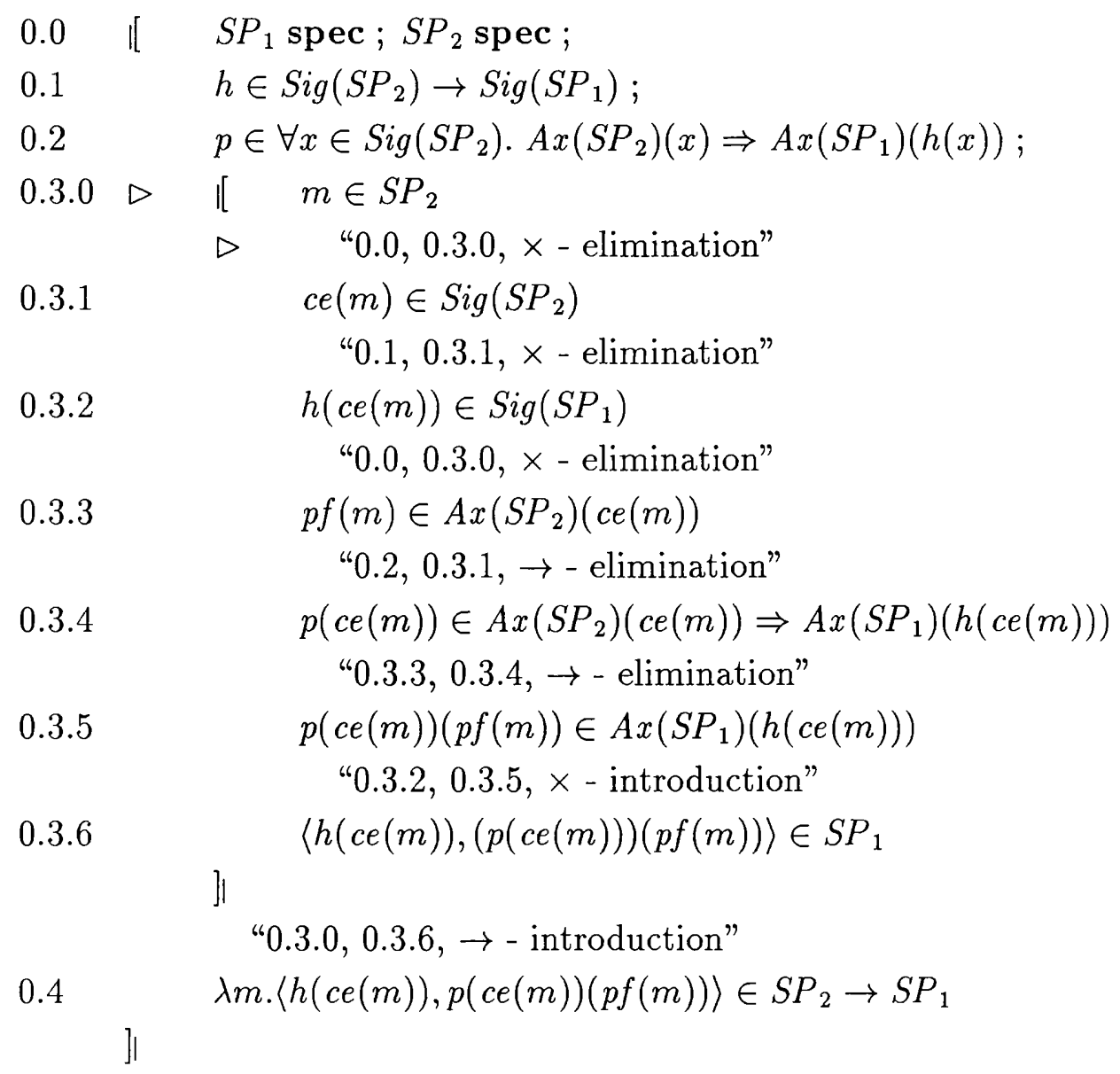

Figure 8.1: Proof of Theorem 8.1

If a specification $S P_{1}$ can be refined to some specification $S P_{2}$ using specification refinement, then $S P_{1}$ can also refined to $S P_{2}$ using general refinement:

\section{Theorem 8.1}

If $S P_{1} \sqsubseteq_{h} S P_{2}$ then $S P_{1} \sqsubseteq^{f} S P_{2}$; here $f=\lambda m .\langle h(c e(m)), p(c e(m))(p f(m))\rangle \in S P_{2} \rightarrow$ $S P_{1}$ where $p \in \operatorname{Res}\left(S P_{1}, S P_{2}, h\right)$ is a proof map.

Proof The proof is given in Figure 8.1.

The fact that a specification $S P_{1}$ can be refined to $S P_{2}$ under general refinement does not guarantee that $S P_{1}$ refines to $S P_{2}$ under specification refinement. For example, consider the following specifications

\section{$S P_{1}=$ Elements $P \in U_{1}$ Restrictions $P={ }_{U_{1}} \mathbb{N}$ end \\ $S P_{2}=$ Elements $P \in\{\mathbb{N}\}_{U_{1}}$ Restrictions $T$ end}

Under general refinement, $S P_{1} \sqsubseteq^{f} S P_{2}$ for $f=\lambda m .\left\langle h(c e(m), e q\rangle \in S P_{2} \rightarrow S P_{1}\right.$. However, $S P_{1}$ does not refine to $S P_{2}$ under specification refinement since the restriction of $S P_{2}$ does not imply the restriction of $S P_{1}$. In practice, we rarely make refinements by weakening a restriction, but if we do, then we need to use general refinement, as 
exemplified above. Most refinements needed for program development can be made using specification refinement. As we will see later, specification refinement possesses some useful properties that general refinement does not.

\subsubsection{An Example of a Specification Refinement}

In this section, we give a small example of a specification refinement. Consider the following specification of a module containing two functions $f$ and $g$ :

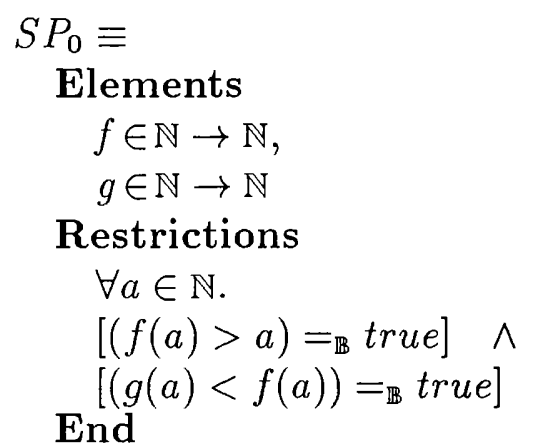

One possible refinement of $S P_{0}$ is given by the specification $S P_{1}$ :



Note the addition of an extra component $c$ in the signature of $S P_{1}$. New components, such as $c$, often play an intermediary role during refinement; for example, they may be used to break up the specification of complicated operations. Although the signature of $S P_{1}$ differs from the signature of $S P_{0}$, we can relate the two signatures by the following abstraction map:

$$
h=\lambda m . \text { module } f=m . f, g=m . g \text { end } \in \operatorname{Sig}\left(S P_{1}\right) \rightarrow \operatorname{Sig}\left(S P_{0}\right)
$$

Function $h$ returns a computational element with signature $\operatorname{Sig}\left(S P_{0}\right)$ by removing the constant component $c$ from a computational element with signature $\operatorname{Sig}\left(S P_{1}\right)$. We may observe that, for all $f, g$ and $c$, the conjunction of (4) and (5) implies (2); and (3) implies (1). Hence, the restriction of $S P_{1}$ implies the restriction of $S P_{0}$, and proof object $p$, below, witnesses this implication:

$$
p=\lambda m . \lambda x . \lambda a .\langle e q,\langle e q, e q\rangle\rangle \in \operatorname{Res}\left(S P_{0}, S P_{1}, h\right)
$$


Therefore, by the definition of specification refinement, we get that $S P_{0} \sqsubseteq_{h} S P_{1}$. For an intuitive understanding, it is enough to observe that any computational element satisfying the restriction of $S P_{1}$ must also satisfy the restriction of $S P_{0}$ since the restriction of $S P_{1}$ is stronger than that of $S P_{0}$. Therefore, every implementation of $S P_{1}$ (ignoring component c) is an implementation of $S P_{0}$.

The form of $S P_{1}$, above, strongly suggests module $m$, below, as a possible implementation for $S P_{1}$ (i.e. $m \in S P_{1}$ ). It is easily shown, by constructive proof, that $m$ is indeed an implementation for $S P_{1}$.

$$
\begin{aligned}
& m=\text { module } \\
& f=\lambda a \in \mathbb{N} . a+27, \\
& c=3 \text {, } \\
& g=\lambda a \in \mathbb{N} \cdot f(a)-c \\
& \text { proof } \\
& \lambda a \in \mathbb{N} .\langle e q,\langle e q, e q\rangle\rangle \\
& \text { end } \in S P_{1}
\end{aligned}
$$

We observe that module $m$ does not quite satisfy of the original specification $S P_{0}$, since $m$ contains the extra component $c$. However, by appealing to Fact 8.1, we can use the abstraction function $h$ and proof map $p$ to get the module

$$
\langle h(c e(m)), p(c e(m))(p f(m))\rangle \in S P_{0}
$$

Here,

$$
\begin{aligned}
& h(c e(m))=\text { module } \\
& \qquad \begin{array}{l}
f=\lambda a \in \mathbb{N} . a+27, \\
g=\lambda a \in \mathbb{N} . f(a)-3 \\
\text { end } \in \operatorname{Sig}\left(S P_{0}\right)
\end{array}
\end{aligned}
$$

and

$$
p(c e(m))(p f(m))=\lambda a \in \mathbb{N} .\langle e q, e q\rangle \in A x\left[S P_{0}\right](h(c e(m)))
$$

Remark In practice, we do not bother calculating the new witness $p(c e(m))(p f(m))$ since, as far as program development is concerned, we are usually only interested in the computational element of an implementation

\subsection{Basic Properties of Refinement}

In general, for two arbitrary specifications $S P_{1}$ and $S P_{2}$, verifying that $S P_{2}$ is a refinement of $S P_{1}$ is difficult. However, since we have a formal definition of refinement, we can give a collection of useful laws that can help make refinements. It is important to be aware that refinement laws must be proved, otherwise there is no guarantee that refinement yields acceptable specifications and implementations. The laws given below are only the most basic laws of refinement, and are in no way a complete 
collection. If a sufficient number of useful laws can be developed, they can be built up into a calculus. Rather than guess a refinement, and then verify it afterwards, the motivation behind a calculus is that we refine a specification by systematically applying the laws of the calculus until we arrive at an acceptable refinement.

The first property we give is that the specification refinement relation is transitive:

Theorem 8.2 (Transitivity of $\complement_{h}$ )

If $S P_{1} \sqsubseteq_{h_{1}} S P_{2}$ and $S P_{2} \sqsubseteq_{h_{2}} S P_{3}$ then $S P_{1} \sqsubseteq_{h_{1} \circ h_{2}} S P_{3}$

\section{Proof Given in Figure $8.2 \square$}

The transitivity property of $\sqsubseteq$ is particularly useful as it allows us to refine a specification via a series of intermediate refinements. For example, we might refine a specification $S P_{0}$ to $S P_{n}$ as follows:

$$
S P_{0} \sqsubseteq_{h_{1}} S P_{1} \sqsubseteq_{h_{2}} S P_{2} \ldots \sqsubseteq_{h_{n-1}} S P_{n-1} \sqsubseteq_{h_{n}} S P_{n}
$$

where $S P_{1}, \ldots, S P_{n-1}$ are intermediate refinements; and $h_{1}, \ldots, h_{n}$ are abstraction maps. Given such a series of refinements, we can use Theorem 8.2 to justify that $S P_{0} \sqsubseteq_{h} S P_{n}$ where $h=h_{1} \circ h_{2} \circ \ldots \circ h_{n}$.

Fact 8.2 If $S P_{1} \sqsubseteq S P_{2}$ and $S P_{2} \sqsubseteq S P_{3}$ then $S P_{1} \sqsubseteq S P_{3}$.

If two specifications are equivalent under specification equality $\Leftrightarrow$-they need not be identical--then they are also refinements of each other:

Theorem 8.3 If $S P_{1} \Leftrightarrow S P_{2}$ then $S P_{1} \sqsubseteq S P_{2}$ and $S P_{2} \sqsubseteq S P_{1}$

Proof If $S P_{1} \Leftrightarrow S P_{2}$ then by the definition of $\Leftrightarrow$ we can deduce that $\operatorname{Sig}\left(S P_{1}\right)=$ $\operatorname{Sig}\left(S P_{1}\right)$ and that the restrictions of $S P_{1}$ and $S P_{2}$ are equivalent:

(1). $\forall x \in \operatorname{Sig}\left(S P_{1}\right) \cdot A x\left(S P_{1}\right)(x) \Leftrightarrow A x\left(S P_{2}\right)(x)$

From (1) we may deduce that there exists two proof maps $p_{1}$ and $p_{2}$ such that,

$p_{1} \in \forall x \in \operatorname{Sig}\left(S P_{2}\right) . A x\left(S P_{2}\right)(x) \Rightarrow A x\left(S P_{1}\right)(x)$

$p_{2} \in \forall x \in \operatorname{Sig}\left(S P_{1}\right) \cdot A x\left(S P_{1}\right)(x) \Rightarrow A x\left(S P_{2}\right)(x)$

By the definition of specification refinement, proof maps $p_{1}$ and $p_{2}$ immediately give us that $S P_{1} \sqsubseteq S P_{2}$ and $S P_{2} \sqsubseteq S P_{1}$.

Theorem 8.3 is useful. It allows us to conclude that if we rearrange the structure of a specification to produce an equivalent specification, then the rearranged specification and the original specification are refinements of each other. Consequently, many of the properties of structured specifications given in Chapter 7 can also be used to help make refinements. For example, we may conclude that $S P \backslash i \backslash j \sqsubseteq S P \backslash(i \cup j)$ and $S P \backslash(i \cup j) \sqsubseteq S P \backslash i \backslash j$ since we have already shown that $S P \backslash i \backslash j \Leftrightarrow S P \backslash(i \cup j)$ in Theorem 7.6. 
Given $S P_{1} \sqsubseteq_{h_{1}} S P_{2}$ and $S P_{2} \sqsubseteq_{h_{2}} S P_{3}$ we can deduce that there exists two proof maps $p_{1}$ and $p_{2}$ such that:

$p_{1} \in \operatorname{Res}\left(S P_{1}, S P_{2}, h_{1}\right)$ where $h_{1} \in \operatorname{Sig}\left(S P_{2}\right) \rightarrow \operatorname{Sig}\left(S P_{1}\right)$

$p_{2} \in \operatorname{Res}\left(S P_{2}, S P_{3}, h_{2}\right)$ where $h_{2} \in \operatorname{Sig}\left(S P_{3}\right) \rightarrow \operatorname{Sig}\left(S P_{2}\right)$

The proof proceeds as follows:

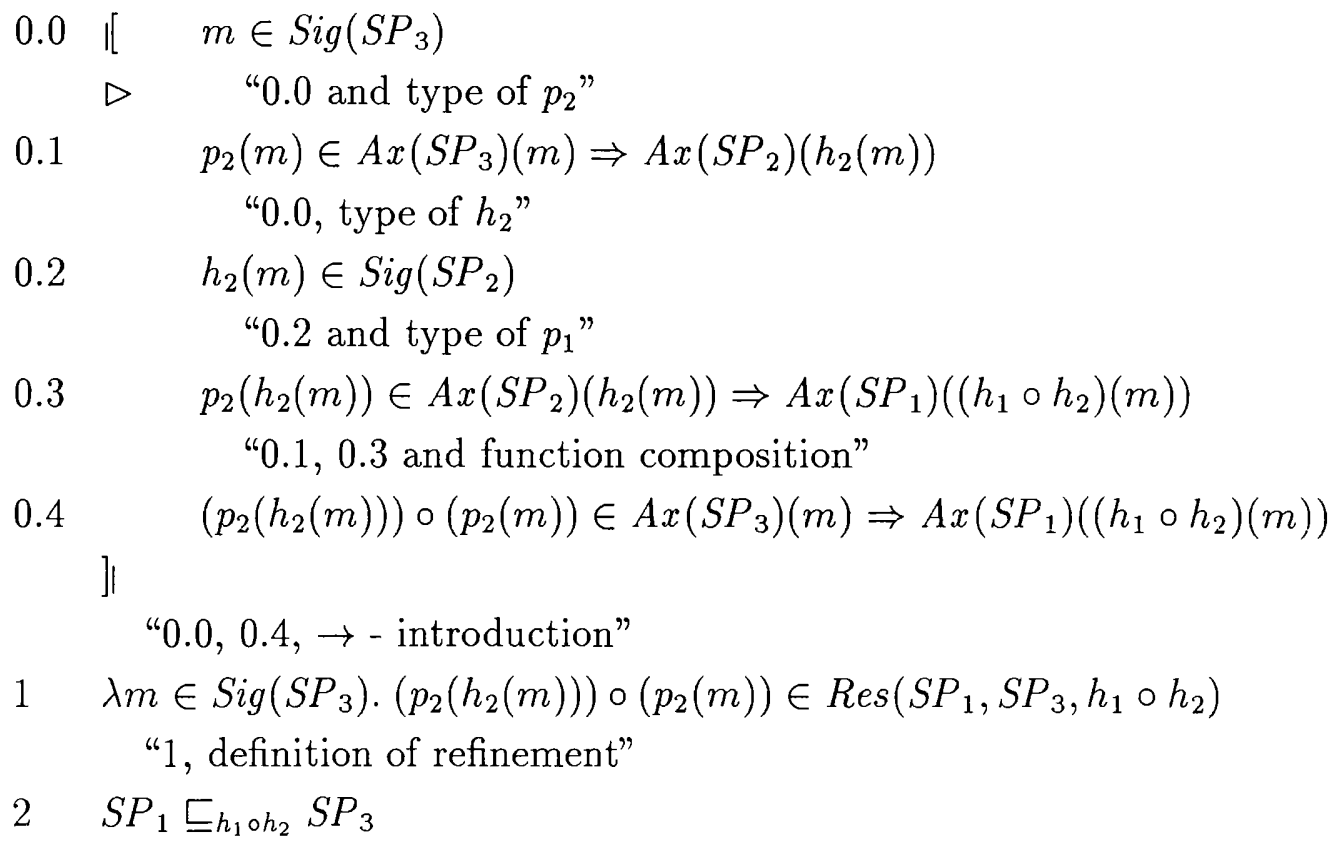

Figure 8.2: A proof of Theorem 8.2

We can also use Theorem 8.3 to conclude that any specification is a refinement of itself. That is to say, the refinement relation is reflexive:

Theorem 8.4 (Reflexivity) $S P \sqsubseteq S P$.

Proof Follows immediately by Theorem 8.3 since $S P=S P$ implies $S P \Leftrightarrow S P \square$

Simple refinement enjoys a kind of anti-symmetry:

Fact 8.3 If $S P_{1} \sqsubseteq S P_{2}$ and $S P_{2} \sqsubseteq S P_{1}$ then $S P_{1} \Leftrightarrow S P_{2}$.

As an aside, general refinement is transitive and reflexive, just like specification refinement:

Fact 8.4 Let $P, Q$, and $R$ be types. If $P \sqsubseteq^{f} Q$ and $Q \sqsubseteq^{g} R$ then $P \sqsubseteq^{f \circ g} R$.

Fact 8.5 Let $I d_{P} \in P \rightarrow P$ be an identity function. $P \check{I d}^{I d_{P}} P \square$

To summarise this section: we have given a collection of laws that allow us to refine specifications via a series of intermediate refinements. 


\subsection{Refinement of Canonical Specifications}

In this section, we consider the refinement of canonical specifications; that is, specifications that do not use structuring operations. Most refinements of canonical specifications fall into three broad categories: strengthening restrictions, adding components to signatures, and decomposing canonical specifications into structured specifications. In the following we give refinement laws for all three categories mentioned above. We will also show that we can refine a specification by refining its signature using general refinement.

\subsubsection{Strengthening Restrictions}

Theorem 8.5, below, says that a specification is refined by strengthening its restriction. Typically, a restriction is strengthened by adding more axioms, and this fact gives us Fact 8.6 below. We can also use the enrich and union operators to add new restrictions to a specification. Consequently, Fact 8.6 justifies the refinement laws given in Facts 8.7 and 8.8 .

Theorem 8.5 If $\forall m \in S . R_{2}(m) \Rightarrow R_{1}(m)$ then $\left\langle S \mid(m) R_{1}\right\rangle \sqsubseteq\left\langle S \mid(m) R_{2}\right\rangle$

Proof Follow immediately from the definition of specification refinement.

Fact 8.6 $\left\langle S \mid(m) R_{1}\right\rangle \sqsubseteq\left\langle S \mid(m) R_{1} \times R_{2}\right\rangle$ where $\llbracket m \in S \triangleright R_{2}(m)$ type $] \square$

Fact 8.7 $S P \sqsubseteq S P \triangleleft \Lambda\left\langle\Phi \mid R_{2}\right\rangle$ where $\llbracket\left[m \in S i g(S P) \triangleright R_{2}(m)\right.$ type $] \square$

Fact 8.8 $S P_{1} \sqsubseteq S P_{1} \cup S P_{2}$ assuming $\operatorname{Sig}\left(S P_{1}\right)=\operatorname{Sig}\left(S P_{2}\right)$

\subsubsection{Refinement by Adding Components}

When making refinements, we often add auxiliary components which only play an intermediate role in helping to make refinements; for example, auxiliary components are often used to simplify the definitions of other components in a specification. It turns out that adding extra components to a specification yields a refinement. We can use signature concatenation to add new components to a specification, and this gives us the following refinement law:

Fact $8.9\left\langle S_{1} \mid(m) R\right\rangle \sqsubseteq \underset{\substack{N_{1} s_{2} \\ \Pi_{1}}}{ }\left\langle S_{1} \oplus S_{2} \mid\left(R \circ \begin{array}{c}s_{1} S_{2} \\ \Pi_{1}\end{array}\right)(m)\right\rangle$ where $S_{1} \vdash S_{2}$ sig $\square$ Recall that the projection function $\underset{\Pi_{1}}{s_{1} s_{2}} \in\left(S_{1} \oplus S_{2}\right) \rightarrow S_{1}$ takes any computational element $m \in S_{1} \oplus S_{2}$ and removes the components of $m$ that are specified in $S_{2}$. Note that we compose $R$ with $\underset{\Pi_{1}}{S_{1} S_{2}}$ to ensure that the restriction of the refinement is dependent on all $m \in S_{1} \oplus S_{2}$ since $R$ is only dependent on all $m \in S_{1}$. 


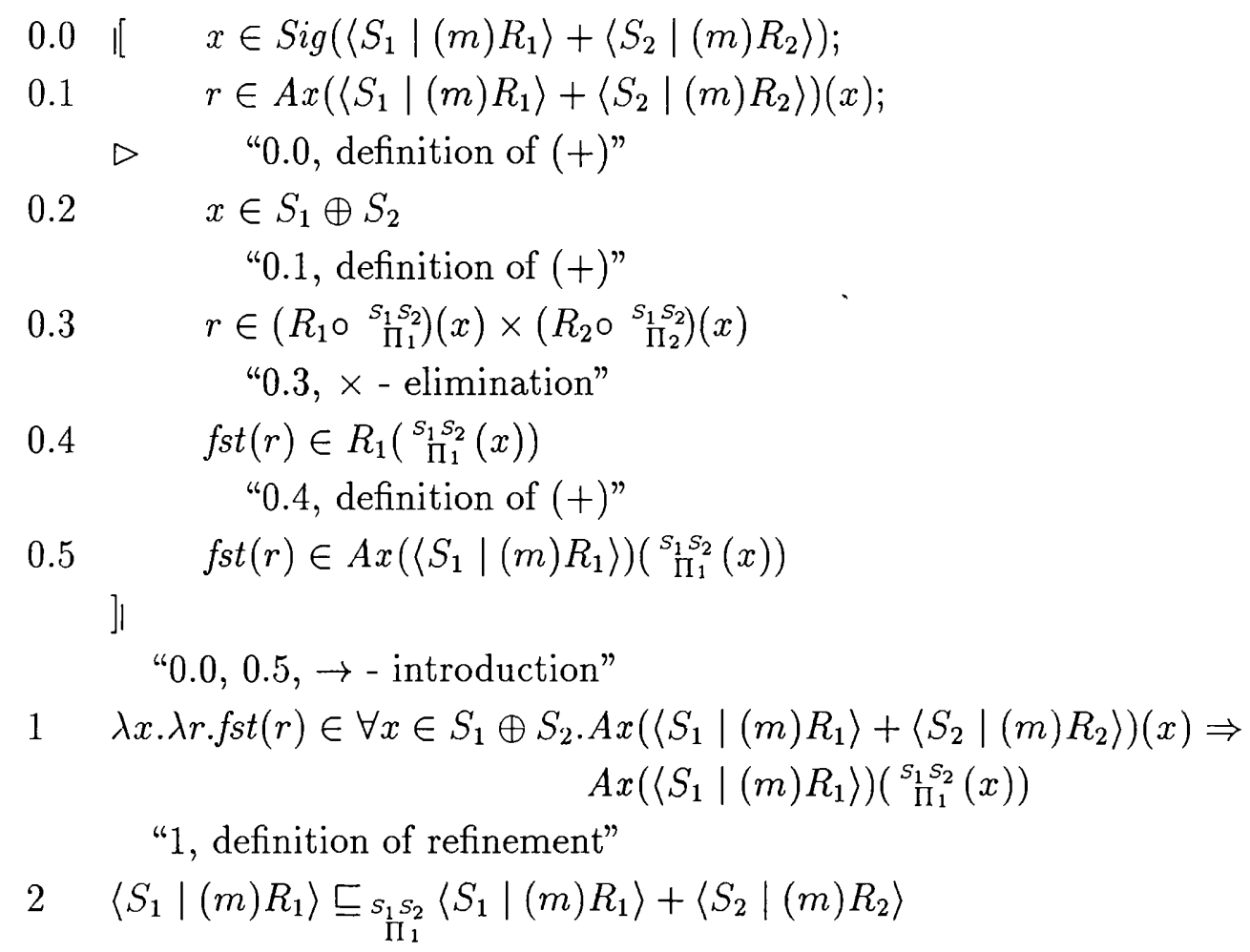

Figure 8.3: Proof of Theorem 8.6

The sum and enrich operators may also be used to add extra components, and restrictions, to a specification. In fact, it can be shown that a specification can be refined by summing it with any specification:

Theorem 8.6 $\left\langle S_{1} \mid(m) R_{1}\right\rangle \sqsubseteq_{\substack{s_{1} s_{2} \\ \Pi_{1}}}\left\langle S_{1} \mid(m) R_{1}\right\rangle+\left\langle S_{2} \mid(m) R_{2}\right\rangle$

Proof Given in Figure 8.3.

Fact $8.10\left\langle S_{1} \mid(m) R_{1}\right\rangle \sqsubseteq{ }_{S_{2} s_{1}}\left\langle S_{2} \mid(m) R_{2}\right\rangle+\left\langle S_{1} \mid(m) R_{1}\right\rangle$

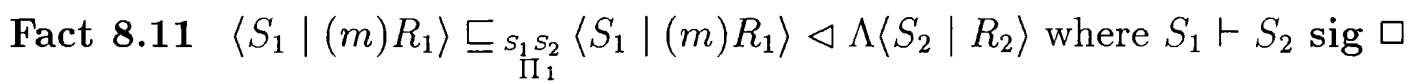

\subsubsection{Refining Signatures}

A signature is a type, and can therefore be refined using general refinement. Moreover, any refinement of the signature of a specification gives a refinement of that specification:

Theorem 8.7 if $S_{1} \sqsubseteq{ }^{f} S_{2}$ then $\left\langle S_{1} \mid(m) R\right\rangle \sqsubseteq f\left\langle S_{2} \mid(m)(R \circ f)(m)\right\rangle$

Proof From the assumption $S_{1} \sqsubseteq^{f} S_{2}$ we may deduce that $f \in S_{2} \rightarrow S_{1}$. By the definition of specification refinement, $\left\langle S_{1} \mid(m) R\right\rangle \sqsubseteq_{f}\left\langle S_{2} \mid(m)(R \circ f)(m)\right\rangle$ holds given any proof map $p \in \forall x \in S_{2} .(R \circ f)(x) \Rightarrow(R \circ f)(x)$. It is easy to verify that $\lambda x \in S_{2} . \lambda r \in(R \circ f)(x) . r$ is a suitable candidate for $p$. 
Note that in Theorem 8.7 the restriction of the refinement is $R \circ f$ : composing $R$ with $f$ ensures that the restriction of the refinement is well typed for all $m \in S_{2}$.

Theorem 8.8, below, says that a signature is refined by adding new components to it. This fact, together with Theorem 8.7 justifies Fact 8.9, given earlier, which says that a specification is refined by adding new components to its signature.

Theorem 8.8 $S_{1} \sqsubseteq \stackrel{S_{1} S_{2}}{\Pi_{1}} S_{1} \oplus S_{2}$ where $S_{1}$ sig and $S_{1} \vdash S_{2}$ sig

Proof $\underset{\Pi_{1}}{s_{1} S_{2}} \in\left(S_{1} \oplus S_{2}\right) \rightarrow S_{1}$

A signature may be refined by refining the types of the components that appear in

it. Such a refinement of the signature of a specification gives a refinement of the specification. For example, the type $U_{1}$ can be refined to $\{P\}_{U_{1}}$ for any $P \in U_{1}$ since $\{P\}_{U_{1}}$ is a subtype of $U_{1}$; formally, $U_{1} \complement^{I d}\{P\}_{U_{1}}$ where $I d \in\{P\}_{U_{1}} \rightarrow U_{1}$ is an identity function. From this fact we get the following refinement law:

Theorem 8.9 $\left\langle y \in U_{1}, S \mid(m) R\right\rangle \sqsubseteq\left\langle y \in\{P\}_{U_{1}}, S \mid(m) R\right\rangle$ for any $P \in U_{1}$

Proof $\quad\left(y \in U_{1}, S\right) \sqsubseteq^{I d}\left(y \in\{P\}_{U_{1}}, S\right)$ where $I d \in\left(y \in\{P\}_{U_{1}}, S\right) \rightarrow\left(y \in U_{1}, S\right)$ is an identity function, and $P \in U_{1}$. The proof then follows by Theorem 8.7

The above refinement law is particularly useful since many specifications contain a type component such as $y \in U_{1}$, and we usually refine it by choosing an actual implementation for $y$ such as $\mathbb{N}, \mathbb{B}$ etc.

The development of refinement laws for types, other than specifications, is beyond the scope of this thesis. Nevertheless, Theorem 8.9 illustrates that, at least in principle, a specification can be refined by refining the types within its signature. It also illustrates the usefulness of having a notion of general refinement that is a generalisation of specification refinement.

\subsubsection{Refinement by Structuring Canonical Specifications}

If a canonical specification contains many components in its signature, or many axioms in its restriction, then it can be difficult to refine. In particular, we can be overwhelmed by the clerical task of managing large numbers of axioms and components. In such cases, refinement can be made easier by decomposing a large canonical specification into smaller, more manageable, pieces. Typically, the decomposition takes the form of expressing the original specification as the composition of several, smaller, specification that are 'glued' together using the specification operators. Once a canonical specification is decomposed into separate specifications, we can refine each specification in isolation. The refinements of each piece of the decomposed specification can then be combined to produce a refinement of the original canonical 
specification. In this section, we consider some laws that allow specifications to be refined by decomposing them using the structuring operators.

Typically, we decompose a canonical specification by splitting its axioms and/or signature into separate pieces. For example, in Chapter 7 we gave Theorem 7.4 that says that a specification can, under certain conditions, be expressed as the sum of, some, two specifications. That sum is a refinement of the original specification:

Theorem 8.10 If $\forall m \in\left(S_{1} \oplus S_{2}\right) . R_{1}(m) \times R_{2}(m) \Leftrightarrow\left(R_{1} \circ \begin{array}{c}S_{1} S_{2} \\ \Pi_{1}\end{array}\right)(m) \times\left(R_{2} \circ \begin{array}{c}S_{1} S_{2} \\ \Pi_{2}\end{array}\right)(m)$ then $\left\langle S_{1} \oplus S_{2} \mid(m) R_{1} \times R_{2}\right\rangle \sqsubseteq\left\langle S_{1} \mid(m) R_{1}\right\rangle+\left\langle S_{2} \mid(m) R_{2}\right\rangle$.

Proof $\left\langle S_{1} \oplus S_{2} \mid(m) R_{1} \times R_{2}\right\rangle \Leftrightarrow\left\langle S_{1} \mid(m) R_{1}\right\rangle+\left\langle S_{2} \mid(m) R_{2}\right\rangle$ by Theorem 7.4. Hence, the proof of the theorem follows immediately by Theorem 8.3.

Fact 8.12 $\left\langle S \mid(m) R_{1} \times R_{2}\right\rangle \sqsubseteq\left\langle S \mid(m) R_{1}\right\rangle \cup\left\langle S \mid(m) R_{2}\right\rangle \square$

Fact 8.12 , above, says that we can refine a specification by using the union operator to split its restriction into separate pieces; this fact follows immediately from the definition of union.

We finish this section by giving some refinement laws, below, that are used to refine canonical specifications to structured specifications. Strictly speaking, the rules given below can also be applied to structured specifications, but since they are mostly used to refine canonical specifications, we give them here. Note that Facts 8.13 and 8.14 use the inverses of the computational element renaming and hiding operators, respectively; these inverse operators were given, previously, in Section 6.11.

Fact 8.13 Let $h={ }_{-}[p \backslash q]_{S i g(S P)}^{-1} \in \operatorname{Sig}(S P)[p \backslash q] \rightarrow \operatorname{Sig}(S P) . S P \sqsubseteq_{h} S P[p \backslash q] \square$

Fact 8.14 Let $h=-\backslash_{S i g(S P)}^{-1} i \in \operatorname{Sig}(S P) \backslash i \rightarrow \operatorname{Sig}(S P) . S P \sqsubseteq_{h} S P \backslash i \square$

Fact 8.15 $S P \sqsubseteq_{f} S P \uparrow f$ where $f \in S \rightarrow \operatorname{Sig}(S P)$ and $S$ sig $\square$

\subsection{Refinement of Structured Specifications}

To be useful, the refinement of specifications must proceed in a stepwise manner. In other words, we want to refine a specification by refining its individual parts in relative isolation, and then combine its refined parts to get a refinement of the original specification. We will give refinement laws that show that if $S P_{1} \sqsubseteq_{h} S P_{2}$ then there exists some abstraction map $g$ such that $F\left(S P_{1}\right) \sqsubseteq_{g} F\left(S P_{2}\right)$ for all specifications $S P_{1}$ and $S P_{2}$, and specification operators $F$. In other words, the structure of a specification carries over to its refinement. 
In practice, most refinements are made using the simple refinement relation (ㄷ) , and we will also show that the specification operators are monotonic with respect to $\sqsubseteq$ :

$$
S P_{1} \sqsubseteq S P_{2} \Rightarrow F\left(S P_{1}\right) \sqsubseteq F\left(S P_{2}\right)
$$

for all specifications $S P_{1}$ and $S P_{2}$, and specification operators $F$. The transitivity of $\sqsubseteq$, and the monotonicity of the specification operators with respect to $\sqsubseteq$, are the two properties that make stepwise refinement an effective notion for structured specifications. The laws given in this section are in no way complete, but do serve to illustrate that structured specifications can be refined in a stepwise manner.

Some of the proofs of the refinement laws presented in this section are rather large, and for presentation, they are given in figures at the end of this chapter.

\subsubsection{Refinement of Rename and Hide}

We begin by considering the refinement of specifications produced using the rename operator. The structure of a specification of the form $S P[p \backslash q]$ carries over to a refinement of such a specification:

\section{Theorem 8.11}

Let $h=\left({ }_{-}[p \backslash q]\right) \circ g \circ\left(-[p \backslash q]_{S i g\left(S P_{2}\right)}^{-1}\right) \in \operatorname{Sig}\left(S P_{2}\right)[p \backslash q] \rightarrow \operatorname{Sig}\left(S P_{1}\right)[p \backslash q]$ where

$$
\begin{array}{lll}
g & \in \operatorname{Sig}\left(S P_{2}\right) \rightarrow \operatorname{Sig}\left(S P_{1}\right) ; \\
-[p \backslash q] & \in \operatorname{Sig}\left(S P_{1}\right) \rightarrow \operatorname{Sig}\left(S P_{1}\right)[p \backslash q] ; \text { and } \\
-[p \backslash q]_{S i g\left(S P_{2}\right)}^{-1} & \in \operatorname{Sig}\left(S P_{2}\right)[p \backslash q] \rightarrow \operatorname{Sig}\left(S P_{2}\right)
\end{array}
$$

If $S P_{1} \sqsubseteq_{g} S P_{2}$ then $S P_{1}[p \backslash q] \sqsubseteq_{h} S P_{2}[p \backslash q]$.

\section{Proof Given in Figure 8.5}

Note that in the above theorem, the abstraction map $h$ is defined using the renaming operator ${ }_{-}[p \backslash q]$, and the inverse renaming operator ${ }_{-}[p \backslash q]_{S i g\left(S P_{2}\right)}^{-1}$. To see how $h$ arises observe that $g$ does not have a suitable type to be an abstraction map between $S P_{1}[p \backslash q]$ and $S P_{2}[p \backslash q]$, but composing $g$ with ${ }_{-}[p \backslash q]$ and ${ }_{-}[p \backslash q]_{S i g\left(S P_{2}\right)}^{-1}$ produces a suitably typed abstraction map $h$.

We may deduce, as a special case of Theorem 8.11, that the rename operator is monotonic with respect to the simple refinement relation $\sqsubseteq$ :

Theorem 8.12 if $S P_{1} \sqsubseteq S P_{2}$ then $S P_{1}[p \backslash q] \sqsubseteq S P_{2}[p \backslash q]$

Proof Let $\operatorname{Sig}\left(S P_{1}\right)=\operatorname{Sig}\left(S P_{2}\right)$ and $g$ be an identity function in Theorem 8.11. Then $h$ is an identity function

We now consider the refinement of specifications that are produced by applying the hide operator. The structure of a specification of the form $S P \backslash i$ carries over to a refinement of such a specification: 


\section{Theorem 8.13}

Let $h=(-\backslash i) \circ g \circ\left(-\backslash_{S i g\left(S P_{2}\right)}^{-1} i\right) \in \operatorname{Sig}\left(S P_{2}\right) \backslash i \rightarrow \operatorname{Sig}\left(S P_{1}\right) \backslash i$ where

$$
\begin{array}{lll}
g & \in \operatorname{Sig}\left(S P_{2}\right) \rightarrow \operatorname{Sig}\left(S P_{1}\right) ; \\
-\backslash i & \in & \operatorname{Sig}\left(S P_{1}\right) \rightarrow \operatorname{Sig}\left(S P_{1}\right) \backslash i \text {; and } \\
-\backslash_{\operatorname{Sig}\left(S P_{2}\right)} i & \in & \operatorname{Sig}\left(S P_{2}\right) \backslash i \rightarrow \operatorname{Sig}\left(S P_{2}\right)
\end{array}
$$

If $S P_{1} \sqsubseteq_{g} S P_{2}$ then $S P_{1} \backslash i \sqsubseteq_{h} S P_{2} \backslash i$.

\section{Proof Similar to that of Theorem 8.11 above $\square$}

Note that in the above theorem, the abstraction map $h$ is defined using the hiding operator $-\backslash i$, and the inverse hiding operator $-\backslash_{S i g\left(S P_{2}\right)}^{-1}$. To see how $h$ arises observe that $g$ does not have a suitable type to be an abstraction map between $S P_{1} \backslash i$ and $S P_{2} \backslash i$, but composing $g$ with $-\backslash i$ and $-_{S i g\left(S P_{2}\right)}^{-1} i$ produces a suitably typed abstraction $\operatorname{map} h$.

We may deduce, as a special case of Theorem 8.13 , that the hide operator is monotonic with respect to the simple refinement relation $\sqsubseteq$ :

Theorem 8.14 if $S P_{1} \sqsubseteq S P_{2}$ then $S P_{1} \backslash i \sqsubseteq S P_{2} \backslash i$

Proof Let $\operatorname{Sig}\left(S P_{1}\right)=\operatorname{Sig}\left(S P_{2}\right)$ and $g$ be an identity function in Theorem 8.13. Then $h$ is an identity function

\subsubsection{Refinement of Union}

Given a specification of the form $S P_{1} \cup S P_{2}$, if we refine $S P_{1}$ and $S P_{2}$ to some specifications $S P_{3}$ and $S P_{4}$, respectively, then the signatures of $S P_{3}$ and $S P_{4}$ must be equal for $S P_{3} \cup S P_{4}$ to be a specification. If $S P_{1}$ and $S P_{2}$ are refined using the same abstraction map then the signatures of $S P_{3}$ and $S P_{4}$ are equal. In that case $S P_{3} \cup S P_{4}$ is a refinement of $S P_{1} \cup S P_{2}$ :

\section{Theorem 8.15}

If $S P_{1} \sqsubseteq_{h} S P_{3}$ and $S P_{2} \sqsubseteq_{h} S P_{4}$ then $S P_{1} \cup S P_{2} \sqsubseteq_{h} S P_{3} \cup S P_{4}$ where $h \in \operatorname{Sig}\left(S P_{3}\right) \rightarrow$ $\operatorname{Sig}\left(S P_{1}\right)$ assuming $\operatorname{Sig}\left(S P_{1}\right)=\operatorname{Sig}\left(S P_{2}\right)$ and $\operatorname{Sig}\left(S P_{3}\right)=\operatorname{Sig}\left(S P_{4}\right)$

Proof The proof is given in Figure 8.6.

As a special case of Theorem 8.15, we may deduce that the union operator is monotonic with respect to the simple refinement relation $\sqsubseteq$ :

Theorem 8.16 if $S P_{1} \sqsubseteq S P_{3}$ and $S P_{2} \sqsubseteq S P_{4}$ then $S P_{1} \cup S P_{2} \sqsubseteq S P_{3} \cup S P_{4}$ assuming that the signatures of $S P_{1}, S P_{2}, S P_{3}$ and $S P_{4}$ are all equal.

Proof Let the signatures of $S P_{1}, S P_{2}, S P_{3}$ and $S P_{4}$ be equal in Theorem 8.15, and let $h$ be an identity function $\square$ 


\subsubsection{Refinement of Sum and Enrich}

We may refine the sum of any two specifications be refining them independently of each other and then summing their refinements:

\section{Theorem 8.17}

Let $S_{i}=\operatorname{Sig}\left(S P_{i}\right)$ for $1 \leq i \leq 4$, and $h=\lambda m . f\left(\underset{\Pi_{1} S_{4}}{\Pi_{1}}(m)\right) \oplus g\left({ }_{\Pi_{2}}^{S_{3} s_{4}}(m)\right) \in\left(S_{3} \oplus S_{4}\right) \rightarrow$ $\left(S_{1} \oplus S_{2}\right)$ where $f \in S_{3} \rightarrow S_{1}$ and $g \in S_{4} \rightarrow S_{2}$. If $S P_{1} \sqsubseteq_{f} S P_{3}$ and $S P_{2} \sqsubseteq_{g} S P_{4}$ then $S P_{1}+S P_{2} \sqsubseteq_{h} S P_{3}+S P_{4}$

\section{Proof The proof is given in Figure 8.7.}

Note that in the above theorem, the abstraction function $h$ is defined using the projection functions $\underset{\Pi_{1}}{s_{3} s_{4}} \in\left(S_{3} \oplus S_{4}\right) \rightarrow S_{3}$ and $\underset{\Pi_{2}}{S_{3} s_{4}} \in\left(S_{3} \oplus S_{4}\right) \rightarrow S_{4}$; and the concatenation operator $\oplus$. To see how $h$ arises observe that $h$ takes any $m \in S_{3} \oplus S_{4}$ and applies $f$ to that part of $m$ specified by $S_{3}$ (i.e. to ${ }_{\Pi_{1}}^{S_{3} s_{4}}(m)$ ); and $h$ applies $g$ to that part of $m$ specified by $S_{4}$ (i.e. to ${ }_{3} s_{4}(m)$ ).

We may deduce, as a special case of Theorem 8.17 , that sum is monotonic with respect to simple refinement:

Theorem 8.18 if $S P_{1} \sqsubseteq S P_{3}$ and $S P_{2} \sqsubseteq S P_{4}$ then $S P_{1}+S P_{2} \sqsubseteq S P_{3}+S P_{4}$

Proof Let $f$ and $g$ be identity functions in Theorem 8.17. Then $h$ is an identity function $\square$

We now consider refining specifications that are produced by the enrich operator. Such specifications have the form $S P \triangleleft \Lambda\langle S \mid R\rangle$, and are refined by refining $S P$ :

\section{Theorem 8.19}

Let $S_{i}=\operatorname{Sig}\left(S P_{i}\right)$ for $1 \leq i \leq 2$, and $h=\lambda m \cdot g\left(\stackrel{S_{2} S}{\Pi_{1}}(m)\right) \oplus \stackrel{S_{2} S}{\Pi_{2}}(m) \in S_{2} \oplus S \rightarrow S_{1} \oplus S$ where $g \in S_{2} \rightarrow S_{1}$ and $S_{2} \vdash S$ sig. If $S P_{1} \sqsubseteq_{g} S P_{2}$ then $S P_{1} \triangleleft \Lambda\langle S \mid R\rangle \sqsubseteq_{h} S P_{2} \triangleleft$ $\Lambda\langle S \mid R \circ h\rangle$.

Proof Similar to that of Theorem 8.17 above.

The following is an explanation of Theorem 8.19 above. The abstraction map $h$ arises for a similar reason as the abstraction map $h$ in Theorem 8.17 (see the discussion after Theorem 8.17). The assumption $S_{2} \vdash S$ sig is necessary to ensure that the refinement is well typed. We also compose $R$ with $h$, in the enrichment of the refinement, to make the refinement well typed: this composition makes the restriction of the enrichment dependent on all $m \in S_{2} \oplus S$.

We may deduce, as a special case of Theorem 8.19, that the enrich operator is monotonic with respect to the simple refinement relation $\sqsubseteq$ : 
Theorem 8.20 if $S P_{1} \sqsubseteq S P_{2}$ then $S P_{1} \triangleleft \Lambda\langle S \mid R\rangle \sqsubseteq S P_{2} \triangleleft \Lambda\langle S \mid R\rangle$

Proof Let $g$ be an identity function in Theorem 8.19. Then $h$ is also an identity function.

Strengthening the restriction of an enrichment also yields a refinement of an enrich operation:

Theorem 8.21

$S P \triangleleft \Lambda\left\langle S \mid R_{1}\right\rangle \sqsubseteq S P \triangleleft \Lambda\left\langle S \mid R_{2}\right\rangle$ if $\forall m \in \operatorname{Sig}(S P) \oplus S . R_{2}(m) \Rightarrow R_{1}(m)$

Proof Apply Theorem $8.5 \square$

\subsubsection{Refinement of Translate and Derive}

Given any specification of the form $S P_{1} \uparrow f$, and any refinement $S P_{2}$ of $S P_{1}$, then $S P_{2} \uparrow f$ is not always a refinement of $S P_{1} \uparrow f$. This is because for $S P_{2} \uparrow f$ to be a valid specification the signature of $S P_{2}$ must be equal to the range of $f$. This requirement is equivalent to saying that the signatures of $S P_{1}$ and $S P_{2}$ must be equal. In that case, we can show that translate is monotonic with respect to $\sqsubseteq$ :

\section{Theorem 8.22}

if $S P_{1} \sqsubseteq S P_{2}$ then $S P_{1} \uparrow f \sqsubseteq S P_{2} \uparrow f$ for any $S$ sig and $f \in S \rightarrow \operatorname{Sig}\left(S P_{2}\right)$

Proof Let $g$ be an identity function in Theorem 8.23 below

\section{Theorem 8.23}

If $S P_{1} \sqsubseteq_{g} S P_{2}$ then $S P_{1} \uparrow(g \circ f) \sqsubseteq S P_{2} \uparrow f$ for any $S$ sig, $f \in S \rightarrow \operatorname{Sig}\left(S P_{2}\right)$ and $g \in \operatorname{Sig}\left(S P_{2}\right) \rightarrow \operatorname{Sig}\left(S P_{1}\right)$

Proof The proof is given in Figure $8.8 \square$

Theorem 8.22 is a special case of Theorem 8.23. Theorem 8.23 has limited applications since it is only applicable to translate expressions whose translate maps have the form $g \circ f$. In practice, most refinements are made using the simple refinement relation $\sqsubseteq$, so that Theorem 8.22 is sufficient to refine most applications of translate.

We now consider refinements involving the derive operator. To begin with, the structure of a specification of the form $S P \downarrow f$ carries over to a refinement of such a specification:

\section{Theorem 8.24}

If $S P_{1} \sqsubseteq g S P_{2}$ then $S P_{1} \downarrow f \sqsubseteq S P_{2} \downarrow(f \circ g)$ for any $S$ sig, $f \in \operatorname{Sig}\left(S P_{1}\right) \rightarrow S$, and $g \in \operatorname{Sig}\left(S P_{2}\right) \rightarrow \operatorname{Sig}\left(S P_{1}\right)$

Proof The proof is given in Figure 8.9. 
Note that in the above theorem we use $f \circ g$ as a derive map for $S P_{2}$. To see how $f \circ g$ arises observe that $f$ is not a suitably typed derive map for $S P_{2}$ - since the domain of $f$ is not equal to $\operatorname{Sig}\left(S P_{2}\right)$-but composing $f$ with $g$ gives a suitably typed derive map for $S P_{2}$.

We can deduce, as a special case of Theorem 8.24, that the derive operator is monotonic with respect to the simple refinement relation $\sqsubseteq$ :

Theorem 8.25 if $S P_{1} \sqsubseteq S P_{2}$ then $S P_{1} \downarrow f \sqsubseteq S P_{2} \downarrow f$ for any $S$ sig and $f \in$ $\operatorname{Sig}\left(S P_{1}\right) \rightarrow S$

Proof Let $\operatorname{Sig}\left(S P_{1}\right)=\operatorname{Sig}\left(S P_{2}\right)$ and $g$ be an identity function in Theorem 8.24

\subsubsection{Summary}

To summarise Section 8.6: the structure of a structured specification carries over to its refinements and, as a special case of this property, the structuring operators are monotonic with respect to the simple refinement relation.

\subsection{An Example Refinement}

We present an example to illustrate the use of the refinement laws given in this chapter. We will refine the specification Mean given, previously, in Figure 3.1; the refinement is based on an example in [36]. Recall that Mean supplies a type, named Data, for samples of naturals. Mean also supplies operations to calculate the sum, size and mean of members of Data, and it supplies an operation to add naturals to members of Data. Mean is a canonical specification, and we shall refine it to a structured specification. Consequently, the example will illustrate the refinement of canonical and structured specifications.

We aim for a refinement of Mean that is defined in terms of the specification PointSpec given, previously, in Figure 4.7. PointSpec specifies a product-like type, named Point, for Cartesian points. We refine Mean by choosing to implement Data by Point, such that Data values are pairs that contain the sum and size of a sample of naturals. Our refinement of Mean is named $\mathrm{Mean}_{3}$, and is given in Figure 8.4. An energetic reader would not find it intellectually taxing to verify that the restriction of $M_{e a n_{3}}$ implies the restriction of Mean, but it would be long and tedious work. In practice, $\mathrm{Mean}_{3}$ is arrived at via a series of intermediate refinements. We give an outline of the refinement of Mean. The refinement is made in several stages, and we give a commentary on the refinement steps as we proceed. 


\subsubsection{The Refinement of Mean}

Stage 1 The first move in the refinement of Mean is to combine PointSpec with Mean, and rename the type Point, in PointSpec, to Pair. The renaming simply gives Point a more meaningful name in the context in which it is used. We let $S_{1}=\operatorname{Sig}($ PointSpec $[$ Point $\backslash$ Pair $])$ and $S_{2}=\operatorname{Sig}($ Mean $)$ :

$$
\begin{gathered}
\text { Mean } \\
\sqsubseteq s_{1} S_{2} \text { "Fact } 8.10 " \\
\Pi_{2} " \\
\quad \text { PointSpec }[\text { Point } \backslash \text { Pair }]+\text { Mean }
\end{gathered}
$$

The above refinement supplies the operations supplied by PointSpec and Mean. We aim to use the components in PointSpec to refine the components and restriction of Mean. We rewrite the above refinement in a form that reveals the operations and restrictions supplied by Mean:

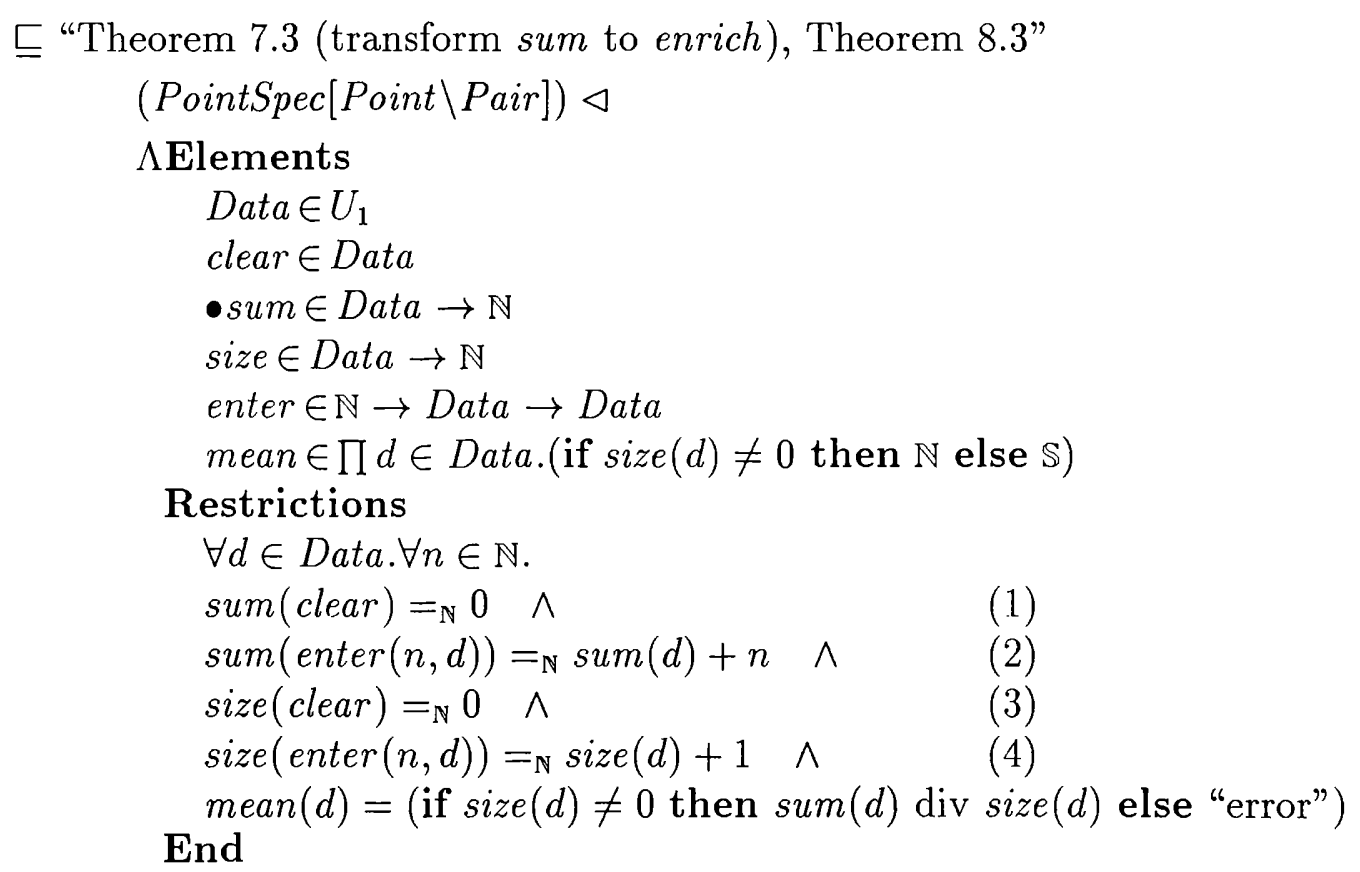

Next, we add our main design decision that Data is to be implemented by Pair. We do this in two refinement steps. Firstly, we refine the type of Data (i.e. $U_{1}$ ) to $\{\text { Pair }\}_{U_{1}}$; such a refinement says that Data equals Pair. Consequently, operations on Pair values are valid on Data values, and vice versa. By Theorem 8.9, replacing the type of Data with $\{\text { Pair }\}_{U_{1}}$ results in a refinement of the above refinement. We call the resultant refinement $\mathrm{Mean}_{2}$, and omit writing it out as it is identical to the above refinement, except that Data $\in U_{1}$ is replaced by Data $\in\{\text { Pair }\}_{U_{1}}$ in Mean ${ }_{2}$.

$$
\text { ᄃ "Theorem 8.9" }
$$

$\mathrm{Mean}_{2}$ 
The second step in implementing Data by Pair is to specify that Data values are pairs that contain the size and sum of a sample of naturals:

巨"Fact 8.7 (any enrichment is a refinement)"

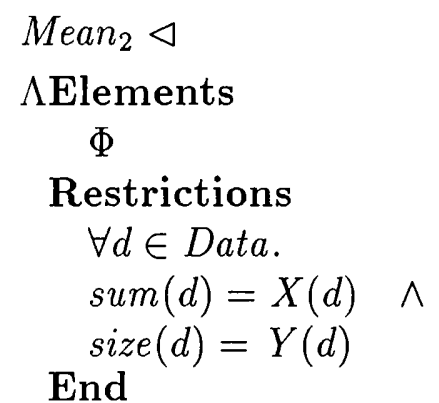

Note that the signature of the above enrichment of $M_{e a n}$ is empty $(\Phi)$. We use axioms (a) and (b) to calculate new restrictions that subsume the old restrictions (1)-(4). We first consider axioms (2) and (4). By substituting $X$ for sum, and $Y$ for size, in the left hand side of (2) and (4), together with straightforward logical manipulation and case analysis, we may calculate that (c), below, implies (2) and (4). Similarly, we may deduce that (d), below, implies (1) and (3).

$$
\begin{array}{lll}
\operatorname{enter}(n, d) & =m k P \operatorname{oint}(\operatorname{sum}(d)+n, \operatorname{size}(d)+1) & (c) \\
\text { clear } & =m k P \operatorname{oint}(0,0) & (d)
\end{array}
$$

Hence, we may show that $(a) \wedge(b) \wedge(c) \wedge(d)$ implies $(1) \wedge(2) \wedge(3) \wedge(4)$. By this fact, and Theorem 8.21, we deduce the following refinement step:

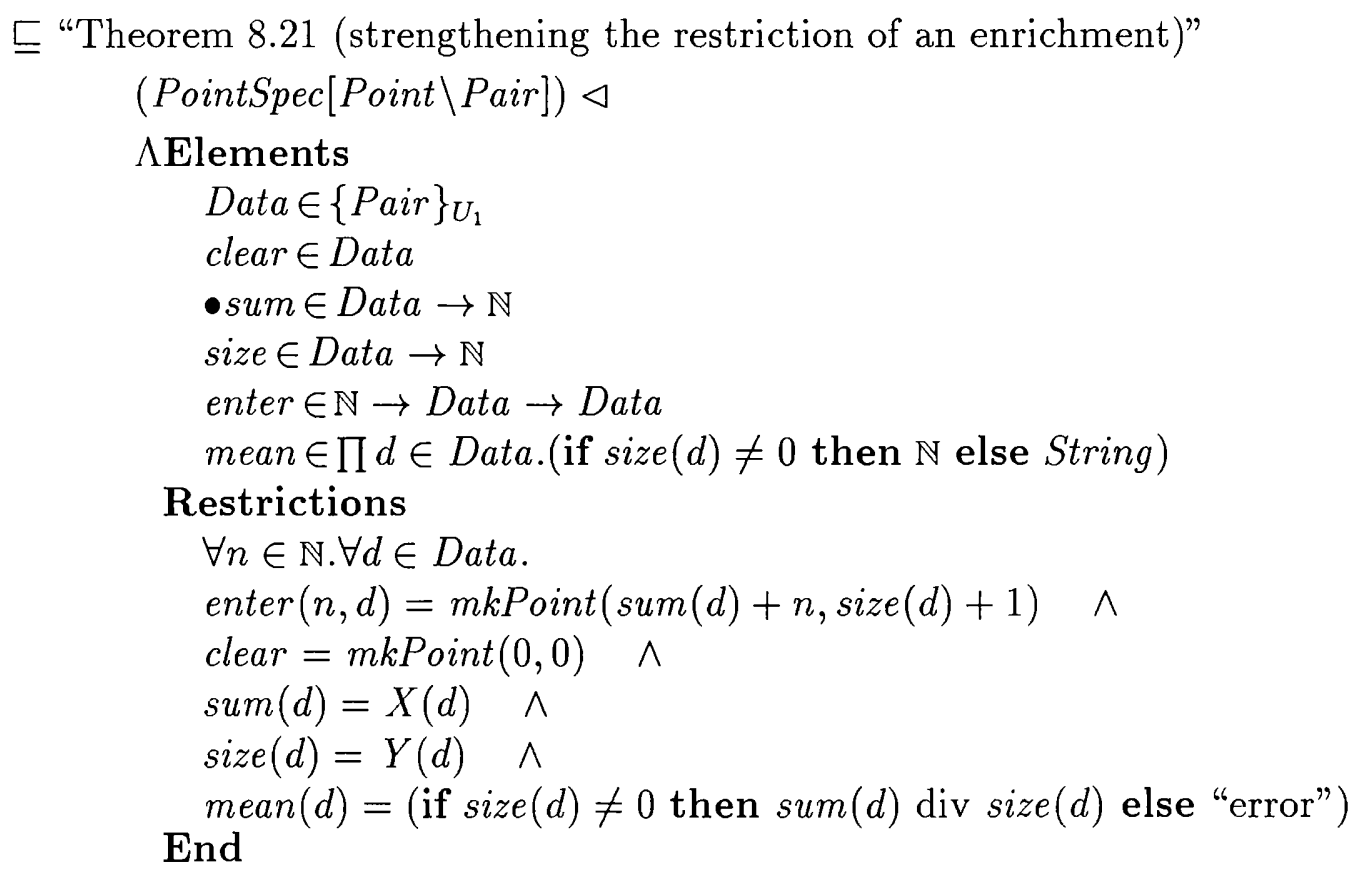

For ease of reference, we give the above enrichment of PointSpec[Point $\backslash$ Pair] the name $\mathrm{Mean}^{+}$:

$=$ "Introduce the name Mean ${ }^{+}$for the above enrichment"

(PointSpec $[$ Point $\backslash$ Pair $]) \triangleleft$ Mean $^{+}$ 
The enrichment $\mathrm{Mean}^{+}$suggests an implementation for Mean based on the operations from PointSpec. Therefore, we shall not refine $\mathrm{Mean}^{+}$any more. We now consider the refinement of (PointSpec[Point $\backslash$ Pair $]$ ).

Stage 2 We will refine (PointSpec[Point $\backslash$ Pair $]$ ) by refining PointSpec. We refine PointSpec by refining the type Point to $\mathbb{N} \times \mathbb{N}$, and strengthening the restriction of PointSpec by specifying the components $X$ and $Y$ to be $f s t$ and snd, respectively:

\section{PointSpec}

$\sqsubseteq$ "Theorem 8.9, Theorem 8.5 (strengthening restrictions)"

\section{Elements}

$$
\begin{aligned}
& \text { Point } \in\{\mathbb{N} \times \mathbb{N}\}_{U_{1},}, \\
& m k \text { Point } \in \mathbb{N} \rightarrow \mathbb{N} \rightarrow \text { Point }, \\
& X \in \text { Point } \rightarrow \mathbb{N}, \\
& Y \in \text { Point } \rightarrow \mathbb{N} \\
& \text { Restrictions } \\
& \forall x, y \in \mathbb{N} . \\
& m k \text { Point }(x, y)=\langle x, y\rangle \wedge \\
& X=f s t \wedge \\
& Y=\text { snd }
\end{aligned}
$$

\section{End}

We give the above refinement of PointSpec the name PointSpec 2 :

$$
\begin{gathered}
=\text { "introducing the name PointSpec } 2 " \\
\text { PointSpec } 2[\text { Point } \backslash \text { Pair }]
\end{gathered}
$$


suggests an implementation based on representing Point values as pairs.

Stage 3 By the refinement step in Stage 2, and the fact that rename is monotonic with respect to $\sqsubseteq$, we can refine (PointSpec[Point $\backslash$ Pair] $)$ as follows:

$$
\begin{aligned}
& \text { PointSpec }[\text { Point } \backslash \text { Pair }] \\
& \sqsubseteq \text { "Stage } 2, \text { Theorem } 8.12 \text { (monotonicity of rename)" } \\
& \text { PointSpec } 2[\text { Point } \backslash \text { Pair }]
\end{aligned}
$$

Stage 4 Now we take the refinement in Stage 1 one step further by replacing PointSpec with PointSpec 2 :

$$
\begin{gathered}
\text { PointSpec }[\text { Point } \backslash \text { Pair }] \triangleleft \text { Mean }^{+} \\
\sqsubseteq \text { "Stage } 3, \text { Theorem } 8.20 \text { (monotonicity of enrich)" } \\
\text { PointSpec }{ }_{2}[\text { Point } \backslash \text { Pair }] \triangleleft \text { Mean }^{+}
\end{gathered}
$$


The above refinement is our final refinement of Mean. All that remains is to combine the refinements steps in Stages 1 and 4 by appealing to the transitivity of specification refinement:

$$
\begin{aligned}
& \text { Mean } \\
& \sqsubseteq s_{s_{1} s_{2}} \text { "Theorem } 8.2 \text { (transitivity of refinement)" } \\
& \Pi_{2} \text { PointSpec } 2[\text { Point } \backslash \text { Pair }] \triangleleft \text { Mean }^{+}
\end{aligned}
$$

In the above refinement step the abstraction map is ${ }_{11}^{s_{1} S_{2}}$ since this is the composition of all the abstraction maps used in the refinement steps in Stages 1 and 4. For ease of reference, we give our final refinement the name $M e a n_{3}$ :

$$
\text { Mean }_{3}=\text { PointSpec }_{2}[\text { Point } \backslash \text { Pair }] \triangleleft \text { Mean }^{+}
$$

A full expansion of $\mathrm{Mean}_{3}$ is given in Figure 8.4.

To summarise this section: we have shown that Mean refines to $M_{e a n_{3}}$ with respect

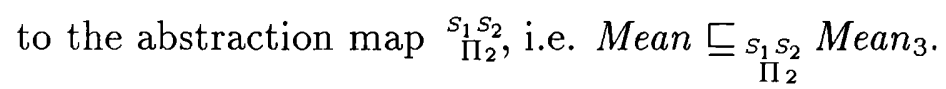

\subsubsection{Discussion of Example}

For our purposes, we assume that $\mathrm{Mean}_{3}$ is the final refinement of Mean-we use Mean $_{3}$ as the starting point for an implementation of Mean in the next chapter. However, some readers may feel unhappy that the final refinement contain some auxiliary components; namely, the components from PointSpec. However, by Fact 8.1, any implementation of $\mathrm{Mean}_{3}$ can be transformed to an implementation of Mean.

The final form of the refinement PointSpec ${ }_{2}[$ Point $\backslash$ Pair $] \triangleleft M e a n^{+}$is not accidental. We deliberately aimed to refine Mean to a structured specification. There are several reason why we should do so. One reason is that this encourages the breaking up of the refinement task into several smaller, and more manageable, refinement tasks that can proceed more or less independently of each other. For example, the refinement of PointSpec in Stage 2 proceeded independently of Stage 1. As we shall see in the next chapter, structured specification are also easier to implement than unstructured specifications-we illustrate this point in the next chapter by implementing $\mathrm{Mean}_{3}$.

Another feature of the refinement of Mean is that it makes use of PointSpec. The reuse of PointSpec illustrates that the reuse of specifications is not limited to making new specifications, but that specifications can also be reused in the refinement process. Reusing specifications in refinements has some useful consequences. For example, we can reuse previous refinements of a specification if it is reused to refine another specification. Furthermore, when we come to implement a refinement, we can reuse implementations of any reused specification. 


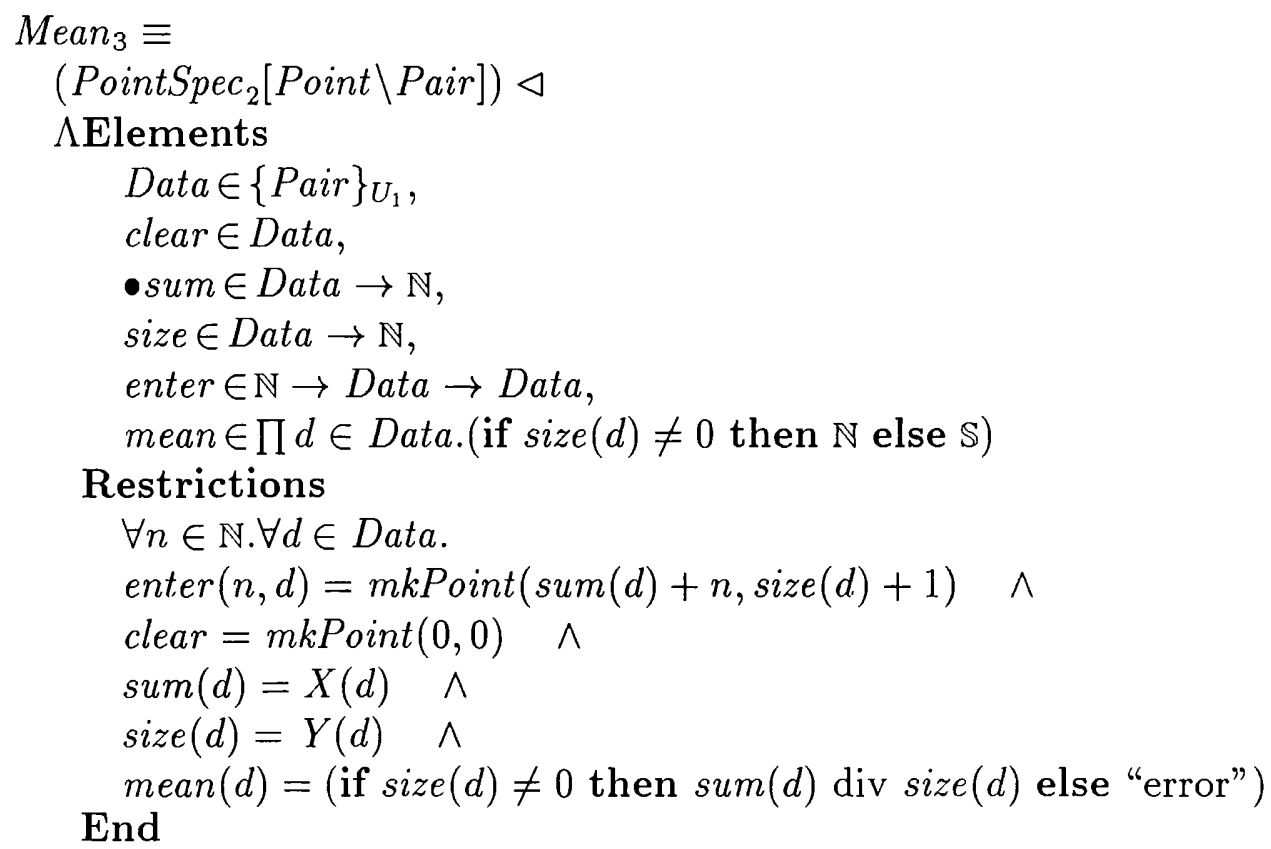

here

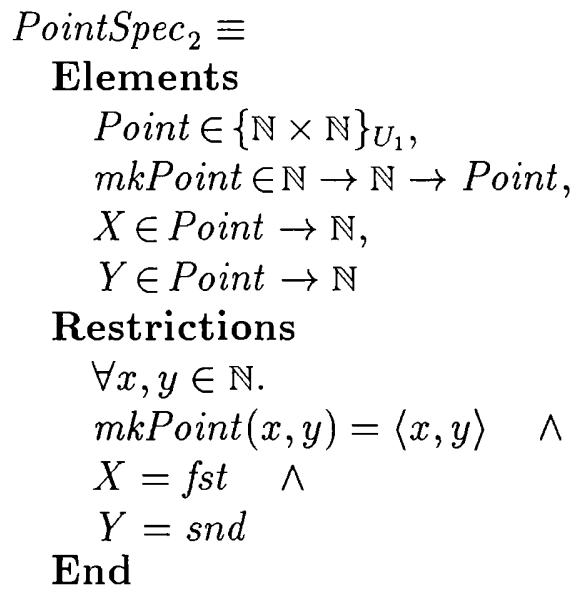

Figure 8.4: A refinement of Mean 


\subsection{Discussion and Summary}

The notion of refinement is well known in many non type-theoretic program development formalisms. Early work on refinement includes [19]. More recently, work has been done on refining algebraic specifications $[50,56]$, and model-oriented specifications in the refinement calculus $[37,38,36]$ and VDM [21]. In such non type-theoretic formalisms, our work on refinement is closely related to the refinement of algebraic specifications in [50]. In [50], the notion of constructors correspond to our abstraction maps. In type theory, our work on refinement is closely related to the work of Burstall et al [6] on deliverables, and Luo's work on specification and refinement in ECC [27]. In particular, [27] gives a definition of refinement, similar to ours, that includes a notion of an abstraction map.

In our experience of refining specifications, we have observed that most refinement steps are made using the simple refinement relation (ㄷ). Abstraction maps are only used for major structural changes to a specification; such as adding new components, or changing the type of a component. In many cases, the modules satisfying a refinement of a specification are not implementations of the original specification; although, by Fact 8.1, an abstraction map can be used to produce an implementation of a specification from an implementation of its refinement. However, in many cases we are happy to accept an implementation of a refinement even if it does not satisfy our original specification. For example, suppose Mean is defined so that Data has the type $\{\operatorname{Set}(\mathbb{N})\}_{U_{1}}$, instead of $U_{1}$, and the axioms of Mean are given in terms of set operators. Furthermore, suppose that we refine Data to $\{\mathbb{N} \times \mathbb{N}\}_{U_{1}}$, and then refine Mean to $\mathrm{Mean}_{3}$. Then an implementation of $\mathrm{Mean}_{3}$ is likely to be more efficient than an implementation of Mean, since an implementation of Mean will use set operations, and these are usually very inefficient. Consequently, we are unlikely to want to transform an implementation of $\mathrm{Mean}_{3}$ to an implementation of Mean.

It might be useful to classify abstraction maps into those that can be ignored, and those that we wish to apply to an implementation of a refinement. By only using particular classes of abstraction maps, we can obtain more specialised definitions of refinement. For example, if we use retract functions as abstraction maps--retract functions throw-away, or permute, components in computational elements-then we get a refinement relation that is equivalent to a Quest [7] style subtype relation.

One of the issues still to be addressed properly is that of managing the refinement task. When we refine a large specification, we can be overwhelmed by the number of steps involved and the clerical task of organising, and keeping track of, these steps. However, type theories promise some solutions to this problem as they lend themselves to the implementation of mechanised proof development systems [8, 23]. In $[8,23]$, it is argued that mechanised systems can assist in choosing appropriate 
refinement steps. If nothing else, mechanisation may help to manage the clerical task of organising the many steps involved in a refinement proof.

Another issue that we have not considered is the development of a refinement calculus for types other than module specifications. Clearly, if we are to refine types in signatures then refinement laws for function types, product types, type universes etc., would be highly desirable. Such types can be refined using general refinement, and a refinement calculus for these types promises to integrate well with specification refinement; such a conclusion is partially justified by our laws for refining signatures. In summary, we have given a definition of refinement for specifications in the framework of Martin-Löf's Type Theory. We have also given a collection of refinement laws that can be used to refine canonical and structured specifications in a piecewise manner. 
$0 \quad$ Let $S_{1}=\operatorname{Sig}\left(S P_{1}\right)$; Let $S_{2}=\operatorname{Sig}\left(S P_{2}\right)$;

$1 \quad g \in S_{2} \rightarrow S_{1}$;

$2 \quad-[p \backslash q] \in S_{1} \rightarrow S_{1}[p \backslash q]$;

$3 \quad-[p \backslash q]_{S_{2}}^{-1} \in S_{2}[p \backslash q] \rightarrow S_{2}$;

$4 \quad$ Let $h=\left(-[p \backslash q] \circ g \circ{ }_{-}[p \backslash q]_{S_{2}}^{-1}\right) \in S_{2}[p \backslash q] \rightarrow S_{1}[p \backslash q]$

"Assumption: $p$ witnesses that $S P_{1} \succeq_{g} S P_{2}$ "

$5 \quad p \in \forall x \in S_{2} . A x\left(S P_{2}\right)(x) \Rightarrow A x\left(S P_{1}\right)(g(x))$

"5, (- $\left.[p \backslash q]_{S_{1}}^{-1} \circ{ }_{-}[p \backslash q]\right)$ is an identity function (Fact 6.35)"

$6 p \in \forall x \in S_{2} . A x\left(S P_{2}\right)(x) \Rightarrow A x\left(S P_{1}\right)\left(\left(-[p \backslash q]_{S_{1}}^{-1} \circ{ }_{-}[p \backslash q] \circ g\right)(x)\right)$

"6, definition of the rename operator"

$7 p \in \forall x \in S_{2} . A x\left(S P_{2}\right)(x) \Rightarrow A x\left(S P_{1}[p \backslash q]\right)((-[p \backslash q] \circ g)(x))$

$8.0 \quad$ ॥ $\quad x \in \operatorname{Sig}\left(S P_{2}[p \backslash q]\right)$

$8.1 .0 \quad \triangleright \quad \| \quad y \in A x\left(S P_{2}[p \backslash q]\right)(x)$

$\triangleright \quad$ "8.1.0, definition of rename"

8.1.1 $y \in A x\left(S P_{2}\right)\left(x[p \backslash q]_{S_{2}}^{-1}\right)$

"8.0, $3, \rightarrow$ - elimination"

8.1.2 $x[p \backslash q]_{S_{2}}^{-1} \in S_{2}$

"8.1.2, 7, $\rightarrow$ - elimination"

8.1 .3

$$
\begin{aligned}
p\left(x[p \backslash q]_{S_{2}}^{-1}\right) \in & A x\left(S P_{2}\right)\left(x[p \backslash q]_{S_{2}}^{-1}\right) \Rightarrow \\
& A x\left(S P_{1}[p \backslash q]\right)\left(\left(-[p \backslash q] \circ g \circ-[p \backslash q]_{S_{2}}^{-1}\right)(x)\right)
\end{aligned}
$$

"8.1.3, definition of $h$ in 4 "

8.1 .4 $p\left(x[p \backslash q]_{S_{2}}^{-1}\right) \in A x\left(S P_{2}\right)\left(x[p \backslash q]_{S_{2}}^{-1}\right) \Rightarrow A x\left(S P_{1}[p \backslash q]\right)(h(x))$

"8.1.4, 8.1.1, $\rightarrow$ - elimination"

8.1 .5

$$
\left(p\left(x[p \backslash q]_{S_{2}}^{-1}\right)\right)(y) \in A x\left(S P_{1}[p \backslash q]\right)(h(x))
$$

]1

]

"8.0, 8.1.0, 8.1.5, $\rightarrow$ - introduction twice"

$9 \quad \lambda x \cdot \lambda y \cdot\left(p\left(x[p \backslash q]_{S_{2}}^{-1}\right)\right)(y) \in \forall x \in S_{2}[p \backslash q] . A x\left(S P_{2}[p \backslash q]\right)(x) \Rightarrow$ $A x\left(S P_{1}[p \backslash q]\right)(h(x))$

" 9 , definition of specification refinement"

10 $S P_{1}[p \backslash q] \sqsubseteq_{h} S P_{2}[p \backslash q]$

Figure 8.5: Proof of Theorem 8.11 
$0 \quad h \in \operatorname{Sig}\left(S P_{3}\right) \rightarrow \operatorname{Sig}\left(S P_{1}\right)$;

"assumption"

$1 \quad \operatorname{Sig}\left(S P_{1}\right)=\operatorname{Sig}\left(S P_{2}\right) ; \operatorname{Sig}\left(S P_{3}\right)=\operatorname{Sig}\left(S P_{4}\right) ;$

"assumption: $p$ witnesses that $S P_{1} \sqsubseteq_{h} S P_{3}$ "

$2 p \in \forall x \in \operatorname{Sig}\left(S P_{3}\right) . A x\left(S P_{3}\right)(x) \Rightarrow A x\left(S P_{1}\right)(h(x))$;

"assumption: $q$ witnesses that $S P_{2} \sqsubseteq_{h} S P_{4}$ "

$3 q \in \forall x \in \operatorname{Sig}\left(S P_{4}\right) . A x\left(S P_{4}\right)(x) \Rightarrow A x\left(S P_{2}\right)(h(x))$

4.0 I $\quad x \in \operatorname{Sig}\left(S P_{3}\right)$

$4.1 .0 \quad \triangleright \quad \mathbb{} \quad y \in A x\left(S P_{3} \cup S P_{4}\right)(x)$

$\triangleright \quad$ "4.1.0, definition of union"

4.1.1 $y \in A x\left(S P_{3}\right)(x) \times A x\left(S P_{4}\right)(x)$

"4.1.1, $\times$ - elimination"

4.1 .2

$f s t(y) \in A x\left(S P_{3}\right)(x)$

"2, 4.0, 4.1.2, $\rightarrow$ - elimination twice"

4.1.3

$(p(x))(f s t(y)) \in A x\left(S P_{1}\right)(h(x))$

"4.1.1, , $\times$ - elimination"

4.1 .4

$\operatorname{snd}(y) \in A x\left(S P_{4}\right)(x)$

"3, 4.0, 4.1.4, $\rightarrow$ - elimination, $x \in \operatorname{Sig}\left(S P_{3}\right)=\operatorname{Sig}\left(S P_{4}\right)$ "

4.1 .5 $(q(x))(\operatorname{snd}(y)) \in A x\left(S P_{2}\right)(h(x))$

"4.1.3, 4.1.5, $\times$ - introduction"

4.1.6

$\langle(p(x))(f s t(y)),(q(x))(\operatorname{snd}(y))\rangle \in A x\left(S P_{1}\right)(h(x)) \times$

$$
A x\left(S P_{2}\right)(h(x))
$$

"4.1.6, definition of union"

$\langle(p(x))(f s t(y)),(q(x))(\operatorname{snd}(y))\rangle \in A x\left(S P_{1} \cup S P_{2}\right)(h(x))$

]1

]

"4.0, 4.1.0, 4.1.7, $\rightarrow$ - introduction twice"

$5 \lambda \lambda x . \lambda y \cdot\langle(p(x))(f s t(y)),(q(x))(\operatorname{snd}(y))\rangle \in \forall x \in \operatorname{Sig}\left(S P_{3}\right)$.

$$
\begin{aligned}
& A x\left(S P_{3} \cup S P_{4}\right)(x) \Rightarrow \\
& A x\left(S P_{1} \cup S P_{2}\right)(h(x))
\end{aligned}
$$

"5, definition of specification refinement"

$6 \quad S P_{1} \cup S P_{2} \sqsubseteq_{h} S P_{3} \cup S P_{4}$

Figure 8.6: Proof of Theorem 8.15 
Let $S_{i}=\operatorname{Sig}\left(S P_{i}\right)$ for $1 \leq i \leq 4$, and $\left.h=\lambda m . f\left(\underset{\Pi_{1} S_{4}}{S_{1} S_{4}}\right)\right) \oplus g\left(\stackrel{{ }_{3} S_{4}}{\Pi_{2}}(m)\right) \in\left(S_{3} \oplus S_{4}\right) \rightarrow$ $\left(S_{1} \oplus S_{2}\right)$ where $f \in S_{3} \rightarrow S_{1}$ and $g \in S_{4} \rightarrow S_{2}$. We are given

1. $S P_{1} \sqsubseteq f \quad S P_{3}$

2. $\mathrm{SP}_{2} \sqsubseteq_{g} \mathrm{SP}_{4}$

We shall require the following facts:

3. $S P_{1} \uparrow S_{1} S_{2} \quad \bigsqcup_{h} \quad\left(S P_{1} \uparrow s_{1} S_{2}\right) \uparrow h$

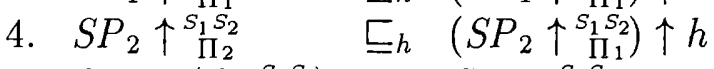

5. $S P_{1} \uparrow\left(f \circ \mathrm{S}_{3}{\Pi_{4}}_{1}\right) \sqsubseteq \quad S P_{3} \uparrow S_{\Pi_{1} S_{4}}$

6. $S P_{2} \uparrow\left(g \circ \begin{array}{c}S_{3} S_{4} \\ \Pi_{2}\end{array}\right) \sqsubseteq S P_{4} \uparrow{\stackrel{S_{3} S_{4}}{\Pi_{2}}}^{2}$

Facts (3) and (4) follow immediately by Theorem 8.15 ; fact (5) follows by Theorem 8.23 and assumption (1); and fact (6) follows by Theorem 8.23 and assumption (2). The proof now proceeds as follows:

$$
S P_{1}+S P_{2}
$$

$=$ "Theorem 7.1 (sum expressed in terms of translate and union)"

$$
\left(S P_{1} \uparrow \begin{array}{c}
s_{1} S_{2} \\
\Pi_{1}
\end{array}\right) \cup\left(S P_{2} \uparrow \begin{array}{c}
s_{1} S_{2} \\
\Pi_{2}
\end{array}\right)
$$

$\complement_{h}$ "(3),(4) and Theorem 8.15 (refinement of union)"

$$
\left(\left(S P_{1} \uparrow \begin{array}{c}
s_{1} s_{2} \\
\Pi_{1}
\end{array}\right) \uparrow h\right) \cup\left(\left(S P_{2} \uparrow \begin{array}{c}
s_{1} s_{2} \\
\Pi_{2}
\end{array}\right) \uparrow h\right)
$$

$=$ "Theorem 7.22 (translate composition)"

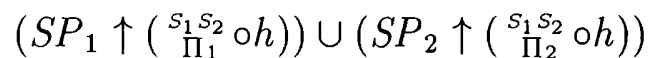

$$
\begin{aligned}
& ="\left(\begin{array}{cc}
s_{1} s_{2} \\
\Pi_{1} \circ h
\end{array}\right)=\left(\begin{array}{ll}
f \circ & s_{3} s_{4} \\
\Pi_{1}
\end{array}\right) \text { and }\left(\begin{array}{cc}
s_{1} s_{2} \\
\Pi_{2} \circ h
\end{array}\right)=\left(\begin{array}{ll}
g \circ s_{3} s_{4} \\
\Pi_{2}
\end{array}\right) "
\end{aligned}
$$

$$
\left(S P_{1} \uparrow\left(f \circ \begin{array}{c}
S_{3} S_{4} \\
\Pi_{1}
\end{array}\right)\right) \cup\left(S P_{2} \uparrow\left(g \circ \begin{array}{cc}
S_{3} S_{4} \\
\Pi_{2}
\end{array}\right)\right)
$$

$\sqsubseteq$ "(5), (6), monotonicity of union w.r.t $\sqsubseteq ”$

$$
\left(S P_{3} \uparrow \begin{array}{c}
s_{3} s_{4} \\
\Pi_{1}
\end{array}\right) \cup\left(S P_{4} \uparrow{ }_{3}^{s_{3} s_{4}} \Pi_{2}\right)
$$

= "Theorem 7.1 (sum expressed in terms of translate and union)"

$$
\mathrm{SP}_{3}+\mathrm{SP}_{4}
$$

By the transitivity of specification refinement, we compose the abstraction maps used in each step of the proof-recalling that $\sqsubseteq=\sqsubseteq_{I d}$-to get $S P_{1}+S P_{2} \sqsubseteq_{h} S P_{3}+S P_{4}$.

Figure 8.7: Proof of Theorem 8.17 
$0 \quad S \operatorname{sig} ; f \in S \rightarrow \operatorname{Sig}\left(S P_{2}\right) ; g \in \operatorname{Sig}\left(S P_{2}\right) \rightarrow \operatorname{Sig}\left(S P_{1}\right) ;$

"assumption: $p$ witnesses that $S P_{1} \sqsubseteq_{g} S P_{2}$ "

$1 p \in \forall x \in \operatorname{Sig}\left(S P_{2}\right) \cdot A x\left(S P_{2}\right)(x) \Rightarrow A x\left(S P_{1}\right)(g(x))$

2.0 [ $\quad x \in S$

$2.1 .0 \triangleright \mathbb{\|} \quad y \in A x\left(S P_{2} \uparrow f\right)(x)$

$\triangleright \quad$ "2.1.0, definition of translate"

2.1.1 $y \in\left(A x\left(S P_{2}\right)\right)(f(x))$

"0, 2.0, $\rightarrow$ - elimination"

2.1.2 $f(x) \in \operatorname{Sig}\left(S P_{2}\right)$

"1, 2.1.1, 2.1.2, $\rightarrow$ - elimination twice"

2.1 .3 $(p(f(x)))(y) \in A x\left(S P_{1}\right)(g(f(x)))$

"2.1.3, definition of translate"

2.1 .4 $(p(f(x)))(y) \in A x\left(S P_{1} \uparrow(g \circ f)\right)(x)$

]

]।

"2.0, 2.1.0, 2.1.4, $\rightarrow$ - introduction twice"

$3 \quad \lambda x . \lambda y \cdot\left(p(f(x))(y) \in \forall x \in S . A x\left(S P_{2} \uparrow f\right)(x) \Rightarrow\right.$ $A x\left(S P_{1} \uparrow(g \circ f)\right)(x)$

" 3 , definition of specification refinement"

$4 \quad S P_{1} \uparrow(g \circ f) \sqsubseteq S P_{2} \uparrow f$

Figure 8.8: Proof of Theorem 8.23 
$0 \quad S$ sig; $f \in \operatorname{Sig}\left(S P_{2}\right) \rightarrow S ; g \in \operatorname{Sig}\left(S P_{2}\right) \rightarrow \operatorname{Sig}\left(S P_{1}\right) ;$

"assumption: $p$ witnesses that $S P_{1} \sqsubseteq_{g} S P_{2}$ "

$1 p \in \forall x \in \operatorname{Sig}\left(S P_{2}\right) \cdot A x\left(S P_{2}\right)(x) \Rightarrow A x\left(S P_{1}\right)(g(x))$;

$2.0 \quad$ ॥ $\quad x \in S$

2.1.0 $\triangleright \quad \mathbb{\|} \quad y \in A x\left(S P_{2} \downarrow(f \circ g)\right)(x)$

$\triangleright \quad$ "2.1.0, definition of derive"

2.1 .1

$y \in \exists z \in S P_{2} \cdot\left[f(g(c e(z))){ }_{S} x\right]$

"2.1.1, $\times$ - elimination"

2.1 .2

$f s t(y) \in S P_{2}$

"2.1.2, x - elimination"

2.1 .3

$c e(f s t(y)) \in \operatorname{Sig}\left(S P_{2}\right)$

"2.1.3, $0, \rightarrow$ - elimination"

2.1 .4

$g(\operatorname{ce}(f s t(y))) \in \operatorname{Sig}\left(S P_{1}\right)$

"2.1.2, $\times$ - elimination"

2.1.5

$p f\left(f_{s t}(y)\right) \in A x\left(S P_{2}\right)(c e(f s t(y)))$

"1, 2.1.3, 2.1.5, $\rightarrow$ - elimination twice"

2.1 .6

$(p(c e(f s t(y))))\left(p f\left(f_{s t}(y)\right)\right) \in A x\left(S P_{1}\right)\left(g\left(c e\left(f_{s t}(y)\right)\right)\right)$

"2.1.4, 2.1.6, $\times$ - introduction, introduce name $m$ "

2.1.7

Let $m=\langle g(c e(f s t(y))),(p(c e(f s t(y))))(p f(f s t(y)))\rangle \in S P_{1}$

"2.1.1, $\times$ - elimination, $E q$ - elimination"

2.1 .8

$\operatorname{snd}(y)=e q \in\left[f(g(\operatorname{ce}(f s t(y))))={ }_{S} x\right]$

"2.1.7, $\times$ - elimination"

2.1 .9

$c e(m)=g\left(c e\left(f_{s t}(y)\right)\right) \in \operatorname{Sig}\left(S P_{1}\right)$

"2.1.8, 2.1.9, substitution"

2.1 .10

$\operatorname{snd}(y)=e q \in\left[f(c e(m))={ }_{S} x\right]$

"2.1.7, 2.1.10, $\times$ - introduction"

2.1 .11

$\langle m, e q\rangle \in \exists z \in S P_{1} \cdot\left[f(c e(z))=_{S} x\right]$

"2.1.11, definition of derive"

2.1 .12

$\langle m, e q\rangle \in A x\left(S P_{1} \downarrow f\right)(x)$

]

]।

"2.0,2.1.0, 2.1.12, $\rightarrow$ - introduction twice"

3

$\lambda x . \lambda y .\langle m, e q\rangle \in \forall x \in S . A x\left(S P_{2} \downarrow(f \circ g)\right)(x) \Rightarrow A x\left(S P_{1} \downarrow f\right)(x)$

"3, definition of specification refinement"

4

$S P_{1} \downarrow f \sqsubseteq S P_{2} \downarrow(f \circ g)$

Figure 8.9: Proof of Theorem 8.24 


\section{Chapter 9}

\section{Implementation}

\subsection{Introduction}

In our type-theoretic specification framework, the development of implementations from specifications is a two step process. First, we refine a specification and then we implement the refined specification. Implementation is the task of finding a module that satisfies a specification. In other words, implementation is the task of constructing a member of the type a specification denotes. To be useful, implementation must proceed in a piecewise manner. That is to say, we want to implement a specification by implementing its constituent pieces, so that the implemented pieces may be glued together to form an implementation of the specification. The "glue" is a collection of module operators that we call implementors. In this chapter, we define several implementors, and use them to give some implementation laws that aid the piecewise implementation of specifications.

Each implementor corresponds to a specification operator, and is used to implement specifications made using its corresponding specification operator. For example, the sum implementor $(+)$ corresponds to the sum specification operator; note that we overload + , so that it denotes both the sum specification operator and the sum implementor. Given any two specifications $S P_{1}$ and $S P_{2}$, and any two modules $m_{1} \in$ $S P_{1}$ and $m_{2} \in S P_{2}$, then $m_{1}+m_{2} \in S P_{1}+S P_{2}$. Some specification operators, such as Union, do not have corresponding implementors, but specifications that use such specification operators can, under certain conditions, be implemented in a piecewise manner.

There are several advantages to the piecewise implementation of specifications. Firstly, we avoid the need to rearrange a specification prior to implementation. Secondly, it puts a tight upper-bound on the amount of recoding we have to do if we change part of a specification. Thirdly, it is more likely that we can reuse imple- 
mentations if a specification is used more than once. This last point is important as it encourages us to make libraries of useful specifications, and their implementations, that can be used in other applications. The piecewise implementation of specifications may not always yield an efficient implementation. However, it is a systematic approach to implementing large specifications, and helps to decompose the implementation task into a number of smaller, more manageable, tasks.

In the following, we ignore the implementation of canonical specifications. We assume that specifications have already been refined prior to implementation, so that the restrictions of canonical specifications suggest possible implementations. Therefore, we assume that canonical specifications can be implemented by a simple constructive proof. Alternatively, if we have a large canonical specification it may first be structured and then implemented using the techniques described in this chapter. Wherever we require the implementation of a canonical specification, we simply state the implementation, although the reader should be aware that work needs to be done to produce such implementations.

\section{$9.2 \quad$ Implementors}

In this section, we give a formal definition of implementors. Implementors are module operators that correspond to some specification operators. The implementors we define correspond to the specification operators rename, hide, sum and derive. Implementors are used to implement specifications of the form $F(S P)$ in a piecewise manner, where $F$ is any one of the specification operators mentioned above, and $S P$ any specification. That is to say, $F(S P)$ may be implemented by replacing $S P$ with any implementation $m \in S P$, and replacing $F$ by its corresponding implementor $f$ :

$$
m \in S P \Rightarrow f(m) \in F(S P)
$$

We show that the piecewise implementation property, described above, follows immediately from the definition of each of the implementors we define.

To avoid the proliferation of operator symbols, we will overload the operator symbols for the specification operators rename, hide, sum and derive, and use them to denote their corresponding implementors.

\subsubsection{The Rename and Hide Implementors}

An application of the rename implementor has the form $m[p \backslash q]$ for $m$ any module, and $p$ and $q$ any component names. $m[p \backslash q]$ is module $m$ with component $p$ renamed to $q$. An application of the hide implementor has the form $m \backslash i$ for $m$ any module, 
and $i$ a set of component names. $m \backslash i$ is module $m$ with all components named in $i$ made local in $m$. The formal definitions of the rename and hide implementors are given below with an explanation following the definitions.

\section{Definition 9.1 (The Rename Implementor)}

Let $S P$ be any specification, and $p, q \in \mathbb{S}$. Given any $m \in S P$, the rename implementor $-[p \backslash q] \in S P \rightarrow S P[p \backslash q]$ is defined as follows:

$$
m[p \backslash q]=\langle c e(m)[p \backslash q], p f(m)\rangle \in S P[p \backslash q]
$$

\section{Definition 9.2 (The Hide Implementor)}

Let $S P$ be any specification, and $i \in \operatorname{Set}(\mathbb{S})$. Given any $m \in S P$, the hide implementor $-\backslash i \in S P \rightarrow S P \backslash i$ is defined as follows:

$$
m \backslash i=\langle c e(m) \backslash i, p f(m)\rangle \in S P \backslash i
$$

Let us consider the definition of the rename implementor. The expression $c e(m)[p \backslash q]$ is the computational element of module $m[p \backslash q]$, and expresses the application of the computational element renaming operator to the computational element of $m$. The witness of $m[p \backslash q]$ is $p f(m)$, which is just the witness of $m$.

From the definition of the rename implementor, we may deduce that a specification of the form $S P[p \backslash q]$ may be implemented in a piecewise manner: if $m \in S P$ then $m[p \backslash q] \in S P[p \backslash q]$. A proof of this property is given in Figure 9.1.

The hide implementor is defined using the computational element hide operator, and its definition is similar, in style, to that of the rename implementor. From the definition of the hide implementor, we may deduce that specifications of the form $S P \backslash i$ may be implemented in a piecewise manner: if $m \in S P$ then $m \backslash i \in S P \backslash i$. The proof of this property is similar to the proof in Figure 9.1.

\subsubsection{The Sum Implementor}

An application of the sum implementor has the form $m_{1}+m_{2}$ where $m_{1}$ and $m_{2}$ are any modules. The module $m_{1}+m_{2}$ supplies all the components in $m_{1}$ and $m_{2}$. A formal definition of the sum implementor is given below, with an explanation following the definition.

\section{Definition 9.3 (Sum Implementation)}

Let $S P_{1}$ and $S P_{2}$ be any specifications. Given any $m_{1} \in S P_{1}$ and $m_{2} \in S P_{2}$, the sum implementor _ + $\in S P_{1} \rightarrow S P_{2} \rightarrow S P_{1}+S P_{2}$ is defined as follows:

$$
m_{1}+m_{2}=\left\langle c e\left(m_{1}\right) \oplus c e\left(m_{2}\right),\left\langle p f\left(m_{1}\right), p f\left(m_{2}\right)\right\rangle\right\rangle \in S P_{1}+S P_{2}
$$




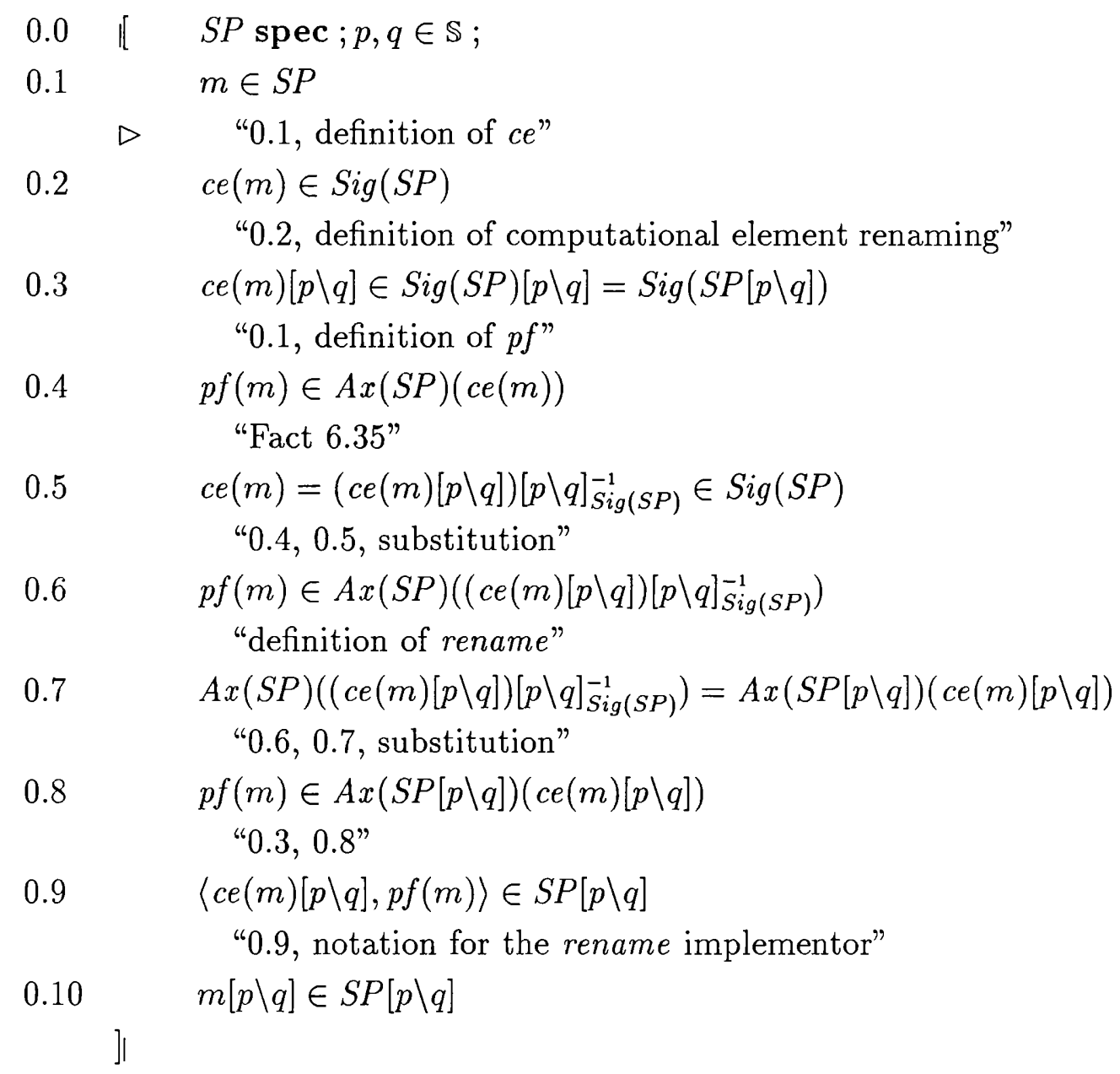

Figure 9.1: A Proof of the Type Correctness of the Rename Implementor 




Figure 9.2: A Proof of the Type Correctness of the Sum Implementor

The sum implementor is defined using the computational element concatenation operator $(\oplus)$. The expression $c e\left(m_{1}\right) \oplus c e\left(m_{2}\right)$ is the computational element of $m_{1}+m_{2}$, and satisfies the signature of $S P_{1}+S P_{2}$. The expression $\left\langle p f\left(m_{1}\right), p f\left(m_{2}\right)\right\rangle$ is the witness of $m_{1}+m_{2}$, and satisfies the restriction of $S P_{1}+S P_{2}$ which is the product of the restrictions of $S P_{1}$ and $S P_{2}$.

From the definition of the sum implementor we get that specifications of the form $S P_{1}+S P_{2}$ may be implemented in a piecewise manner: given any $m_{1} \in S P_{1}$ and $m_{2} \in S P_{2}$ then $m_{1}+m_{2} \in S P_{1}+S P_{2}$. In other words, $S P_{1}+S P_{2}$ is implemented by implementing $S P_{1}$ and $S P_{2}$ independently of each other, and then combining their implementations using the sum implementor. A proof of this property is given in Figure 9.2. 


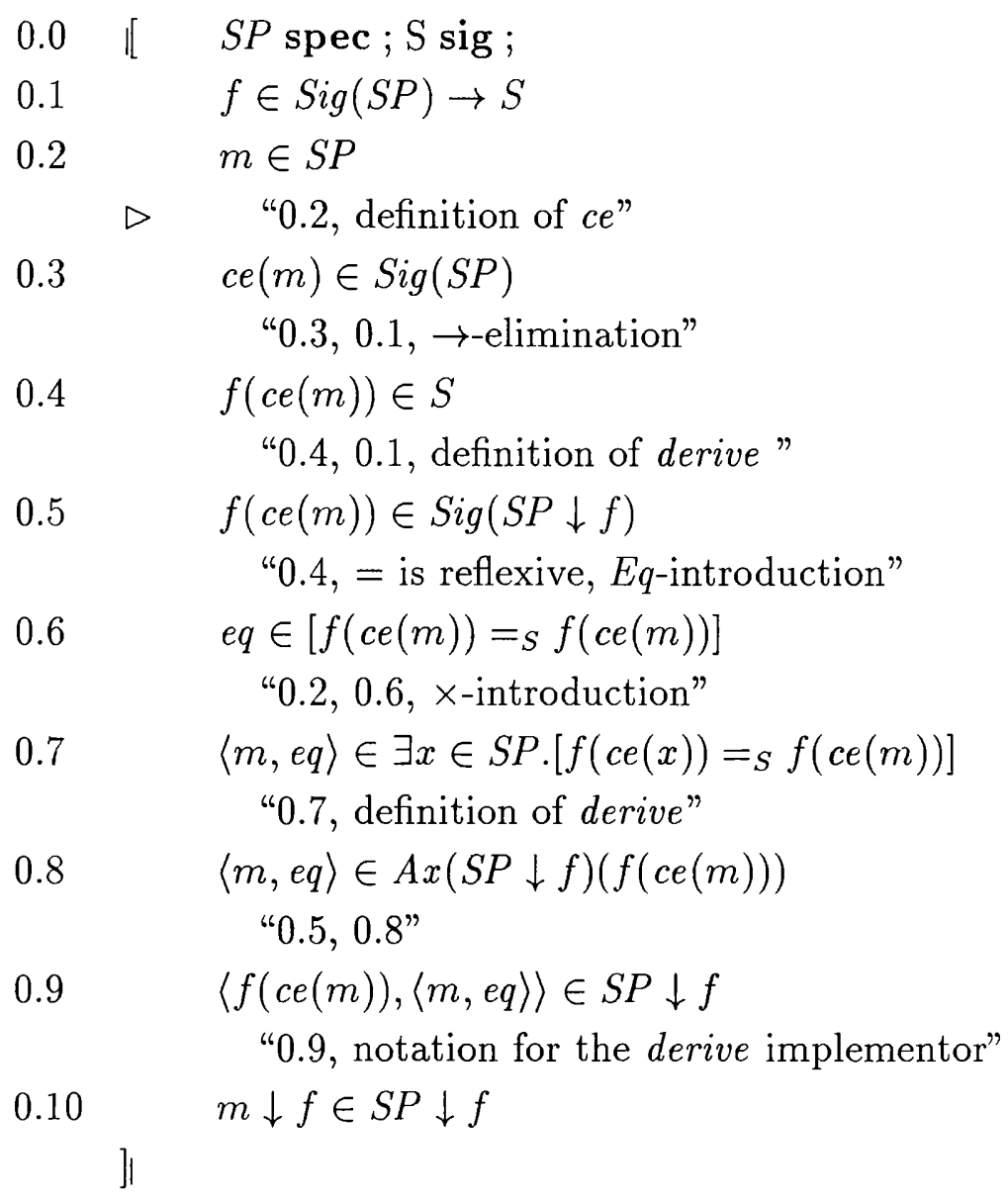

Figure 9.3: A proof of the Type Correctness of the Derive Implementor

\subsubsection{The Derive Implementor}

Let $S P$ be any specification, and $S$ any signature. An application of the derive implementor has the form $m \downarrow f$ for any module $m \in S P$ and any derive map $f \in \operatorname{Sig}(S P) \rightarrow S$. The module $m \downarrow f$ satisfies the specification $S P \downarrow f$. A formal definition of the derive implementor is given below:

\section{Definition 9.4 (The Derive Implementor)}

Given $S P$ spec, any $m \in S P$, and $f \in \operatorname{Sig}(S P) \rightarrow S$ where $S$ sig, the derive implementor $-\downarrow f \in S P \rightarrow S P \downarrow f$ is defined as follows:

$$
m \downarrow f=\langle f(c e(m)),\langle m, e q\rangle\rangle \in S P \downarrow f
$$

The computational element, $f(c e(m))$, of $m \downarrow f$ satisfies the signature, $S$, of $S P \downarrow f$. The expression $\langle m, e q\rangle$ is the witness of $m \downarrow f$. This witness satisfies the restriction of $S P \downarrow f$ which is $\exists x \in S P$. [f(ce(x))=s ce(m)]. Figure 9.3 contains a proof that $m \downarrow f \in S P \downarrow f$. 


\subsection{Some Implementation Laws}

Specifications that take the form of a union, enrich or translate operation cannot always be implemented in a piecewise fashion. In this section, we highlight the problem of implementing such specifications, and give some laws that can be used to implement them.

\subsubsection{Implementing Enrich}

Consider the specification $S P=S P_{1} \triangleleft \Lambda\langle S \mid R\rangle$. To implement $S P$ in a piecewise manner, we would like to develop some implementation $m_{1} \in S P_{1}$, and then add to $m_{1}$ an implementation for the components specified by the enrichment $\Lambda\langle S \mid R\rangle$. Our aim is to use $m_{1}$ to make an implementation for the components specified by $\Lambda\langle S \mid R\rangle$. However, it is possible that some implementations of $S P_{1}$ may make the restriction $R$ inconsistent, as they may be implemented without considering the extra restrictions on $S P_{1}$ introduced by $R$. Consequently, an unfortunate choice of implementation for $S P_{1}$ may prevent the implementation of the components specified by $\Lambda\langle S \mid R\rangle$. To show that an implementation of $S P_{1}$ avoids such "dead-end" developments requires proving that the remaining implementation task is consistent. It can be shown-and this is one of the interesting features of using type-theory to develop programs - that to prove that $R$ is consistent, for an arbitrary implementation of $S P_{1}$, is equivalent to making an implementation for the components specified by $\Lambda\langle S \mid R\rangle$.

Given an implementation of $S P_{1}$, the next stage in implementing $S P$ is to implement the components specified by $\Lambda\langle S \mid R\rangle . \Lambda\langle S \mid R\rangle$ can almost be viewed as a specification; although, it is not a specification since it is dependent on the components specified by $S P_{1}$. However, $\Lambda\langle S \mid R\rangle$ can be transformed into a specification by replacing the free names of components from $S P_{1}$ by actual implementations of such components taken from any implementation of $S P_{1}$. Given any $m_{1} \in S P_{1}$, we may implement the components of $\Lambda\langle S \mid R\rangle$ by implementing the specification $S P_{2}=\langle S \mid R\rangle\left(y_{1} \backslash m . y_{1}, \ldots, y_{n} \backslash m . y_{n}\right)$ where $y_{1}, \ldots, y_{n}$ are the names of the components in $m_{1}$ (and $S P_{1}$ ). Given any $m_{2} \in S P_{2}$, we can show that combining $m_{1}$ and $m_{2}$, using the sum implementor, gives $m_{1}+m_{2} \in S P$. We state this property, formally, in the following theorem, with an explanation following the theorem:

\section{Theorem 9.1}

Let $S_{1}=S i g\left(S P_{1}\right)$. If $m_{1} \in S P_{1}$ and $m_{2} \in\left\langle S\left(\mid c e\left(m_{1}\right)\right)_{S_{1}} \mid(m) R\left(c e\left(m_{1}\right) \oplus m\right)\right\rangle$ then:

$$
m_{1}+m_{2} \in S P_{1} \triangleleft \Lambda\langle S \mid R\rangle
$$

Proof Given in Figure 9.4 


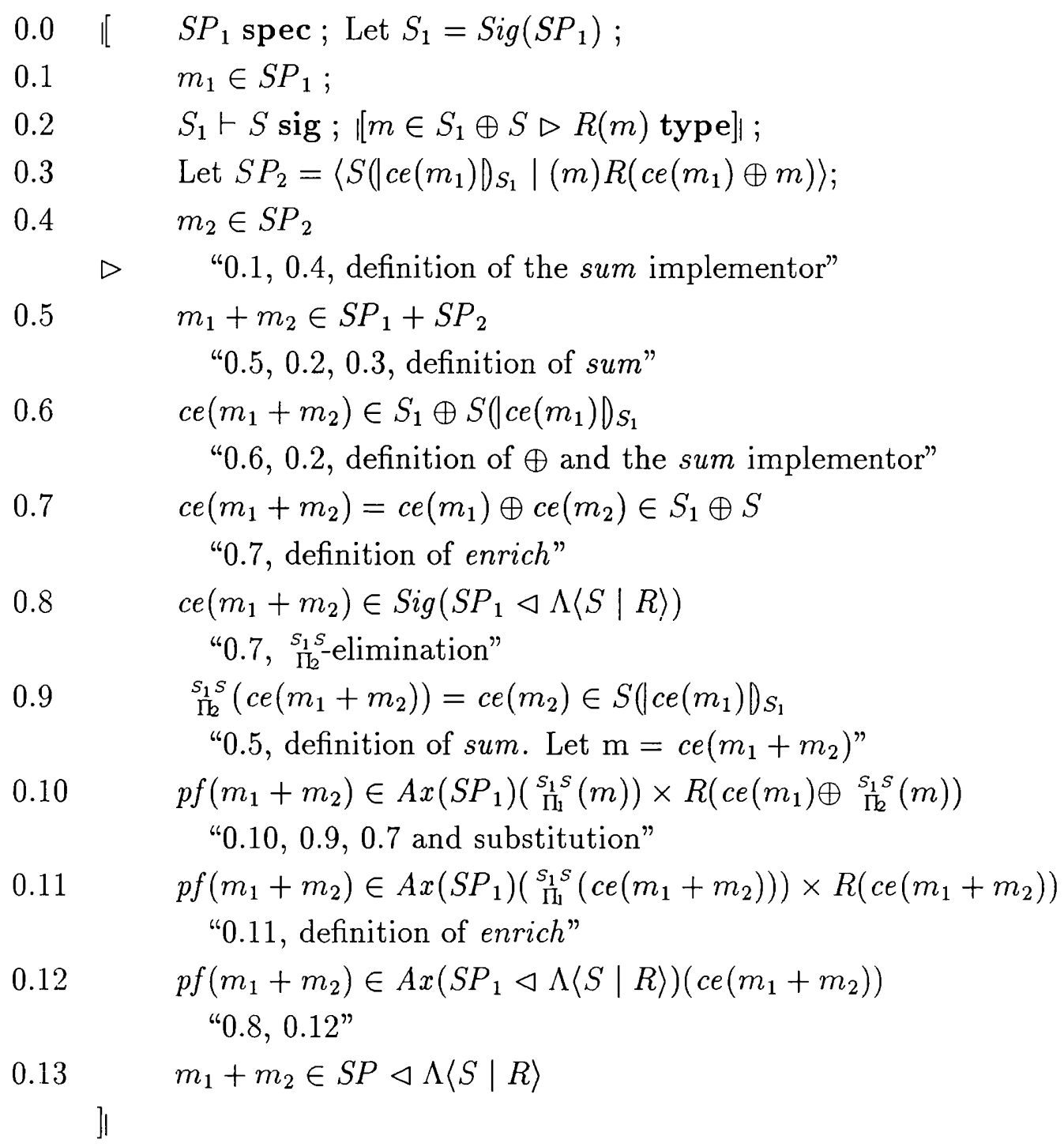

Figure 9.4: A proof of Theorem 9.1

In Theorem 9.1, the specification $\left\langle S\left(\mid c e\left(m_{1}\right)\right)_{S_{1}} \mid(m) R\left(c e\left(m_{1}\right) \oplus m\right)\right\rangle$, which we will refer to as $S P^{+}$, is equivalent to $S P_{2}$ above. In $S P^{+}$, the substitutions described in $S P_{2}$ are performed using the signature instantiation notation (Section 6.2.2), i.e. $S\left(\mid c e\left(m_{1}\right)\right)_{S_{1}}$ is $S$ with free occurrences of component names from $S_{1}$ replaced by implementations of such components taken from $c e\left(m_{1}\right) \in S_{1}$. By the definition of enrich, $R$ is dependent on computational elements with signature $S_{1} \oplus S$. The expression $R\left(c e\left(m_{1}\right) \oplus m\right)$, where $c e\left(m_{1}\right) \oplus m \in S_{1} \oplus S$, is $R$ with free occurrences of component names from $S_{1}$ replaced by implementations of such components taken from $c e\left(m_{1}\right) \in S_{1}$. Therefore, $(m) R\left(c e\left(m_{1}\right) \oplus m\right)$ is dependent on $m \in S\left(\mid c e\left(m_{1}\right)\right)_{S_{1}}$. 
Sometimes, the enrichment $S P=S P_{1} \triangleleft \Lambda\langle S \mid R\rangle$ can be implemented by a function that takes any implementation of $S P_{1}$ and returns an implementation of the components specified by $\Lambda\langle S \mid R\rangle$. The type of such a function is a dependent function type that we name $F$ :

$$
F=\prod x \in S P_{1} \cdot\left\langle S\left(\left|c e(x) D_{S_{1}}\right|(m) R(c e(x) \oplus m)\right\rangle\right.
$$

$F$ may be regarded as the specification of a parameterised module; in other words, the members of $F$ are functions that return modules. Given a member of $F$, it can be used to make an implementation of $S P$ :

\section{Theorem 9.2}

Let $S_{1}=\operatorname{Sig}\left(S P_{1}\right)$ and $F=\prod x \in S P_{1} \cdot\left\langle S(\mid c e(x))_{S_{1}} \mid(m) R(c e(x) \oplus m)\right\rangle$. If $f \in F$ and $m_{1} \in S P_{1}$ then:

$$
m_{1}+f\left(m_{1}\right) \in S P_{1} \triangleleft \Lambda\langle S \mid R\rangle
$$

Proof Similar to that of Theorem 9.1 above $\square$

In practice, $F$ is often inconsistent (i.e. has no members) since, as discussed earlier, some $m_{1} \in S P_{1}$ may make $R$ inconsistent, so that $F$ becomes inconsistent. Therefore, it is often easier to borrow a specific $m_{1} \in S P_{1}$-as in Theorem 9.1, above-to implement an enrichment.

In summary, we can implement specifications of the form $S P_{1} \triangleleft \Lambda\langle S \mid R\rangle$ in a piecewise manner, provided that we keep one eye on the restriction $R$ when implementing $S P_{1}$, to ensure that the implementation of $S P_{1}$ keeps $R$ consistent.

\subsubsection{Implementing Union}

Consider the specification $S P=S P_{1} \cup S P_{2}$ where we assume that $\operatorname{Sig}\left(S P_{1}\right)=$ $\operatorname{Sig}\left(S P_{2}\right)$. By the definition of union, the signature of $S P$ is $\operatorname{Sig}\left(S P_{1}\right)$, and implementations of $S P$ satisfy the restrictions of $S P_{1}$ and $S P_{2}$. Suppose we develop implementations $m_{1} \in S P_{1}$ and $m_{2} \in S P_{2} . m_{1}$ and $m_{2}$ both satisfy the signature of $S P$, but $m_{1}$ is not guaranteed to satisfy the restriction of $S P_{2}$; similarly, $m_{2}$ is not guaranteed to satisfy the restriction of $S P_{1}$. In general, there is no way to combine $m_{1}$ and $m_{2}$ to produce a module satisfying the restrictions of $S P_{1}$ and $S P_{2}$. Consequently, there is no point in implementing $S P$ in a piecewise manner.

In practice, $S P$ can be implemented by first unfolding it to eliminate the union operator, and then implementing the resultant specification. An alternative implementation strategy is to make an implementation $m_{1} \in S P_{1}$ and verify that it satisfies the restriction of $S P_{2}$ (i.e. verify that there exists some witness $\left.p \in A x\left(S P_{2}\right)\left(c e\left(m_{1}\right)\right)\right)$ : 


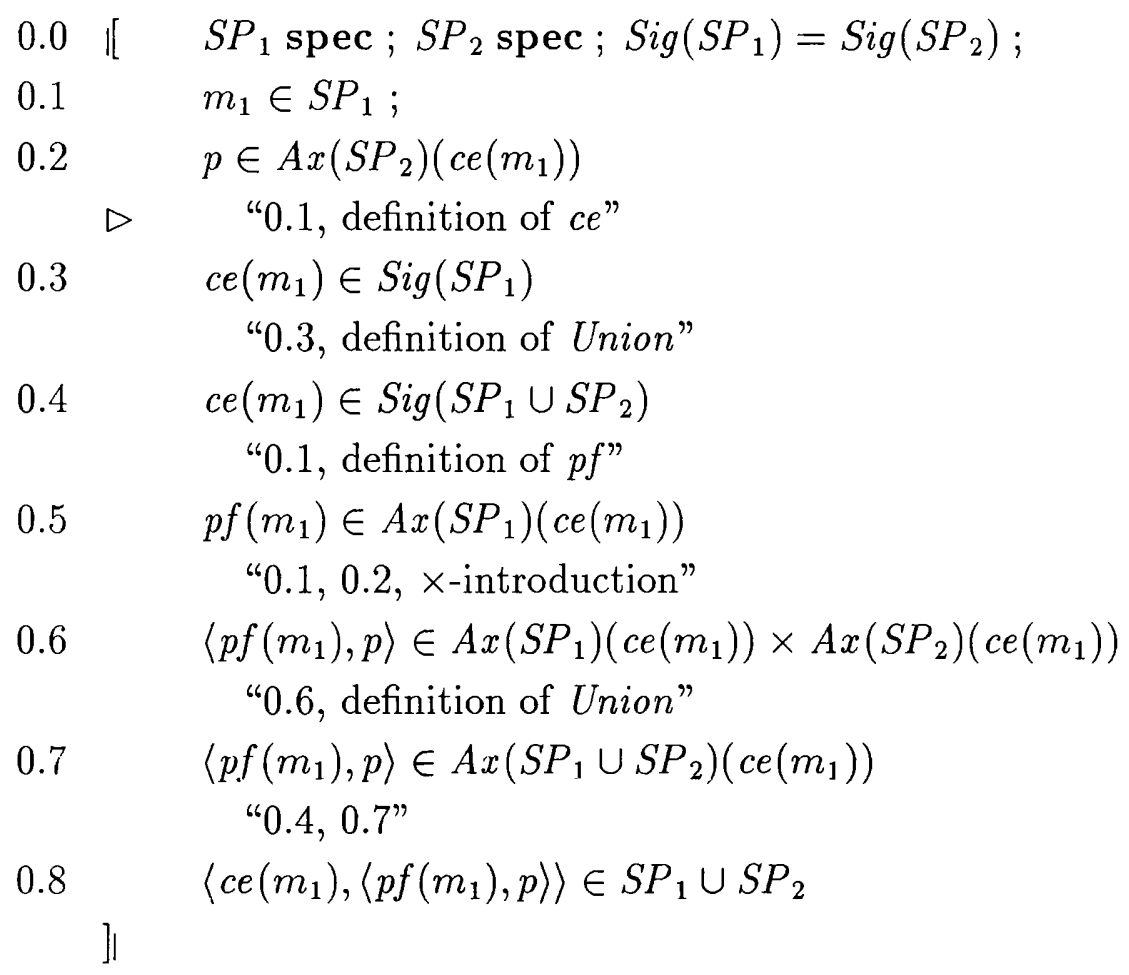

Figure 9.5: A Proof of Theorem 9.3

\section{Theorem 9.3}

Given any $m_{1} \in S P_{1}$, and any $p \in A x\left(S P_{2}\right)\left(c e\left(m_{1}\right)\right)$ then:

$$
\left\langle c e\left(m_{1}\right),\left\langle p f\left(m_{1}\right), p\right\rangle\right\rangle \in S P_{1} \cup S P_{2}
$$

\section{Proof Given in Figure $9.5 \square$}

We can also implement $S P$ by making an implementation $m_{2} \in S P_{2}$ and verifying that it satisfies the restriction of $S P_{1}$.

It could be argued that the union operator is of limited use since specifications that use it cannot be implemented in a piecewise manner. However, the union operator is extremely useful for making specifications and we should continue to use it in such a role. We should not let concerns about the implementation task interfere with the specification task. However, when we develop an implementation from a specification, the evidence above suggests that occurrences of the union operator should be eliminated during the refinement task. Applying such a heuristic makes it more likely that refinements can be implemented in a piecewise manner. 




Figure 9.6: A proof of Theorem 9.4

\subsubsection{Implementing Translate}

Consider a specification $S P=S P_{1} \uparrow f$ where $f \in S \rightarrow \operatorname{Sig}\left(S P_{1}\right)$ and $S$ sig. By the definition of translate, any implementation $m \in S P$ meets the signature $S$, and satisfies the restriction $A x\left(S P_{1}\right)(f(c e(m)))$. We can implement $S P$ in a piecewise manner provided that the translate map $f$ has an inverse:

\section{Theorem 9.4}

Given any $m \in S P$ and $f \in S \rightarrow \operatorname{Sig}(S P)$ where $S$ sig, if $f^{-1}$ exists then:

$$
\left\langle f^{-1}(c e(m)), p f(m)\right\rangle \in S P \uparrow f
$$

\section{Proof Given in Figure 9.6}

Not all translate maps have an inverse, so specifications such as $S P$ cannot always be implemented in a piecewise manner. If translate map $f$ has no inverse, $S P$ can be implemented by first unfolding it, to eliminate the translate operator, and then implementing the resultant specification. When refining a specification, we should attempt to eliminate all uses of the translate operation, except for those uses whose associated translate map has an inverse. Such a refinement heuristic makes it more likely that refinements can be implemented in a piecewise manner. 


\subsection{An Example Implementation}

In this section, we give an example of implementing a specification. We choose to implement the specification $\mathrm{Mean}_{3}$ which we developed in Section 8.7 as a refinement of the specification Mean-Mean and $\mathrm{Mean}_{3}$ are given in Figures 3.1 and 8.4, respectively. Recall that $\mathrm{Mean}_{3}$ specifies operations to calculate the mean of a sample of naturals, and is defined as follows:

$$
\text { Mean }_{3}=\text { PointSpec }_{2}[\text { Point } \backslash \text { Pair }] \triangleleft \text { Mean }^{+}
$$

where the details of PointSpec $_{2}$ and the enrichment Mean ${ }^{+}$can be found in Figure 8.4. We will show that the implementors and implementation laws given in this chapter can be used to implement $\mathrm{Mean}_{3}$ in a piecewise manner.

In the following, we will abbreviate PointSpec ${ }_{2}[$ Point $\backslash$ Pair $]$ as $P S_{2}$, and the signature and restriction of $\mathrm{Mean}^{+}$as $S$ and $R$, respectively.

\subsubsection{The Implementation of $\mathrm{Mean}_{3}$}

The structure of $\mathrm{Mean}_{3}$ suggests using Theorems 9.1 or 9.2 as a starting point for its implementation. In fact, we will use Theorem 9.2 and implement $\mathrm{Mean}^{+}$by a function developed independently of any implementation of $P S_{2}$. We will implement the enrichment $M e a n^{+}$first, and then implement $P S_{2}$.

To implement $\mathrm{Mean}^{+}$by Theorem 9.2 , we require to construct a function that has the following type:

$$
F=\prod x \in P S_{2} \cdot\left\langleS \left(|c e(x)|_{P S_{2}}|(m) R(c e(x) \oplus m)\rangle\right.\right.
$$

We omit the details of making a function of type $F$, but the restriction, $R$, of $\mathrm{Mean}^{+}$ strongly suggests the function $f$, given in Figure 9.7, as a member of $F . f$ may be developed through a constructive proof from its type $F$; the proof is largely a clerical task.

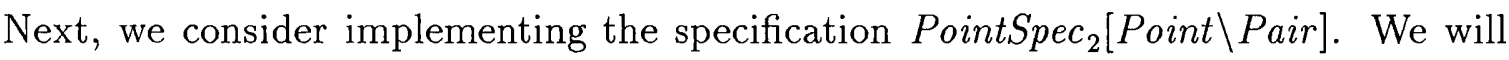
implement PointSpec 2 , and then apply the rename implementor to its implementation to produce an implementation of PointSpec $_{2}[$ Point $\backslash$ Pair $]$. The restriction of PointSpec $_{2}$ suggests the module PointModule, in Figure 9.8, as an implementation of PointSpec $_{2}$. We can verify that

$$
\text { PointModule } \in \text { PointSpec }_{2}
$$

by a constructive proof; this task is a largely clerical and is omitted. By applying the rename implementor to PointModule we get:

$$
\text { PointModule }[\text { Point } \backslash \text { Pair }] \in \text { PointSpec }_{2}[\text { Point } \backslash \text { Pair }]
$$




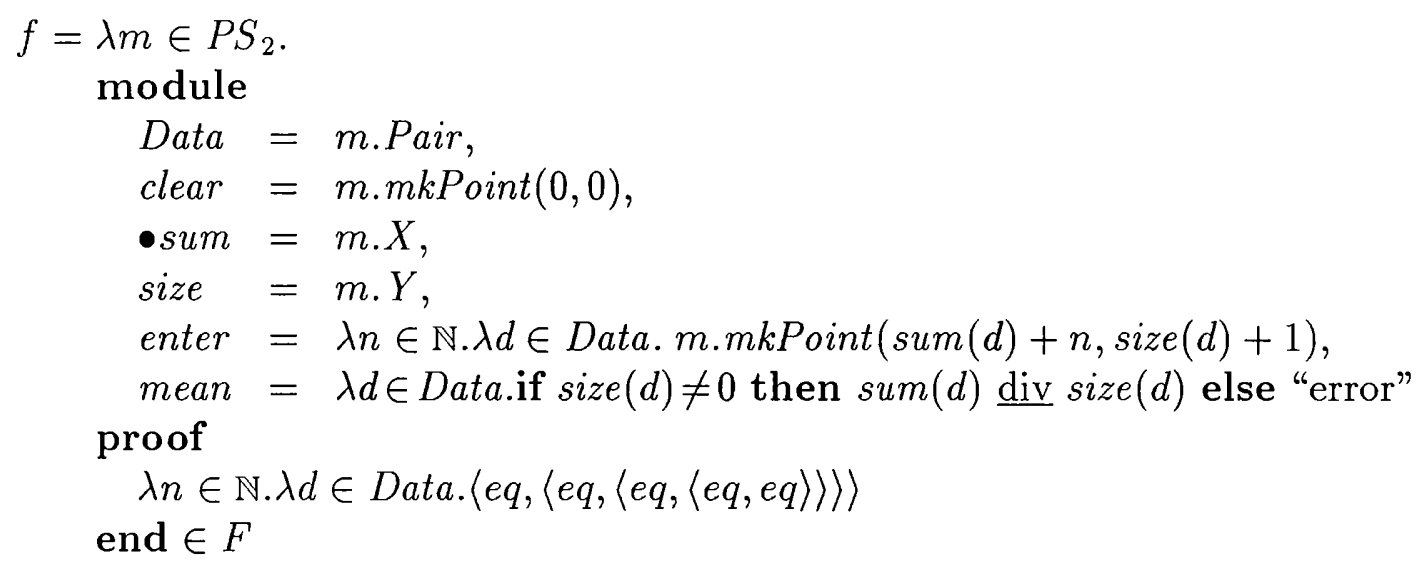

Figure 9.7: A parameterised module of type $F$

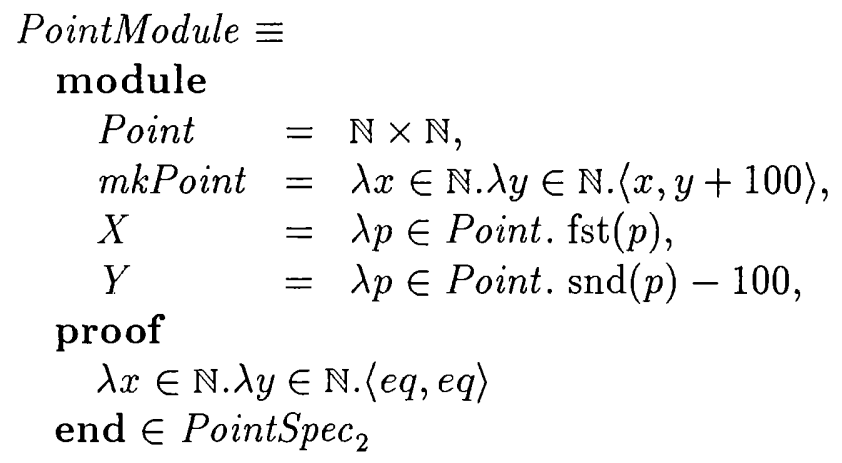

Figure 9.8: An implementation of PointSpec $_{2}$

We put all the implemented pieces together by applying Theorem 9.2 to get the following implementation, named MeanModule, of $\mathrm{Mean}_{3}$ :

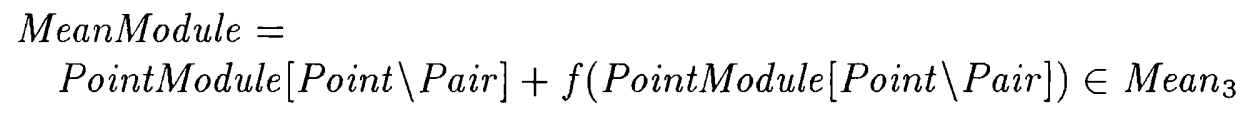

\subsubsection{A Discussion of the Example}

We have deliberately used the structure of $\mathrm{Mean}_{3}$ to guide its implementation, and have succeeded in our goal of developing the implementation in a piecewise manner. Using the structure of a specification to guide its implementation often simplifies the implementation task. This statement is justified, to some extent, by our example. Of course, we assume that the structured specifications we implement have already been refined, so the task of implementing its constituent canonical specifications is relatively straightforward; implementing un-refined specifications is usually more difficult than implementing refined specifications.

Making implementations through implementors and functions has some interesting consequences. One of these is that if we reuse existing specifications to make a new 
specification, then it may also be possible to reuse their implementations to implement the new specification. This is illustrated in our example, which shows how reusing PointSpec to make $\mathrm{Mean}_{3}$ allows us to reuse the implementation PointModule to implement $\mathrm{Mean}_{3}$. This property is a direct consequence of having laws to refine and implement specifications in a piecewise manner.

The observant reader will have noticed that PointModule appears twice in MeanModule. By abstracting over PointModule, we can develop a parameterised module that returns different implementations of $\mathrm{Mean}_{3}$. For example, function $g$, below, takes any implementation of PointSpec 2 and returns an implementation of $\mathrm{Mean}_{3}$ :

$$
g=\lambda m \in \text { PointSpec }_{2} . m[\text { Point } \backslash \text { Pair }]+f(m[\text { Point } \backslash \text { Pair }])
$$

where

$$
g \in \text { PointSpec }_{2} \rightarrow \mathrm{Mean}_{3}
$$

The type of $g$ is justified by the definition of the rename implementor and Theorem 9.2. The function $g$ is a parameterised version of MeanModule, and it can be used to express MeanModule as follows:

\section{MeanModule $=g($ PointModule $) \in$ Mean $_{3}$}

However, the main advantage of defining $g$ is that any implementation of PointSpec 2 may be used to produce an implementation of $\mathrm{Mean}_{3}$. In other words, if we subsequently choose to re-implement PointSpec $_{2}$ - maybe for reasons of efficiency - then $g$ allows us to reuse $f$ to make a new implementation of $M e a n_{3}$. The fact that we can reuse implementations in this way is a direct consequence of using a structured approach to construct, refine and implement specifications.

\subsection{Discussion and Summary}

The stepwise implementation of specifications of modules is also encouraged in the refinement calculus [39] and algebraic specifications [50, 56]. It is worth pointing out that our notion of implementation differs from that commonly used by algebraic specifications. Typically, algebraic specifications are implemented by other algebraic specifications whose axioms look like programs. However, there is work on implementing algebraic specifications as programs. In particular, [50] implements algebraic specifications via functions called constructors. Our use of implementors is similar to the notion of constructors in [50]. In Extended ML [48], algebraic specifications are implemented as programs in the functional programming language Standard ML.

There are several advantages to a stepwise approach to implementation. We avoid rearranging a specification prior to, and during, implementation. It also makes it more likely that we can reuse implementations of existing specifications. For example, 
consider the specification $S P_{1}+S P_{1}[p \backslash q]$. If we make an implementation $m_{1} \in S P_{1}$, we can reuse $m_{1}$ to make the implementation $m_{1}[p \backslash q] \in S P_{1}[p \backslash q]$ and, by the sum implementor, we get $m_{1}+m_{1}[p \backslash q] \in S P_{1}+S P_{1}[p \backslash q]$. Piecewise implementation also helps to reduce the amount of recoding necessary if we change part of a specification. For example, consider the implementation $m_{1}+m_{2} \in S P_{1}+S P_{2}$. If we change $S P_{2}$ to any specification $S P_{3}$ then we only have to recode $S P_{3}$ to get an implementation of $S P_{1}+S P_{3}$; for any $m_{3} \in S P_{3}$ we get that $m_{1}+m_{3} \in S P_{1}+S P_{3}$.

Piecewise implementation does have limitations. Making an implementation with the same structure as its specification may not lead to an efficient implementation. If we implement part of a specification in isolation, without considering how it interacts with the rest of the specification, then we could preclude efficient implementations that might be obtained by rearranging the specification prior to implementation. However, large specifications can be difficult to rearrange, and it is usually easier to implement them directly in a piecewise manner rather than rearrange them. In [13] - which discusses the implementation of modules in VDM-it is argued that the structure of a specification should be designed to make a specification easier to understand, rather than easier to implement. We agree with this argument, and it does not preclude the piecewise implementation of specifications.

We have only given the most basic theorems about the piecewise implementation of structured specifications, and they are in no way complete. In particular, each implementor has many algebraic properties that may help in making implementations. In general, the algebraic properties of each implementor are similar to the properties of the computational element operator used to define the implementor. For example, the sum implementor is associative since computational element concatenation is associative. We have not defined the algebraic properties of implementors since we have not required their use, but such properties are not difficult to prove.

In summary, we have shown that in type theory specification can be implemented in a piecewise manner. We have defined some module operators, called implementors, and used them to give some implementation laws that aid the piecewise implementation of specifications. 


\section{Chapter 10}

\section{Conclusions}

The need to apply formal specification and development of programs to large problems has highlighted a need for methods to support the modular construction of specifications and their implementations. Martin-Löf's Type Theory supports both these activities. Type theory allows us to express specifications, modules and specification building operators in a single framework. Furthermore, we can incrementally construct and implement modular specifications in the single framework of type theory.

The main conclusions of this thesis are that, in principle, Martin-Löf's Type Theory can be used to express a rich specification and implementation language for modules, and it also provides a framework for the incremental construction and implementation of modular specifications.

In this chapter, we summarise the results of the main chapters in this thesis: Chapters $3,4,6,7,8$ and 9 . We also give a global critical review of Martin-Löf's Type Theory for specification and programming, and our use of the theory in this thesis.

\subsection{Summary}

\subsubsection{Typeful Specification}

Adopting a type-theoretic view of modules and their specifications allows us to exploit many of the features of type theory when making specifications. In Chapter 3 we described the advantages of typeful specification. The main conclusion of Chapter 3 is that typeful specification simplifies the specification task; it produces more concise specifications, and it allows specifications to be made in a hierarchical manner that makes specifications easier to understand.

It was shown that we can use the built-in types in Martin-Löf's Type Theory as models for data-types specified in our specifications. This approach contrasts with 
the purely property-based style of specification encouraged by algebraic specification languages, in which we may not assume any model for data-types (sorts). We gave empirical evidence that specifying models for data-types results in specifications with fewer axioms, compared with equivalent property-based specifications. Consequently, it is easier to verify that a model-based specification specifies the system we intended it to specify, compared to an equivalent property-based specification.

The fact that specifications are types allows us to use specifications as types for components in specifications. This allows us to nest one specification inside another. We say that such a style of nesting is hierarchical as it introduces different scopes, and so, components with the same name can co-exist in the same specification.

Interpreting specifications as types allows us to use specifications as record types, and create multiple instances of a module inside a specification. This is shown to be useful when specifying complicated data-structures; we gave an example of a book library that uses multiple instances of a book module to represent the stock of a library.

We defined parameterised specifications as functions that return specifications. By specifying the parameter of a parameterised specification to be a type, we can specify generic specifications that can be instantiated with different types. We may also use modules as parameters, so that we can use the operations in a module to make a specification; in other words, we can mix specifications and implementations to make specifications.

It was shown that by using the fact that propositions are types, we can add equality relations-called observational equalities-as components in specifications, and that these relations can be used to solve the problem of over-specifying modules. Adding observational equalities to a specification adds extra restrictions to it, but allows more implementations to be derived from a specification.

\subsubsection{The Semantics of Specifications}

To be useful, a specification language must have a formal semantics. A formal semantics allows us to reason about specifications, and to justify laws for developing implementations from specifications. In Chapter 4, we defined a semantics for canonical specifications, and modules, in Martin-Löf's Type Theory. A specification is a type, and its members are modules that satisfy it.

We reviewed Nordström and Petersson's (NPS) approach to specifying modules in type theory, and contrasted this with Burstall's Deliverables approach to making specifications in type theory. We concluded that both the above approaches were deficient since they do not include a formal semantics for the name-space of components in modules and specifications. 
We proposed, and defined, a semantics for specifications and modules which is a fusion of the NPS and Deliverables approaches, but where component names have a formal semantics. We also gave local components a formal semantics in specifications and modules.

Many specifications that we would expect to be equal to each other are not equal in type theory. It was shown that we can define a specification equality, based on a weak equality on types, that admits equalities not allowed by type equality.

\subsubsection{Structured Specifications}

One way of making large specifications is to construct them by combining smaller specifications. Such a style encourages us to make specifications incrementally, as well as helping to decompose the specification task into more manageable pieces. Chapters 6 and 7 are concerned with defining operators that allow us to combine and modify specifications to produce structured specifications. The main conclusion of Chapters 6 and 7 is that it is possible to define a useful collection of specification operators for a modular specification language in the framework of Martin-Löf's Type Theory.

In Chapter 6, we defined renaming, hiding and concatenation operators on signatures and computational elements; these operators were used in Chapter 7 to define structuring operators on specifications. An important achievement in Chapter 6 was the definition of a signature concatenation operator that resolved the name-clashes that can arise when combining specifications; we showed that allowing overloading of component names provided a simple mechanism for resolving name-clashes.

An important result in Chapter 6 was the relationship shown to exist between each signature operator and the corresponding computational element operator: given any signature $S$ and computational element $m \in S, f(m) \in F(S)$ for any signature operator $F$ and its corresponding computational element operator $f$. This relationship was exploited in Chapter 9 to give laws for implementing structured specifications.

In Chapter 7, we proposed a collection of specification operators that can be used to construct structured specifications. It was shown, by example, that the specification operators can be used to construct specifications in an incremental style, by adding more and more detail to a specification until we are satisfied that it specifies what we want. It was also shown that an incremental style of construction confers some benefits in the area of reusing specifications.

We formulated some laws that, under certain conditions, allow us to simplify the semantics of structured specifications; these laws aid reasoning about structured speci- 
fications. It was shown that the specification operators enjoy a collection of algebraic properties that can be used to reason about, and rearrange, structured specifications.

\subsubsection{Refinement}

One of the attractions of using type theory to construct specifications is that it can also be used to make implementations from specifications. Since specifications and modules are defined in the single framework of type theory, we can give a precise meaning to a notion of refinement that supports the implementation of specifications by mathematical transformation. In Chapter 8 , we gave a formal definition of refinement for specifications in type theory, together with a collection of refinement laws. The main conclusion of Chapter 8 is that type theory provides an excellent framework for the systematic refinement of large structured specifications.

We have shown that our definition of refinement allows the piecewise refinement of specifications. That is to say, we can refine a specification by refining its individual parts more or less independently of each other, and then combine its refined parts to get a refinement of the whole specification. The piecewise refinement property is due to the monotonicity of our specification operators; we gave proofs of the monotonicity of each of our specification operators. We have also shown that refinement is transitive. The transitivity of refinement, together with the monotonicity of our operators, allow us to refine a specification in small steps, such that a derived specification is always a refinement of the original.

We stated, and proved, some refinement laws that allow large unstructured specifications to be refined by structuring them using the specification operators.

We showed that all types, not just specifications, can be refined in type theory. Moreover, we have shown that the types of the individual components in the signature of a specification can be refined to produce a refinement of the whole specification.

\subsubsection{Implementation}

Once we have refined a specification, we then implement the specification. The restriction of a canonical specification usually suggests an implementation, and making such an implementation is usually straightforward. However, implementing structured specifications is more complicated, and is discussed in Chapter 9.

We defined a collection of module operators, called implementors, that correspond to some of our specification operators. It was shown that we can often implement a structured specification by implementing its constituent canonical specifications, and 
replacing the specification operators used in the specification with their corresponding implementors. In other words, we can use implementors to implement structured specifications in a piecewise manner.

Piecewise implementation is shown to have its limitations; for example, we show that enrich and union operations cannot always be implemented in a piecewise manner. Furthermore, making an implementation with the same structure as its specification may not always lead to an efficient implementation. However, piecewise implementation is still a useful strategy, as it decomposes the implementation task into more manageable pieces. It was also shown that piecewise implementation encourages the reuse of implementations; and this helps to simplify the implementation task.

The main conclusion of Chapter 9 is that defining specification and module operators in the single framework of type theory allows us to implement structured specifications in a piecewise manner.

\subsection{A Critical Review of MLT}

In this thesis, we have shown that Martin-Löf's Type Theory (MLT) provides a more than adequate framework for constructing modular specifications and programs. We have found MLT pleasurable to use. The rules of the type theory have a logical structure that makes them easy to master, and the formal language of the type theory is very expressive as a specification and programming language. However, MLT has some drawbacks that limit its use as a specification and program development framework. In this section, we address some of the drawbacks of MLT. We give a critical appraisal of MLT for specification and programming in general, and of the particular approach taken in this thesis.

\subsubsection{Specification and Programming in MLT}

We begin by considering the intuitionistic logic used by Martin-Löf's Type Theory. We have found intuitionistic logic more difficult to use and reason about compared to classical logic. The fact that many propositions that are equal to each other in classical logic are not equal under intuitionistic logic means that specifications that we would expect, and desire, to be equivalent to each other are not equal in type theory. We overcame this problem by defining weak type equality $(\Leftrightarrow)$ to compare propositions. However, the introduction of weak type equality imposes an overhead on specifiers and implementors, as they must reason about normal type equality and weak type equality in order to compare specifications. 
The need for weak type equality and specification equality is an indication that in many instances the identification of propositions with types is far-fetched and awkward. This identification may result in computationally irrelevant proof objects appearing in programs derived from specifications in MLT. To reason about the computationally relevant parts of such programs, it is often necessary to separate the computational parts and proof objects. This is an irritating overhead when reasoning about programs in MLT. Consequently, there has been a trend towards separating propositions from types when making specifications in MLT and other type theories. Indeed, the Calculus of Constructions, and the Extended Calculus of Constructions both make a formal distinction between types and propositions. Our specifications of modules also separate data-types from propositions, by putting data-types in the signature, and propositions in the restriction of a specification. We have found that with such a separation, proof objects do not interfere with the derivation of modules, or reasoning about them. However, ensuring such a separation still adds to the work of a specifier, and we can make some specifications more easily by mixing propositions and data-types.

Subset types have been introduced into MLT in order to avoid computationally irrelevant proof objects appearing in programs. However, subset types do not integrate well with the principle of propositions as types because they throw away proof objects. The loss of proof objects makes it difficult to deduce properties about specifications that use subset types; we discussed this in Section 4.10.2. It seems that if we want a subset type that will work in practice, it must be possible to say that a proposition is true without explicitly showing a proof object. Nordström et al have obtained this by defining the subset theory [44]. The subset theory is an extension of MLT with two new forms of judgement $P$ prop and $P$ true, which mean that $P$ is a proposition and $P$ is true, respectively. In the subset theory, the logical constants-such as $\wedge$ and $\vee$-are no longer abbreviations for type constructors, but are introduced as new primitive constants. Moreover, propositions are no longer identified with types. It seems that the price we have to pay to introduce usable subset types is a more complicated theory.

Related to the identification of propositions with types is a potential to confuse propositions with Boolean values in MLT. This is not a serious problem, but it can make it hard to express some propositions succinctly. For example, $x<y(x, y \in \mathbb{N})$ is a Boolean expression where $<_{-} \in \mathbb{N} \rightarrow \mathbb{N} \rightarrow \mathbb{B}$, but to use $x<y$ as a proposition we have to write $\left[x<y=_{\mathbb{B}}\right.$ true $]$ which is rather cumbersome. Moreover, the effective use of Boolean values requires the definition of Boolean connectives, and these can be confused with propositional connectives. For example, $\left[x=_{\mathrm{N}} y\right]$ is a proposition (and type), but the expression $x=y$ is a Boolean expression when used in the expression if $x=y$ then 0 else 1 , where ${ }_{-}=\_\in \mathbb{N} \rightarrow \mathbb{N} \rightarrow \mathbb{B}$ is a Boolean connective. 
There are several problems with MLT that concern the expression of functions and types in the formal language of MLT. Firstly, general recursion is not available. Secondly, many useful functions that are pre-defined in most programming languages are not pre-defined in MLT. Thirdly, the theory does not include partial functions.

Many programming problems can be solved elegantly using recursive solutions, and this suggests that the introduction of general recursion to MLT is an urgent problem. The unavailability of general recursion means that the definitions of many recursive programs in MLT are more complicated than the definitions of the same programs in programming languages that allow general recursion. At present, we can only define recursive programs by using the primitive recursive operators, such as natrec and listelim, together with higher order functions. In our experience, such functions are difficult to define and reason about. The introduction of general recursion would not only simplify the definition of such functions, but would also make it easier to derive recursive programs from specifications in MLT. However, the introduction of general recursion to the theory is still an open problem, and is discussed in [45] and [42].

Some functions that we commonly use to make specifications and programs are not pre-defined in MLT. Such "auxiliary" functions include arithmetic operators such as sum, subtraction, div, mod; Boolean functions such as equality tests between naturals, ordering relations such as _ < ; and list operations such as head, tail, concatenation, etc. A programmer must define and, if necessary, deduce the properties of any auxiliary function he or she needs to use. This can add extra work to a specification or implementation task. We admit that defining most auxiliary functions is not intellectually taxing to the practising programmer; although some functions may be difficult to define due to the lack of general recursion. Nevertheless, the need to give the definitions of auxiliary functions can clutter specifications and programs in MLT.

The requirement that all functions be total in MLT has only been a problem to us when defining functions on types. Most of the functions we have defined on types are applied to $\sum$-types. To make such functions total, we have to define their domain to be $U_{1}$, even though we only apply the functions to $\sum$-types; this is because there is no kind in MLT that contains only $\sum$-types. It would be easier to define such functions as partial functions, if the theory allowed them. Constable et al [8] have introduced partial functions to the type theory used in the NuPRL system, and we regard that as a useful innovation to type theory.

The fact that specifications and programs inhabit different worlds in MLT causes some problems in using MLT as a program development framework. Firstly, it means we have to develop separate notations for specifications and programs; in this thesis, we have had to develop a specification language and an associated implementation language for modules. Secondly, it can affect the smooth derivation of programs since 
we have to jump from the world of types to the world of programs when making programs from specifications.

Although MLT suits the development of sequential functional programs, it is unsuited to the development of some other styles of programs that arise in the real world, such as imperative or concurrent programs. There is no formal notion of a state in MLT. Constable [9] suggests that state can be understood in type theory by manipulating $\mathrm{n}$-tuples of values as if they were the values stored in a state. For example, if a state contains the values $x \in \mathbb{N}$ and $y \in \mathbb{N}$ then the statement $y:=y+1$ is represented by the function $f(\langle x, y\rangle)=\langle x, y+1\rangle$. In practice, passing the state from one operation to the next is unwieldy, and it does not capture the idea of values being updated in place. Exceptions are another important programming language feature not available in MLT. However, Murthy [41] has shown that we can introduce control operators similar to exceptions into a constructive type theory. Therefore, in principle, exceptions could be added as an extension to MLT. We cannot make concurrent programs in MLT since all the operators in MLT are sequential, and functions can only be composed sequentially. This is a significant limitation to the usefulness of type theory for making programs in the real world, as the development of parallel computers has increased interest in making concurrent programs that exploit such hardware. Nondeterministic programs cannot be defined in MLT since all its operators are deterministic; the evaluation of terms in MLT is defined under a lazy and deterministic evaluation order. The lack of nondeterminism is a loss as it useful for making programs concerned with systems programming and scheduling resources. We can make nondeterministic specifications in MLT, but these must be implemented by deterministic programs. For example, the following specification does not determine the value of function $f$, but any implementation of $f$ will be deterministic:

Elements $f \in \mathbb{N} \rightarrow \mathbb{N}$ Restrictions $\forall x \in \mathbb{N} .0<f(x)<100$ end

\subsubsection{A Critical Appraisal of Our Approach}

We now give an overall critical appraisal of the particular approach to making specifications and programs taken in this thesis. Our main criticism concerns the complexity of the semantics of our specification language and its associated implementation language. The semantics are complicated to understand and reason about because we have had to introduce so much notation to handle them. One reason for so much notation is the lack of pre-defined operations in MLT. The unavailability of general recursion has added to the complexity of the notation. The complexity of the semantics has been exacerbated by the fact that we have had to define many operations twice-once for specifications and once for modules. This is a direct consequence of the fact that specifications and programs inhabit different worlds in MLT. 
The complexity of our semantics for specifications and modules adds to the difficulty of reasoning about, and proving properties of, specifications and programs. In particular, we found it hard to prove some of the algebraic properties of the specification operators. Indeed, we found it necessary to define some laws to simplify specifications made using the specification operators. We also found it necessary to give laws to simplify the restrictions of structured specifications because of the difficulties, discussed earlier, of reasoning in intuitionistic logic.

The fact that MLT distinguishes between specifications and programs has lead to our choice of a two-stage strategy for implementing specifications: first, we refine a specification, and then we find a module that implements its refinement. Therefore, we have developed two calculi for program development: one for refining specifications, and one for implementing refined specifications using our "implementor" functions. Consequently, a programmer is burdened by having to use, and master, two different calculi. To add to the programmer's burden, the two calculi employ different proof techniques. Refinement proofs employ equational reasoning, whereas proofs concerning the implementation of refined programs employ a constructive proof technique.

So far in this review, we have mentioned some of the limitations of using MLT to make specifications. However, in this thesis, we have also highlighted some special advantages of using type theory to make specifications. For example, dependent functions make it easy to return error messages. The theory also allows us the freedom to make specifications in an algebraic or model-oriented style as suits a specification problem. Furthermore, the use of types and specifications as first-class values make it easy to define parameterised specifications. There are other features of type theory that we have not exploited for making specifications. These include the use of higher order functions to describe restrictions; and the use of polymorphic types [3] to make specifications and programs.

We now compare the utility and elegance of our approach to specification and program development with other approaches such as Z, CLEAR, VDM, etc. The overall utility of our approach compares favourably against other modular approaches. Like Z and CLEAR, our approach encourages an incremental style of specification construction that is more than adequately supported by the operators we supply. Moreover, compared with $\mathrm{Z}$ or CLEAR, our approach offers more flexibility and ease in making structured specifications, because it allows us to structure specifications hierarchically by nesting specifications.

When it comes to specifying the individual structural units that make up a structured specification (i.e. canonical specifications in our approach, Schemas in Z, Theories in CLEAR, etc.) state based languages, like $\mathrm{Z}$ and VDM, have the advantage of allowing us to specify operations in an equational or imperative style; our approach only supports an equational style. Apart from our specification language, all the 
othcr specification languages mentioned above use classical logic. Therefore, because of the problems with intuitionistic logic that we discussed earlier, we sometimes find it harder to reason about specifications made in our specification language as compared to reasoning about similar specifications made in the other specification languages. However, it is often easier to rearrange and modify specifications in our approach because of the extra structuring techniques it offers.

It can be argued that VDM, Extended ML, CLEAR and the refinement calculus provide a more elegant approach to program development compared to ours, because in these languages the programming language is an implementable subset of the specification language. With such an approach, we can implement specifications by refinement alone; there is no need to jump abruptly from the world of specifications to the world of programs, as in our approach. Nevertheless, we have found our approach to implementing programs from specifications to be perfectly adequate.

\subsection{Specification in ECC and MLT: a comparison}

Luo has shown, in [27], that the Extended Calculus of Constructions (ECC) [24, 25, 26] provides an excellent framework for the modular specification and development of programs. He has shown this by defining specifications and specification building operators in ECC, as well as defining an effective notion of specification refinement in ECC. Luo's work tackles many of the issues discussed in this thesis. However, Luo's work is more general than ours: our work deals with many detailed issues such as component namespaces and local components, whereas Luo's work is more concerned with showing how the higher-order features of type theory provide useful mechanisms for structuring and implementing specifications. In addition, Luo investigates some issues, such as parameterisation, in more depth than we have discussed in this thesis. In this section, we discuss the points in common, as well as the differences, between Luo's work and ours.

In the following, we assume the reader is familiar with the basic features of ECC; recall that Section 1.1.3 contains a brief description of the Calculus of Constructions and ECC. For the purpose of comparison, ECC may be seen as an extension of MLT with an impredicative universe Prop of all propositions. There are minor differences in syntax between ECC and MLT. For example, type universes in ECC are written as Type $_{i}(i>0)$ and not $U_{i}$ as in MLT; in fact, Luo omits the universe level $i$ altogether and writes Type instead of Type $i$ since $i$ can be inferred from the context in which Type is used in ECC.

A specification in ECC is a pair, and not a type as in our work. The first component of a specification in ECC is any type $S \in$ Type, called the structure type of the 
specification, and the second component is any predicate $P \in S \rightarrow$ Prop on $S$. The structure type of a specification $S P$ plays the same role as a signature: it gives the type of the possible programs that may realise $S P$. The predicate part of $S P$ specifies the properties that programs realising $S P$ must satisfy. Note that, in common with our specifications in MLT, specifications in ECC keep their computational part (expressed by the structure type) and axiomatic part separate.

Notation For any specification $S P$ in ECC, we may write $\operatorname{Str}(S P)$ to mean the structure type of $S P$, and $A x(S P)$ to mean the predicate part of $S P$.

The decision to define specifications in ECC as pairs rather than $\sum$-types (as we do) is intentional. There are two reason for the decision. Firstly, it permits the definition of operations that decompose specifications in ECC into their structure type and axiomatic part (by using the functions $f s t$ and $s n d$ ). Such operations could not be defined in ECC if specifications in ECC were $\sum$-types since ECC does not have an operator like urec in MLT that defines operations over the structure of all types. Secondly, the definition of specifications as pairs means that it is possible to define a type, called $S P E C$, of all specifications in ECC: $S P E C=\sum S \in$ Type.S $\rightarrow$ Prop. In principal, we can define a type of all specifications in MLT by using the subset type constructor; but such a definition is of limited use due to the limitations of subset types discussed earlier (Section 4.10.2). SPEC is useful for defining specification operators and parameterised specifications, and we say more about it later.

Luo shows how to specify modules like ours in MLT, but without component names and local components, by making the structure type of a specification in ECC a loose signature. Unlike specifications in MLT, there is no formal mechanism for naming components or introducing local components in specifications in ECC. This is reflected by the fact that a structure type can be any type, whereas a signature in MLT is defined to include a semantics for the namespace of components. Consequently, Luo's work is free of much of the detail that the inclusion of a formal semantics for namespace introduces to our work. The fact that ECC and MLT supply the same logical connectives means that the predicates used in the axiomatic parts of specifications in ECC are similar in style to the predicates used as restriction types by our specifications. In common with our work, Luo introduces observational equalities and demonstrates their usefulness in specifying abstract data types.

The definition of specifications in ECC as pairs means that the implementation relationship between specifications and implementations in MLT (i.e. that a specification is a type and its implementations are all the members of the type) does not hold between specifications and implementations in ECC. Nevertheless, implementations of specifications in ECC have a similar definition to modules in MLT: an implementation of a specification $S P$ in ECC is defined as a pair consisting of any member $m$ of the structure type of $S P$, together with a witness that $m$ satisfies $A x(S P)$ (i.e. 
that $A x(S P)(m)$ is inhabited). The set of all implementations of $S P$ is given by the type $\sum m \in \operatorname{Str}(S P) . A x(S P)(m)$; note the similarity of this type to our notion of specification in MLT.

We now compare the specification operators in our specification language with those defined by Luo. Luo does not define any specification operators that are similar in function to our rename or hide operators since the components of specifications in ECC are not named, and there is no mechanism for specifying local components. However, the remaining specification operators in our specification language correspond to specification operators defined by Luo. Luo defines an infix binary specification operator $\otimes$ which is similar in function to our sum operator: for any two specifications $S P_{1}$ and $S P_{2}, S P_{1} \otimes S P_{2}$ specifies the components specified by $S P_{1}$ and $S P_{2}$. The structure type of $S P_{1} \otimes S P_{2}$ is defined as $\operatorname{Str}\left(S P_{1}\right) \times \operatorname{Str}\left(S P_{1}\right)$, using the product type constructor $(x)$. Luo does not need an operator as sophisticated as signature concatenation - which we use to combine signatures in our definition of sum-to combine structure types since there is no formal namespace for components in structure types, and therefore the issue of combining namespaces does not arise.

Luo also defines an operator called Extend which extends a specification by adding some extra components and/or axioms. Extend is similar in function to our enrich operator. For example, the specification $\operatorname{Extend}(S P, S, E)$ is specification $S P$ extended by some extra components given by an extension $S$, which is function of type $S \in \operatorname{Str}(S P) \rightarrow$ Type, and some axioms $E$ over the extended structure type $\left(E \in\left(\sum x \in \operatorname{Str}(S P) . S(x)\right) \rightarrow\right.$ Prop $)$. The extension $S$ can be seen as a structure type that is dependent on the components supplied by $\operatorname{Str}(S P)$; extensions like $S$ play the same role as dependent signatures in our work.

Luo defines the operator Join which is identical in function to our union operator. Given two specifications $S P_{1}$ and $S P_{2}$ with the same structure type- $S$, say-the specification $\operatorname{Join}_{S}\left(S P_{1}, S P_{2}\right)$ has structure type $S$, and its axiomatic part is the conjunction of the axiomatic parts of $S P_{1}$ and $S P_{2}$. Luo also defines two operators Con (called constructor) and $\mathrm{Sel}$ (called selector) which are identical in function to our derive and translate operations, respectively. An application of Con has the form $\operatorname{Con}_{f}(S P)$ for any specification $S P, f \in \operatorname{Str}(S P) \rightarrow S$ and $S$ any structure type. The function $f$ plays the same role as a derive map. An application of $S e l$ has the form $\operatorname{Sel}_{g}(S P)$ for any specification $S P, g \in S \rightarrow \operatorname{Str}(S P)$ and $S$ any structure type. The function $g$ plays the same role as a translate map.

Specifications in ECC are implemented through stepwise refinement just like our specifications in MLT: a specification is refined through a sequence of refinement steps until its axioms strongly suggest an implementation which can then be constructed via a constructive proof. Luo's definition of refinement is very similar to our definition of specification refinement in MLT: 


\section{Definition 10.1 (Specification Refinement in ECC)}

A specification $S P_{1}$ refines to a specification $S P_{2}$ through a refinement map

$$
h \in \operatorname{Str}\left(S P_{2}\right) \rightarrow \operatorname{Str}\left(S P_{1}\right)
$$

(written $S P_{1} \Longrightarrow_{h} S P_{2}$ ) if the following proposition is provable:

$$
\forall x \in \operatorname{Str}\left(S P_{2}\right) \cdot A x\left(S P_{2}\right)(x) \Rightarrow A x\left(S P_{1}\right)(h(x))
$$

The refinement map in the above definition plays the same role as an abstraction map in our definition of refinement in MLT; and a witness to proposition (1) is equivalent to a proof map in our definition of refinement. Like our refinement relation $\sqsubseteq$, the refinement relation $\Longrightarrow$ is transitive. $\Longrightarrow$ is also monotonic with respect to the specification operators defined by Luo.

Luo's work does not address the issue of implementors (as we do in Chapter 9) for implementing structured specifications. However, unlike our work, Luo's work addresses the issue of implementing structured specifications whose individual sub-specifications share common data-types and/or operations. Such "sharing" may prevent a programmer from implementing the sub-specifications of a structured specification independently of each other, but Luo shows how a programmer can overcome this problem by using $\sum$-types to restructure such specifications.

Parameterised specifications in ECC are functions that return specifications as their result; as are parameterised specifications in our work. The parameters of a parameterised specification in ECC (and in our specification language) can be any values in type theory, including types, functions, program modules and specifications. Unlike our work, Luo addresses the interesting issue of implementing parametersised specifications. In particular, Luo gives a definition of refinement for parameterised specifications, together with some laws for refining parameterised specifications.

Finally, we return to the issue of the type SPEC of all specifications in ECC, described earlier in this section. The type SPEC allows Luo to define parameterised specifications-including specification operators, which are a special case of parameterised specifications-more concisely than we can in our specification language. For example, Luo may use $S P E C$ to define parameterised specifications of the form $\lambda x \in S P E C . S P(x)$ (with type $S P E C \rightarrow S P E C$ ) which are parameterised over specifications. In our specification language, we define such parameterised specifications in the form $\lambda x \in U_{1} . S P(x)$ (with type $U_{1} \rightarrow U_{1}$ ); note that we can only give specifications the type $U_{1}$. Consequently, in our specification language, we have to define the body, $S P(x)$, of such parameterised specifications as being well-typed for all types $x \in U_{1}$, and not just specifications, since functions in MLT (and ECC) must be total. This adds to the work of making such parameterised specifications in our specification language; although the additional work is not technically challenging. 
In conclusion, Luo's work is an extremely important contribution to the study of constructing and refining modular specifications in type theory. It is interesting to note that there are many points in common between Luo's work and ours, even though they are set in different type thoeries.

\subsection{A Final Comment}

This thesis has demonstrated that type theory provides an excellent framework for both the specification and development of program modules. We have shown how type theory can be used to give a formal semantics to a module specification language, and an associated implementation language. We have also shown that implementations of modular specifications may be calculated by systematic refinement. 


\section{Appendix A}

\section{A.1 Dot Notation}

The semantics of specifications and modules have been defined to include names for components. The names are intended to allow us to refer to the individual components of modules by using a Pascal style dot notation. In this appendix, we give a formal definition of dot notation on computational elements and modules.

Dot notation is defined using value selector functions on tuples; tuples are nested pairs of the form $\left\langle e_{0},\left\langle e_{2}, \ldots\left\langle e_{n-1}, e_{n}\right\rangle \ldots\right\rangle\right\rangle$. Value selectors return the values of components in tuples via their position in the tuple. For example, the component at position $i$ $(i \in \mathbb{N})$ in a tuple $b \in B$ is referred to as $b_{B} . i$. To calculate the type of any component referred to by dot notation on computational elements and modules, we will also define a form of dot notation that returns the types of components in signatures or specifications. Dot notation on signatures and specifications is defined using type selector functions on tuple types; tuple types are nested $\sum$-types of the form $\sum y_{0} \in$ $A_{0} \ldots \sum y_{n-1} \in A_{n-1} \cdot A_{n}$. Type selectors return the type of any component $b_{B} . i$ in a tuple $b \in B$ where $B$ is a tuple type.

We begin by defining value and type selector functions on tuples. We then define dot notation on computational elements and signatures. Then we define dot notation on modules and specifications.

\section{A.1.1 Selector Functions}

In this section, we define functions that return the values and types of individual components within tuples. Functions that return the values in tuples are called value selectors, and functions that return the types of the values in tuples are called type selectors. Previously, tuples have been assumed to be nested pairs, so that tuple types are nested $\sum$-types. However, selector functions are generalised so that they can be 
applied to non-pair values. A non-pair value is any value whose type is not a $\sum$-type. Non-pair values are thought of as "tuples" containing a single value.

\section{Value Selectors}

Every tuple type has its own value selector function; just as every pair type has its own projection functions. Consider the tuple $b=\left\langle e_{0},\left\langle e_{2}, \ldots\left\langle e_{n-1}, e_{n}\right\rangle \ldots\right\rangle\right\rangle$ that is assumed to have type $B=\sum y_{0} \in A_{0} \ldots \sum y_{n-1} \in A_{n-1} . A_{n}$. An application of value selection to $b$ has the form $b_{B} . i$ for any index $i \in \mathbb{N}$. $b_{B} . i$ is the component at position $i$ in tuple $b$. The value of $b_{B} . i$ is given by:

$$
\begin{aligned}
& b_{B} \cdot i=e_{i}, \quad i \leq n \\
& b_{B} \cdot i=e_{n}, \quad i>n
\end{aligned}
$$

Selector indices are assumed to range from 0 onwards, so that $b_{B} .0$ is the first component in $b$. Note that for any $i>n$, the expression $b_{B} . i$ always equals the final component in $b$; so a selector index is never out of range. Selector functions are defined in terms of the projection functions $f_{s t}$ and snd. Figure A.1 contains a definition of $b_{B} . i$, for all $i \in \mathbb{N}$, in terms of $f s t$ and snd.

For any non $\sum$-type $R$, the value selector function on members of $R$ is defined such that for any $r \in R, r_{R} . i=r$. In other words, a value selector function on members of a non $\sum$-type acts like an identity function, and ignores the selector index.

\section{Type Selectors}

To calculate the type of any component referred to by a value selector, we will define functions, called type selectors, that return the type of a component in a tuple. Consider the tuple type $B=\sum y_{0} \in A_{0} \ldots \sum y_{n-1} \in A_{n-1}$. $A_{n}$. An application of type selection has the form $B_{b} . i$ for any tuple $b \in B$ and index $i \in \mathbb{N}$. $B_{b} . i$ is the type of the component at position $i$ in tuple $b$. The type of each component in any $b \in B$ is given as follows:

$$
\begin{array}{ll}
b_{B} . i \in B_{b} \cdot i=A_{i}\left(y_{0} \backslash b_{B} \cdot 0, \ldots, y_{i-1} \backslash b_{B} \cdot(i-1)\right) & , i \leq n \\
b_{B} . i \in B_{b} . i=A_{n}\left(y_{0} \backslash b_{B} \cdot 0, \ldots, y_{n-1} \backslash b_{B} \cdot(n-1)\right), & i>n
\end{array}
$$

Note that the type of a component $b_{B} . i$ may be dependent on the components $b_{B} \cdot 0, \ldots, b_{B} \cdot(i-1)$ in $b$.

Given any non-tuple type $R$, type selection on any $r \in R$ is defined so that $R_{r} . i=R$ for any index $i \in \mathbb{N}$. Defining type selection functions over all types is mathematically appealing since it yields the following relationship between value and type selectors:

$$
\forall B \in U_{1} . \forall b \in B . \forall i \in \mathbb{N} .\left(b_{B} . i \in B_{b} . i\right)
$$




$$
b_{B} \cdot i= \begin{cases}f s t(b) & , \quad i=0 \\ f s t(\operatorname{snd}(b)) & , i=1 \\ \vdots & , \quad i=n-1 \\ f_{s t}(\operatorname{snd}(\ldots \operatorname{snd}(b) \ldots)) & , \quad i \geq n \\ \operatorname{snd}(\operatorname{snd}(\ldots \operatorname{snd}(b) \ldots)) & , \quad i \geq\end{cases}
$$

Figure A.1: Value Selection on Tuples of Type $B$

\section{A Technical Definition of Value Selectors}

A formal definition of value selection is given by defining a function VS (value select) that takes a type and returns its value selector function. The type of $V S$ is given below:

$$
V S \in \prod B \in U_{1} \cdot \prod b \in B . \prod i \in \mathbb{N} .\left(B_{b} . i\right)
$$

Using $V S$, the expression $b_{B} . i$ is defined as

$$
b_{B} \cdot i \equiv V S(B)(b)(i) .
$$

Function $V S$ is defined in terms of $f s t$ and $s n d$. The definition of $V S$ is presented in a clausal form, and is given in Definition A.1.

\section{Definition A.1 (Value Selectors)}

Given any type $B, b \in B$ and index $i \in \mathbb{N}$ then $b_{B} . i \equiv V S(B)(b)(i) . V S$ is defined inductively over the structure of type $B$ as follows:

$$
\begin{array}{llc}
V S(R)(b)(i) & = & \left(R \text { not a } \sum \text {-type }\right) \\
V S\left(\sum x \in P . Q(x)\right)(b)(i) & = & \text { if } i=0 \text { then } f s t(b) \text { else } V S(Q(f s t(b)))(\operatorname{snd}(b))(i-1)
\end{array}
$$

In the above definition, the expression $V S\left(Q\left(f_{s t}(b)\right)\right)(\operatorname{snd}(b))(i-1)$ is the component at position $(i-1)$ in $\operatorname{snd}(b)$ where $s n d(b) \in Q(f s t(b))$ since $b \in \sum x \in P . Q(x)$.

\section{A Technical Definition of Type Selectors}

A formal definition of type selector functions is given in terms of a function TS (type select) that takes a type and returns its type selector function. The type of $T S$ is given below:

$$
T S \in \prod B \in U_{\mathbf{1}} \cdot \prod b \in B . \prod i \in \mathbb{N} .\left(U_{\mathbf{1}}\right)
$$

Using $T S$, the type $B_{b} . i$ is defined as:

$$
B_{b} . i \equiv T S(B)(b)(i) .
$$

Function $T S$ is defined over type terms and is similar, in style, to the definition of $V S$. The definition of $T S$ is given in Definition A.2. 


\section{Definition A.2 (Type Selectors)}

Given any type $B, b \in B$ and index $i \in \mathbb{N}$ then $B_{b} . i \equiv T S(B)(b)(i) . T S$ is defined inductively over the structure of type $B$ as follows:

$$
\begin{array}{llc}
T S(R)(b)(i) & =R, & \left(R \text { not a } \sum \text {-type }\right) \\
T S\left(\sum x \in P . Q(x)\right)(b)(i) & = & \text { if } i=0 \text { then } A \text { else } T S(Q(f s t(b)))(\operatorname{snd}(b))(i-1)
\end{array}
$$

In the above definition, the expression $T S(Q(f s t(b)))(\operatorname{snd}(b))(i-1)$ is the type of the component at position $(i-1)$ in $\operatorname{snd}(b) \in Q\left(f_{s t}(b)\right)$.

Theorem A.1 Given any $B$ type, $b \in B$ and $i \in \mathbb{N}$ then $b_{B} . i \in B_{b} . i$.

Proof By structural induction on $B$.

\section{A.1.2 Dot Notation on Computational Elements}

In this section, we define a Pascal style dot notation on computational elements and signatures. Given any signature $S$, computational element $m \in S$, and a component name $n \in \mathbb{S}$, the expression $m . n$ is the value of a component named $n$ in $m$. The expression $S_{m} . n$ is the type of $m . n$, i.e. $m . n \in S_{m} . n$. Dot notation on computational elements and signatures may refer to both local and visible components. In the event that $n$ is overloaded in $m$ (and $S$ ), then $m . n$ returns the value of the last (right-most) version of a component named $n$ in $m$.

Dot notation on computational elements is defined using the value selector function $V S$. An expression $m . n$ is defined by first seeking the position- $i$, say-at which $n$ occurs in the domain of $m$, and then using $V S$ to select the component at position $i$ in the value tuple of $m$. Similarly, $S_{m} . n$ is defined by using the type selector TS to calculate the type of the component at position $i$ in the value tuple of $m$. In the event that there is no visible or local component named $n$ in $m$, the expression $m . n$ returns the unit value $t t$; and $S_{m} . n$ returns the unit type $T$.

The definition of dot notation on computational elements and signatures is given in Definition A.3 with an explanation following. The expression

$$
\text { index }(\operatorname{dom}(m))(n)(\text { equalAll })
$$

is the index of the last occurrence of name $n$ in the domain of $m(\operatorname{dom}(m)) ; n$ can be either visible or local. If $n$ is not found in $\operatorname{dom}(m)$ then the index of the last element, $t t$, in the value tuple of $m(v a l(m))$ is returned. $\operatorname{Lsg}(S)$ is the loose signature of $S$, so that $\operatorname{val}(m) \in \operatorname{Lsg}(S)$.

\section{Definition A.3 (Dot notation on computational elements)}

For all signatures $S, m \in S$ and $n \in \mathbb{S}$,

$$
\begin{aligned}
& m . n=V S(\operatorname{Lsg}(S))(\operatorname{val}(m))(\operatorname{index}(\operatorname{dom}(m))(n)(\text { equalAll })) \in S_{m} . n \\
& S_{m} . n=T S(\operatorname{Lsg}(S))(\operatorname{val}(m))(\operatorname{index}(\operatorname{dom}(m))(n)(\text { equalAll })) \in U_{1}
\end{aligned}
$$


The functions index and equalAll are given in Definitions A.4 and A.6, respectively, with an explanation following. The expression index $(l)(n)($ equal) returns the position of the last tagged name-e, say-in domain $l \in \mathbb{D}$, that makes equal $(\mathrm{e}, \mathrm{n})$ true. The expression equalAll $(e, n)$ compares a tagged name, $e$, with an un-tagged name, $n$, and returns true if the two names are equal when the tag on $e$ is ignored. The expression $i \sin (n)(l)($ equal $)$ is true if the name $n$ appears as a tagged name in 1 . Consequently, index $(l)(n)($ equalAll $)$ expresses the position, in $l$, of the last occurrence of component name $n$.

\section{Definition A.4 (index)}

For all $e: l \in \mathbb{D}, n \in \mathbb{S}$ and equal $\in(\mathbb{S}+\mathbb{S}) \rightarrow \mathbb{S} \rightarrow \mathbb{B}$, the function

$$
\text { index } \in \mathbb{S} \rightarrow \mathbb{D} \rightarrow((\mathbb{S}+\mathbb{S}) \rightarrow \mathbb{S} \rightarrow \mathbb{B}) \rightarrow \mathbb{N},
$$

is defined as follows:

$$
\begin{array}{ll}
\text { index }(n)(\text { nil })(\text { equal })= & 0 \\
\text { index }(n)(e: l)(\text { equal })= & \text { if } \operatorname{equal}(e)(n) \wedge \neg(\text { isin }(n)(l)(\text { equal })) \text { then } \\
& 0 \\
& \text { else } \\
& \quad \text { index }(n)(l)(\text { equal })+1
\end{array}
$$

\section{Definition A.5 (isin)}

For all $e: l \in \mathbb{D}, n \in \mathbb{S}$ and equal $\in(\mathbb{S}+\mathbb{S}) \rightarrow \mathbb{S} \rightarrow \mathbb{B}$, isin $\in \mathbb{S} \rightarrow \mathbb{D} \rightarrow \mathbb{B}$ is defined as follows:

$$
\begin{aligned}
& i \sin (n)(\text { nil })(\text { equal })=\text { false } \\
& i \sin (n)(e: l)(\text { equal })=\text { if equal }(e)(n) \text { then true else } i \sin (n)(l)
\end{aligned}
$$

\section{Definition A.6 (equalAll)}

For all $e \in \mathbb{S}+\mathbb{S}$ and $n \in \mathbb{S}$, equalAll $\in(\mathbb{S}+\mathbb{S}) \rightarrow \mathbb{S} \rightarrow \mathbb{B}$ is defined as follows:

$$
\text { equalAll }(e)(n)=((e=\operatorname{inl}(n)) \text { or }(e=\operatorname{inr}(n)))
$$

\section{Restricted Dot Notation on Computational Elements}

In this section, we define a restricted form of dot notation that cannot refer to local components. This restricted form of dot notation will be used later to define dot notation on modules. For any signature $S$, computational element $m \in S$ and name $m \in \mathbb{S}$, the restricted form of dot notation on computational elements has the form $m$. [n]. If $n$ is a visible component name in $m$, or if $n$ is not a component name in $m$, then $m$. $[n]$ is $m . n$. But, if $n$ is a local component name in $m$ then $m$. $[n]$ is the unit value $t t \in T$. The restricted form of dot notation on signatures has the form $S_{m} \cdot[n]$; $S_{m \cdot[n]}$ is the type of $m \cdot[n]$. 
The definition of restricted dot notation is almost identical to the definition of unrestricted dot notation given in the previous section, except that the function equalAll is replaced by the function equalVis given in Definition A.7. An expression equalVis $(e, n)$ is true iff $e$ is a visible name which, when we strip its tag, is equal to the un-tagged name $n$. The definition of restricted dot notation is given in Definition A.8.

\section{Definition A.7 (equalVis)}

For all $e \in \mathbb{S}+\mathbb{S}$ and $n \in \mathbb{S}$, equalVis $\in(\mathbb{S}+\mathbb{S}) \rightarrow \mathbb{S} \rightarrow \mathbb{B}$ is defined as follows:

$$
\text { equalVis }(e)(n)=(e=\operatorname{inl}(n))
$$

\section{Definition A.8 (Restricted Dot extraction)}

For all $S$ sig, $m \in S$ and $n \in \mathbb{S}$,

$$
\begin{aligned}
& m \cdot[n]=\operatorname{VS}(\operatorname{Lsg}(S))(\operatorname{val}(m))(\operatorname{index}(\operatorname{dom}(m))(n)(\text { equalVis })) \in S_{m} \cdot[n] \\
& S_{m} \cdot[n]=T S(\operatorname{Lsg}(S))(\operatorname{val}(m))(\operatorname{index}(\operatorname{dom}(m))(n)(\text { equalVis })) \in U_{1}
\end{aligned}
$$

\section{A.1.3 Dot Notation on Modules and Specifications}

Dot notation on modules allows us to refer to any visible components in modules; and dot notation on specifications allows us to refer to the type of any visible component in a specification. Any dot references to local components in modules and specifications returns the unit value $t t$ and the unit type $T$, respectively. Dot notation on modules and signatures is defined using the restricted form of dot notation on computational elements and signatures.

Definition A.9 (Dot notation on modules and specifications)

For all specification $S P$, modules $m \in S P$ and name $n \in \mathbb{S}$,

$$
\begin{aligned}
& m . n=\operatorname{ce}(m) \cdot[n] \in S P_{m} \cdot n \\
& S P_{m} \cdot n=\operatorname{Sig}(S P)_{c e(m)} \cdot[n] \in U_{1}
\end{aligned}
$$




\section{Bibliography}

[1] S.N. Ahmed and J.M. Morris. Constructing and Refining Modules in a Type Theory. In J.M. Morris, editor, Proceedings of the 4 th Workshop on Refinement, Springer-Verlag (BCS Workshop series), 1991.

[2] R.J.R. Back. Correctness preserving program refinements: proof theory and applications. Mathematical Centre Tracts 131, Mathematisch Centrum, Amsterdam, 1980.

[3] R.C. Backhouse et al. Do-it-yourself type theory. Formal Aspects of Computing, Vol.1, No. 1, January-March 1989.

[4] R.M. Burstall, J.A Goguen. The semantics of CLEAR, a specification language. In Proc. of Advanced Course on Abstract Software Specifications, LNCS 86, Springer, 1981.

[5] R.M. Burstall, J.A Goguen. An Informal Introduction to Specifications Using CLEAR. In N. Gehani, A.D. McGettrick, editors, Software Specification Techniques, Addison-Wesley, 1986.

[6] R.M. Burstall, J. McKinna. Deliverables: an approach to program development in the Calculus of Constructions. In the preliminary Proceedings of the 1st Workshop on Logical Frameworks, 1990.

[7] L. Cardelli. Typeful Programming. Technical Report 45, DEC Systems Research Center, 130 Lytton Ave., Palo Alto, 1989.

[8] R.L. Constable et al. Implementing Mathematics With The NuPRL Proof Development System. Prentice-Hall, Englewood Cliffs, NJ, 1986.

[9] R.L. Constable. Lectures on: Classical Proofs as Programs. In F. L. Bauer et al, editors, Logic and Algebra of Specification, NATO ASI Series, Springer-Verlag, Berlin, 1991.

[10] T. Coquand, G. Huet. The Calculus of Constructions. Information and Computation, 76 (2/3), pages 96-120, 1988. 
[11] E.W. Dijkstra. A Discipline of Programming. Prentice-Hall, Englewood Cliffs, N.J, 1976.

[12] H. Ehrig, H-J. Kreowski, B. Mahr, P. Padawitz. Algebraic implementation of abstract types. Theoretical Computer Science, 20, 1982.

[13] J.S. Fitzgerald, C.B. Jones. Modularizing a Formal Description of a Database System. In D. Bjorner et al, editors, VDM '90 VDM and Z, LNCS 428, Springer, Berlin, 1990.

[14] J.A. Goguen, J.J. Tardo. An Introduction to OBJ: a language for writing and testing formal algebraic program specifications. In N. Gehani, A.D. McGettrick, editors, Software Specification Techniques, Addison-Wesley, 1986.

[15] J.A. Goguen, J.W. Thatcher, E.G. Wagner. Initial algebra semantics of continuous algebras. Journal of the ACM, 24, 1977.

[16] J.V. Guttag. The specification and application to programming of abstract data types. Ph.D. Thesis, University of Toronto, 1975.

[17] J.V. Guttag, J.J. Horning. Report on the LARCH shared language. Science of Computer Programming, 6, pages 103-134, 1986.

[18] R. Hennicker. Observational Implementations. In B. Moniens, R. Cori, editors, Proc. STACS 89, LNCS 349, Springer, Berlin, 1989.

[19] C.A.R. Hoare. Proofs of Correctness of Data Representations. Acta Informatica, 1, pages 271-281, 1972.

[20] W.A. Howard. The formulae-as-types notion of construction. In J. P. Seldin and J. R. Hindley, editors, To H.B. Curry: Essays on Combinatory Logic, Lambda Calculus and Formalism, pages 479-490. Academic Press, London, 1980.

[21] C.B. Jones. Systematic Software Development using VDM. Prentice-Hall, Englewood Cliffs, NJ, 1986.

[22] S. King. $Z$ and the Refinement Calculus. In D. Bjorner et al, editors, VDM ' 90 $V D M$ and Z, LNCS 428, Springer, Berlin, 1990.

[23] Z. Luo, R. Pollack, and P. Taylor. How to Use LEGO: a preliminary user's manual. LFCS Technical Note LFCS-TN-27, Dept. of Computer Science, Edinburgh University, 1989.

[24] Z. Luo. ECC, an extended Calculus of Constructions. In Proc. of the Fourth Annual Symposium on Logic in Computer Science, Asilomar, California, U.S.A, June 1989. 
[25] Z. Luo. A Higher-Order Calculus and Theory Abstraction. Information and Computation, 90, pages 107-137, 1991.

[26] Z. Luo. An Extended Calculus of Constructions. Ph.D. Thesis, University of Edinburgh, 1990.

[27] Z. Luo. Program Specification and Data Refinement in Type Theory. LFCS Technical Notes ECS-LFCS-91-131, Dept. of Computer Science, Edinburgh University, 1991.

[28] P. Martin-Löf. A Theory of Types. Technical report 71-3, University of Stockholm, 1971.

[29] P. Martin-Löf. An Intuitionistic Theory of Types. Technical report, University of Stockholm, 1972.

[30] P. Martin-Löf. Constructive Mathematics and Computer Programming. In Logic, Methodology and Philosophy of Science, 6, 1979, North-Holland, Amsterdam, 1982.

[31] P. Martin-Löf. Intuitionistic Type Theory. Bibliopolis, Naples, 1984.

[32] C.A. Middelburg. VVSL: A Language for Structured VDM Specifications. Formal Aspects of Computing, Vol.1, No. 1, January-March 1989.

[33] R. Milner, M. Tofte and R. Harper. The Definition of Standard ML. MIT Press, 1990.

[34] J. Mitchell, G. Plotkin. Abstract types have existential type. In Proc. of the 12th ACM Symposium on Principles of Programming Languages, New York, 1985.

[35] C. Morgan. The Specification Statement. ACM Transactions on Programming Languages and Systems, Vol.10, No.3, pages 403-419, July 1988.

[36] C. Morgan and P.H.B. Gardiner. Data Refinement by Calculation. Acta Informatica, 27, pages 481-503, 1990.

[37] J.M. Morris. A Theoretical Basis For Stepwise Refinement and the Programming Calculus. Science of Computer Programming, 9, pages 287-306, 1987.

[38] J.M. Morris. The Laws of Data Refinement. Acta Informatica, 26, pages 287-308, 1989.

[39] J.M. Morris and S.N. Ahmed. Designing and Refining Specifications with Modules. In J. Woodcock, editor, Proceedings of the 3rd Workshop on Refinement, Springer-Verlag (BCS Workshop series), 1990. 
[40] P. Mosses. Action Semantics. Cambridge Tracts in Theoretical Computer Science, 26, Cambridge University Press, 1992.

[41] C. Murthy. Extracting Constructive Content from Classical Proofs. PhD thesis, Cornell University, Department of Computer Science, 1990.

[42] B. Nordström. Terminating General Recursion. BIT, 28 (3), pages 605-619, October 1988.

[43] B. Nordström and K. Petersson. The Semantics of Module Specifications in Martin-Löf's Type Theory. Report 36, Programming methodology group, Department of computer science, University of Gothenburg, 1985.

[44] B. Nordström, K. Petersson and J.M. Smith. Programming in Martin-Löf's Type Theory. Oxford Clarenden Press, Oxford, 1990.

[45] L. C. Paulson. Constructing Recursion Operators in Intuitionistic Type Theory. Journal of Symbolic Computation, 2, pages 325-355, 1986.

[46] A. Salvesen and J.M. Smith. The Strength of the Subset Type in Martin-Löf's Type Theory. Report 45, Programming methodology group, Department of computer science, University of Gothenburg, 1985.

[47] A. Sampaio and S. Meira. Modular Extensions to Z. In D. Bjorner et al, editors, $V D M$ '90 VDM and Z, LNCS 428, Springer, Berlin, 1990.

[48] D. Sannella. Formal Program Development in Extended ML for the Working Programmer. In J. Woodcock, editor, Proceedings of the 3rd Workshop on Refinement, Springer-Verlag (BCS Workshop series), 1990.

[49] D.T. Sannella and A. Tarlecki. Program specification and development in Standard ML. In Proc. 12th Annual ACM Symposium on Principles of Programming Languages, 1985.

[50] D.T. Sannella and A. Tarlecki. Towards formal development of programs from algebraic specifications: implementations revisited. In H. Ehrig et al, editors, TAPSOFT '87, LNCS 249, Springer, Berlin, 1987.

[51] D. Sannella and M. Wirsing. A Kernel Language for Algebraic Specifications and Implementations. Technical Report CSR-155-83, Dept. of Computer Science, Edinburgh University, 1983.

[52] J.M. Spivey. Understanding Z: A Specification Language and its Formal Semantics. Cambridge Tracts in Theoretical Computer Science, 3, Cambridge University Press, 1988. 
[53] J.E. Stoy. Denotational Semantics: The Scott-Strachey Approach to Programming Language Theory. Cambridge, Massachusetts, MIT Press, 1977.

[54] M. Wand. Final algebra semantics and data type extensions. Journal of Computer System Science, 19, 1981.

[55] M. Wirsing. Structured Algebraic Specifications: A Kernel Language. Theoretical Computer Science, 42, pages 123-249, 1986.

[56] M. Wirsing and M. Broy. A Modular Framework for Specification and Implementation. Invited paper in J. Diaz, F. Orejas, editors, TAPSOFT 89, LNCS 351, Springer, Berlin, 1989.

[57] N. Wirth. Program development by stepwise refinement. Communications of the ACM, 14, pages 221-227, April 1971. 